\title{
Betriebswirtschaftliche Analyse der Möglichkeiten und Grenzen für den Vertragsnaturschutz im Wald
}

Dissertation zur Erlangung des Doktorgrades (Dr. forest.) der

Fakultät für Forstwissenschaften und Waldökologie der

Georg-August-Universität Göttingen

vorgelegt von

Georg Moritz Freiherr von Blomberg

geboren am 13.03.1989 in Gehrden 
1. Gutachter: Prof. Dr. Bernhard Möhring

2. Gutachter: Prof. Dr. Matthias Dieter

Tag der mündlichen Prüfung: 06.05.2021 


\section{Danksagung}

Diese Arbeit entstand im Rahmen des Forschungsprojekts „Vertragsnaturschutz im Wald - AnaIyse der waldökologischen, ökonomischen und rechtlichen Optionen (WaVerNa - Projekt)“, einem vom Bundesministerium für Ernährung und Landwirtschaft (BMEL) geförderten Verbundvorhaben der Nordwestdeutschen Forstlichen Versuchsanstalt, der Universität Hamburg, des Johann Heinrich von Thünen-Instituts und der Georg-August-Universität Göttingen. Dem BMEL als Mittelgeber und der Fachagentur Nachwachsende Rohstoffe e.V. als Projektträger gilt besonderer Dank für die Ermöglichung des Forschungsvorhabens. Für die intensive fachliche Auseinandersetzung zum Vertragsnaturschutz im Wald, die so manchen neuen Blickwinkel ermöglicht hat, möchte ich allen Beteiligten der verschiedenen Forschungsinstitutionen danken.

Besonderer Dank gilt meinem Doktorvater Prof. Dr. Bernhard Möhring, der in großer Geduld den Entstehungsprozess dieser Arbeit stets motivierend und fachlich bereichernd begleitet hat. Ich danke Ihnen für die Möglichkeit der wissenschaftlichen Weiterqualifikation und die unverbrüchliche Treue. Danken möchte ich auch Prof. Dr. Matthias Dieter, der nicht nur im WaVerNa-Projekt stets entscheidende Gedankenanstöße geliefert hat, sondern auch die Zweitbegutachtung meiner Arbeit übernommen hat. Ich danke Ihnen für die angenehme Zusammenarbeit und Ihre wertvollen Hinweise zur Weiterentwicklung meiner Arbeit.

Prof. Dr. Max Krott gilt Dank als Drittprüfer der Arbeit. In äußerst angenehmen Gesprächen konnte so auch mein forstpolitisch-externe Blick auf den Vertragsnaturschutz im Wald gelenkt werden. Ich danke Ihnen für Ihre Unterstützung und die wichtigen Anstöße.

Große Teile dieser Arbeit entstanden während meiner Tätigkeit als wissenschaftlicher Mitarbeiter der Abteilung Forstökonomie der Georg-August-Universität Göttingen. Meinen langjährigen Kollegen danke ich für die inhaltlich und persönlich wichtigen Flurgespräche und den engen Zusammenhalt in der gesamten Zeit der Promotion. Ich freue mich auf das Wiedersehen mit jedem Einzelnen von Euch.

Ich danke meinen Kollegen und Partnern der BB Göttingen GmbH, stellvertretend Dr. Frederik Volckens, die mich immer unterstützt und mir die nötigen Freiräume geschaffen haben.

Abschließend geht der wichtigste Dank an meine Familie und meine liebe Frau Anja, die durch alle Untiefen an meiner Seite gestanden und mich stets motiviert haben, das Ziel im Auge zu behalten und diese Arbeit zu einem erfolgreichen Abschluss zu bringen. Ohne Euch und vor allem ohne Dich läge diese Arbeit heute nicht vor. 


\section{Kurzfassung}

Vertragsnaturschutz im Wald stellt trotz vielfacher wissenschaftlicher und politischer Anstrengungen im Gegensatz zum Vertragsnaturschutz in der Landwirtschaft nur eine Randerscheinung dar. Neben geringen Umsetzungen europäischer Fördermöglichkeiten in nationale Programme bereiten die wenigen Ansätze in der Praxis oftmals Probleme hinsichtlich der Höhe des Entgelts, der Kontinuität der Finanzierung sowie der rechtlichen Regelungen. Das Ziel der vorliegenden Arbeit ist die Analyse des Status quo von Vertragsnaturschutz im Wald mit seinen Hemmnissen und Problemen, die Ableitung erfolgsrelevanter Faktoren aus der Landwirtschaft sowie die Weiterentwicklung von Bewertungskonzepten zur Verbesserung der Ermittlung betrieblicher Belastungen. Anhand einer Befragung von Forstbetrieben wird untersucht, welche Hemmnisse sich bei bisherigen Vertragsnaturschutzmaßnahmen identifizieren lassen und welchen Einfluss dies auf identifizierte Erfolgsfaktoren für eine erfolgreiche Etablierung neuer Maßnahmen hat. Besonderes Augenmerk liegt dabei auf der Frage, welche Erkenntnisse sich aus den erfolgreichen Maßnahmen in der Landwirtschaft für den Forstsektor ableiten lassen. Deutlich wird, dass insbesondere die mangelnde Langfristigkeit der Finanzierung von Vertragsnaturschutzprogrammen sowie die Höhe des Maßnahmenentgelts in Verbindung mit mangelnder Akzeptanz bei allen Akteuren die größten Hemmnisse darstellen. Verbesserungsmöglichkeiten könnten sich aus der Loslösung der finanziellen Mittel aus dem Haushaltsvorbehalt und einer stärkeren Ergebnisorientierung von Maßnahmen zur Stärkung der Waldbesitzer als verantwortlich Handelnde ergeben. Zur Bestimmung der Mindestentgelthöhe von Vertragsnaturschutzmaßnahmen bedarf es praktisch anwendbarer und aktueller Bewertungskonzepte. Zwei dieser Konzepte werden in dieser Arbeit vorgestellt, einer Aktualisierung und Erweiterung unterzogen und in einem konkreten Fallbeispiel angewendet. Die ökonomische Analyse der Vertragsnaturschutzmaßnahmen wird ergänzt um begleitende Faktoren, wie besondere betriebliche Belastungen, die aus der Umsetzung resultieren und steigende Risiken bspw. in der Verkehrssicherung. Bei allen Vertragsnaturschutzmaßnahmen muss neben dem Entgelt und der rechtlichen Ausgestaltung auch ein besonderes Augenmerk auf die Integrierbarkeit in betriebliche Abläufe gelegt werden, um entstehende Risiken zu minimieren.

Schlüsselwörter: Vertragsnaturschutz, Nutzungseinschränkungen, Natura 2000, Alt- und Habitatbaumerhalt, leitfadengestütztes Experteninterview, Agrarumweltmaßnahmen, ELER, GAP 


\section{Abstract}

Despite many scientific and political efforts, contractual nature conservation in forests is a lot less common compared to contractual nature conservation in agriculture. Implementation of European funding into national programs is limited or not feasible in practice regarding the level of compensation, continuity of funding and legal regulations. The aim of the present study is to analyze the status quo of contractual nature conservation in forests with its obstacles and problems, to derive factors of success from agriculture and to further improve evaluation methods to determine the operational burden. By means of a survey of forest enterprises, obstacles in existing contractual nature conservation measures will be identified and their influence on the successful establishment of new measures will be investigated. Particular attention is paid to the possibility to derive successful measures from agricultural and transfer them to the forestry sector. It becomes apparent, that the lack of long-term financing of contractual nature conservation programs and the amount of payments, together with a lack of stakeholder acceptance, are the greatest obstacles. Possible improvement could result from the release of financial resources from the budget reservation and a stronger result-orientation of measures to promote forest owners as responsible actors. Practically applicable and up-to-date evaluation concepts are needed to determine minimum payment/compensation for contractual nature conservation measures. In this thesis, two of these concepts are presented, updated, extended and applied in a concrete case study. The economic analysis of contractual nature conservation measures is supplemented by accompanying factors such as specific operational burdens resulting from implementation and increasing risks, e.g. in traffic safety.

Keywords: Contract nature conservation, restrictions on use, Natura 2000, preservation of habitat trees, guideline-based expert interview, agri-environmental measures, EAFRD, GAP 


\section{Inhaltsverzeichnis}

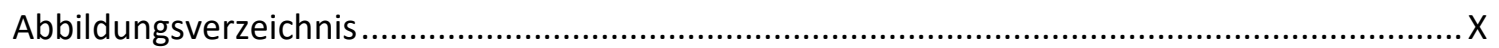

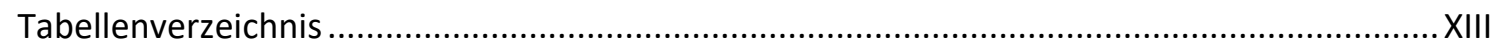

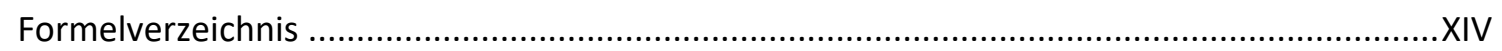

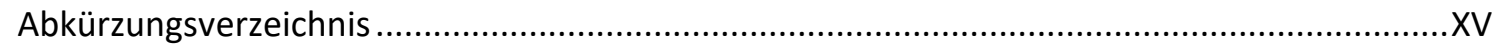

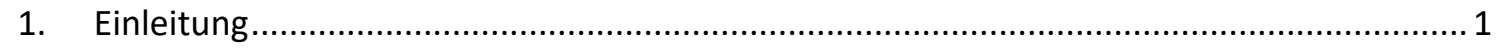

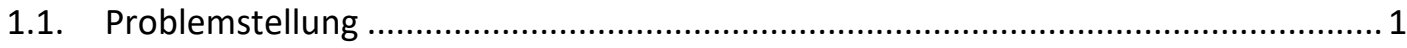

1.2. Einordnung Wald - Naturschutz - Geld ........................................................... 2

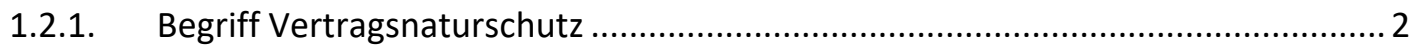

1.2.2. Ausgleichs- und Ersatzmaßnahmen ................................................................. 3

1.3. Ziel und Aufbau der Arbeit ................................................................................. 5

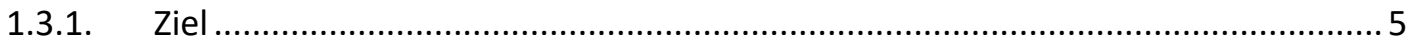

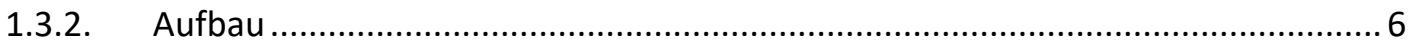

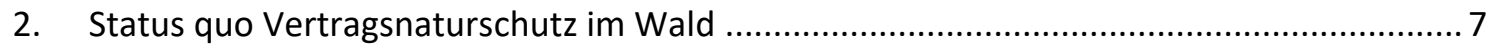

2.1. Rahmenbedingungen für den Vertragsnaturschutz im Wald ................................... 7

2.1.1. Politische Willenserklärungen und tatsächliche Handlungen ................................ 7

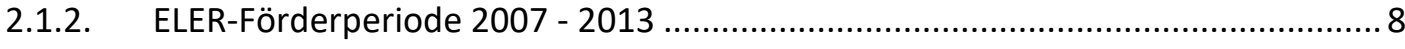

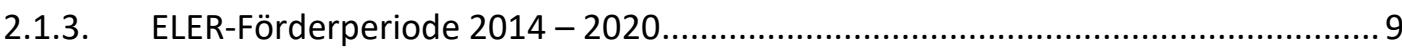

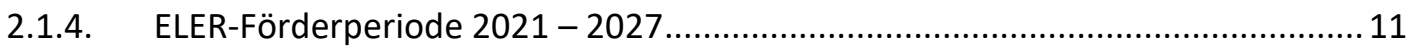

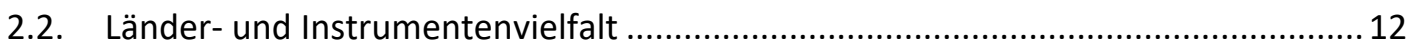

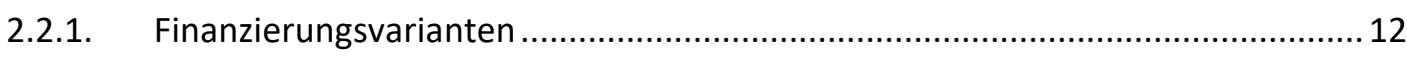

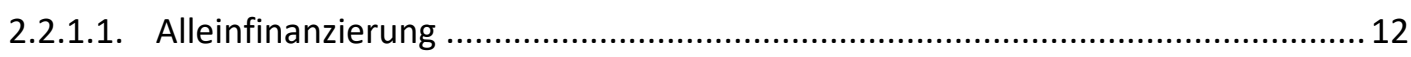

2.2.1.2. Zweistufige Ko-Finanzierung Bund-Land ........................................................ 13

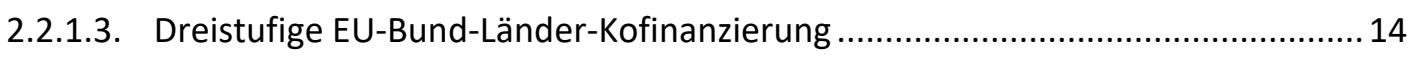

2.2.1.4. Zweistufige EU-Länder-Kofinanzierung ......................................................... 15

2.3. Stand der Umsetzung von Vertragsnaturschutz im Wald in Deutschland ................ 15

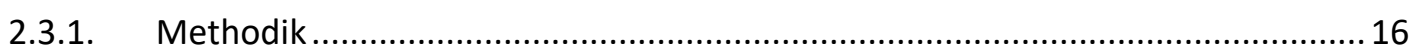

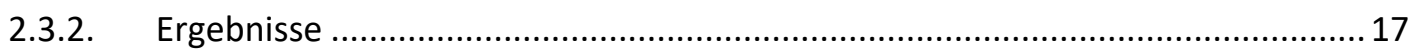

2.4. Potenziale \& Hemmnisse sowie Identifizierung von Erfolgsfaktoren ...................... 19

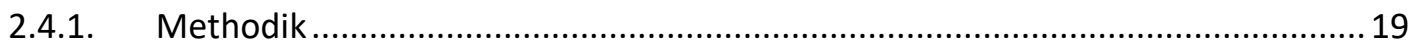

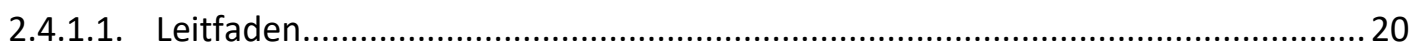

2.4.1.2. Auswertung der Experteninterviews/Fallstudien ............................................. 21

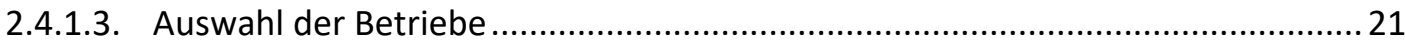

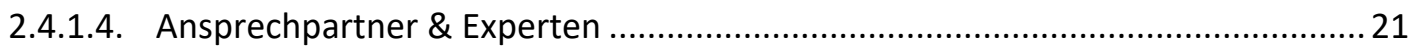

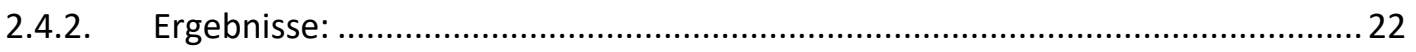




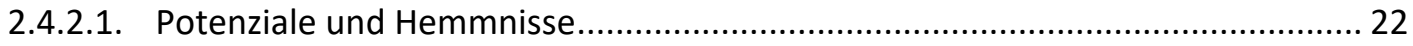

2.4.2.2. Erfolgsfaktoren ................................................................................ 24

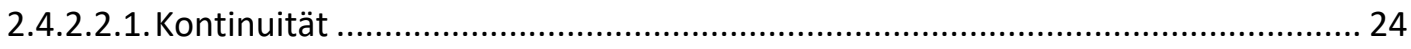

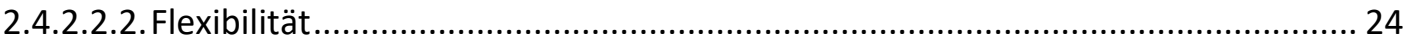

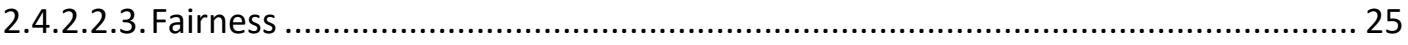

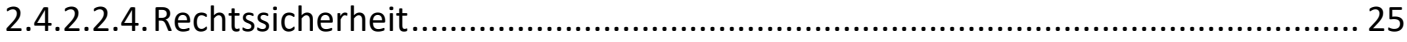

2.4.3. Handlungsempfehlungen \& Lösungsansätze .......................................... 26

2.5. Auswirkungen von Naturschutzmaßnahmen auf Verwaltungs- \& Kontrollkosten ... 27

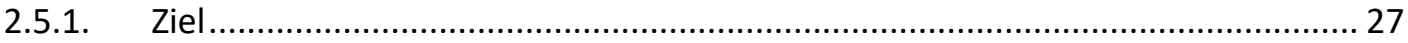

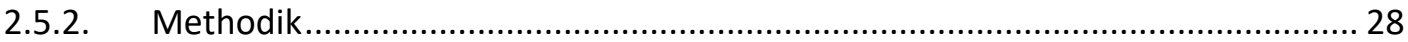

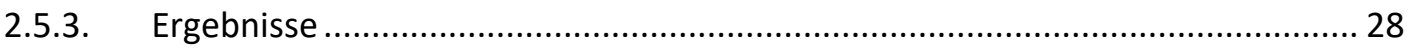

2.5.3.1. Zeiterfassungssysteme in den Fallbeispielsbetrieben .................................. 28

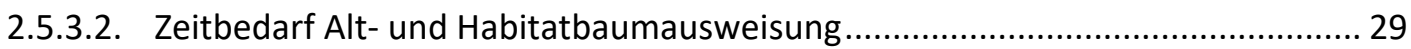

2.6. Vertragsnaturschutz in der Landwirtschaft .................................................. 31

2.6.1. Rechtliche Grundlage \& Finanzielle Ausstattung ........................................ 31

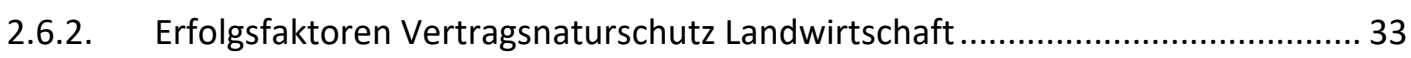

3. Befragung von privaten Forstbetrieben zum Vertragsnaturschutz im Wald .....................36

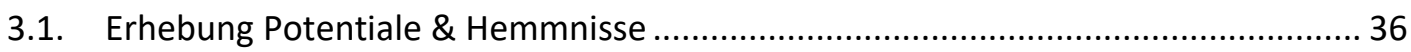

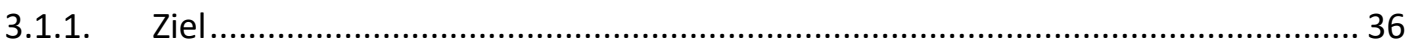

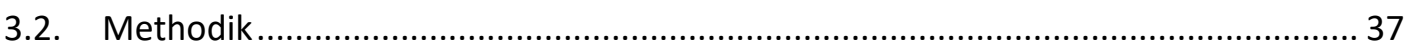

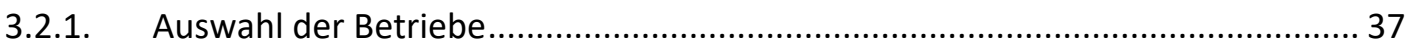

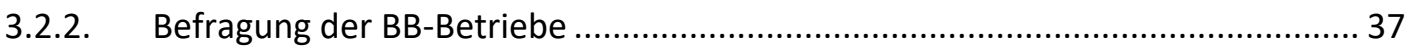

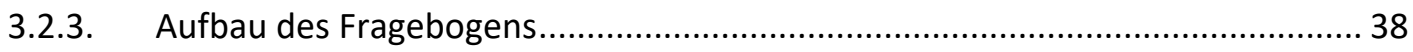

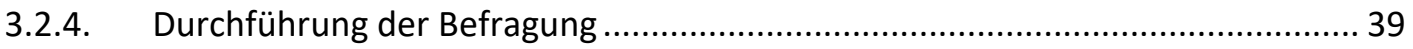

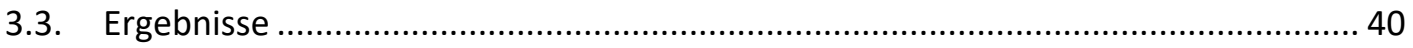

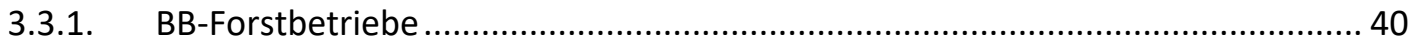

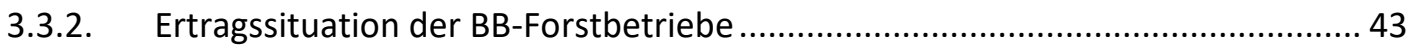

3.3.2.1. Ertragssituation Landwirtschaft .......................................................... 47

3.3.3. Zielsysteme Waldeigentümer .............................................................. 49

3.3.4. Beurteilung Vertragsnaturschutz in der Land- und Forstwirtschaft .................. 54

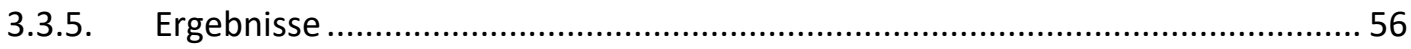

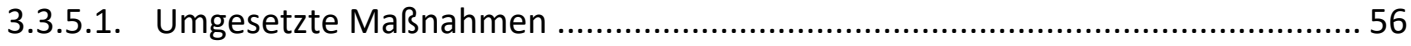

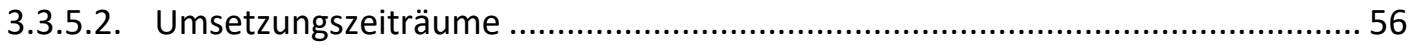

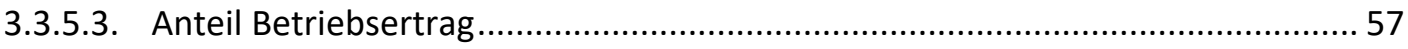

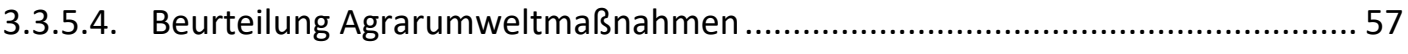

3.3.6. Gründe für Nichtteilnahme an Vertragsnaturschutz im Wald ........................ 57 
3.3.7. Betriebliche Bedeutung des Entgelts für Vertragsnaturschutz im Wald ..............58

3.3.8. Umsetzungsprobleme des Vertragsnaturschutzes im Wald ................................ 58

3.3.9. Stärken von Vertragsnaturschutz im Wald ....................................................... 59

3.3.10. Analyse der Erfolgsfaktoren und Möglichkeiten der Optimierung .......................60

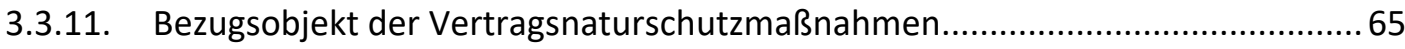

3.3.12. Best-Practice-Beispiele im Wald \& Lösungen aus betrieblicher Sicht................... 66

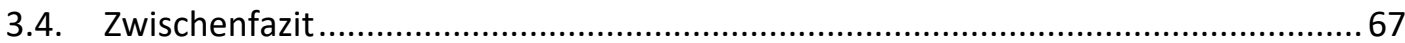

4. Aktualisierung und Weiterentwicklung von forstlichen Bewertungskonzepten .................68

4.1. Ökonomische Auswirkungen durch Bewirtschaftungsänderungen ...........................68

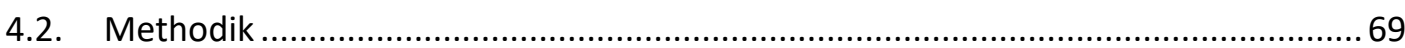

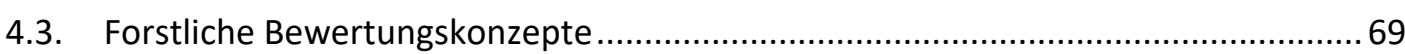

4.3.1. Aktualisierung des Bewertungskonzepts für forstliche Nutzungsbeschränkun-

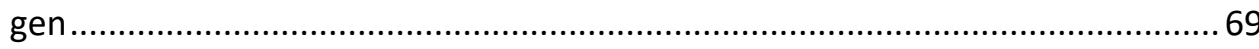

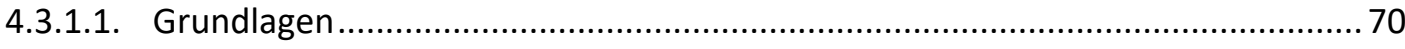

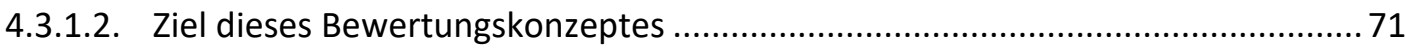

4.3.1.3. Methodisches Konzept des Bewertungsverfahrens........................................... 72

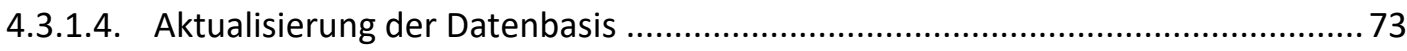

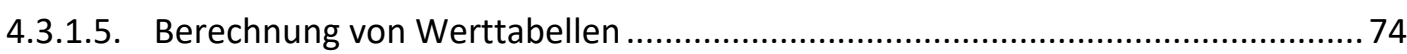

4.3.1.6. Anwendung auf Änderungen der forstlichen Bewirtschaftung ............................76

4.3.1.6.1.Vorübergehender Verzicht auf forstliche Produktion......................................... 76

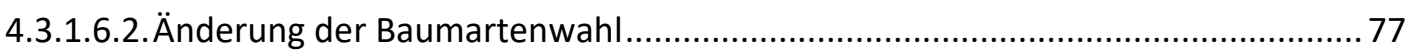

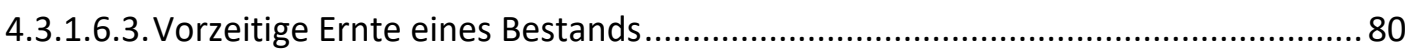

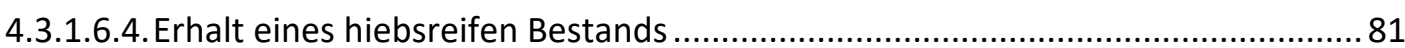

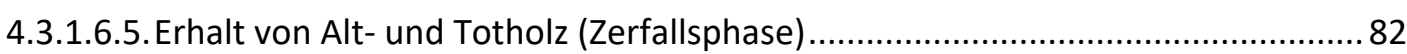

4.3.1.7. Änderungen der Datengrundlagen zwischen 2006 und 2015 .............................. 84

4.3.2. Vergleich von Bewertungstools für forstliche Nutzungsbeschränkungen ............ 86

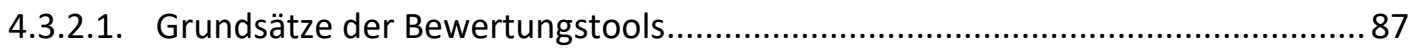

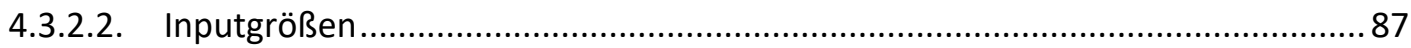

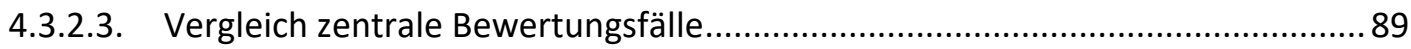

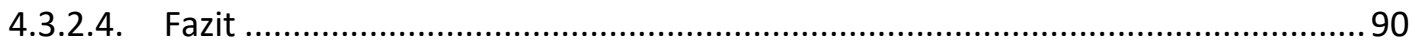

4.3.3. Aktualisierung Bewertungskonzept für Einzelbäume .........................................90

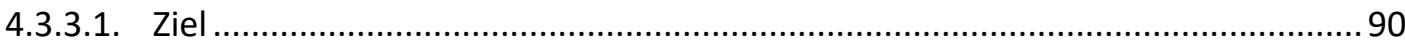

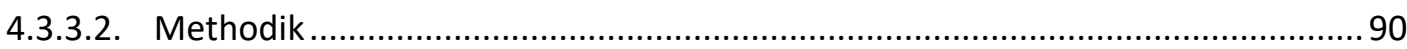

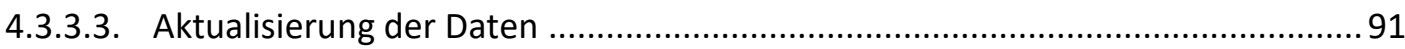

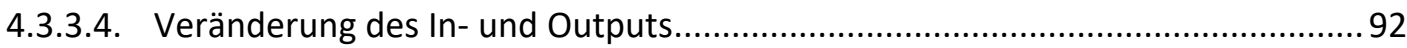

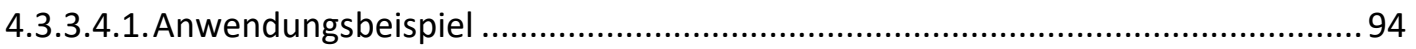


4.3.4. Zusammenfassung Einzelbaumbewertung .................................................... 97

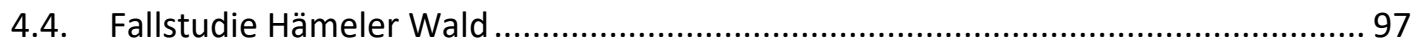

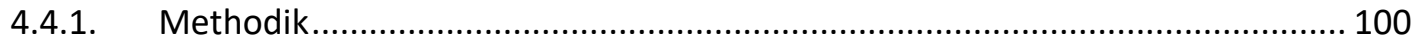

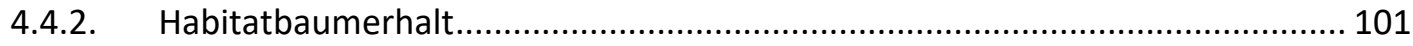

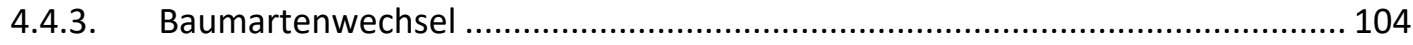

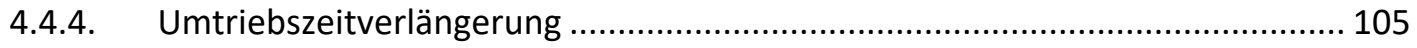

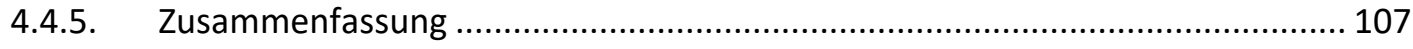

4.5. Auswirkungen verschiedener Verteilungskonzepte von Habitatbäumen .............. 108

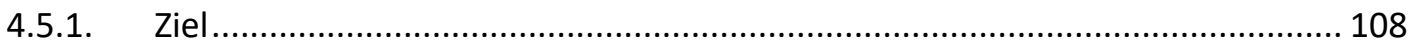

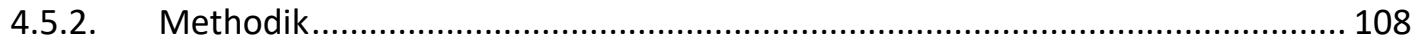

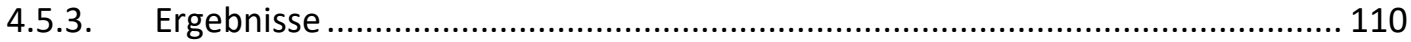

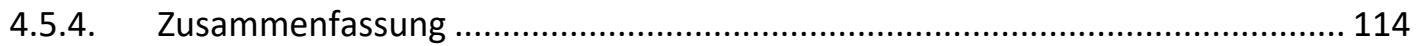

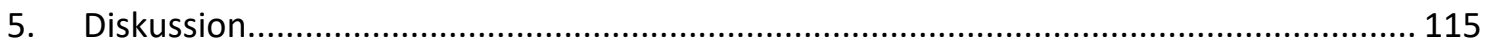

5.1. Diskussion der Befragung von Forstbetrieben .................................................. 115

5.2. Diskussion der ökonomischen Bewertungskonzepte ........................................ 120

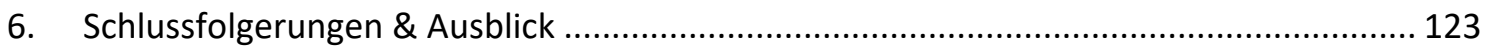

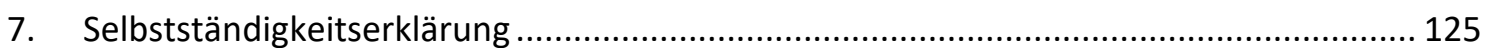

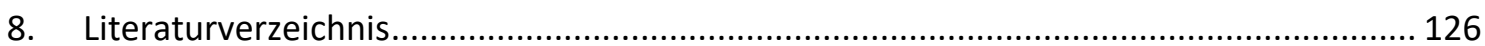

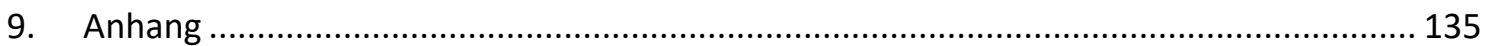

9.1.1. Fragebogen WaVerNa Fallbeispielsanalysen .................................................. 135

9.1.2. Ökologischer Bewertungskatalog Habitatbäume .......................................... 142

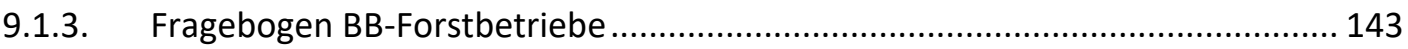

9.1.4. Ergebnisse Bewertungskonzept für Einzelbäume........................................... 147

9.1.5. Eingangsdaten der verglichenen Kalkulationstools für forstliche Nutzungsbe-

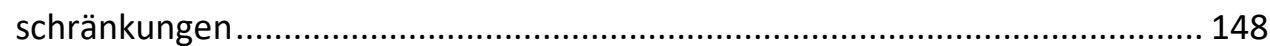




\section{Abbildungsverzeichnis}

Abbildung 1: Grafische Darstellung aktuelles Prämiensystem der 1. Säule der GAP in Deutschland, inkl. Betriebsgrößenberücksichtigung, Quelle: Volckens 2014 ..... 9

Abbildung 2: GAP-Mittel für Deutschland 2014 bis 2020, Quelle: Umweltbundesamt 2018 auf Grundlage BMEL 2018.

Abbildung 3: Finanzierungsvarianten für Waldnaturschutz gegen Entgelt, Quelle: Selzer 2018 .

Abbildung 4: Forstbetriebe der Onlinebefragung mit und ohne Umsetzungserfahrungen von Waldnaturschutz gegen Entgelt, Quelle: Kownatzki et al. 2017

Abbildung 5: Forstbetriebe der Onlineumfrage mit und ohne Umsetzungserfahrungen nach Bundesländern, Quelle: Kownatzki et al. 2017

Abbildung 6: Umgesetzte Waldnaturschutzmaßnahmen gegen Entgelt nach Schutzgebietskategorien der Onlineumfrage, NWE = Natürliche Waldentwicklung, Quelle: Kownatzki et al. 2017

Abbildung 7: Realisierte und beabsichtigte Waldnaturschutzmaßnahmen gegen Entgelt (Mehrfachnennungen möglich, N=195), Quelle: Kownatzki et al. 2017

Abbildung 8: Realisierte und beabsichtigte jährliche Maßnahmenfläche

(Mehrfachnennungen möglich, N=195), Quelle: Kownatzki et al. 2017 19

Abbildung 9: Schematische Darstellung für Lösungsansätze innerhalb der identifizierten Erfolgsfaktoren, Quelle: Franz et al. 2018a 26

Abbildung 10: Entwicklung der GAP Zahlungen in Mrd. € von 1980 - 2020. Quelle: Europäische Kommission, GD Landwirtschaft und ländliche Entwicklung 31

Abbildung 11: Grafische Darstellung der ELER-Komponenten 2014 - 2020 inkl. finanzielle Ausstattung, Quelle: Deutsche Vernetzungsstelle Ländliche Räume. 32

Abbildung 12: Lage der befragten BB-Forstbetriebe nach Bundesländern, $n=40$................... 40

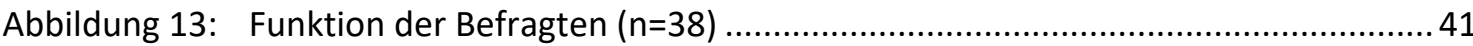

Abbildung 14: Durchschnittliche Betriebsfläche nach Funktion $(n=40)$ 41

Abbildung 15: Größenverteilung Forstbetriebsfläche alle Betriebe $(n=40)$ \& landwirtschaftliche Fläche nur Gemischtbetriebe $(n=16)$.

Abbildung 16: Baumartenanteile in den Baumartengruppen Laubholz $(n=22)$, Fichte $(n=11)$ und Kiefer $(n=7)$

Abbildung 17: Zusammenhang von Fichtenanteil an der Holzbodenfläche und Einschlag sowie Zusammenhang Fichtenanteil an der Holzbodenfläche und Fichtenanteil am Einschlag, $n=38$

Abbildung 18: Zusammenhang von Einschlagsanteil der Baumartengruppe Fichte und erzieltem Deckungsbeitrag I (erntekostenfreier Holzerlös), $n=38$ .46

Abbildung 19: Zusammenhang von Einschlagsanteil der Baumartengruppen Buche und Kiefer und erzieltem Deckungsbeitrag I (erntekostenfreier Holzerlös), $n=38 \ldots 47$

Abbildung 20: Entwicklung der Naturalerträge Landwirtschaft Deutschland West WJ 83/84 bis WJ 17/18, Quelle: BB Göttingen GmbH 
Abbildung 21: Entwicklung landwirtschaftlicher Betriebsertrag, Betriebsaufwand und Grundrente, Deutschland West WJ 83/84 bis 17/18, Quelle: BB Göttingen $\mathrm{GmbH}$..

Abbildung 22: Zielsystem Betriebszweig Forstwirtschaft BB-Forstbetriebe $(n=40)$, zweite Darstellung nur Mittelwert mit Standardabweichung. 50

Abbildung 23: Zielsystem Betriebszweig Landwirtschaft BB-Gemischtbetriebe $(n=18)$. 51

Abbildung 24: Alternative Darstellung Vergleich der land- und forstwirtschaftlichen Ziele der befragten BB-Betriebe, Mittelwerte der Kategorien inkl.

Standardabweichung.

Abbildung 25: Vergleich der land- und forstwirtschaftlichen Ziele der befragten BBBetriebe, Mittelwerte der Kategorien

Abbildung 26: Zielsystem Teilnahme an Naturschutzmaßnahmen gegen Entgelt im Wald BB-Forstbetriebe $(n=25)$.

Abbildung 27: Zielsystem Teilnahme an Vertragsnaturschutzmaßnahmen Landwirtschaft BB-Gemischtbetriebe $(n=17)$.

Abbildung 28: Vergleich der Ziele der landwirtschaftlichen und der forstwirtschaftlichen Betriebe für die Teilnahme an Vertragsnaturschutzmaßnahmen, Mittelwerte der Kategorien.

Abbildung 29: Beurteilung Vertragsnaturschutz in der Land- und Forstwirtschaft, Mittelwerte inkl. Standardabweichung BB-Betriebe mit umgesetzten Maßnahmen ( $n=19)$.

Abbildung 30: Bevorzugte Gestaltung von Vertragsnaturschutzmaßnahmen aus Sicht der BB-Betriebe, $n=27$. 65

Abbildung 31: Aufschub/vorübergehender Verzicht auf forstliche Produktion. 77

Abbildung 32: Änderung der Baumartenwahl (langer Betrachtungszeitraum). 78

Abbildung 33: Änderung der Baumartenwahl (Verrechnung auf 30-jährigen Ausgleichszeitraum) 80

Abbildung 34: Vorzeitige Ernte eines Bestands...... 81

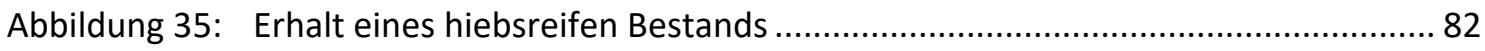

Abbildung 36: Erhalt von Habitatbäumen 83

Abbildung 37: Veränderung der Holzproduktionswerte der fünf Hauptbaumarten von 2006 zu 2015 ohne Berücksichtigung der Kulturkosten.

Abbildung 38: Veränderung der Holzproduktionswerte der fünf Hauptbaumarten von 2006 zu 2015 unter Berücksichtigung der Kulturkosten

Abbildung 39: Vergleich Holzerlös Buche Wertklasse 2, bzw. Wertziffer 4 in $€ / E f m$ in Abhängigkeit des BHD für die drei Kalkulationstools

Abbildung 40: Abtriebswert in Abhängigkeit des BHD inkl. Extrapolation hoher BHD-Stufen bei Douglasie 2014

Abbildung 41: Veränderung der Einzelbaumabtriebswerte in Abhängigkeit vom BHD der Laubbaumarten, 2008 (gestrichelt) zu 2014 (durchgezogen). Wertklasse 2 .... 93

Abbildung 42: Veränderung der Einzelbaumabtriebswerte in Abhängigkeit vom BHD der Nadelbaumarten, 2008 (gestrichelt) zu 2014 (durchgezogen). Wertklasse 2... 93 
Abbildung 43: Vergleich der Abtriebswerte in €/Baum 2010 zu 2018 für die Hauptbaumarten Wkl. 2. Werte auf der schwarz gestrichelten Winkelhalbierenden bedeuten keine Veränderung, Werte oberhalb eine Zunahme, Werte unterhalb eine Abnahme der Abtriebswerte. 94

Abbildung 44: Vergleich der Barwerte Baumart Buche nach Wertklassen in Abhängigkeit vom BHD, sonstige Eingangsdaten unverändert, die Sprünge in den Barwertverläufen resultieren aus Sprüngen in den Einzelbaumabtriebswerten. 96

Abbildung 45: Barwerte der Einzelbäume Buche, Wkl. 2 in Abhängigkeit des unterstellten Wertverlusts über die Laufzeit, sonstige Eingangsdaten unverändert 96

Abbildung 46: Fallstudie Hämeler Wald mit Lebensraumtypflächen, Erhaltungszuständen, Probeflächen und Baumartenverteilung .... 98

Abbildung 47: Stammverteilungsplan Probefläche 1 Hämeler Wald Abt. 1, Punktgröße in Abhängigkeit des Baumdurchmessers, Farbe in Abhängigkeit der Baumart .. 101

Abbildung 48: Stammverteilungsplan Probefläche 1 Hämeler Wald Abt. 1, Gefahrenbereiche der ausgewählten Habitatbäume als farbig-transparente Kreise, $r=$ Baumhöhe 103

Abbildung 49: Stammverteilungsplan Probefläche 2 Hämeler Wald Abt. 2, Punktgröße in Abhängigkeit des Baumdurchmessers, Farbe in Abhängigkeit der Baumart .. 104

Abbildung 50: Stammverteilungsplan Probefläche 3 Hämeler Wald Abt. 3, Punktgröße in Abhängigkeit des Baumdurchmessers, Farbe in Abhängigkeit der Baumart .. 106

Abbildung 51: Lage der untersuchten Flächen im Hochsauerlandkreis. Grün: Lage der untersuchten Flächen, rot: Gebiete der Landschaftspläne. Quelle: Putensen 2018

Abbildung 52: Zusammenhang zwischen BHD und Summe der ökologischen Wertpunkte pro Baum, Quelle: Putensen 2018.

Abbildung 53: Verhältnis von ökologischen Wertpunkten und Gesamtverlust der Einzelbäume, n=161, Quelle: Putensen 2018

Abbildung 54: Veranschaulichung der potenziellen Gefährdungsbereiche an einer Beispielfläche. Szenario Klumpung: Bereich einer Baumlänge (rot): 29 \%, doppelter Baumlänge (lila): 84 \% der Bestandesfläche. Szenario Gleichmäßig: Bereich einer Baumlänge (rot): $144 \%$, doppelter Baumlänge (lila): $368 \%$, Quelle: Putensen 2018

Abbildung 55: Lorenzkurven des relativen Anteils am Gesamtverlust bei Sortierung der 161 aufgenommenen Bäume nach dem ökonomischen Wert und den ökologischen Punkten. Quelle: Putensen 2018 


\section{Tabellenverzeichnis}

Tabelle 1: $\quad$ Potenziale und Hemmnisse von Naturschutzmaßnahmen gegen Entgelt im Wald aus Sicht der befragten Fallbeispielsbetriebe des WaVerNa-Projekts inklusive Handlungsempfehlungen, Zusammenfassung, Quelle: eigene Darstellung

Tabelle 2: Zeitbedarf Umsetzung Naturschutzmaßnahme Alt- und Habitatbäume, Quelle: Fallbeispielsbetriebe WaVerNa-Projekt.

Tabelle 3: $\quad$ Übersicht der Förderhöchstsätze aus Anhang II der Verordnung (EU) 1305/2013, zusammengefasst und abgeändert

Tabelle 4: $\quad$ Naturaldaten befragte BB-Forstbetriebe Wirtschaftsjahr 2017 ( $n=38$, die Abweichung zu der Gesamtzahl von 40 Betrieben resultiert aus zwei Betrieben, die in 2017 nicht am Forstvergleich teilgenommen haben), getrennt nach Baumartengruppen sowie nach Obere 25\%, Durchschnitt aller Betriebe und Untere 25\%, Quelle: BB-Forstbetriebsvergleich 2017

Tabelle 5: $\quad$ Ertrags- und Aufwandsstruktur befragte BB-Forstbetriebe Wirtschaftsjahr 2017 in $€ /$ ha $(n=38)$, getrennt nach Baumartengruppen sowie nach Obere 25\%, Durchschnitt aller Betriebe und Untere 25\%, Quelle: BB-

Forstbetriebsvergleich 2017.

Tabelle 6: $\quad$ DB I (erntekostenfreier Holzerlös) der befragten BB-Forstbetriebe Wirtschaftsjahr 2017 ( $n=38$ ), getrennt nach Baumartengruppen sowie nach Obere 25\%, Durchschnitt aller Betriebe und Untere 25\%, Quelle: BBForstbetriebsvergleich 2017.

Tabelle 7: $\quad$ Berechnung der jährlichen Holzproduktionswerte in $€ /$ ha in Abhängigkeit vom Bestandesalter und dem Bestandesalter am Ende der Betrachtungsperiode für Fichte, I. Ertragsklasse, mäßige Durchforstung (Durchmesserentwicklung adjustiert).

Tabelle 8: Berechnung der jährlichen Holzproduktionswerte in $€ /$ ha in Abhängigkeit vom Bestandesalter und dem Bestandes-alter am Ende der Betrachtungsperiode für Buche, I. Ertragsklasse, mäßige Durchforstung (Durchmesserentwicklung adjustiert und Wertziffernabfall ab Alter 120 Jahre unterstellt)

Tabelle 9: Umrechnungsfaktoren von langfristigen Wirkungszeiträumen in kurzfristige Ausgleichszeiträume (berechnet auf der Basis eines Kalkulationszinses von $1,5 \%)$

Tabelle 10: Jährliche Ertragsverluste in Prozent des Abtriebswertes, in Abhängigkeit von der Höhe des Wertverlustes (Restwert \%) und des Wirkungszeitraums (bei unterstelltem Zins von 1,5\%)

Tabelle 11: Eingangsdaten der verglichenen Kalkulationstools für forstliche Nutzungsbeschränkungen. Zur besseren Lesbarkeit befindet sich diese Tabelle erneut im Anhang 9.1.5.

Tabelle 12: Vergleich Referenzbewertungsfälle der fünf Hauptbaumartengruppen mittels der drei Kalkulationstools, Abweichungen zu Bewertungskonzept der Uni Göttingen in \%.

Tabelle 13: Bewertungskonzept für Einzelbäume, beispielhafte Berechnung einiger Baumarten mit variierenden Wertklassen (blau) inkl. Vergleich zu den 
ermittelten Barwerten je Baum aus 2010 (orange) und Abweichung in \% (grün). Zur besseren Lesbarkeit befindet sich die Tabelle erneut im Anhang 9.1.4 95

Tabelle 14: $\quad$ Auflagen der niedersächsischen Natura 2000-Schutzgebietsverordnung im Wald für die Erhaltungszustände, gekürzt, eigene Darstellung 99

Tabelle 15: $\quad$ Kosten des Einzelbaumerhalts, Probefläche 1 Hämeler Wald Abt. 1 in $€ /$ Baum, Berechnung gem. Bewertungskonzept für Einzelbäume 102

Tabelle 16: $\quad$ Ertragsverlust durch Baumartenwechsel, Probefläche 2 Hämeler Wald Abt. 2 , in $€ /$ ha/a, Berechnung gem. Bewertungskonzept für forstliche Nutzungsbeschränkungen

Tabelle 17: Ertragsverlust durch Verlängerung der Umtriebszeit, Probefläche 3 Hämeler Wald Abt. 3, in €/ha/a 106

Tabelle 18: Zusammenfassung Auswirkungen naturschutzfachlicher Auflagen in der Fallstudie Hämeler Wald, in $€ /$ ha/a

Tabelle 19: Entscheidungskriterien der fünf Konzepte, Quelle: Putensen 2018

Tabelle 20: Bewertungskatalog der Habitatmerkmale von Einzelbäumen, abgewandelt und ergänzt nach Meier (2009). Die Punktzahl eines Habitatbaums entspricht der Summe der Punkte seiner Merkmale, Quelle: Putensen 2018142

\section{Formelverzeichnis}

Formel 1: $\quad$ Annuität der forstlichen Produktion einer Umtriebszeit, Quelle: Möhring et al. 2006

Formel 2: $\quad$ Annuität der forstlichen Produktion kürzerer Zeiträume, Quelle: Möhring et al. 2006

Formel 3: $\quad$ Berechnung des Umrechnungsfaktors für Ausgleichszeitraum, Quelle: eigene Darstellung

Formel 4: $\quad$ Ermittlung des jährlichen Ertragsverlusts in \% des Abtriebswertes unter Berücksichtigung des Zinses, der Laufzeit und der unterstellten Wertminderung, Quelle: eigene Darstellung 


\section{Abkürzungsverzeichnis}

\begin{tabular}{|c|c|}
\hline AgrarZahIVerpflG & Agrarzahlungen-Verpflichtungengesetz \\
\hline AgrarZahIVerpfIV & Agrarzahlungen-Verpflichtungenverordnung \\
\hline$A \cup K M$ & Agrarumwelt- und Klimamaßnahmen \\
\hline$B f N$ & Bundesamt für Naturschutz \\
\hline$B H D$ & Brusthöhendurchmesser \\
\hline BNatschG & Bundesnaturschutzgesetz \\
\hline$C C$ & Cross-Compliance \\
\hline$D g$ & Durchmesser des Grundflächenmittelstamms \\
\hline EAFRD & European agricultural fund for rural development \\
\hline EGFL & Europäischer Garantiefonds für die Landwirtschaft \\
\hline$E K$ & Ertragsklasse \\
\hline ELER & $\begin{array}{l}\text { Europäischer Landwirtschaftsfonds für die Entwicklung des länd- } \\
\text { lichen Raumes }\end{array}$ \\
\hline$E P L R$ & Entwicklungsprogramm des ländlichen Raumes \\
\hline$E Z$ & Erhaltungszustand \\
\hline FFH & Flora-Fauna-Habitat \\
\hline GAK & Gemeinschaftsaufgabe Agrarstruktur und Küstenschutz \\
\hline GAP & Gemeinsame Agrarpolitik \\
\hline HPW & Holzproduktionswert \\
\hline i.V.m. & in Verbindung mit \\
\hline$L K L$ & Leistungsklasse \\
\hline$L R T$ & Lebensraumtyp \\
\hline LSG & Landschaftsschutzgebiet \\
\hline IZ & laufender Zuwachs \\
\hline NDS & Niedersachsen \\
\hline$N L F$ & Niedersächsische Landesforsten \\
\hline NRW & Nordrhein-Westfalen \\
\hline NSG & Naturschutzgebiet \\
\hline VNS & Vertragsnaturschutz \\
\hline WBR & Waldbewertungsrichtlinie \\
\hline$W K L$ & Wertklasse \\
\hline WUKM & Waldumwelt- und Klimamaßnahmen \\
\hline$W Z$ & Wertziffer \\
\hline
\end{tabular}





\section{Einleitung}

\subsection{Problemstellung}

Vertragsnaturschutz im Wald gilt als ein wichtiges umweltpolitisches Instrument zum Erhalt und zur Förderung der biologischen Vielfalt im Wald. Die „Nationale Strategie zur biologischen Vielfalt" (NBS), eine Zukunftsvision der Bundesregierung aus dem Jahr 2007, in der rund 330 Zielvorgaben und 430 konkrete, akteursbezogene Maßnahmen beschrieben werden, enthält das explizite Ziel, den Vertragsnaturschutz im Privatwald auf zehn Prozent der Fläche zu fördern (Bundesministerium für Umwelt, Naturschutz und Reaktorsicherheit (BMU) 2007, S. 32). Gleichzeitig versieht auch das Bundesnaturschutzgesetz in $\S 3$ Abs. 3 vertraglichen Naturschutzvereinbarungen eine vorrangige Prüfpflicht und misst ihnen damit einen hohen Stellenwert bei.

Vertragsnaturschutz im Wald bietet für die Gesellschaft und für Forstbetriebe wesentliche Vorteile. Die teilnehmenden Forstbetriebe werden für die von Ihnen erbrachten Leistungen entlohnt, bzw. für Nutzungsverzichte in ökologisch wertvollen Bereichen kompensiert. Durch das finanzielle Entgelt wird Erbringung von Naturschutzleistungen auch für erwerbswirtschaftlich ausgerichtete Forstbetriebe ökonomisch interessant. Sie werden angeregt, diese Leistungen möglichst effizient zu erbringen (Nielsen et al. 2018, S. 1). Die Öffentlichkeit wird durch den Zwang der Kompensation wegen der Knappheit der Mittel zur Auswahl der Maßnahmen mit dem höchsten Nutzen gezwungen (Moog und Brabänder 1994). Der Gesellschaft entsteht somit ein vergleichsweise hoher Nutzen durch den effizienten Einsatz der Mittel (Nielsen et al. 2018, S. 1). Die auf das Gemeinwohl ausgerichtete Ökologie und die forstbetriebliche Ökonomie werden durch dieses Instrument des Vertragsnaturschutzes ein Stück weit miteinander versöhnt.

Trotz dieser im ersten Moment beidseitig vorteilhaft wirkenden Eigenschaften ist der Vertragsnaturschutz im Wald zum Zeitpunkt der Erstellung dieser Arbeit (immer noch) kein weit verbreitetes Instrument, sondern stellt im deutschen Wald eine Randerscheinung dar (Bundesministerium für Umwelt, Naturschutz und Reaktorsicherheit (BMU) 2017, S. 42). Eine Vielzahl von wissenschaftlichen Arbeiten hat sich in der Vergangenheit mit den Rahmenbedingungen, Ausgestaltungsmöglichkeiten und politischen Einstellungen zum Vertragsnaturschutz im Wald beschäftigt (bspw. Moog und Brabänder 1994; Petz 2005; Frenz 2011; Seintsch et al. 2012; Kettunen 2017). Nach wie vor ist unklar, weshalb trotz mannigfaltiger Bemühungen und vieler Forschungsarbeit, die Schwachstellen aufzeigt, das Instrument des freiwilligen Erhalts von ökologisch wertvollen Zuständen, bzw. die Herstellung oder die Erbringung von Leistungen ge- 
gen Vergütung so wenig Verbreitung findet. Die vorliegende Arbeit will dieser Frage unter besonderer Berücksichtigung eines systematischen Vergleiches der Instrumente in der Landwirtschaft und der Forstwirtschaft und unter Zuhilfenahme von Analysen der Einstellungen von Land- und Forstwirten zu diesem Thema nachgehen. Durch Weiterentwicklung von Instrumenten zur betrieblichen Bewertung der Einschränkungen durch Naturschutzmaßnahmen will sie weiterhin den Prozess der Umsetzung von Vertragsnaturschutz im Wald fördern.

\subsection{Einordnung Wald - Naturschutz - Geld}

\subsubsection{Begriff Vertragsnaturschutz}

Unter Vertragsnaturschutz wird jede vertragliche Vereinbarung zwischen Grundeigentümern, Nutzungsberechtigten oder sonstigen geeigneten Personen und Verwaltung verstanden, die vorrangig der Verwirklichung von Naturschutzzielen durch die honorierte Vornahme, Duldung oder Unterlassung von Maßnahmen dient (Petz 2005). Damit setzt der Vertragsnaturschutz laut Petz (2005) erst dort an, wo er über rechtlich verpflichtende Mindestanforderungen hinaus geht, bzw. ein aktives Handeln für den Naturschutz erfordert. Bei den Verträgen zwischen Verwaltung und Waldbesitzer handelt es sich um subordinationsrechtliche Verträge anstelle von rechtlich zulässigen hoheitlichen Handlungen. Besonders im Bereich der Verträge zwischen Privatpersonen oder Unternehmen und der öffentlichen Hand bestehen immer wieder Abgrenzungsschwierigkeiten hinsichtlich des Begriffs „Vertragsnaturschutz". Es ist umstritten, ob ausschließlich juristisch eindeutige, freiwillige Verträge oder vertragliche Vereinbarungen als Vertragsnaturschutz begriffen werden oder ob auch mitwirkungsbedürftige Verwaltungsakte (bspw. die Inanspruchnahme öffentlicher Förderprogramme) dem Vertragsnaturschutz zugerechnet werden können (Windstoßer 2008, S. 20). Lutter und Paschke (2018, S. 28) plädieren aufgrund des Fehlens einer allgemeingültigen Definition dafür, Vertragsnaturschutz als weitgefasstes Instrument zu verstehen, dass durch die Gewährung eines Entgelts im Gegenzug für die Bereitstellung von Naturschutzleistungen gekennzeichnet wird. Die Entgelte sollen betriebliche Nachteile, die der Betrieb infolge der Maßnahmen erleidet, kompensieren (Paschke 2018, S. 34).

Der Vorteil des Vertragsnaturschutzes wird in der Literatur vor allem in der besseren Akzeptanz der Flächeneigentümer und somit einer höheren Effektivität gegenüber hoheitlichen Handlungen gesehen (Güthler et al. 2005; Frenz 2011). Diese herrschende Meinung konnte in einer Befragung von privaten Waldbesitzern im WaVerNa-Projekt bestätigt werden, an der der Autor dieser Arbeit beteiligt war (Kownatzki et al. 2017). 
Neben dem von Petz (2005) definierten Vertragsnaturschutz im engeren Sinne, werden in Deutschland weitere Instrumente zwischen Waldbesitzern und potenziellen Geldgebern (öffentliche Hand, Stiftungen, Verbände) eingesetzt, um Naturschutzmaßnahmen im Wald umzusetzen. Da diese Instrumente dem eigentlichen Vertragsnaturschutz sehr ähnlich sind, werden sie in dieser Arbeit ebenso wie im WaVerNa-Projekt (s. 2.3) unter dem Begriff "Naturschutz gegen Entgelt" subsumiert. Wenn im Folgenden von Waldnaturschutzmaßnahmen gegen Entgelt, Naturschutz gegen Entgelt oder Vertragsnaturschutz die Rede ist, dann bezieht sich dies stets auf die weiter gefasste Definition.

Zusätzlich zum privat- oder subordinationsrechtlichen Vertragsnaturschutz nach Petz (2005) kommen folgende Instrumente in den Bundesländern zum Einsatz:

- Allgemeine forstliche Förderung (Schwerpunkt Naturschutzmaßnahmen)

- Waldumweltmaßnahmen

- Erschwernisausgleich durch Bewirtschaftungsauflagen

- Sonstige Entgeltzahlungen für Naturschutzmaßnahmen

Der finanziell wichtige Bereich der Ausgleichs- und Ersatzmaßnahmen bei Eingriffen in Naturund Landschaft ( $§ 13 \mathrm{BNatSchG)}$ wird in dieser Arbeit nicht zum Naturschutz gegen Entgelt gezählt. Auf die Ausgleichs- und Ersatzmaßnahmen soll wegen ihrer großen ökonomischen Bedeutung für die Forstbetriebe gleichwohl im folgenden Kapitel 1.2.2 kurz eingegangen werden.

\subsubsection{Ausgleichs- und Ersatzmaßnahmen}

Beeinträchtigungen von Natur und Landschaft sind zu vermeiden ( $§ 13$ BNatSchG). Nicht vermeidbare Beeinträchtigungen sind durch Ausgleichs- und Ersatzmaßnahmen oder Ersatz in Geld zu kompensieren. Ein Ausgleich ist erfolgt, wenn die Funktion des Naturhaushalts in gleichartiger Weise wiederhergestellt wird, Ersatz ist erfolgt, wenn die beeinträchtigte Funktion im gleichen Naturraum in gleichwertiger Weise wiederhergestellt ist (§ 15 BNatSchG Abs. 2).

In §14 Abs. 1 BNatSchG wird der Eingriff folgendermaßen definiert:

„Eingriffe in Natur oder Landschaft im Sinne dieses Gesetzes sind Veränderungen der Gestalt oder Nutzung von Grundflächen oder Veränderungen des mit der belebten Bodenschicht in Verbindung stehenden Grundwasserspiegels, die die Leistungs- und Funktionsfähigkeit des Naturhaushalts oder das Landschaftsbild erheblich beeinträchtigen können." 
Gemäß Abs. 2 des vorgenannten Paragrafen sind die land-, forst- und fischereiwirtschaftliche Bodennutzung nicht als Eingriff anzusehen, solange die Ziele des Naturschutzes und der Landschaftspflege berücksichtigt werden (Privilegierung). Dabei sind die Regeln der guten fachlichen Praxis einzuhalten.

Nach Abs. 3 ist die Wiederaufnahme einer land-, forst- oder fischereiwirtschaftlichen Bodennutzung nach zeitweiser Einschränkung oder Unterbrechung aufgrund vertraglicher Vereinbarungen, Teilnahmen an öffentlichen Programmen oder durch vorgezogene Kompensationsmaßnahmen ebenfalls nicht als Eingriff anzusehen (Wiederaufnahmeprivileg).

Neben den weiteren Vorschriften der $\S 13 \mathrm{ff}$. ist somit bei einem unvermeidbaren Eingriff in Natur und Landschaft die näherungsweise Wiederherstellung einer ursprünglichen Situation geregelt. In der Gesamtbetrachtung ist daher, im Gegensatz zu dem freiwilligen Vertragsnaturschutz, der im Zentrum dieser Arbeit steht, kein naturschutzfachlicher Mehrwert geschaffen, sondern nur nach einem schädigenden Eingriff der Status ante wiederhergestellt.

Aufgrund der reinen Wiederherstellung und der verpflichtenden Durchführung für einen Eingriffsverursacher sind die Ausgleichs- und Ersatzmaßnahmen im Gegensatz zu den freiwilligen Vereinbarungen kein Objekt der weiteren Betrachtung in dieser Arbeit. Dennoch ist davon auszugehen, dass ein Großteil der investiven Naturschutzmaßnahmen in der Land- und Forstwirtschaft durch Ausgleichs- und Ersatzmaßnahmen, bzw. Ersatzgelder finanziert wird. Aufgrund der unterschiedlichen behördlichen Zuständigkeiten in den Bundesländern gibt es nur wenige Informationen über Volumen und Art der eingesetzten Gelder (s. Wätzold und Wissel 2016). Ansätze im WaVerNa-Forschungsvorhaben Umfang und Höhe der jährlichen Ausgleichs- und Ersatzmaßnahmen in den Bundesländern zu erfassen, stießen früh auf methodische und politische Probleme. In diesem, für den Naturschutz so wichtigen Bereich, ist weitere Forschung notwendig. 


\subsection{Ziel und Aufbau der Arbeit}

\subsubsection{Ziel}

Die vorliegende Arbeit verfolgt drei Ziele:

1. Analyse der betriebswirtschaftlichen Aspekte des Vertragsnaturschutzes im Wald in der Vergangenheit und Erhebung des aktuellen Status quo unter Berücksichtigung der Ergebnisse des WaVerNa-Projekts, an dem der Autor beteiligt war und Darstellung der Grundlagen des Vertragsnaturschutzes in der Landwirtschaft als kontrastierendes praktisches Anwendungsbeispiel.

2. Die im Rahmen des WaVerNa-Verbundforschungsvorhabens und durch die allgemeinen Analysen der Regelungen für Agrarumweltmaßnahmen im Bereich der Landwirtschaft erhobenen Erkenntnisse sollen im Hauptteil dieser Arbeit in einer vom Autor durchgeführten Befragung privater Erwerbsforstbetriebe erfassen, welche Stärken und Schwächen die bisherigen Vertragsnaturschutzmaßnahmen in den Bereichen Land- und Forstwirtschaft aufweisen und ob aus den für die Landwirtschaft entwickelten Konzepten Hinweise für die Umsetzung von Vertragsnaturschutz im Forstbereich hilfreich entwickelt werden können.

3. Da die Analysen ergeben, dass die Angemessenheit und Dauer der Zahlung eine Schlüsselfrage bei der Umsetzung von Vertragsnaturschutzmaßnahmen im Wald ist, sollen bestehende Bewertungskonzepte zur Abschätzung von Mehraufwendungen und Mindererträge für typische forstliche Maßnahmen, die für den Vertragsnaturschutz im Wald in Frage kommen, aktualisiert und um neue Maßnahmentypen erweitert werden, um auf aktueller Zahlenbasis Mindestentgelte für Vertragsnaturschutzmaßnahmen im Wald abschätzen zu können. Anschließend werden die aktualisierten Bewertungskonzepte in einer Fallstudie exemplarisch angewandt und so validiert. Weiterhin sollen die bisher meist vernachlässigten Mehraufwendungen im Bereich der Verwaltungs- und Kontrollaufwands aber auch die kritischen Aspekte der Frage der Verkehrssicherheit beim Erhalt von Alt- und Habitatbäumen als häufigstes Beispiel für Vertragsnaturschutmaßnahmen exemplarisch analysiert werden. 


\subsubsection{Aufbau}

Nach der Einleitung erfolgt in Kapitel 2 die Darstellung der Ergebnisse aus der Status quo Analyse des Vertragsnaturschutzes im Wald. Die Diskrepanz in der Vergangenheit zwischen politischen Willenserklärungen und tatsächlicher Umsetzung ist dabei von wichtiger Bedeutung, um viele aktuelle Probleme und Vorbehalte seitens der Waldbesitzer nachzuvollziehen. Im nächsten Block erfolgt eine Analyse und Übersicht der Vielfalt von Finanzierungsquellen, die aus dem deutschen Föderalismus resultiert.

Teile der vorliegenden Arbeit wurden bereits an anderen Stellen veröffentlicht. Dies sind maßgeblich die Zwischen- und Abschlussberichte des WaVerNa-Verbundforschungsvorhabens an die Fachagentur für Nachwachsende Rohstoffe (FNR) und das Sonderheft Vertragsnaturschutz der AFZ-DerWald 21/2018 als Darstellungsmedium der Forschungsergebnisse für die Öffentlichkeit. Die für diese Arbeit wichtigsten, bereits veröffentlichten Ergebnisse werden in den Kapiteln 2.3 und 2.4 zusammenfassend dargestellt. Das Kapitel 2.6 schließt mit einem Exkurs in den landwirtschaftlichen Vertragsnaturschutz als denkbare „Blaupause“ für eine Neuausrichtung des forstlichen Vertragsnaturschutzes ab.

Kapitel 3 befasst sich mit der Befragung privater Erwerbsforstbetriebe bezüglich der Erfahrungen, Probleme und Verbesserungsmöglichkeiten von Vertragsnaturschutzmaßnahmen im Wald. Die Befragung beinhaltet weiterhin einen Vergleich zum Vertragsnaturschutz in der Landwirtschaft.

In Kapitel 4 erfolgt die Aktualisierung und Erweiterung bestehender forstlicher Bewertungskonzepte für Nutzungseinschränkungen und Einzelbaumerhalte. Ergänzt wird das Kapitel um die Analyse von Verteilungskonzepten für die Habitatbaumauswahl sowie eine Anwendung der vorgenannten Bewertungskonzepte im Rahmen einer Fallstudie.

Die Ergebnisse der Blöcke werden thematisch getrennt diskutiert. Dieser Aufbau resultiert aus dem abschnittsweisen, aufeinander aufbauenden Vorgehen und dem hier relevanten Ziel- und Methodenpluralismus, der eine gemeinsame Diskussion unübersichtlich und wenig zielführend erscheinen lässt.

In Kapitel 6 erfolgt dann eine Zusammenführung der wesentlichen Erkenntnisse der einzelnen Kapitel, um Schlussfolgerungen für den Vertragsnaturschutz in Wald in seiner aktuellen Form und einen Ausblick auf mögliche Lösungen für zukünftige Ansätze zu geben und die Notwendigkeit weiterführender Forschung zu diesem Thema aufzuzeigen. 


\section{Status quo Vertragsnaturschutz im Wald}

\subsection{Rahmenbedingungen für den Vertragsnaturschutz im Wald}

\subsubsection{Politische Willenserklärungen und tatsächliche Handlungen}

Das Bundesnaturschutzgesetz (BNatSchG) räumt dem Vertragsnaturschutz in $\S 3$ Abs. 3 einen hohen Stellenwert ein (Lutter und Paschke 2018). „Bei Maßnahmen des Naturschutzes oder der Landschaftspflege soll vorrangig geprüft werden, ob der Zweck mit angemessenem Aufwand auch durch vertragliche Vereinbarungen erreicht werden kann" (Deutscher Bundestag 2009). Zwar besteht kein genereller Vorrang vertraglicher Vereinbarungen vor anderen Instrumenten, die zuständigen Behörden sind jedoch zur Überprüfung vertraglicher Lösungen angehalten. Es kommt die Erwünschtheit konsensualer Vereinbarungen deutlich zum Ausdruck (Lutter und Paschke 2018). Auch die Tatsache, dass ein Vertrag regelmäßig das mildere Mittel staatlichen Handelns als ein ordnungsrechtlicher Verwaltungsakt darstellt, unterstreicht die Überzeugung des Gesetzgebers eine solche Prüfpflicht zu formulieren (Frenz 2011). Erneut aufgegriffen wird dieser Gedanke im $\S 32$ Abs. 4 BNatSchG, der vertraglichen Regelungen zum Schutz von Natura 2000-Gebieten bei gleichwertiger Schutzgewährleistung einen ebenso hohen Stellenwert wie ordnungsrechtlichen Instrumenten zugesteht.

Jedoch werden dieser Möglichkeit durch die Europäische Rechtsprechung enge Grenzen gesetzt (EuGH, Urteil vom 25.11.1999). Diese hat mehrfach entschieden, dass vertragliche Vereinbarungen allein für den Schutz naturschutzfachlich wertvoller Gebiete (bspw. Natura 2000) nicht ausreichend sind.

Jenseits der rechtlichen Sicherung von Schutzgebieten kann der Vertragsnaturschutz grundsätzlich eingesetzt werden, um naturschutzfachlich wertvolle Zustände zu sichern oder herzustellen. Die Europäische Union hat hierzu in der aktuellen und vorangegangenen ELER-Förderperiode ${ }^{1}$ verschiedene Grundsteine für eine Umsetzung solcher Maßnahmen in den Programmen der Mitgliedsländer gelegt. Grundsätzlich stellt die ELER-Förderung neben der GAK ${ }^{2}$ das zentrale Instrument der Naturschutzfinanzierung sowohl in der Land- als auch in der Forstwirtschaft in Deutschland dar (Hansjürgens et al. 2019, S. 64).

\footnotetext{
${ }^{1}$ Europäischer Landwirtschaftsfonds für die Entwicklung des ländlichen Raumes

${ }^{2}$ Gemeinschaftsaufgabe Agrarstruktur und Küstenschutz (vgl. Bundesministerium für Ernährung und Landwirtschaft 2019)
} 


\subsubsection{ELER-Förderperiode 2007 - 2013}

In der Förderperiode 2007-2013 wurden von den Bundesländern unterschiedliche Ansätze von Naturschutz gegen Entgelt auf Basis der Europäischen Förderpolitik durchgeführt. Zumeist wurden die Maßnahmen durch EU-Fördermittel kofinanziert (Entenmann und Schaich 2014). Eine Ausnahme stellte das Bundesland Hessen dar, dessen Vertragsnaturschutzprogramm rein auf Länderebene finanziert wurde (HMUELV 2009). In der ELER-VO waren für den Naturschutz gegen Entgelt im Wald vor allem die Artikel 46 (Zahlungen im Rahmen von Natura 2000 im Wald, Code 224), Artikel 47 (Zahlungen für Waldumweltmaßnahmen, Code 225) und Artikel 49 (Beihilfen für nichtproduktive Investitionen im Wald, Code 227) maßgeblich (Entenmann und Schaich 2014). Die Kofinanzierungsanteile der EU beliefen sich für diese Artikel auf $55 \%$ in den alten Bundesländern und auf 80 \% in den neuen Bundesländern (Deutsche Vernetzungsstelle Ländliche Räume 2008).

Einzelne Bundesländer, bspw. Niedersachsen, haben in der Vergangenheit versucht mittels ELER-Mitteln im Wald ein paralleles Instrument zu den Agrarumweltmaßnahmen umzusetzen. Die sogenannten Waldumwelt- und Klimamaßnahmen (WUKM) sind in der Europäischen Gesetzgebung klar vorgesehen, bedürfen aber wie alle Maßnahmen einer konkreten Umsetzung in regionalen Entwicklungsprogrammen. Der Versuch erwies sich in Niedersachsen als Sackgasse. Nach der Hälfte der Laufzeit des ELER-Programms 2007 bis 2013 wurden die für WUKM bereitgestellten Mittel abgezogen und an anderer Stelle eingesetzt. Als größtes Problem erwies sich die aufwendige Verwaltungsarbeit und die mangelnde Akzeptanz durch die Waldbesitzer, die bereits im Vorfeld prognostiziert wurden (Grohe 2010).

Europaweit sind in der ersten Hälfte (2007-2010) der ELER-Förderperiode 2007-2013 lediglich 0,3 \% der zur Verfügung stehenden Mittel (ELER und nationale Mittel) für Waldumweltmaßnahmen abgerufen worden (Kettunen et al. 2012 in Entenmann und Schaich 2014). Im Gegensatz dazu betrug der Anteil der abgerufenen Mittel für Agrarumweltmaßnahmen 24,4 \% (Entenmann und Schaich 2014). Bis 2013 stiegen die für forstliche Maßnahmen in Anspruch genommenen Mittel nur auf 4,2 \% (Szedlak 2013 in Entenmann und Schaich 2014).

Von den bundesweit abgerufenen 17,9 Mrd. € des Zeitraums 2007-2013, bestehend aus ELERMitteln (9 Mrd. €), nationalen Mitteln (5,6 Mrd. €) und sog. „Top-ups“ der Länder (3,3 Mrd. €), waren lediglich 112,5 Mio. € (0,6 \%) für Maßnahmen des Naturschutzes im Wald vorgesehen (Entenmann und Schaich 2014). 


\subsubsection{ELER-Förderperiode $2014-2020$}

Auch die aktuelle Förderperiode der Gemeinsamen Agrarpolitik der Europäischen Union basiert auf den zwei Säulen, die seit der Agenda 2000 aus dem Jahr 1999 so bezeichnet werden. Die erste Säule ${ }^{3}$ umfasst einerseits die Direktzahlungen der EU an die landwirtschaftlichen Betriebe, wobei ein Teil der Zahlungen erstmals an die Einhaltung gewisser ökologischer Standards gebunden ist („Greening-Komponente“) und andererseits die Marktmaßnahmen für Interventionen und sektorspezifische Unterstützungen ${ }^{4}$.

Daraus ergibt sich die in Abbildung 1 dargestellte Aufteilung der landwirtschaftlichen Direktzahlungen für die Betriebe. Die stärkere Förderung von Junglandwirten und kleinen Betrieben (Zusatzförderung erste Hektare) führt zu unterschiedlichen Prämienhöhen in Abhängigkeit des Alters des Betriebsleiters und der Größe des Betriebs. Im Durchschnitt fließen rund $294 € /$ ha Direktzahlungen den Betrieben zu (Volckens 2014).

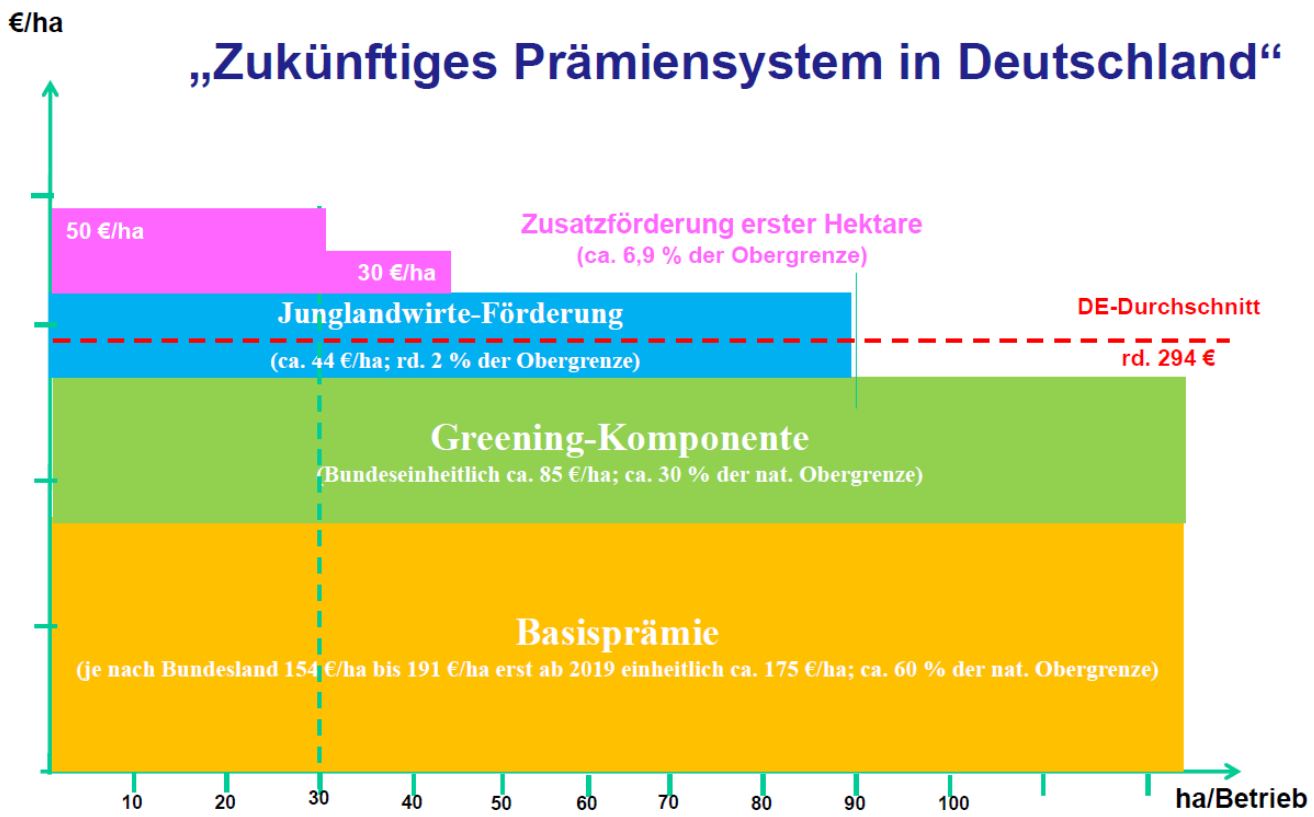

Abbildung 1: Grafische Darstellung aktuelles Prämiensystem der 1. Säule der GAP in Deutschland, inkl. Betriebsgrößenberücksichtigung, Quelle: Volckens 2014

Traditionell überwiegt die Finanzausstattung der ersten Säule, die in der Förderperiode 2014 bis 2020 insgesamt rund $34 \mathrm{Mrd}$. € für deutsche Landwirte beträgt (s. Abbildung 2). Die zweite Säule

\footnotetext{
${ }^{3}$ Finanziert aus dem Europäischen Garantiefonds für die Landwirtschaft (EGFL)

${ }^{4}$ Diese Maßnahmen sollen die Agrarmärkte stabilisieren oder Nachfrage anregen. Bei öffentlichen Interventionen können die Regierungen der EU-Länder Erzeugnisse aufkaufen, einlagern und zu einem späteren Zeitpunkt vermarkten, um ein Überangebot zu verhindern (Europäische Kommission 2020).
} 
zur Förderung der ländlichen Entwicklung und der Finanzierung von Agrarumwelt- und Klimamaßnahmen umfasst unter Berücksichtigung der nationalen Kofinanzierung ein Volumen von rund 17 Mrd. €.

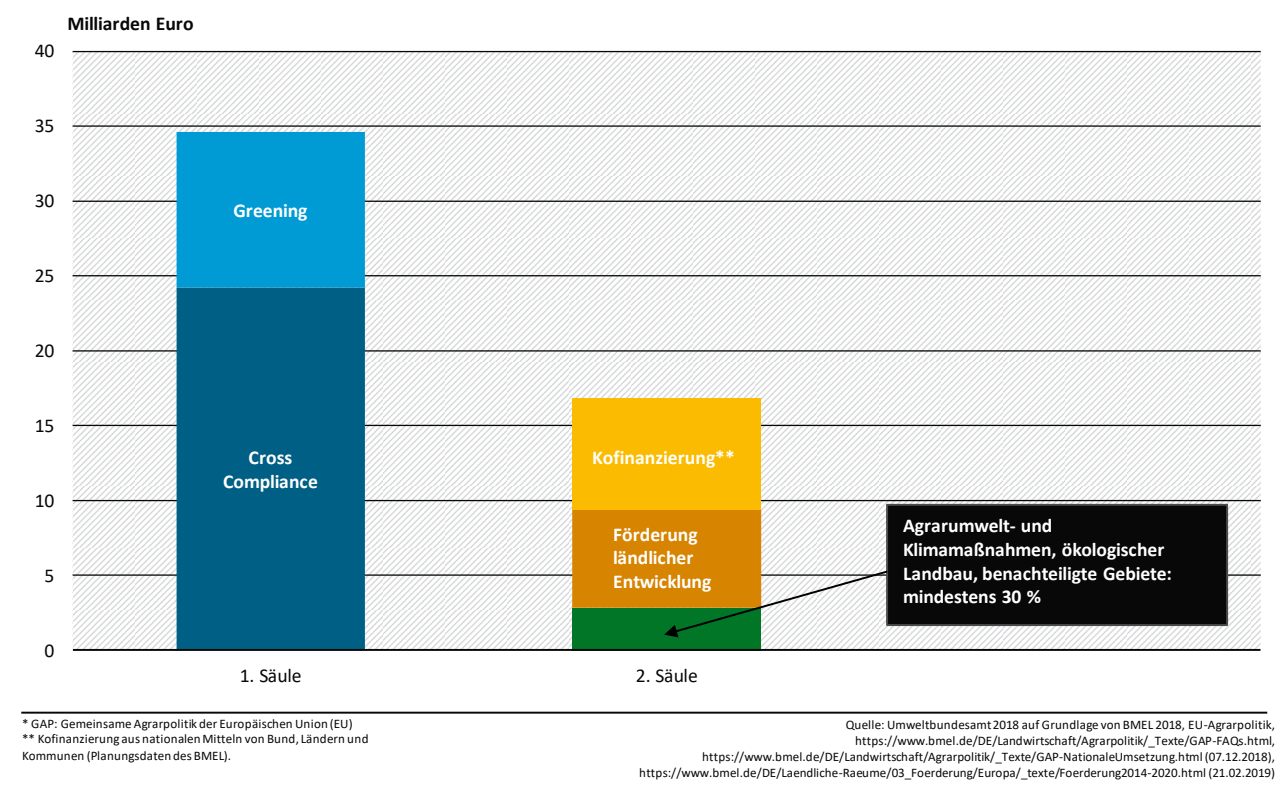

Abbildung 2: GAP-Mittel für Deutschland 2014 bis 2020, Quelle: Umweltbundesamt 2018 auf Grundlage BMEL 2018

Die zweite Säule (ELER) umfasst die Ziele und Maßnahmen zur Förderung des ländlichen Raumes. Neben landwirtschaftlichen und ländlichen Maßnahmen sind auch Zahlungen für ökologische Maßnahmen im Wald grundsätzlich wieder vorgesehen.

In der aktuellen ELER-Förderperiode sind durch die europäische Gesetzgebung in der EU VO 1305/2013 (Europäisches Parlament; Rat der Europäischen Union 2013a) erneut Möglichkeiten zur Finanzierung des Naturschutzes im Wald gegen Entgelt unter Art. 30 Zahlungen für Natura 2000-Gebiete und Art. 34 Waldumwelt- und Klimadienstleistungen und Erhaltung der Wälder gegeben.

Diese werden in Deutschland jedoch nur von einzelnen Bundesländern umgesetzt. Während Thüringen die Waldumweltmaßnahmen aus Art. 34 der EU-Verordnung 1305/2013 in den Katalog der forstlichen Förderung aufgenommen hat (vgl. Thüringer Ministerium für Infrastruktur und Landwirtschaft 2019) und Mecklenburg-Vorpommern nach Art. 30 der EU-Verordnung 1305/2013 die Zahlungen für Natura 2000-Gebiete aus ELER kofinanziert (Ministerium für Landwirtschaft, Umwelt und Verbraucherschutz Mecklenburg-Vorpommern 2016), verwenden Bundesländer wie Hessen (vgl. Hessisches Ministerium für Umwelt, Landwirtschaft und Forsten et al. 2002) oder Bayern (vgl. Bayrisches Staatsministerium für Ernährung, Landwirtschaft und Forsten 2014; Güthler et al. 2018 ) ausschließlich landeseigene Mittel. Auch auf europäischer 
Ebene wurden die vorgesehen ELER-Mittel nicht im erwarteten Umfang für Waldnaturschutz abgerufen (Buren et al. 2016, S. 270). Die Waldumwelt- und Klimamaßnahmen sind in der ELERFörderperiode 2014-2020 aufgrund ihrer kurzen Laufzeiten und den europäischen Kontrollvorgaben mit den gleichen Problemen wie in der vorhergegangen Periode versehen (Freese 2016). Dazu zählt in Deutschland unter anderem auch das Fehlen eines einheitlichen forstlichen Flächenverzeichnisses, dass den europäischen Vorgaben genügt (Bormann 2015, S. 9).

\subsubsection{ELER-Förderperiode $2021-2027$}

Zum Zeitpunkt der Erstellung dieser Arbeit (Herbst 2020) ist die ELER-Periode 2014 - 2020 beinahe abgelaufen. Die Ausgestaltung der zukünftigen Prämienstruktur ist Gegenstand der laufenden Diskussionen. Nachfolgende Aspekte haben sich bislang herauskristallisiert:

Die Gemeinsame Agrarpolitik der Europäischen Union wird zukünftig noch stärker auf den Schutz der Biodiversität und der natürlichen Ressourcen sowie den Klimaschutz ausgerichtet sein (Ministerium für Landwirtschaft, Umwelt und Klimaschutz Brandenburg, 2020). Unter dem Begriff der „Grünen Architektur“ werden die Elemente Konditionalität, Eco Schemes und die Agrarumwelt- und Klimamaßnahmen zusammengefasst. Die Konditionalität beinhaltet Regelungen bei der Flächenbewirtschaftung, die grundsätzlich für die Inanspruchnahme von Mitteln der 1. oder 2. Säule einzuhalten sind. Die Eco Schemes sind freiwillige Maßnahmen aus Mitteln der 1. Säule, die in der bisherigen Förderperiode als bspw. „Greening-Auflagen“ bezeichnet wurden. Aus ökologischer Wertsicht darüber rangieren die Agrarumwelt- und Klimamaßnahmen (AUKM) der 2. Säule. In Abweichung zu den bisherigen landeseigenen ELER-Programmen ${ }^{5}$ wird es nur noch einen nationalen Strategieplan geben, der sowohl die Regelungen der 1. Säule als auch der 2. Säule umfasst, innerhalb dessen bundeslandesspezifische AUKM angelegt werden können (Niedersächsisches Ministerium für Ernährung, Landwirtschaft und Verbraucherschutz 2020). Auch neuartige Maßnahmen, wie z.B. Agroforstsysteme könnten mit aufgenommen werden.

Nach aktuellen Vorschlägen ist jedoch mit einer deutlichen Mittelkürzung der Gemeinsamen Agrarpolitik (GAP), vor allem den Mitteln der 2. Säule (ELER) zu rechnen. Durch eine stärkere Verschiebung der Mittel in die 1. Säule zur Abdeckung der zusätzlichen Umweltauflagen, verknappt sich die Finanzausstattung für freiwillige AUKM in der 2. Säule. Die Folgen für Waldumwelt- und Klimamaßnahmen (WUKM) sind noch nicht absehbar, es ist jedoch auf Grundlage der bisherigen Förderentwicklung nicht davon auszugehen, dass es zu nennenswerten Erhöhungen der veranschlagten Fördermittel kommen wird.

\footnotetext{
${ }^{5}$ Bspw.: PFEIL in Niedersachsen, HALM in Hessen
} 


\subsection{Länder- und Instrumentenvielfalt}

Die föderale Struktur der Bundesrepublik Deutschland führt in Verbindung mit der Regionalität der ELER-Förderprogramme zu einer Vielzahl von denkbaren und umgesetzten Kombinationen von Finanzierungsvarianten von AUKM, WUKM sowie weiterer Naturschutzförderangebote. Das nachfolgende Kapitel soll zusammenfassend einen Überblick über die in der Praxis vorzufindenden Finanzierungsvarianten von Vertragsnaturschutz im Wald geben.

\subsubsection{Finanzierungsvarianten}

Die Finanzierung bestehender Maßnahmen im Bereich des Waldnaturschutzes gegen Entgelt in Deutschland erfolgt maßgeblich durch vier Varianten. Drei dieser Varianten bestehen aus KoFinanzierungsmechanismen mehrerer Institutionen, eine bedient sich ausschließlich landeseigener Mittel (Selzer 2018).

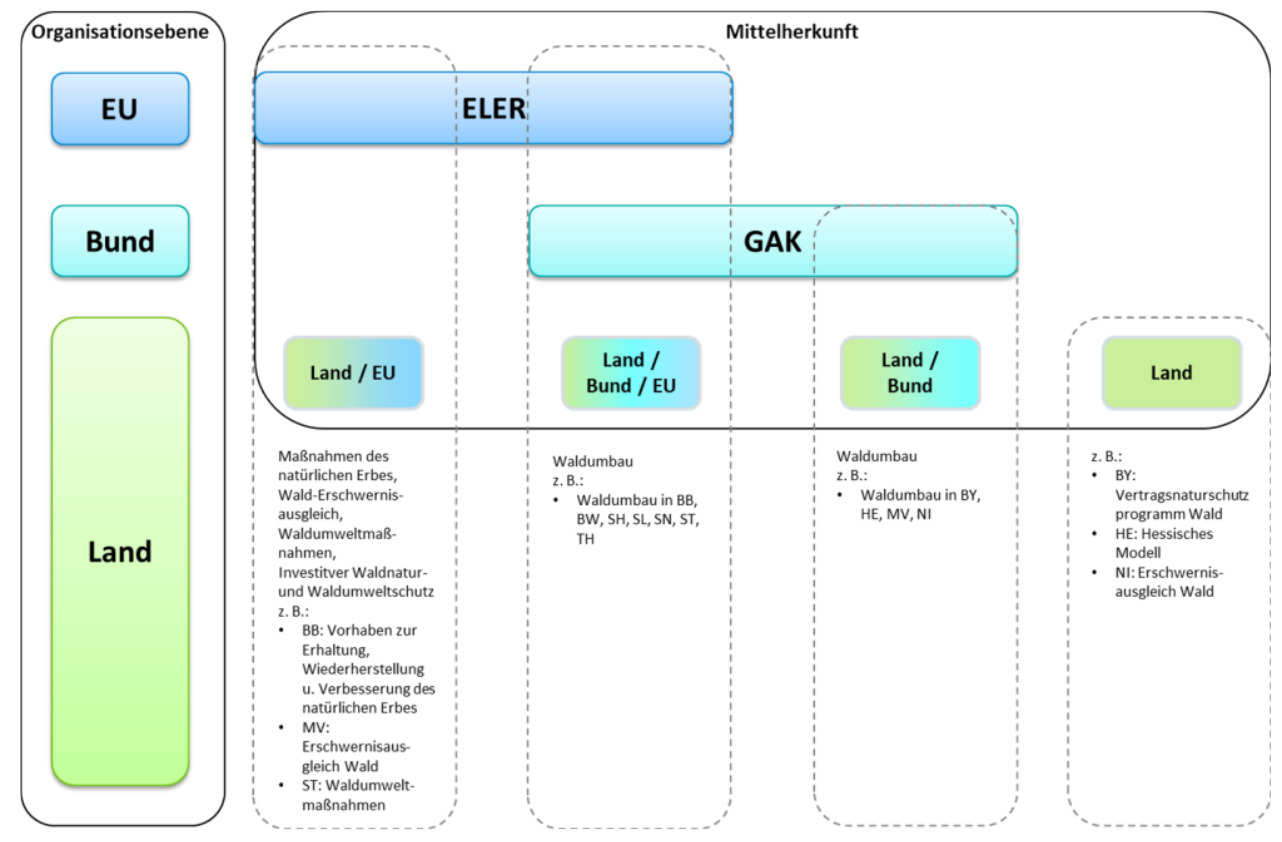

Abbildung 3: Finanzierungsvarianten für Waldnaturschutz gegen Entgelt, Quelle: Selzer 2018

\subsubsection{Alleinfinanzierung}

Die Alleinfinanzierungsvariante wird bspw. vom Bundesland Hessen mit dem „Rahmenvertrag Vertragsnaturschutz im Wald“ (Hessischer Waldbesitzerverband et al. 2002b) umgesetzt, der zwischen dem Land Hessen, dem Hessischen Waldbesitzerverband sowie dem Hessischen Städte- und Gemeindetag abgeschlossen wurde. Inhalt des Rahmenvertrags ist der finanzielle Ausgleich der aus den Bewirtschaftungseinschränkungen von Natura 2000 Flächen resultierenden Einbußen der Waldbesitzer. Auf Grundlage des Rahmenvertrags können die betroffenen 
Waldbesitzer und Kommunen Einzelverträge mit einer landeseigenen Stiftung Natura 2000 abschließen (Hessischer Waldbesitzerverband et al. 2002a).

Das Bundesland Bayern hat ebenfalls für den Wald ein ausschließlich aus Landesmitteln finanziertes Vertragsnaturschutzprogramm ${ }^{6}$ aufgelegt (s. Bayrisches Staatsministerium für Ernährung, Landwirtschaft und Forstwirtschaft 2014). Auf Grundlage dieser Richtlinie können Waldbesitzer Förderbeträge für die im Programm enthaltenen Naturschutzmaßnahmen beantragen. Ähnlich zum Hessischen Modell sind die Maßnahmen auf die Natura 2000 Kulisse beschränkt.

Auch Niedersachsen hat zum Ausgleich der Bewirtschaftungserschwernisse aus den Naturschutzgebietsauflagen der Natura 2000 Gebiete eine Richtlinie veröffentlicht (Niedersächsische Landesregierung 2016). Die finanziellen Mittel stammen ausschließlich aus dem Landeshaushalt. Diese Ausgleichszahlungen haben allerdings keinen Vertragsnaturschutzcharakter, da sie für obligate Regelungen in Natura 2000-Gebieten, die per Naturschutzgebietsverordnung geschützt wurden, gewährt werden. Freiwillig ist dabei lediglich die Inanspruchnahme durch die Waldbesitzer.

\subsubsection{Zweistufige Ko-Finanzierung Bund-Land}

Auf Grundlage der Gemeinschaftsaufgabe Agrarstruktur und Küstenschutz (GAK) ${ }^{7}$ der Bundesrepublik wird in einigen Bundesländern ein zweistufiges Kofinanzierungsmodell verwendet, bei dem sowohl Bundes- als auch Landesmittel zum Einsatz kommen.

Die GAK ist in Deutschland ein wesentliches Element der Nationalen Strategie für die Entwicklung ländlicher Räume (Niedersächsisches Ministerium für Ernährung, Landwirtschaft und Verbraucherschutz 2018). Ziel ist die Gewährleistung einer leistungs- und zukunftsfähigen Land- und Forstwirtschaft, deren Wettbewerbsfähigkeit im Gemeinsamen Markt der Europäischen Union zu sichern sowie die Förderung ländlicher Gebiete.

In Ausführung des GAK-Gesetzes erstellt der GAK-Planungsausschuss ${ }^{8}$ Rahmenpläne der mehrjährigen Finanzplanung zur Erreichung der vorgenannten Ziele. In diesen Rahmenplänen sind die Fördermaßnahmen sowie die jeweilige Förderungsart enthalten. In Teil II des Rahmenplans enthalten die Förderbereiche 1 bis 4 dabei Maßnahmen zur Stärkung einer umweltgerechten Landwirtschaft, einschließlich Vertragsnaturschutz- und Landschaftspflegemaßnahmen, sowie die

\footnotetext{
${ }^{6}$ VNPWaldR Bayern

7 Basierend auf dem Gesetz über die Gemeinschaftsaufgabe „Verbesserung der Agrarstruktur und des Küstenschutzes" (Deutscher Bundestag 11.10.2016)

${ }^{8}$ Bestehend aus dem BMEL, dem BMF sowie den sechzehn für Agrarstruktur und Küstenschutz zuständigen Landesministern.
} 
Verbesserung der Vermarktungsstrukturen land- und fischereiwirtschaftlicher Erzeugnisse. Der Förderbereich 5 enthält die forstbezogenen Fördermaßnahmen, die in die Bereiche (A) Naturnahe Waldbewirtschaftung, (B) Forstwirtschaftliche Infrastruktur, (C) Forstwirtschaftliche Zusammenschlüsse, (D) Erstaufforstung, (E) Vertragsnaturschutz im Wald und (F) Förderung von Maßnahmen zur Bewältigung der durch Extremwetterereignisse verursachten Folgen im Wald unterteilt sind (Niedersächsisches Ministerium für Ernährung, Landwirtschaft und Verbraucherschutz 2018). Der Förderbereich 6 beinhaltet die auf Gesundheit und Robustheit landwirtschaftlicher Nutztiere bezogenen Maßnahmen, die Förderbereiche 7 bis 9 wiederum beziehen sich auf wasserwirtschaftliche Maßnahmen, den Küstenschutz und die Förderung benachteiligter Gebiete.

Die für den Vertragsnaturschutz im Wald relevanten Fördermaßnahmen unter 5 (E) des Rahmenplans dienen dem „Zweck des Schutzes, der Erhaltung und Wiederherstellung von Lebensräumen und Lebensstätten wildlebender Tier- und Pflanzenarten im Wald sowie [der] Verbesserung der lebensraumtypischen biologischen Vielfalt der Waldökosysteme" (Niedersächsisches Ministerium für Ernährung, Landwirtschaft und Verbraucherschutz 2018). Förderfähig ist die Bewirtschaftung, die Pflege oder der Nutzungsverzicht auf forstwirtschaftlich genutzten Flächen. Die Höhe der Zuwendungen bestimmt sich nach den durch die naturschutzfachlichen Auflagen der Maßnahme, einschließlich des Nutzungsverzichts, zu erwartenden Einkommensverlusten und zusätzlichen Kosten im Vergleich zur bisherigen Bewirtschaftung oder den Kosten der Beibehaltung der Bewirtschaftung. Die Konkretisierung der Vorgaben des Rahmenplans obliegt den Ländern.

Die Umsetzung der Bund-Länder-kofinanzierten Maßnahmen erfolgt durch die Länder in Form von Richtlinien zur allgemeinen forstlichen Förderung (vgl. Niedersächsisches Ministerium für Ernährung, Landwirtschaft und Verbraucherschutz 2018). Abweichend vom GAK-Rahmenplan können die Länder darauf verzichten einzelne Fördermaßnahmen umzusetzen. Dies ist aktuell bspw. für Vertragsnaturschutzmaßnahmen im Wald in Niedersachsen der Fall.

\subsubsection{Dreistufige EU-Bund-Länder-Kofinanzierung}

Zusätzlich zu Förderprogrammen, die nur aus den Mitteln der Länder und des Bundes auf Basis des GAK-Rahmenplans finanziert werden, können Fördermaßnahmen aufgelegt werden, die im Rahmen einer dreistufigen Finanzierung auch Mittel der Europäischen Union aus dem ELER-Topf enthalten. Maßgeblicher Fördertatbestand sind dabei Maßnahmen des Waldumbaus, so z. B. umgesetzt in den Bundesländern Brandenburg, Baden-Württemberg, Schleswig-Holstein, Saarland, Sachsen, Sachsen-Anhalt \& Thüringen (Selzer 2018). 


\subsubsection{Zweistufige EU-Länder-Kofinanzierung}

Die von den Bundesländern auf Grundlage der europäischen Rahmenregelung entwickelten, vorgelegten und umgesetzten regionalen Entwicklungsprogramme (EPLR) verfügen über europäische Fördermittel, die zu einem Teil von den teilnehmenden Bundesländern kofinanziert werden müssen. Üblicherweise beläuft sich der Anteil auf 50 \%. Die Zahlungen für Erschwernisse in Natura 2000-Gebieten und die Waldumwelt- und Klimamaßnahmen (WUKM) nach den Artikeln 30 und 34 der EU-VO 1305/2013 (Europäisches Parlament; Rat der Europäischen Union 2013a) basieren auf dieser Kofinanzierung. In Mecklenburg-Vorpommern wird mit dem Erschwernisausgleich Wald und in Baden-Württemberg mit der Umweltzulage Wald der Artikel 30 umgesetzt. Die Waldumwelt- und Klimamaßnahmen nach Artikel 34 werden aktuell nur in SachsenAnhalt und Thüringen umgesetzt. Insgesamt entsteht somit ein vielfältiges Bild der Umsetzung und Finanzierung von Naturschutzmaßnahmen im Wald in Deutschland. Jede Art ist mit eigenen Herausforderungen für die Verwaltungen und die Grundeigentümer behaftet.

\subsection{Stand der Umsetzung von Vertragsnaturschutz im Wald in Deutsch- land}

Ziel des Verbundforschungsprojekts "Vertragsnaturschutz im Wald“ (kurz: WaVerNa-Projekt, Förderkennzeichen 22007115) war die Erhebung des Status quo zur Umsetzung von Vertragsnaturschutz im Wald in Deutschland, die waldökologische, ökonomische und rechtliche Analyse der Potenziale und Hemmnisse des Vertragsnaturschutzes sowie die Erarbeitung von Handlungsempfehlungen für die politisch angestrebte Steigerung des Waldvertragsnaturschutzes und die Bereitstellung von konkreten Praxishilfen (Johann Heinrich von Thünen-Institut et al. 2019). Das Verbundprojekt wurde vom Thünen-Instituts Hamburg koordiniert, weitere Teilnehmer waren die Universität Hamburg, die Nordwestdeutsche Forstliche Versuchsanstalt sowie die Abteilung für Forstökonomie der Universität Göttingen ${ }^{9}$. Die Laufzeit des Projekts war vom 01.10.2015 bis zum 31.12.2018.

\footnotetext{
${ }^{9}$ Der Autor dieser Arbeit war während der Laufzeit des Projektes wissenschaftlicher Mitarbeiter an der Abteilung für Forstökonomie der Universität Göttingen und mit der Wahrnehmung der forstökonomischen Forschungsaufgaben betraut. Die nachfolgenden Ergebnisse sind Bestandteil seiner Forschungsarbeit im Rahmen des WaVerNa-Projekts.
} 


\subsubsection{Methodik}

Zur Erfassung des aktuellen Umsetzungstands von Naturschutz gegen Entgelt im Wald in Deutschland hat das WaVerNa-Projekt im ersten Schritt im Herbst 2016 eine bundesweite, offene Onlinebefragung von Waldbesitzern als potenziellen „Anbietern“ von Naturschutzleistungen gegen Entgelt durchgeführt (Kownatzki et al. 2017). Waldbesitzer werden dabei als Anbieter begriffen, da sie über das ökologisch wertvolle Objekt Wald verfügen und dessen Leistungen potenziellen Nachfragern gegen Entgelt bereitstellen können. Ziel der Befragung war, einen Überblick über die Umsetzung von Waldnaturschutz gegen Entgelt aus Forstbetriebssicht zu erlangen (Kownatzki et al. 2017). Konkret wurden Fragen zu fünf Themenbereichen gestellt:

1. Durchgeführte Naturschutzmaßnahmen und Instrumente der Umsetzung

2. Erfolgskontrolle

3. Vertragsverhältnisse, -inhalte und -gestaltung

4. Verwaltungsaufwand sowie

5. Einschätzungen zu Stärken und Schwächen sowie Chancen und Risiken von Waldnaturschutzmaßnahmen gegen Entgelt.

Zielgruppe der Befragung waren Betriebe, die in den vergangenen 10 Jahren Waldnaturschutzmaßnahmen gegen Entgelt umgesetzt haben. Eine zweite Zielgruppe waren Betriebe mit Umsetzungsabsichten. Zuvor durchgeführte offene Onlinebefragungen des Thünen-Instituts zeigten, dass private Forstbetriebe über derartige Erhebungsverfahren erreicht werden können (Seintsch et al. 2018). Die Verbreitung des Aufrufs zur Teilnahme über Newsletter und Mitgliedsjournale der Verbände ließ eine erhöhte Teilnahmebereitschaft erwarten (Jackob et al. 2009). Die Fragen wurden vorwiegend geschlossen (mit vorgegebenen Antwortkategorien) formuliert, um den Befragungsaufwand gering zu halten. Offene Fragen dienten nur zur Erhebung weiterführender Informationen. Zwingende Weichenfragen für den weiteren Beantwortungsverlauf ermöglichten eine Gruppierung der teilnehmenden Forstbetriebe (Kownatzki et al. 2017).

Die Onlinebefragung wurde im Zeitraum vom 01.09.2016 bis zum 07.10.2016 über den Internetauftritt des WaVerNa-Projekts durchgeführt. Als offene Onlineumfrage ist die Befragung nicht als repräsentative Stichprobe zur Umsetzung von Waldnaturschutz gegen Entgelt zu bewerten (Kownatzki et al. 2017), da ein repräsentatives Stichprobendesign fehlte. 


\subsubsection{Ergebnisse}

Der Fragebogen wurde von 268 Teilnehmern vollständig ausgefüllt und abgesandt. Bereinigt um Doppelzählungen und Unplausibilitäten verblieben am Ende 195 auswertbare Datensätze. Dabei konnten insgesamt 116 Betriebe mit Umsetzungserfahrungen (davon 33 mit der Beabsichtigung der zukünftigen Fortführung) und 79 Betriebe ohne bisherige Erfahrung aber zukünftiger Absicht identifiziert werden (s. Abbildung 4).

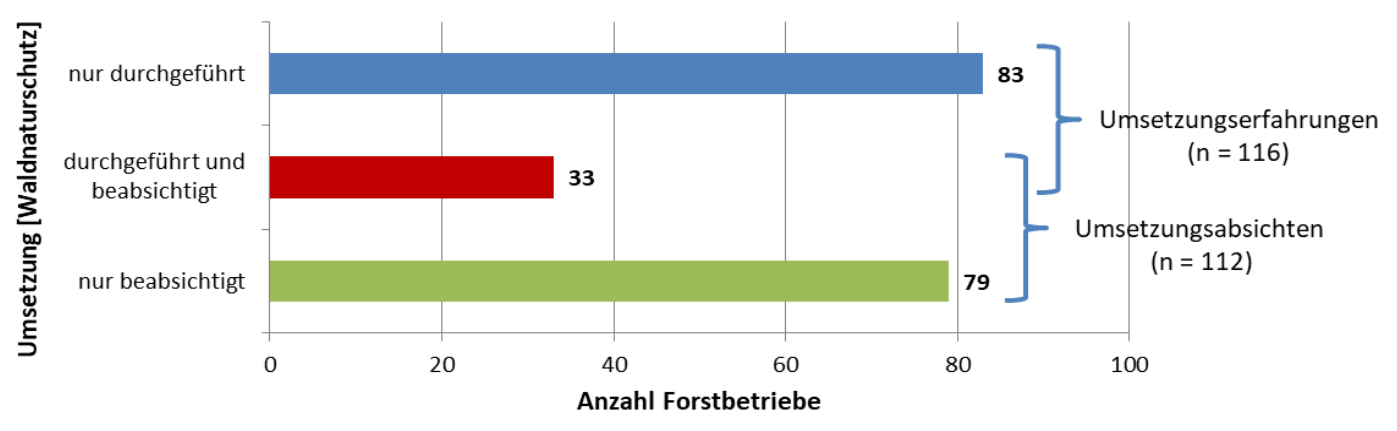

Abbildung 4: Forstbetriebe der Onlinebefragung mit und ohne Umsetzungserfahrungen von Waldnaturschutz gegen Entgelt, Quelle: Kownatzki et al. 2017

Die überwiegende Anzahl der Forstbetriebe mit Umsetzungserfahrungen bei Waldnaturschutz gegen Entgelt lag in Bayern (s. Abbildung 5). Es ist nicht auszuschließen, dass dieser Umstand aus der Vielfalt der Bezeichnungen und Umsetzungsarten für Vertragsnaturschutz resultiert. Das Bayrische Vertragsnaturschutzprogramm trägt als einziges konkret diesen Namen und könnte daher besonderen Einfluss auf die Teilnahmebereitschaft der Waldbesitzer gehabt haben.

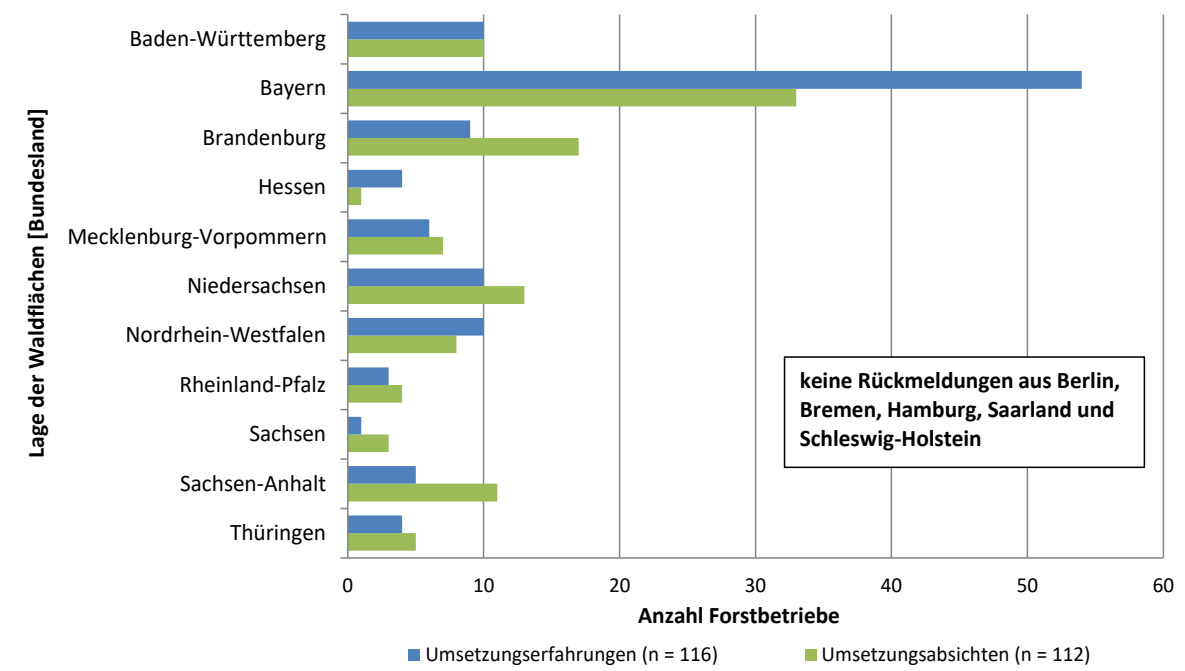

Abbildung 5: Forstbetriebe der Onlineumfrage mit und ohne Umsetzungserfahrungen nach Bundesländern, Quelle: Kownatzki et al. 2017

Lediglich zwei der 116 Forstbetriebe mit Umsetzungserfahrungen habe Waldnaturschutz gegen Entgelt außerhalb einer Schutzgebietskulisse umgesetzt (drei Betriebe keine Angabe). 
Die übrigen Betriebe waren überwiegend an die Natura 2000-Gebietskulisse oder an Naturschutzgebiete gebunden (s Abbildung 6).

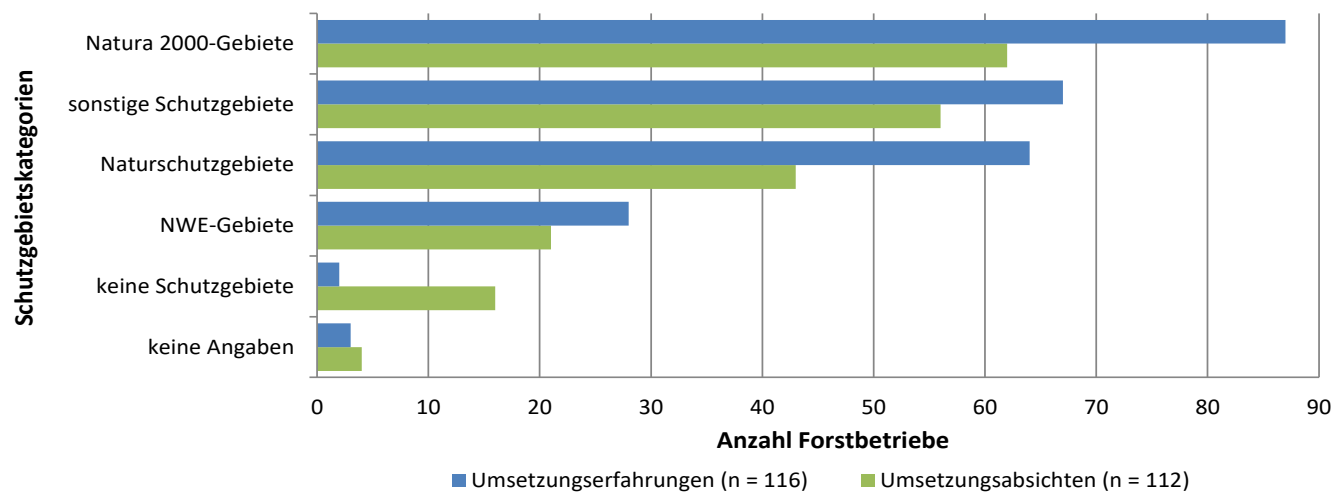

Abbildung 6: Umgesetzte Waldnaturschutzmaßnahmen gegen Entgelt nach Schutzgebietskategorien der Onlineumfrage, NWE = Natürliche Waldentwicklung, Quelle: Kownatzki et al. 2017

Die häufigste Waldnaturschutzmaßnahme gegen Entgelt, die in der Onlineumfrage genannt wurde, war der Erhalt von Alt- und Habitatbäumen (s. Abbildung 7). Danach folgen der Erhalt und die Begründung einer standorttypischen Baumartenzusammensetzung, der Erhalt und die Schaffung von Totholz sowie die aktive Waldrandgestaltung.

Eine geringe Bedeutung beim Waldnaturschutz gegen Entgelt haben die Schaffung lichter Wälder, der Erhalt historischer Waldnutzungsformen und beispielsweise die Vernetzung von Biotopen.

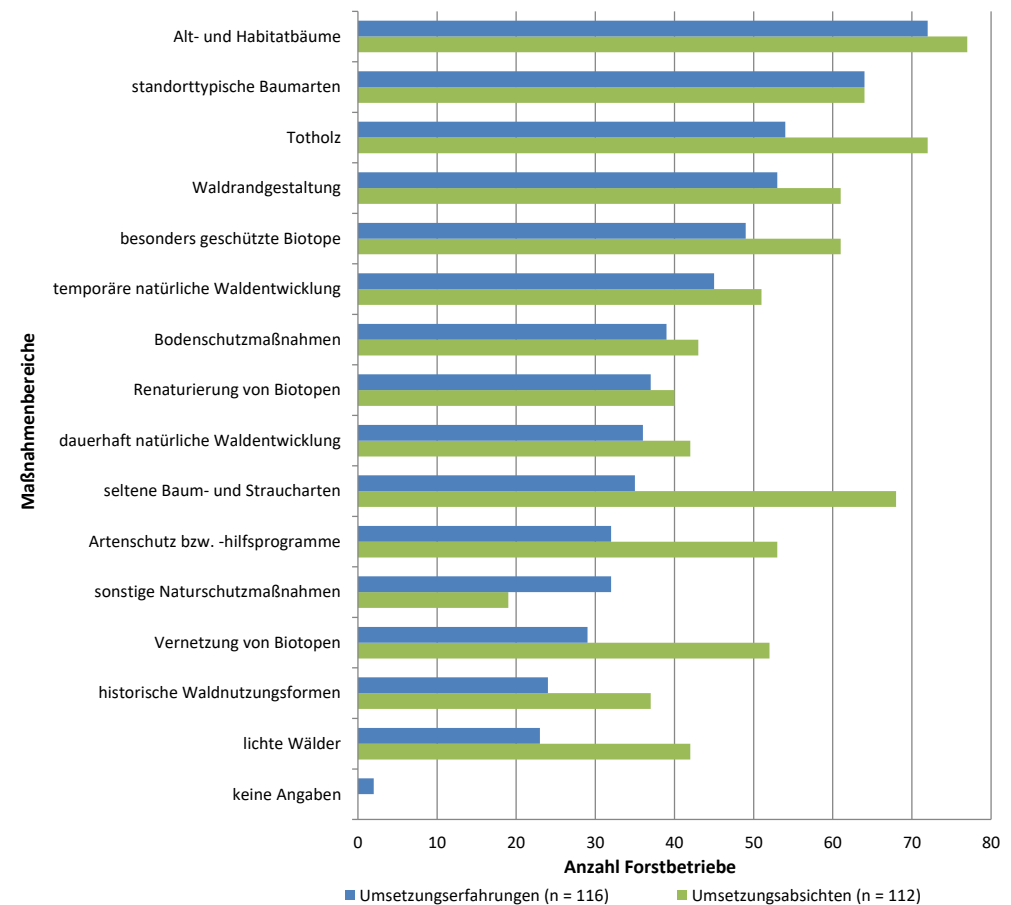

Abbildung 7: Realisierte und beabsichtigte Waldnaturschutzmaßnahmen gegen Entgelt (Mehrfachnennungen möglich, N=195), Quelle: Kownatzki et al. 2017 
Die mittels Waldnaturschutz gegen Entgelt jährlich vereinbarte Maßnahmenflächen der Betriebe liegt überwiegend unterhalb von 10 Hektar (s. Abbildung 8), unabhängig von der Größe der befragten Betriebe.

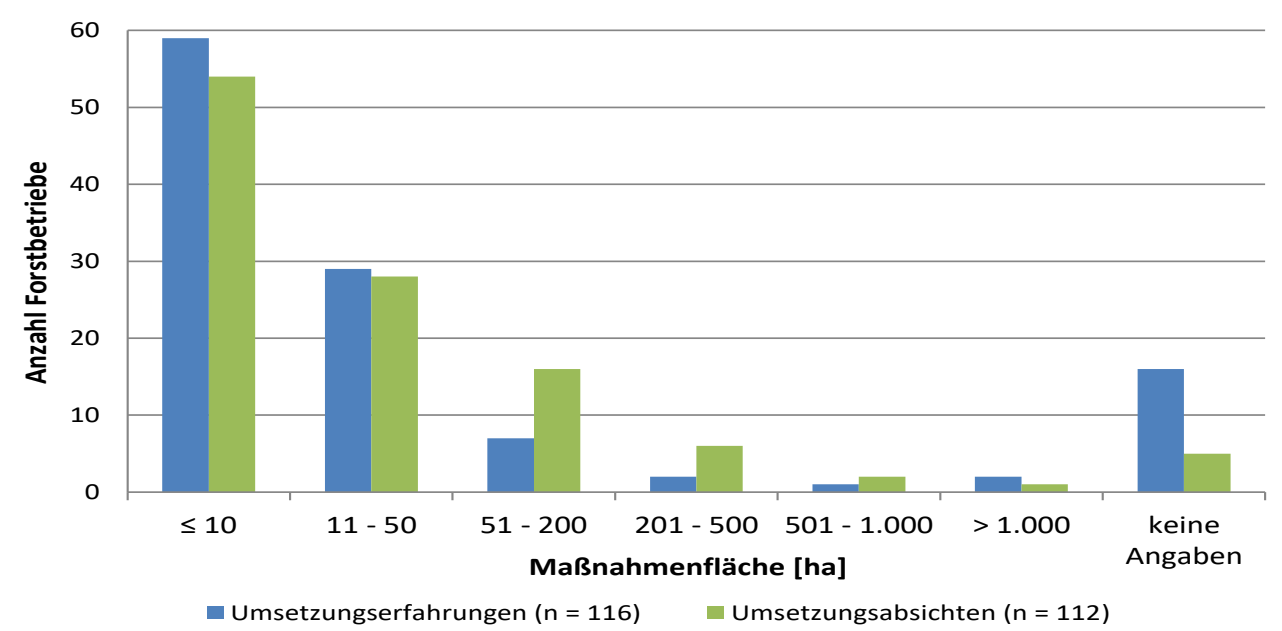

Abbildung 8: Realisierte und beabsichtigte jährliche Maßnahmenfläche (Mehrfachnennungen möglich, N=195), Quelle: Kownatzki et al. 2017

\subsection{Potenziale \& Hemmnisse sowie Identifizierung von Erfolgsfaktoren}

Der Stand der Forschung und die Ergebnisse der Onlinebefragung im Rahmen des WaVerNaProjekts zeigen auf, dass der Vertragsnaturschutz im Wald eine Vielzahl von Potenzialen bietet, gleichzeitig aber mit großen und teilweise langfristig bestehenden Hemmnissen versehen ist.

Das Ziel des nächsten Schritts im WaVerNa-Verbundprojekts war die Analyse von Fallbeispielsbetrieben zur Identifikation dieser Potenziale und Hemmnisse, um daraus erfolgsrelevante Gestaltungsfaktoren für Vertragsnaturschutzmaßnahmen im Wald aus Sicht von Privatforstbetrieben ableiten zu können.

\subsubsection{Methodik}

Zur Erhebung und Identifikation der für Vertragsnaturschutz im Wald relevante Erfolgsfaktoren, wurden in verschiedenen Bundesländern Fallbeispielsbetriebe analysiert. Die Analyse der Fallbeispielsbetriebe erfolgte im persönlichen Gespräch mittels leitfadengestützter Experteninterviews. Das leitfadengestützte Experteninterview ist im Gegensatz zu der offenen Onlinebefragung aus Kapitel 2.3.2 den qualitativen Methoden der Sozialforschung zuzurechnen, da nur ein vergleichsweise kleiner Personenkreis befragt wird (Mieg und Näf 2006). Zentraler Bestandteil der qualitativ-orientierten Methode sind die Offenheit, die Reflexivität, die Kommunikation sowie die Problemorientierung der Befragten (Atteslander 2010). 
Das Experteninterview ist ein Instrument der Forschung und verfolgt sachliche Ziele. Die Vorgehensweise der Beteiligten ist konstruktiv, die Motivation des Befragten ist die Vermittlung von Wissen und Können (Mieg und Näf 2006). Das leitfadengestützte Experteninterview ist den teilstrukturierten Interviewsituationen zuzurechnen, bei denen ein im Vorfeld formulierter Fragebogen als Grundlage dient, die Reihenfolge der Fragen jedoch individuell anpassbar ist (Schnell et al. 2013).

\subsubsection{Leitfaden}

Für das Interview war es notwendig, einen Leitfaden vorab zu erstellen, der den Gesprächspartnern dient, die Fragen vollständig und hinreichend spezifisch zu behandeln (Mieg und Näf 2006). Gleichzeitig verhindert die intensive Auseinandersetzung mit dem Thema bei der Erstellung des Leitfadens, dass sich der Forscher als inkompetenter Gesprächspartner erweist (Meuser und Nagel 1991). Der Leitfaden folgt dem empfohlenen Schema der Fachliteratur mit Einstiegsfragen, Hauptteil zum Themenschwerpunkt und Abschlussfragen mit Aus- und Rückblick (Mieg und Näf 2006). Aus Übersichtlichkeitsgründen ist der Fragebogen tabellarisch aufgebaut (Krüger et al. 2014).

Im ersten Block des gemeinsamen Fragebogens aller Teilprojekte wurden allgemeine Fragen zur antwortenden Person sowie zum vertretenden Betrieb gestellt. Besonderes Augenmerk lag dabei auf der Baumartenzusammensetzung, dem Anteil von Flächen mit Naturschutzauflagen, der Personalstruktur sowie dem Hauptziel des Betriebes.

Im zweiten Teil des Fragebogens, dem Hauptteil, wurde abgefragt, welche Naturschutzmaßnahmen gegen Entgelt in der Vergangenheit und gegenwärtig durchgeführt wurden. Diese wurden hinsichtlich der Vertragspartner, der Ausgestaltung, der Finanzierung sowie des Umfangs eingehend analysiert. Hinzu kam die Erhebung des Zeitaufwands der Mitarbeiter für die umgesetzten Maßnahmen (Auswertung s. Kapitel 2.5).

Im dritten Block wurden die Interviewpartner hinsichtlich ihrer Erfahrungen, den entstandenen Problemen und Konflikten sowie der Angemessenheit des gezahlten Entgelts befragt. Weiterhin wurden die Gesprächspartner um Einschätzungen zu den Risiken aus betrieblicher Sicht gebeten. Hinzu kam eine Überprüfung der ökologischen Wirksamkeit der umgesetzten Maßnahmen aus Sicht der Betriebe, ergänzt um Fragen zu etwaigen Kontrollen durch Vertragspartner oder Dritte.

Im letzten Block wurden die Gesprächspartner um Einschätzungen gebeten, welche rechtlichen Risiken aus ihrer Sicht bei der Teilnahme an Naturschutzmaßnahmen gegen Entgelt entstehen 
und im Hinblick auf die Zukunft, welche Chancen sie in einem solchen Instrument sehen. Abschließend bestand für alle befragten Experten die Gelegenheit noch weitere, im Fragebogen nicht berücksichtigte, Aspekte anzuführen. Vielfach wurden solche Punkte ergänzend zu den Fragen im Gespräch vorab schon geäußert, so dass sie bereits erfasst waren.

\subsubsection{Auswertung der Experteninterviews/Fallstudien}

Die Fallstudien dienen, aufbauend auf der offenen Onlinebefragung, der vertiefenden Analyse von Sachverhalten, Potentialen und Hemmnissen in der Umsetzung von Vertragsnaturschutzmaßnahmen im Wald. Sie verfolgen nicht den Zweck statistisch abgesicherter Ergebnisse. Auf qualitativer Basis sollen Kernelemente identifiziert werden, die für Gelingen oder Scheitern von Naturschutzmaßnahmen gegen Entgelt im Wald von entscheidender Bedeutung sind. Die Auswertung der Interviews zielt mittels Vergleich der Antworten auf eine Erarbeitung der überindividuell-gemeinsamen Wissensbestände der Befragten hin (Gläser und Laudel 2010).

\subsubsection{Auswahl der Betriebe}

Die Gewinnung der Fallbeispielsbetriebe folgte aus der in Kapitel 2.3 beschriebenen offenen Onlinebefragung, bei der Teilnehmer sich bereiterklären konnten, für eine tiefergehende Analyse zur Verfügung zu stehen. 35 Betriebe waren nach eigener Aussage hierzu bereit. Daraus konnten schließlich 20 Betriebe gewonnen werden. Ergänzt wurden sie um zwei weitere Betriebe, die zur Abdeckung fehlender Bundesländer zusätzlich von den Projektpartnern angesprochen wurden. Somit belief sich die Gesamtzahl der Fallbeispielsbetriebe auf 22 in insgesamt 8 Bundesländern (Baden-Württemberg, Bayern, Brandenburg, Hessen, Mecklenburg-Vorpommern, Niedersachsen, Nordrhein-Westfalen und Thüringen). Die Stadtstaaten wurden aufgrund der geringen Flächenausstattung nicht berücksichtigt. Für die Bundesländer Rheinland-Pfalz, Saarland, SachsenAnhalt, Sachsen und Schleswig-Holstein lagen entweder keine Informationen über Vertragsnaturschutzprogramme im Wald vor oder es standen keine Betriebe für eine Fallbeispielsanalyse zur Verfügung.

\subsubsection{Ansprechpartner \& Experten}

Die Befragten innerhalb der Forstbetriebe waren entweder die Eigentümer oder Mitarbeiter in leitenden Funktionen (Betriebsleiter/Revierleiter). Diese Personen können als Experten eingestuft werden, da sie aufgrund ihrer beruflichen Tätigkeit über die notwendige Erfahrung und das spezifische Wissen zu Planung und Durchführung von Vertragsnaturschutzmaßnahmen verfü- 
gen (Mieg und Näf 2006). Die Interviewpartner erhielten vorab den Fragebogen, um die notwendigen Unterlagen und Daten vorbereiten zu können. Die Interviews wurden im persönlichen Kontakt in den Forstbetrieben geführt, anschließend erfolgte ein Waldbegang, um die durchgeführten Maßnahmen fotografisch dokumentieren zu können. Dieses kombinierte Verfahren (Vorabübersendung \& persönliches Gespräch) erhält die relativ höhere Reaktivität des direkten Gesprächs und ermöglicht die Erweiterung um die Verwendung komplexer Fragestellungen (Atteslander 2010). Ein solches Interview ist geeignet, betriebliches Wissen zu rekonstruieren, das gleichzeitig durch die im Leitfaden enthaltenen Themenbereiche strukturiert wird (Krüger et al. 2014). Die Interviews wurden zwischen Dezember 2016 und Dezember 2017 durchgeführt.

\subsubsection{Ergebnisse:}

\subsubsection{Potenziale und Hemmnisse}

Ein Ziel der Fallbeispielsanalysen war die Identifikation der Potenziale und Hemmnisse von Vertragsnaturschutzmaßnahmen im Wald in Betrieben, die in der Vergangenheit und gegenwärtig solche Maßnahmen umgesetzt haben oder umsetzen und daher über einen ausreichenden Erfahrungsschatz verfügen.

Im WaVerNa-Verbundforschungsvorhaben haben alle vier Teilprojekte (ökologische Analysen, ökonomische Analysen Angebots- \& Nachfrageseite, rechtliche Analysen) die aus Ihrer Sicht relevanten Potenziale und Hemmnisse analysiert und an geeigneter Stelle veröffentlicht (s. Seintsch et al. 2018). Die nachfolgend dargestellten Ergebnisse beziehen sich aufgrund der Ausrichtung dieser Arbeit ausschließlich auf die Sicht der Forstbetriebe, die im Projekt als „Anbieter“ von Naturschutzleistungen, bzw. geeigneten Waldflächen bezeichnet wurden.

Aus den Antworten der befragten Experten lassen sich die in Tabelle 1 dargestellten Potenziale zusammenfassen. Die häufigste Nennung erfuhr dabei die erwartete Akzeptanzsteigerung der Waldbewirtschaftung beim Vertragspartner (Naturschutz), aber auch eine Imageverbesserung in der Bevölkerung sowie der Politik. Unmittelbar damit im Zusammenhang stehend wurde auch die Hoffnung geäußert, mittels freiwilliger Naturschutzmaßnahmen ordnungsrechtliche Vorgaben zu vermeiden. Abschließend bestand unter den Befragten auch die Erwartung, Naturschutzmaßnahmen in oder außerhalb von Schutzgebieten über freiwilliges Engagement angemessen vergütet zu bekommen. 
Tabelle 1: Potenziale und Hemmnisse von Naturschutzmaßnahmen gegen Entgelt im Wald aus Sicht der befragten Fallbeispielsbetriebe des WaVerNa-Projekts inklusive Handlungsempfehlungen, Zusammenfassung, Quelle: eigene Darstellung

\begin{tabular}{lll}
\hline & Potenziale & Hemmnisse \\
\hline Antworten der & - Akzeptanzsteigerung & - Langfristige Finanzierung unsicher (Staat \\
Fallbeispielsbetriebe & - Vergütung von Naturschutzmaßnahmen & unzuverlässiger Vertragspartner) \\
& - In Schutzgebieten: Vergütung von & - Transaktionskosten hoch \\
& betrieblichen Einschränkungen bzw. & - Gebietskulisse z.T. auf Schutzgebiete \\
& - Imageförderung bei Öffentlichkeitsarbeit & beschränkt \\
& - Vermeidung von hoheitlichen Regelungen & - \\
& & Technische Probleme bei GIS-Einmessung \\
& & und Datenaustausch \\
\hline Handlungsempfehlungen & - Effiziente Lösungen sind zu entwickeln/umzusetzen \\
& - Langfristige Finanzierung durch alternative Modelle sichern (Landes-, bzw. Bundesstiftung) \\
& - Ausweitung der Programme und Angebote auf die Normallandschaft \\
\hline
\end{tabular}

Gegenüber den häufig eher als vage wahrgenommenen Potenzialen äußerten sich die befragten Experten zu einer Reihe von konkreten Hemmnissen, die aktuell oder in der Vergangenheit zu Problemen bzw. der Beendigung von Naturschutzmaßnahmen gegen Entgelt im Wald geführt haben.

Vorrangiges Hemmnis, dass von nahezu allen Fallbeispielsbetrieben geäußert wurde, war der Mangel einer langfristigen Finanzierung der Maßnahmen. Der Staat als Vertragspartner unterliegt regelmäßig dem Haushaltsvorbehalt von Finanzmitteln, so dass diese meist nur einjährig und sehr selten länger als für fünf Jahre sicher bereitgestellt werden können. Eine solch kurzfristige Finanzierung steht im Widerspruch zu den langfristigen Zweckbindungszeiträumen (bis zu 20 Jahre) der angebotenen Maßnahmen. Ergänzend wurde in diesen Fällen die angebotene Vergütung nicht als ausreichend wahrgenommen, wohingegen sie bei Maßnahmen gleicher Finanzierungs- und Zweckbindungszeiträume (bspw. das Vorhalten von Totholz) als akzeptabel empfunden wurde.

Einige der Fallbeispielsbetriebe, die über ökologisch hochwertige Flächen außerhalb von Schutzgebieten verfügen, konnten in der Vergangenheit nicht an freiwilligen Naturschutzmaßnahmen teilnehmen, da diese stets auf Flächen mit Schutzgebietsstatus (NSG, LSG, Natura 2000) beschränkt waren. Die, auf geldgebender Seite aus Allokationsgründen nachvollziehbare Fokussierung auf Flächen in Schutzgebieten, erschwert den Willigen die Umsetzung von Naturschutzmaßnahmen gegen Entgelt.

Abschließend stellen technische Probleme bei der Umsetzung der Maßnahmen und dem Datenaustausch zur Antragstellung und Kontrolle zwischen den Vertragspartnern noch ein großes Hemmnis dar, das sich in vielen Betrieben allerdings erst nach Beginn der Maßnahmen gezeigt 
hat und nicht im Vorfeld abschätzbar war. Da kein allgemeiner Datenstandard für die Einmessung und Weiterverarbeitung von Geoinformationen vorlag, kam es in vielen Betrieben immer wieder zu Problemen.

\subsubsection{Erfolgsfaktoren}

Aus den 22 Fallbeispielsanalysen des WaVerNa-Projekts wurden vier maßgebliche Erfolgsfaktoren identifiziert (Franz et al. 2018a), deren beiderseitig zufriedenstellende Ausgestaltung vorhanden sein muss, um den Vertragsnaturschutz im Wald zu einem erfolgreichen Abschluss zu führen:

\subsection{Kontinuität}

Die Kontinuität von Vertragsnaturschutzmaßnahmen umfasst eine langfristige Ausgestaltung sowohl der Maßnahmen als der Finanzierungen. Im besten Falle finden die forstlichen Planungszeiträume Berücksichtigung (Forsteinrichtungszeitraum: 10 Jahre). Zur Steigerung der Akzeptanz kann zwischen Programm- und Vertragslaufzeit unterschieden werden. Innerhalb eines Programms können Betriebe mehrfach Folgeverträge abschließen, um bei gewisser Flexibilität die Kontinuität der ökologischen Wirksamkeit zu gewährleisten (Franz et al. 2018a). Eine praktikable Vertragsklausel zur Sicherung der Kontinuität könnte auch darin bestehen, dass sich die Vertragsdauer jeweils automatisch verlängert, wenn keine der Vertragsparteien zum Ende der Vertragslaufzeit die Beendigung erklärt. Um die Kontinuität der Finanzierung sicherzustellen, wäre eine Abkehr von Finanzmitteln unter Haushaltsvorbehalt notwendig, indem die Maßnahmengelder bspw. aus Stiftungserträgen zur Verfügung gestellt werden.

\subsection{Flexibilität}

Die Flexibilität beschreibt die Anpassungsmöglichkeit von Naturschutzmaßnahmen an örtliche Gegebenheiten, betriebliche Besonderheiten oder sonstige Abläufe. Sie ist räumlich, zeitlich und sachlich zu verstehen. Die räumliche Flexibilität ist notwendig, um bspw. auch außerhalb von Schutzgebietskulissen Verträge abschließen zu können. Auf sachlicher Ebene sind die bestehenden Verträge oftmals zu aufwändig und führen zu einem unverhältnismäßigen Verwaltungsaufwand. Notwendig wären simplere Lösungen oder Rahmenverträge, die leicht anpassbar sind. Aus zeitlicher Sicht müssen die Antragsfristen und Laufzeiten angemessen an die forstbetrieblichen Abläufe angepasst werden. Lange Zweckbindungsfristen ohne angemessene finanzielle Kompensation wirken hemmend. 


\subsection{Fairness}

Unter Fairness wird im Zusammenhang mit Vertragsnaturschutzmaßnahmen das „anständige, gerechte und ehrliche Verhalten aller Beteiligten“ verstanden (Franz et al. 2018a). Das daraus resultierende Vertrauen bildet die Grundlage für erfolgreiche Verträge. In den Fallbeispielsbetrieben war oftmals eine Diskrepanz zwischen den Vertragspartnern erkennbar, es fanden keine Verhandlungen auf Augenhöhe statt, der Waldbesitzer empfand seine Rolle oftmals als die des Bittstellers. Ein solches Ober-Unterordnungsverhältnis konterkariert die Idee eines freiwilligen Vertragsnaturschutzes. Ordnungsrechtliche Festsetzungen von Naturschutzauflagen in Natura 2000-Gebieten, deren Umsetzung zunächst noch mittels Vertragsnaturschutz in Aussicht gestellt wurde, haben auf der waldbesitzenden Seite zu einem grundsätzlichen Misstrauen gegenüber dem amtlichen Naturschutz geführt. Es bleibt für die Waldbesitzer weithin unverständlich, weshalb die aus ihrer Bewirtschaftung resultierenden, ökologisch wertvollen Zustände durch Untersagen dieser Bewirtschaftung gerettet werden müssten. Ordnungsrechtliche Auflagen im Kontext mit Natura 2000 werden als Bestrafung vorbildlicher Forstwirtschaft verstanden.

Weiterhin wird unter Fairness die Angemessenheit des Entgelts verstanden. In bestehenden Förderprogrammen decken die gezahlten Beträge oftmals nur die reinen Mindererträge ab, ohne den Verwaltungsmehraufwand angemessen zu berücksichtigen oder gar eine „Gewinnperspektive“ zu eröffnen. Anreizkomponenten zur Teilnahme und etwaige Risikozuschläge finden ebenfalls wenig Anwendung.

\subsection{Rechtssicherheit}

Der Erfolgsfaktor Rechtssicherheit umfasst die Klarheit, Beständigkeit und Nachvollziehbarkeit der Rechtsgrundlagen und die Transparenz der mit Vertragsnaturschutz einhergehenden Risiken (Franz et al. 2018a). Förderrichtlinien besitzen einen hohen Komplexitätsgrad, der für Forstpraktiker schwer nachvollziehbar ist. Unklare Regelungen hinsichtlich des Monitorings und Berichtswesens schaffen zusätzliche Rechtsunsicherheiten für beide Vertragsparteien.

Besondere Bedeutung für alle Beteiligten im Vertragsnaturschutz besitzt das sog. Wiederaufnahmeprivileg nach $\S 30$ BNatschG, also die gesetzlich garantierte Zulässigkeit der Rückkehr zur regulären, ordnungsgemäßen Forstwirtschaft nach Ablauf eines vertraglichen Zweckbindungszeitraums. Diese steht jedoch möglicherweise im Wiederspruch zu artenschutzrechtlichen Regelungen des $\S 44$ BNatschG, der greift, wenn sich eine besonders geschützte Tier- oder Pflanzenart in einem Gebiet etabliert hat. Diese ist grundsätzlich durch Unterlassen gefährdender 
Handlungen zu schützen. Im Vertragsnaturschutz, der unter anderem auch die Förderung geschützter Arten anstrebt, führen die widerstreitenden Regelungen zu großer Unsicherheit. Hier bedarf es in Zukunft einer Rechtssicherheit gewährleistenden Regelung.

Für gemischte Betriebe der Land- und Forstwirtschaft ist auch das Risiko von sog. Cross-Compliance-Verstößen von hoher Bedeutung. Werden während einer Kontrolle Verstöße gegen die vertraglichen Auflagen im Wald festgestellt, drohen Kürzungen der landwirtschaftlichen Direktzahlungen. Ein solches Risiko lässt Vertragsnaturschutzmaßnahmen im Wald für gemischte Betriebe schnell unattraktiv werden und erfordert eine entsprechende Berücksichtigung in der Ausgestaltung der Maßnahmenangebote.

\subsubsection{Handlungsempfehlungen \& Lösungsansätze}

Aus der Erhebung der Potenziale und Hemmnisse ließen sich bereits Handlungsempfehlungen für zukünftige Ausgestaltung ableiten (s. Tabelle 1). Insbesondere die Sicherung einer langfristigen Finanzierung der Maßnahmen sowie die Ausweitung von Vertragsnaturschutzmaßnahmen auf die Normallandschaft außerhalb von Schutzgebieten können die vorhandenen Potenziale besser nutzbar machen.

Für die vorgenannten Erfolgsfaktoren wurden im WaVerNa-Projekt Lösungsansätze entwickelt, um für zukünftige Vertragsnaturschutzmaßnahmen im Wald eine erfolgreichere Umsetzung zu ermöglichen (s. Abbildung 9).

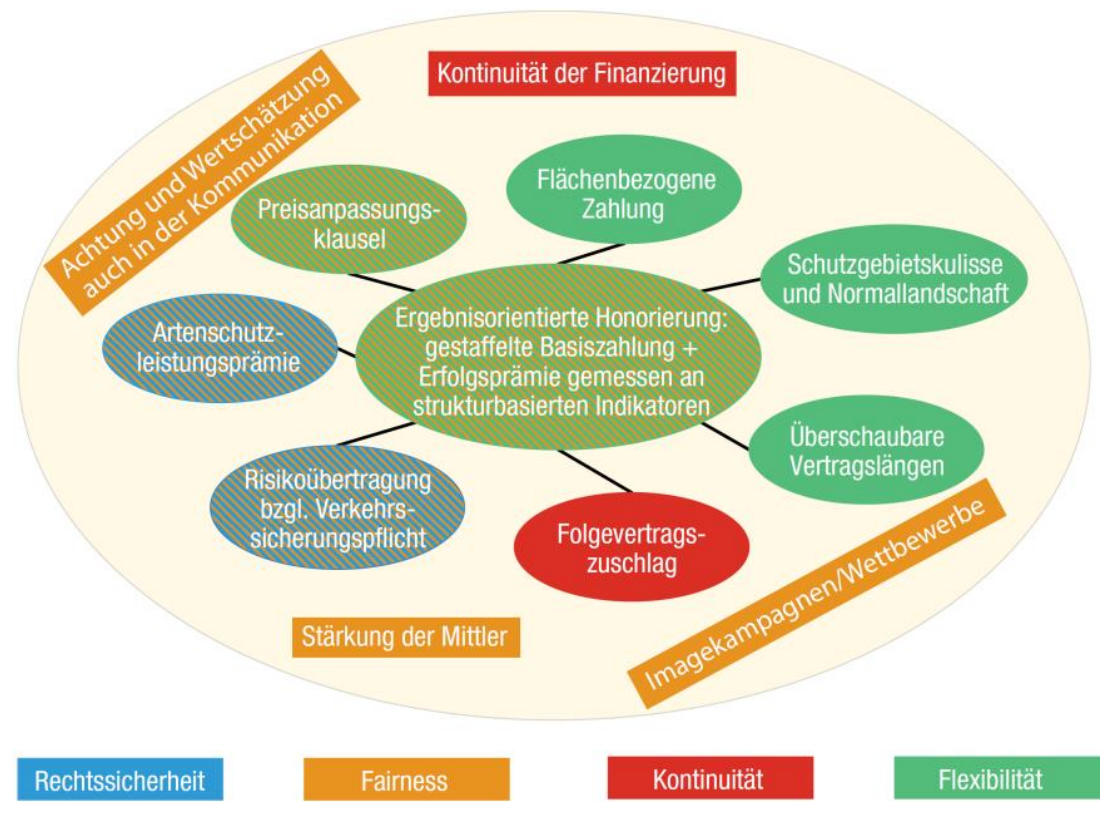

Abbildung 9: Schematische Darstellung für Lösungsansätze innerhalb der identifizierten Erfolgsfaktoren, Quelle: Franz et al. $2018 a$ 
In Abbildung 9 sind die Lösungsvorschläge zugeordnet zu Erfolgsfaktoren schematisch dargestellt. Im äußeren Bereich sind die Rahmenbedingungen in eckigen Feldern dargestellt, die unabdingbar für eine erfolgreiche Umsetzung sind. Hierzu zählen die Kontinuität der Finanzierung, die gegenseitige Achtung und Wertschätzung als Gesprächs- und Verhandlungspartner auf Augenhöhe, die Stärkung von Mittlern zwischen Naturschutz und Waldeigentümern sowie Imagekampagnen zur Verbesserung der öffentlichen Wahrnehmung solcher freiwilliger Naturschutzmaßnahmen.

Als zentrales Lösungselement wurde im WaVerNa-Projekt die Umgestaltung der Naturschutzmaßnahmen weg von handlungsorientierten (aktiver Waldumbau mit klaren Vorgaben zu Pflanzenzahlen und Baumarten) hin zu einer ergebnisorientierten Honorierung vorgeschlagen. Die vorgeschlagene Honorierung basiert auf einer gestaffelten Basiszahlung zuzüglich Erfolgsprämien in Abhängigkeit von strukturbasierten Indikatoren. Der Waldbesitzer erhält somit die notwendige Flexibilität, um unter Berücksichtigung der natürlichen und betrieblichen Besonderheiten verschiedene Ergebnisstufen zu erreichen. Zusätzliche Lösungselemente, die von Franz et al. (2018a) vorgeschlagen werden, sind in der Grafik in den Ovalen um den Mittelpunkt aufgeführt.

\subsection{Auswirkungen von Naturschutzmaßnahmen auf Verwaltungs- \& Kon- trollkosten}

Neben den Ertragsveränderungen der forstlichen Bewirtschaftung durch alternative Baumarten oder ökologisch vorteilhaftere Behandlungskonzepte, wirken sich Naturschutzmaßnahmen im Wald auch auf der betrieblichen Managementebene aus. Es ist davon auszugehen, dass den Forstbetrieben durch ein Abweichen von der bisherigen, ökonomisch ausgerichteten Bewirtschaftung höhere Kontroll- und Verwaltungsaufwendungen entstehen (vgl. Sotirov 2017, S. 107). Im Rahmen der vorliegenden Arbeit sollen nachfolgend am Beispiel des Erhalts von Altund Habitatbäumen als häufigster Maßnahme der Naturschutzmaßnahmen gegen Entgelt im Wald die betrieblichen Erschwernisse beschrieben und quantifiziert werden.

\subsubsection{Ziel}

Im WaVerNa-Projekt wurde deutlich, dass nicht ausschließlich der Holzwert Einfluss auf die Entscheidung zur Teilnahme an einer Vertragsnaturschutzmaßnahme hat, sondern auch eine Integrierbarkeit in forstliche Organisations- und Arbeitsabläufe gegeben sein muss. Ziel war es daher, die unmittelbaren und mittelbaren Auswirkungen der Umsetzung von Naturschutzmaßnahmen auf die Betriebsabläufe in den Betrieben zu erfassen und zu quantifizieren. 


\subsubsection{Methodik}

Anhand der im forstlichen Vertragsnaturschutz häufigsten Maßnahme, des Erhalts von Alt- und Habitatbäumen wurde der Verwaltungs- und Kontrollmehraufwand der Fallbeispielsbetriebe des WaVerNa-Verbundvorhabens analysiert. In einem Fragenblock des leitfadengestützten Interviews wurden die Gesprächspartner (Betriebs- oder Revierleiter) zum zeitlichen Aufwand für die verschiedenen Phasen der Planung, Umsetzung und Kontrolle von Naturschutzmaßnahmen gegen Entgelt im Wald befragt. Auf Basis einer Zeiterfassungstabelle wurden die Maßnahmen, unterteilt nach den Phasen des kybernetischen Regelkreises (Planung, Entscheidung, Durchführung, Kontrolle) aufgeteilt, abgefragt. Die Zeiterfassungstabelle wurde den Interviewpartner vorab zur Vorbereitung zur Verfügung gestellt.

Die bei den Interviewpartnern der Fallbeispielsbetriebe erhobenen Zeiten dienten maßgeblich der Zeitbedarfsermittlung pro ausgewiesenem Habitatbaum. Sie wurden in einem zweiten Schritt mit dem durchschnittlichen Personalaufwand pro Stunde einer Revierleitungskraft bewertet, so dass die verursachten Kosten ermittelt werden konnten. Diese fanden unter anderem als Datenbasis in der Fallstudie Hämeler Wald Verwendung (s. Kapitel 4.4).

Neben dem reinen Zeitbedarf werden zu einem späteren Zeitpunkt auch die Auswirkungen auf die Bewirtschaftung des verbleibenden Bestands unter Berücksichtigung der Durchführung von Naturschutzmaßnahmen gegen Entgelt anhand von Beispielbeständen untersucht. Mittels Stammverteilungsplänen und Kronenschirmflächen von ausgewählten, ökologisch wertvollen Bäumen wurden Gefährdungsbereiche und im Falle der Bestandesverjüngung die für die Nachfolgegeneration verhinderte Produktionsfläche ermittelt (s. Kapitel 4.5.).

\subsubsection{Ergebnisse}

\subsubsection{Zeiterfassungssysteme in den Fallbeispielsbetrieben}

Während der Interviews zeigte sich, dass die meisten befragten Forstbetriebe nicht über ein Zeiterfassungssystem für Revierleitungspersonal verfügen. Sowohl die Betriebs- als auch die Revierleiter werden fest vergütet und arbeiten in Vertrauensarbeitszeit. Eine Erfassung der geleisteten Stunden ist daher aus betrieblicher Sicht im Verhältnis zum entstehenden Aufwand nicht notwendig, da für gewöhnlich auch geleistete Überstunden nicht vergütet werden. Wenn dennoch eine Gesamtarbeitszeit erfasst wird, so wird diese in den seltensten Fällen auf einzelne Projekte oder Arbeitsbereiche aufgeteilt. In nur zwei der 24 Fallbeispielsbetrieben bestand ein System zur Zeiterfassung aller Mitarbeiter. 
Für Lohnempfänger (Waldarbeiter, Forstwirte) stellte sich die Situation vielfach anders dar. Diese sind zumeist verpflichtet, Stundennachweise zu führen, um somit dem Vorgesetzten ein Kontrollinstrument bereitzustellen. Der präzise Stundenaufschrieb ist auch noch ein Rudiment der Akkordverlohnung im Holzeinschlag, als der nachweisbare Holzeinschlag Grundlage der Lohnzahlung war (vgl. Minister für Umwelt, Raumordnung und Landwirtschaft NordrheinWestfalen 1987). In den Betrieben mit eigenen Forstwirten dienen die Stundenerfassungen nach Tätigkeitsbereichen teilweise noch zur Ermittlung von Lohnzusätzen, wie z.B. der Motorsägenentschädigung.

Für die Erfassung der in den Phasen einer Naturschutzmaßnahme gegen Entgelt im Wald geleisteten Stunden im Leitungsbereich, musste mangels Stundennachweise demnach vielerorts auf Einschätzungen der Interviewpartner zurückgegriffen werden.

\subsubsection{Zeitbedarf Alt- und Habitatbaumausweisung}

Die Maßnahme Alt- und Habitatbaumausweisung war nicht nur in der Onlinebefragung von Forstbetrieben die häufigste Naturschutzmaßnahme gegen Entgelt, auch in den Interviews mit den Fallbeispielsbetrieben wurde sie am häufigsten umgesetzt. Die konkrete Umsetzung erfolgte dabei mit einer Ausnahme stets durch die Teilnahme an Förderprogrammen der öffentlichen Hand. Die Mittelherkunft variierte je nach Finanzierungsart des Förderprogramms in den einzelnen Bundesländern (s. Kapitel 2.2).

Die von den Betrieben im Vorfeld durchzuführenden Arbeiten und die zum Nachweis der Bäume verwendeten Lokalisierungstechnologien (vornehmlich GNSS ${ }^{10}$-Handgeräte ohne Korrektursignale) unterschieden sich in der konkreten Ausgestaltung. In vielen Fallbeispielsbetrieben erfolgen die Phasen der Planung und Durchführung in einem Arbeitsschritt, da die notwendigen Planungsmaßnahmen für die Antragstellung untrennbar an die direkte Ausweisung und Markierung der Alt- und Habitatbäume geknüpft ist.

\footnotetext{
${ }^{10}$ GNSS (Globales Navigationssatellitensystem): Oberbegriff für Satellitengestützte Positionsbestimmung und Navigation. Hauptsächlich erfolgt die Lokalisation mittels des US-amerikanischen NAVSTAR GPS-Systems. Die Anwendung bspw. des europäischen Galileo-Systems oder des russischen GLONASS-Systems erfolgte in den Fallbeispielsbetrieben nicht.
} 
Tabelle 2: Zeitbedarf Umsetzung Naturschutzmaßnahme Alt- und Habitatbäume, Quelle: Fallbeispielsbetriebe WaVerNa-Projekt

\begin{tabular}{|c|c|c|c|c|c|c|c|c|c|c|}
\hline \multirow[t]{2}{*}{ Fallstudie } & \multirow{2}{*}{$\begin{array}{c}\text { Anzahl } \\
\text { Bäume } \\
\text { [St] }\end{array}$} & \multicolumn{3}{|c|}{ Zeitbedarf } & \multicolumn{5}{|c|}{ Je Baum } & \multirow{2}{*}{$\begin{array}{c}\text { Kosten pro } \\
\text { Baum } \\
\text { Pers.-Aufw.: } 60 \\
\text { } / \text { Std. brutto } \\
\\
\text { [€/St.] }\end{array}$} \\
\hline & & Planung & Durchführung & Kontrolle & $\begin{array}{c}\text { Planung \& } \\
\text { Durchführung } \\
\text { [h/St.] }\end{array}$ & $\begin{array}{c}\text { Kontrolle } \\
\text { [h/St.] }\end{array}$ & $\begin{array}{l}\text { Gesamt } \\
\text { [h/St.] }\end{array}$ & \begin{tabular}{|c|} 
Planung \& \\
Durchführung \\
relativ \\
{$[\%]$}
\end{tabular} & $\begin{array}{c}\text { Kontrolle } \\
\text { relativ } \\
\text { [\%] }\end{array}$ & \\
\hline Betrieb 1 & 300 & 20 & 80 & 32 & 0,3 & 0,1 & 0,4 & $76 \%$ & $24 \%$ & 26 \\
\hline Betrieb 2 & 40 & 4 & 8 & 4 & 0,3 & 0,1 & 0,4 & $75 \%$ & $25 \%$ & 24 \\
\hline Betrieb 3 & 130 & 12 & 32 & 6 & 0,3 & 0,05 & 0,4 & $88 \%$ & $12 \%$ & 23 \\
\hline Betrieb 4 & 105 & & 48 & 5 & 0,5 & 0,05 & 0,5 & $91 \%$ & $9 \%$ & 30 \\
\hline Betrieb 5 & 300 & & 56 & 40 & 0,2 & 0,1 & 0,3 & $58 \%$ & $42 \%$ & 19 \\
\hline Betrieb 6 & 99 & & 26 & 10 & 0,3 & 0,1 & 0,4 & $72 \%$ & $28 \%$ & 22 \\
\hline Betrieb 7 & 152 & & 38 & 15 & 0,3 & 0,1 & 0,3 & $72 \%$ & $28 \%$ & 21 \\
\hline \multicolumn{5}{|l|}{ Mittelwert } & 0,3 & 0,1 & 0,4 & $77 \%$ & $23 \%$ & 24 \\
\hline
\end{tabular}

Die befragten Fallbeispielsbetrieben haben zwischen 40 bis 300 Alt- und Habitatbäume im Rahmen der Naturschutzmaßnahmen gegen Entgelt ausgewiesen. Die Ausweisung erfolgte dabei einzeln, in Gruppen oder sogar bestandsweise. Aus dem angegebenen Zeitbedarf ergibt sich ein durchschnittlicher Zeitbedarf von 0,3 h/Baum für die Planungs- und Durchführungsphase (variiert zwischen 0,2 h/Baum bis 0,5 h/Baum), für nachfolgende Kontrollen werden im Mittel 0,1 h/Baum benötigt (0,05 h/Baum bis 0,1 h/Baum) (s. Tabelle 2). Daraus ergibt sich ein Verhältnis des Zeitbedarfs von 77 \% für die aktive Durchführung der Maßnahme zu 23 \% für vom Vertragspartner/Geldgeber eingeleitete Kontrollen. Bei einer Bewertung der Arbeitsstunde mit $60 € / \mathrm{h}$ brutto, ergeben sich daraus durchschnittliche Personalkosten der gesamten Naturschutzmaßnahme von $24 € /$ Baum.

Auffällig dabei war, dass die Anzahl der ausgewiesenen Alt- und Habitatbäume keinen nachweisbaren Einfluss auf den Zeitbedarf je Baum hat. Es ist in den befragten Betrieben demnach kein positiver Skaleneffekt durch eine höhere Anzahl von ausgewiesenen Bäumen erkennbar.

Die Kontrolle der umgesetzten Maßnahme durch die Vertragspartner unterschied sich je nach Bundesland stark. Teilweise wurden diese ohne Beisein der Interviewpartner durchgeführt und verursachten nur vor- oder nachgelagerten administrativen Aufwand, teilweise war eine Anwesenheit vor Ort durch die Betriebs-/Revierleiter erforderlich. Dennoch ist der Zeitbedarf bezogen auf den Einzelbaum von den befragten Betrieben sehr ähnlich eingeschätzt worden. 


\subsection{Vertragsnaturschutz in der Landwirtschaft}

Im Folgenden Kapitel soll der aktuelle Stand und die Entwicklung des Vertragsnaturschutzes in der Landwirtschaft auf Basis vorhandener Literatur hinsichtlich seiner rechtlichen Grundlagen, der Finanzierungsstruktur sowie der erfolgswirksamen Gestaltungsaspekte zusammenfassend analysiert und dokumentiert werden. Hier ergeben sich Anknüpfungspunkte an die vom Autor verfasste Masterarbeit zur Analyse von Zahlungen für Agrarumweltmaßnahmen (Blomberg 2015).

\subsubsection{Rechtliche Grundlage \& Finanzielle Ausstattung}

Vertragsnaturschutz in der Landwirtschaft, maßgeblich umgesetzt durch das Instrument der Agrarumweltmaßnahmen ${ }^{11}$ (Niens und Marggraf 2010), gilt gemeinhin als Erfolgsmodell (Kownatzki et al. 2017). Die Ausgestaltung und Finanzierung von Vertragsnaturschutz in der Landwirtschaft basiert maßgeblich auf der zweiten Säule der Gemeinsamen Agrarpolitik (E$\mathrm{LER}^{12}$ ). Aus der Abbildung 10 ist die zeitliche Entwicklung der Zahlungen innerhalb der Europäischen Union und ihrer Vorläufer für die Gemeinsame Agrarpolitik erkennbar. Seit 2004 nehmen die Zahlungen für die ländliche Entwicklung, die sog. Zweite Säule rund $25 \%$ der Gesamtzahlungen ein. Aus diesen Mitteln stammen auch die Zahlungen für Agrarumweltmaßnahmen in der Landwirtschaft. Grundsätzlich sind darin auch die Zahlungen für Waldumwelt- und Klimaschutzmaßnahmen enthalten, so sie denn zur Anwendung kommen.

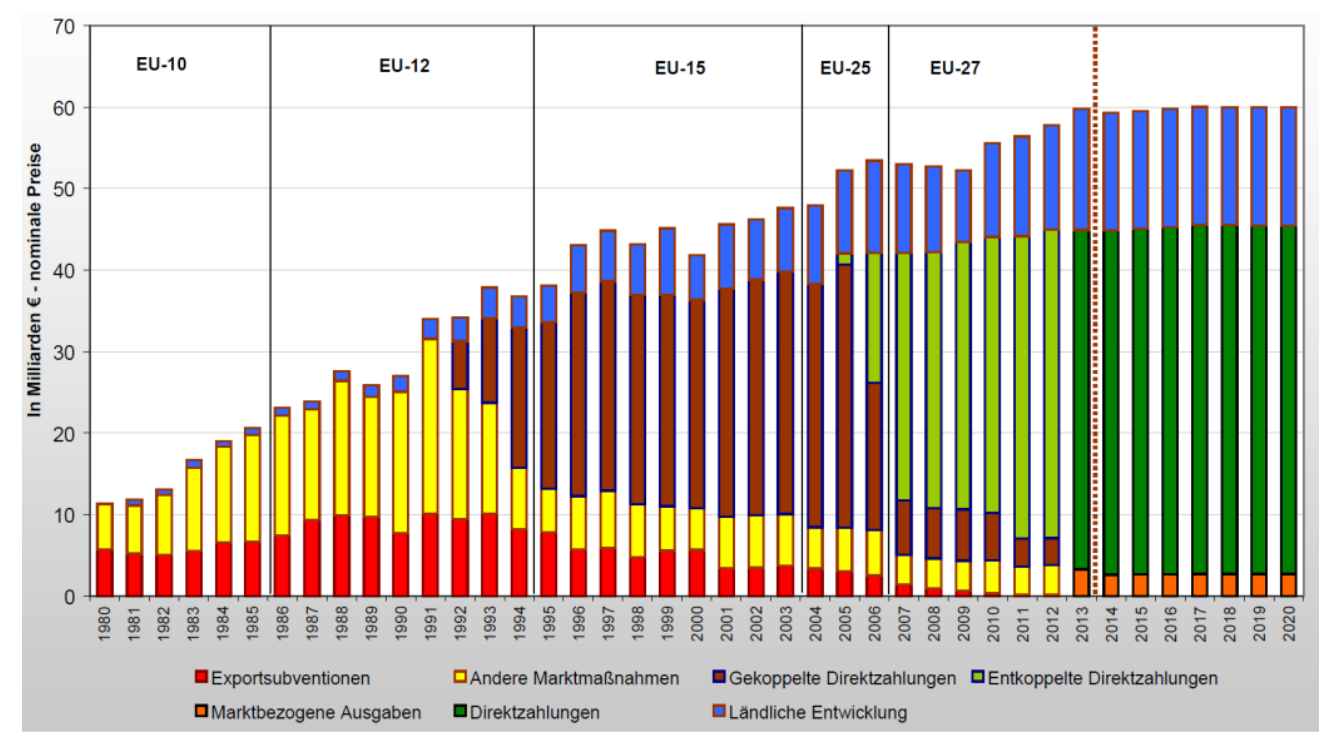

Abbildung 10: Entwicklung der GAP Zahlungen in Mrd. € von 1980 - 2020. Quelle: Europäische Kommission, GD Landwirtschaft und ländliche Entwicklung

\footnotetext{
${ }^{11}$ Alternativ auch Agrarumwelt- und Klimaschutzmaßnahmen genannt

12 Europäischer Landwirtschaftsfonds für die Entwicklung des ländlichen Raums
} 
Der Europäische Landwirtschaftsfonds für die Entwicklung des ländlichen Raums setzt sich in der aktuellen Förderperiode 2014 - 2020 aus sechs Unterzielen zusammen, die ergänzt werden um drei Querschnittsthemen. Auf europäischer Ebene ist er mit einem Finanzvolumen von 8,3 Mrd. € zuzüglich 1,1 Mrd. € aus der nationalen Umschichtung und der nationalen Mittel ausgestattet (s. Abbildung 11).

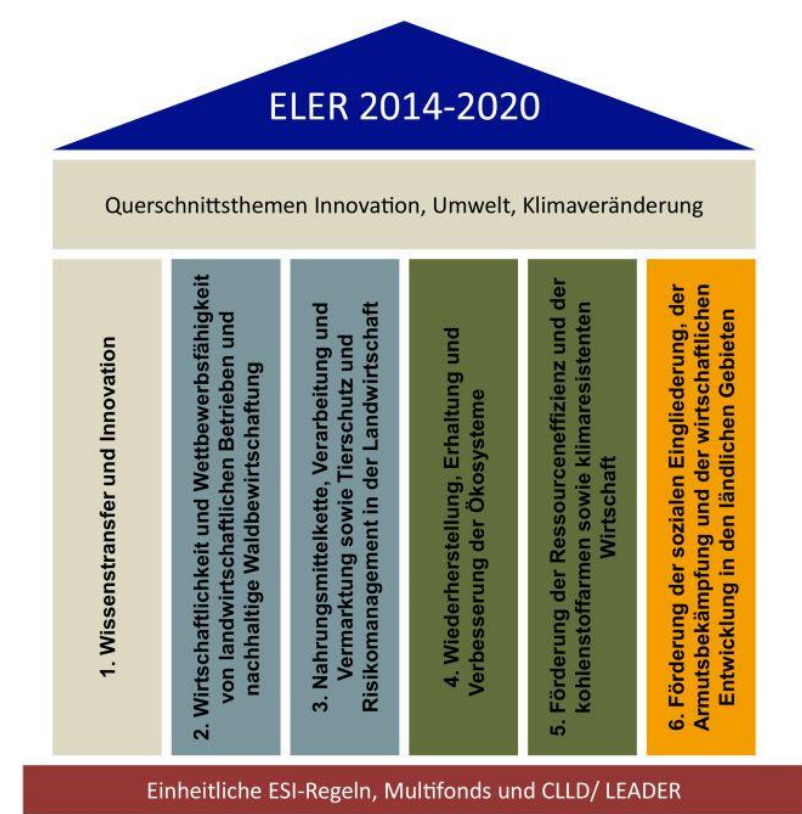

8,3 Mrd. $€+1,1$ Mrd. $€$ nationale Umschichtung + nationale Mittel

cc:dvs*

Abbildung 11: Grafische Darstellung der ELER-Komponenten 2014 - 2020 inkl. finanzielle Ausstattung, Quelle: Deutsche Vernetzungsstelle Ländliche Räume

Um die in Abbildung 11 aufgelisteten Unterziele zu erreichen, sind die Mitgliedsstaaten auf Basis der Verordnung (EU) 1305/2013 gehalten, nationale Programme zur Förderung des ländlichen Raumes aufzustellen. Die vorgenannte Verordnung (EU) 1305/2013 ist Grundlage sämtlicher ELER-Programme, die unter Punkt C die Maßnahmen für Agrarumwelt-/Klimazahlungen, ökologischen Landbau und Natura 2000 beinhalten.

In der Landwirtschaft stellen Agrarumweltmaßnahmen das wichtigste Instrument zur Förderung umweltgerechter Produktionsverfahren mittels finanzieller Anreize dar (Linck et al. in Niens und Marggraf 2010). Gleichzeitig bilden sie das verbindende Element zwischen der Sicherung von Mindeststandards im Rahmen der guten fachlichen Praxis und der Sicherung von ökologisch wertvollen Bereichen in Schutzgebieten (Freese et al. 2011). Der Vertragsnaturschutz in der 
Landwirtschaft kann somit als „Best-practice-Beispiel ${ }^{13 “}$ bezeichnet werden. In der angloamerikanischen Betriebswirtschaftslehre werden so bewährte oder optimale Vorgehensweisen zur Zielerreichung in Unternehmen bezeichnet (Abelshauser 2003).

Es ist davon auszugehen, dass bestimmte Faktoren maßgeblich zum Erfolg dieses Instruments beitragen, die im Wald bislang nicht in dieser Form vorhanden sind. Insofern sollen durch die Analyse der vorliegenden Literatur erfolgsrelevante Gestaltungsmerkmale identifiziert werden. Nachfolgend soll festgestellt werden, welche Umsetzungsmöglichkeiten sich daraus für den Vertragsnaturschutz im Wald ergeben.

\subsubsection{Erfolgsfaktoren Vertragsnaturschutz Landwirtschaft}

Die Agrarumweltmaßnahmen der Gemeinsamen Agrarpolitik der Europäischen Union zur Erhaltung oder Verbesserung des Natur- und Umweltschutzes verpflichten Landwirte bestimmte betriebliche Handlungen durchzuführen, zu dulden oder zu unterlassen. Sie erfreuen sich großer Beliebtheit und sind bspw. in Niedersachsen, gemessen an der Nachfrage, die die programmierten Finanzmittel übersteigt, ein erfolgreiches Instrument des staatlich geförderten Naturschutzes in der Landwirtschaft (s. Fährmann et al. 2018, S. 172). Gemeinhin werden diese Agrarumweltmaßnahmen aufgrund der freiwilligen Teilnahmemöglichkeit für Landwirte als Vertragsnaturschutzmaßnahmen begriffen.

Aus Sicht der an den Agrarumweltmaßnahmen teilnehmenden Landwirte sind mehrere Faktoren für die Attraktivität maßgeblich. Fünf Hauptargumente lassen sich in der wissenschaftlichen Literatur identifizieren.

\section{Die Prämienzahlungen sind in ihrer Höhe angemessen}

Die Höhe der Prämien für Agrarumweltmaßnahmen berechnen sich auf Grundlage der EUHöchstwerte (s. Tabelle 3) nach festgelegten Schemata der teilnehmenden Bundesländer.

Tabelle 3: Übersicht der Förderhöchstsätze aus Anhang II der Verordnung (EU) 1305/2013, zusammengefasst und abgeändert

\begin{tabular}{lc}
\hline Rechtsgrundlage Bezeichnung & Prämienhöchstsatz \\
\hline & $600 €$ je Hektar und Jahr für einjährige \\
& Kulturen \\
& $900 €$ je Hektar und Jahr für mehrjährige \\
Artikel 28 Abs. 8 Agrarumwelt- und Klimamaßnahmen & Kulturen \\
& $450 €$ je Hektar und Jahr für sonstige \\
& Flächennutzung \\
& $200 €$ je Großvieheinheit (GVE) und Jahr für \\
& lokale Tierrassen, die für die Nutzung \\
& verloren gehen könnten \\
\hline
\end{tabular}

\footnotetext{
${ }^{13}$ Im deutschsprachigen Raum auch als Erfolgsmethode bezeichnet
} 
So werden bspw. in Niedersachsen die Fördersätze unter Berücksichtigung der europäischen Höchstsätze und auf Grundlage von Kalkulationen der Landwirtschaftskammer Niedersachsen anhand eines etablierten Deckungsbeitragsschemas für Hoch- und Niederertragsstandorte durchgeführt und aus dem Durchschnitt der Prämiensatz ermittelt. Wenn, wie in der aktuellen Förderperiode, die Deckungsbeiträge aus einer Markthochphase für Getreide ermittelt wurden, kann eine gesteigerte Attraktivität zu Zeiten schwacher Preise beobachtet werden (Fährmann et al. 2018, S. 168). Hinzu kommt, dass für Betriebe auf ertragsschwächeren Standorten oder für Grenzertragsstandorte die Maßnahmen grundsätzlich ökonomisch attraktiv sind, da der durchschnittliche Prämiensatz über dem erzielbaren Ergebnis regulärer Bewirtschaftung liegt (Blomberg 2015).

\section{Der zusätzliche Verwaltungsaufwand ist vertretbar}

Landwirtschaftliche Betriebe in Deutschland sind aufgrund einer Vielzahl von Rechtsvorschriften zur Dokumentation der durchgeführten Maßnahmen, eingesetzten Dünge- und Pflanzenschutzmittel sowie der damit verbundenen Nährstoffbilanzen verpflichtet (vgl. gute fachliche Praxis festgelegt in Düngeverordnung, Pflanzenschutzgesetz, Bodenschutzgesetz).

Auf europäischer Ebene sind erstmals mit der EU VO 1782/2003 (Rat der Europäischen Union 2003) Grundanforderungen in der Gemeinsamen Agrarpolitik definiert worden, die (1) dem Schutz von Gesundheit von Menschen, Tier und Pflanzen, (2) der Umwelt und (3) dem Tierschutz dienen. Diese als Cross-Compliance bezeichneten Regelungen teilen sich auf in sieben Standards zur Erhaltung von Flächen in gutem landwirtschaftlichen und ökologischen Zustand (GLÖZ), bestimmte Regelungen zum Erhalt von Dauergrünland sowie weitere 13 Regelungen zur Grundanforderung an die Betriebsführung (GAB) (Landwirtschaftskammer Niedersachsen 2019). Auch die nationalen Gesetze zählen zu den Cross-Compliance Regelungen. Auf Grundlage der EU VO 1306/2013 ${ }^{14}$ sind die Mitgliedsstaaten gehalten, die bestehenden Regelungen um nationale Standards zu ergänzen. In Deutschland erfolgt dies durch das Agrarzahlungen-Verpflichtungengesetz (AgrarZahIVerpflG) und die Agrarzahlungen-Verpflichtungenverordnung (AgrarZahIVerpflV) (Bundesministerium für Ernährung und Landwirtschaft 2014b).

Zum Erhalt der Direktzahlungen aus der 1. Säule der Europäischen Agrarpolitik bedarf es der Einhaltung dieser Cross-Compliance Regelungen. Insofern ist der aus der Teilnahme an Agrarumweltmaßnahmen resultierende zusätzliche Verwaltungsaufwand aus Sicht der Agrarbetriebe überschaubar (Blomberg 2015). Gegenüber der vergangenen Förderperiode wurde das

\footnotetext{
${ }^{14}$ Europäische Kommission; Europäisches Parlament 17.12.2013.
} 
Baukastensystem der Kombinationsmöglichkeiten von Maßnahmen ausgebaut. Dies sorgt ebenfalls für eine verbesserte Nutzerfreundlichkeit (Fährmann et al. 2018, S. 160).

\section{Die durchgeführten Maßnahmen sind handwerklich nah an der regulären Landwirtschaft}

Die im Ackerbau umsetzbaren Agrarumweltmaßnahmen bestehen maßgeblich aus einer Veränderung der Bodennutzung. Statt des Anbaus von Getreide, Mais oder Ölfrüchten werden streifen- oder flächenweise Blühmischungen eingesät. Die dazu notwendigen Arbeiten entsprechen in der Bodenvorbereitung und Durchführung einer regulären Bestellung. Die Technik ist in den Betrieben vorhanden, die von der normalen Bodenbearbeitung abweichenden zeitlichen Vorgaben ermöglichen sogar eine bessere Ausnutzung der vorhandenen Kapazitäten. Allerdings entsprechen festgelegte Aussaat, Mäh- oder Umbruchtermine der Programme oftmals nicht der sinnhaften Umsetzbarkeit (Niens und Marggraf 2010, S. 32).

\section{Der ökologische Nutzen wird von den Landwirten wahrgenommen}

Die von Landwirten umgesetzten Maßnahmen führen zu einer wahrnehmbaren Förderung der Insekten sowie des Niederwildes. Insbesondere die mehrjährigen Blühstreifen bieten offenlandbewohnenden Tierarten auch im sonst deckungsarmen Herbst und Winter Rückzugsmöglichkeiten zum Schutz vor Wetter und Fressfeinden. Teilweise bestehen Zielkonflikte, wenn innerhalb eines Förderprogramms sowohl Bodenbrüter, wie z. B. das Rebhuhn und gleichzeitig Beutegreifer, wie der Rotmilan gefördert werden.

\section{Generationendenken fördert das Bewusstsein für Belange des Natur- und Umweltschutzes}

Die befragten Landwirte begreifen sich neben ihrer Tätigkeit als Unternehmer auch als Gestalter der Landschaft und haben vor dem Hintergrund des Generationendenkens überwiegend ein hohes Bewusstsein für die Erhaltung der Bodenfruchtbarkeit und ökologisch wertvoller Bereiche (Blomberg 2015).

Wird der Vertragsnaturschutz in der Landwirtschaft auf Grundlage der identifizierten Faktoren als Erfolgsmodell begriffen, gibt es gute Gründe zur Annahme, dass diese Punkte beim Vertragsnaturschutz im Wald bislang nicht in dem notwendigen Maße Berücksichtigung fanden oder umgesetzt wurden. Die weiterführende Analyse der Erfolgsfaktoren von Vertragsnaturschutz im Wald von Erwerbsforstbetrieben ist daher Gegenstand des folgenden Kapitels. Da die befragten Betriebe zum Teil auch Vertragsnaturschutz in der Landwirtschaft umgesetzt haben, wird auch auf diese Maßnahmen ein besonderes Augenmerk gelegt. 


\section{Befragung von privaten Forstbetrieben zum Vertragsnatur- schutz im Wald}

\subsection{Erhebung Potentiale \& Hemmnisse}

In Kapitel 2.3 wurden die gemeinsamen Arbeiten des WaVerNa-Verbundforschungsvorhabens und die ausschließlichen Ergebnisse des Autors zur Erhebung des Status quo von Naturschutzmaßnahmen gegen Entgelt im Wald beschrieben. Die dabei gewonnenen Erkenntnisse stützen sich auf eine offene Onlinebefragung mit 116 auswertbaren Antwortdatensätzen sowie die weiterführende Analyse von 22 bundesweiten Fallbeispielsbetrieben. Es wurden private und kommunale Forstbetriebe befragt.

Zur Erweiterung der Datengrundlage und um die Hypothese zu überprüfen, ob gemischte Betriebe der Land- und Forstwirtschaft aus der Umsetzung von Agrarumweltmaßnahmen über ein gesteigertes Wissen und Kenntnisse für eine erfolgreiche Etablierung eines solchen Instruments in der Forstwirtschaft verfügen, erfolgte eine spezielle Befragung von privaten Erwerbsforstbetrieben, die zum Teil auch erwerbswirtschaftlich in der Landwirtschaft tätig sind. Die Möglichkeit dabei auf eine Gruppe von Betrieben zuzugreifen, die teilweise seit mehreren Jahrzehnten intensiv betrieblich durch die BB Göttingen $\mathrm{GmbH}$ und die Abteilung für Forstökonomie begleitet werden, eröffnete einen leichten Zugang, eine vertrauensvolle Gesprächsgrundlage und die Möglichkeit, neben dem reinen Interviewfragebogen auch bereits vorhandene betriebswirtschaftliche Daten auszuwerten.

\subsubsection{Ziel}

Ziel der Befragung war es, wie bereits oben beschrieben, zu überprüfen, ob gemischte Betriebe der Land- und Forstwirtschaft aus der Umsetzung von Agrarumweltmaßnahmen andere Anforderungen an das Instrument des Vertragsnaturschutzes im Wald stellen als das reine Forstbetriebe tun und ob die Gruppe der „gemischten Betriebe“ über ein gesteigertes Wissen und besondere Kenntnisse für eine erfolgreiche Etablierung eines solchen Instruments in der Forstwirtschaft verfügen. Dazu war es erforderlich, zuerst die Zielsysteme der Betriebsinhaber zu analysieren, ihre Erfahrungen mit Naturschutzmaßnahmen gegen Entgelt in der Landwirtschaft und im Wald zu erfassen und die aus ihrer Sicht für neu aufzulegende Maßnahmen erfolgssteigernden Gestaltungselemente zu identifizieren. Weiterhin sollten die in den Fallbeispielsanalysen des WaVerNa-Projekts identifizierten Erfolgsfaktoren überprüft und unter Bezug auf die unterschiedlichen Betriebsgruppen validiert werden. 


\subsection{Methodik}

\subsubsection{Auswahl der Betriebe}

Die Betriebe, die zur Befragung ausgewählt wurden, sind Mandanten der BB Göttingen GmbH und Teilnehmer an dem dort jährlich durchgeführten Forstbetriebsvergleich. Die Gruppe umfasst 78 Betriebe mit insgesamt rund 105.000 ha, die bundesweit verteilt sind. Diese Betriebe können aufgrund der langjährigen, wiederkehrenden betriebswirtschaftlichen Auswertungen und Kennzahlenanalysen als Panel (s. Kromrey 2009, S. 366) betriebswirtschaftlich besonders interessierter Forstbetriebe verstanden werden. Somit können die Methoden der Paneldatenbefragung angewendet werden. Die von der BB Göttingen GmbH erhobenen Daten sind zweidimensional, da sie sowohl zeitlich (vertikal) als auch betriebsübergreifend (horizontal) kontinuierlich erhoben werden. ${ }^{15}$ Es liegen demnach einzelbetrieblich eine große Vielzahl von betrieblichen Daten vor, die, unter Berücksichtigung des Datenschutzes und der absoluten Vertraulichkeit, dem Autor zugänglich sind.

\subsubsection{Befragung der BB-Betriebe}

Die Befragung der Betriebe zum Thema „Naturschutz gegen Entgelt im Wald \& in der Landwirtschaft" wurde mittels eines per E-Mail versandten, strukturierten Fragebogens vorbereitet. Die Empfänger wurden über den Emailverteiler des BB-Forstbetriebsvergleichs erreicht. Nach Erhalt des Fragebogens erfolgte eine telefonische Kontaktaufnahme mit den Empfängern, um das leitfadengestützte Experteninterview durchzuführen. Die direkte Form der Kommunikation in Form eines strukturierten Interviews ermöglicht es dem Befragten, Unklarheiten in der Fragestellung direkt mit dem Befrager zu klären und somit die Qualität der Antworten zu verbessern (Atteslander 2010). Gleichzeitig kann der Befrager bei einzelnen Fragen nachhaken und aus den getätigten Antworten heraus weitere Erkenntnisse erfassen, die in einer rein schriftlichen Befragung nicht möglich gewesen wären. Die Vorabübersendung des Fragebogens gibt dem Befragten die Möglichkeit komplexe Fragen vorzubereiten und das zur Beantwortung erforderliche Material herauszusuchen. Da das Ziel der Befragung auf die Erfassung von fachlichen Inhalten und nicht die Erfassung von spontanen Reaktionen ausgelegt war, besteht nur ein geringes Risiko der Antwortverzerrung durch die Vorabübersendung (Mayring 2008). Ein generelles methodisches Problem des Experteninterviews rührt aus dem direkten Kontakt der Gesprächspartner.

${ }^{15}$ Da die BB Göttingen GmbH als Unternehmensberatung in diesen Betrieben Beratung zur Aufwandsund Ertragsoptimierung durchführt, handelt es sich, genau genommen, nicht um Beobachtungsdaten in der klassischen Begriffsverwendung der Paneldatenanalyse, da eine direkte Intervention durch den Beobachter im Zuge von betriebswirtschaftlicher Beratung stattfindet. 
Mieg (2005, S. 5) beschreibt es wie folgt: „Es ist unsicher, ob nicht Befragte in einem Interview mit einem anderen Interviewer eine andere Geschichte berichten würden."

Aufgrund der beruflichen Tätigkeit des Autors dieser Arbeit als betriebswirtschaftlicher Berater einiger der befragten Betriebe ist von einer Beeinflussung der Antworten auszugehen (s. Krott und Suda 2001, S. 127), die für die Qualität der Antworten als sehr vorteilhaft angesehen wird, da ein langjähriges Vertrauensverhältnis besteht. Die Experten können also frei und ungezwungen antworten, ohne sich über die Intention des Befragers Gedanken machen zu müssen. Der Verzerrungseffekt der sozialen Erwünschtheit wurde unter Berücksichtigung des Befragungsziels Vertragsnaturschutz als gering eingestuft.

Das bestehende Vertrauensverhältnis spiegelt sich auch in der hohen Rücklaufquote von 64 \% der auswertbaren Datensätze wider. ${ }^{16}$ Von 78 angeschriebenen Betrieben konnten 50 Betriebe zu einem Interview gewonnen und letztlich 40 Betriebe ausgewertet werden. Die mangelnde Auswertbarkeit der anderen Betriebe lag zumeist in der Nichtdurchführung von Vertragsnaturschutzmaßnahmen begründet.

\subsubsection{Aufbau des Fragebogens}

Der für diese Arbeit entwickelte Fragebogen war Grundlage für die mündlichen Befragungen. Diese erfolgten in Form von Experteninterviews, da die Betriebsinhaber und -leiter aufgrund ihrer Funktion und beruflichen Erfahrung als Experten angesehen werden können. Der vorab übersendete Fragebogen dient neben der Vorbereitung beider Seiten auch als strukturgebender Leitfaden für das Gespräch. Er bestand aus vier Themenblöcken.

Im ersten Schritt wurden allgemeine Daten über die Funktion des Interviewten und die Ziele des Betriebs in der Land- und Forstwirtschaft erhoben. Allgemeine Betriebsdaten konnten aus den vorliegenden Informationen des Betriebsvergleichspanels entnommen werden. Allgemeine Fragen zum Interviewten erfolgten dabei in offener Form, die Fragen zu den Zielen der Betriebe wurden überwiegend als geschlossene Fragen formuliert, wobei ergänzende Antworten möglich waren. Auf Basis vorgegebener möglicher Betriebsziele konnten die Befragten auf einer sog. Likert-Skala ${ }^{17}$ (Krüger 2013) jeweils die passenden Werte auswählen. Die gewählte Skala wies sechs Werte auf, um eine regelmäßige neutrale Auswahl des Antwortenden zu vermeiden ${ }^{18}$. Die

\footnotetext{
${ }^{16}$ Auch dieser Verzerrungseffekt wird bei Krott und Suda 2001, S. 120 beschrieben, in der vorliegenden Arbeit jedoch als klare Vorteilhaftigkeit bewertet.

17 Benannt nach dem Sozialforscher Rensis Likert

${ }^{18}$ Die Frage nach einer geraden oder ungeraden Skalierung ist Gegenstand vieler Publikationen und Lehrbücher im Bereich der empirischen Sozialforschung (vgl. Atteslander 2010, Kopp 2012) und wird nicht abschließend beantwortet. Je nach Ziel der Befragung und antizipierter Antworten kann jeweils die eine
} 
Betriebsziele konnten unabhängig voneinander gewichtet werden, so dass auch eine Mehrfachvergabe bspw. der höchsten oder niedrigsten Werte möglich war. Zusätzlich bestand für die Befragten die Möglichkeit weitere, nicht in der Befragung vorgesehene Betriebsziele anzugeben. Hiervon wurde jedoch kein Gebrauch gemacht.

Der zweite Komplex des Fragebogens hatte vergangene Naturschutzmaßnahmen gegen Entgelt im Wald und in der Landwirtschaft und die zugrundeliegenden speziellen Zielsetzungen des Befragten zum Thema. Weiterhin wurden aufgetretene Probleme aber auch potenzielle Stärken erfasst. Dabei wurden die Fragen, die sich auf durchgeführte Maßnahmen bezogen, als offene Fragen formuliert. Dies resultierte aus der Erfahrung der Onlinebefragung \& der Fallbeispielsanalysen des WaVerNa-Projekts, in dem deutlich wurde, dass bundesweit aufgrund der föderalen Zuständigkeiten eine Vielzahl von Maßnahmen (teilweise auch nur mit unterschiedlichen Bezeichnungen bei gleichen Inhalten) mit unterschiedlichen Partnern im Rahmen verschiedener Förder-/Vertragsprogramme durchgeführt wurden.

Der dritte Themenbereich wendete sich zukünftigen Maßnahmen zu. Die Befragten sollten sich zu Verbesserungsmöglichkeiten verschiedener Aspekte von Vertragsnaturschutzmaßnahmen äußern. Befragungsteilnehmer, die neben dem Forstbetrieb auch einen landwirtschaftlichen Betrieb bewirtschaften, wurden weiterhin zu Stärken \& Problemen von Agrarumweltmaßnahmen befragt. Die Fragebogen schloss mit der Frage, ob den Teilnehmern besonders positive Beispiele bekannt wären, aus deren Erfahrungen Ableitungen getroffen werden könnten.

Diese Fragen wurden bewusst offen formuliert, um den Befragten die Möglichkeit zu geben, möglichst viele eigene Gedanken und Sichtweisen anzusprechen.

\subsubsection{Durchführung der Befragung}

Zwei Wochen nach Übersendung des Fragebogens per Emailverteiler wurden die Befragten telefonisch kontaktiert. Während der Befrager (der Autor dieser Arbeit) sich maßgeblich auf das Stellen der Fragen beschränkte, wurde den Befragten viel Raum gelassen, um möglichst umfangreich Informationen zu den Themenbereichen geben zu können. Der Befrager dokumentierte dabei auf Grundlage des Fragebogens die Inhalte der einzelnen Gespräche. Insgesamt konnten von den 78 kontaktierten Betrieben 50 Betriebe zu einem Interview gewonnen werden, wobei letztlich 40 vollständige Datensätze in die Auswertung in dieser Arbeit eingeflossen sind.

oder die andere Variante vorteilhaft sein. In dieser Befragung sollte die „gleichgültige“ Haltung vermieden werden, um eine klare Positionierung der Befragten zu erzwingen. 


\subsection{Ergebnisse}

\subsubsection{BB-Forstbetriebe}

Die 40 ausgewerteten Betriebe verteilen sich in ihrer räumlichen Struktur über die Bundesländer Schleswig-Holstein, Mecklenburg-Vorpommern, Brandenburg, Niedersachsen, Sachsen-Anhalt, Thüringen, Sachsen, Hessen, Nordrhein-Westfalen \& Bayern. Damit sind 10 von 13 relevanten Flächenländern exemplarisch vertreten ${ }^{19}$, nicht erfasst werden Betriebe aus den Bundesländern Rheinland-Pfalz, Saarland und Baden-Württemberg.

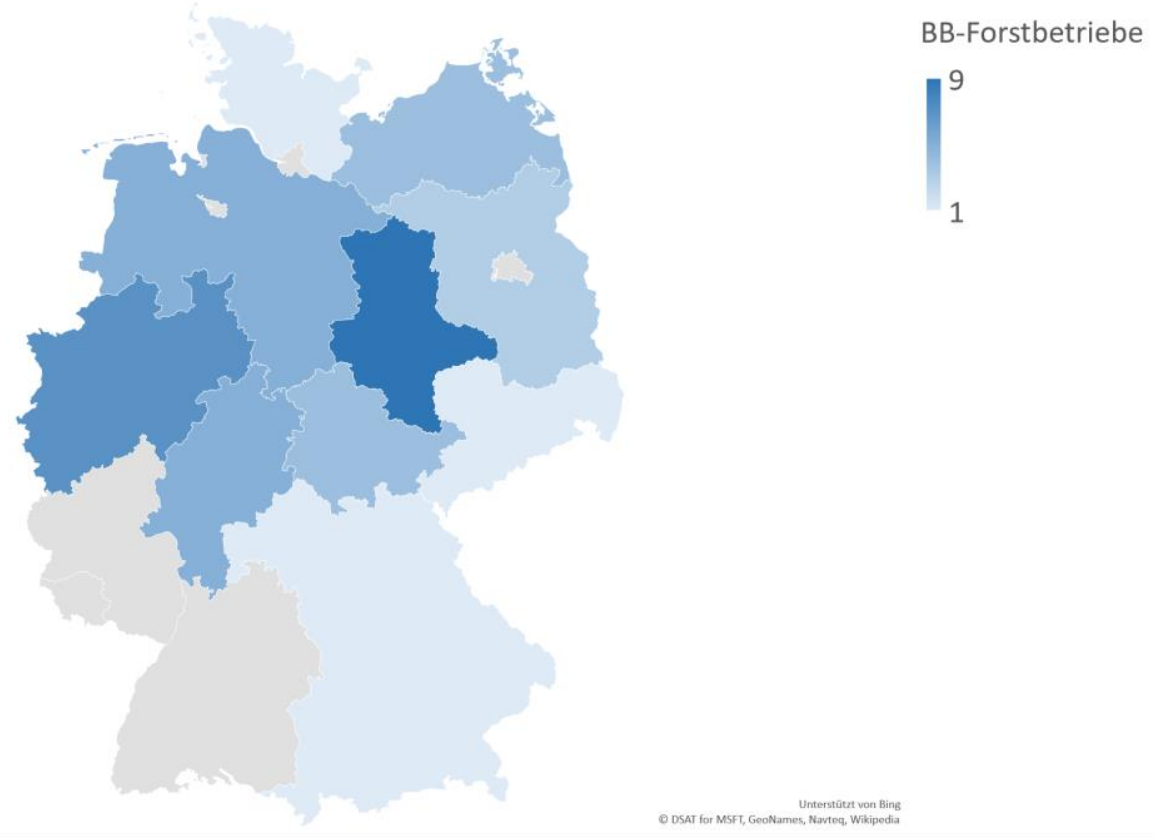

Abbildung 12: Lage der befragten BB-Forstbetriebe nach Bundesländern, $n=40$

Von den 40 befragten Betrieben wurde in 19 Fällen der Eigentümer, der zeitgleich auch betriebsführend tätig war, befragt. Im Durchschnitt bewirtschaftet dieser 760 ha Waldfläche. Die 8 befragten Betriebsleiter repräsentieren durchschnittlich 3.831 ha Fläche. In 12 Fällen wurden externe forstliche Dienstleister in Wahrnehmung der Eigentümeraufgaben befragt. Diese Betriebe sind durchschnittlich 688 ha groß.

\footnotetext{
${ }^{19}$ Die Stadtstaaten Bremen, Hamburg und Berlin sind aufgrund der geringen Waldflächen, die sich zudem zumeist im Stadteigentum befinden, von keiner großen Relevanz für Naturschutzmaßnahmen gegen Entgelt im Wald. Das Land Bremen bspw. hat in der Vergangenheit stets das Regionalförderprogramm Niedersachsens mit übernommen (s. Niedersächsisches Ministerium für Ernährung, Landwirtschaft und Verbraucherschutz 2014)
} 


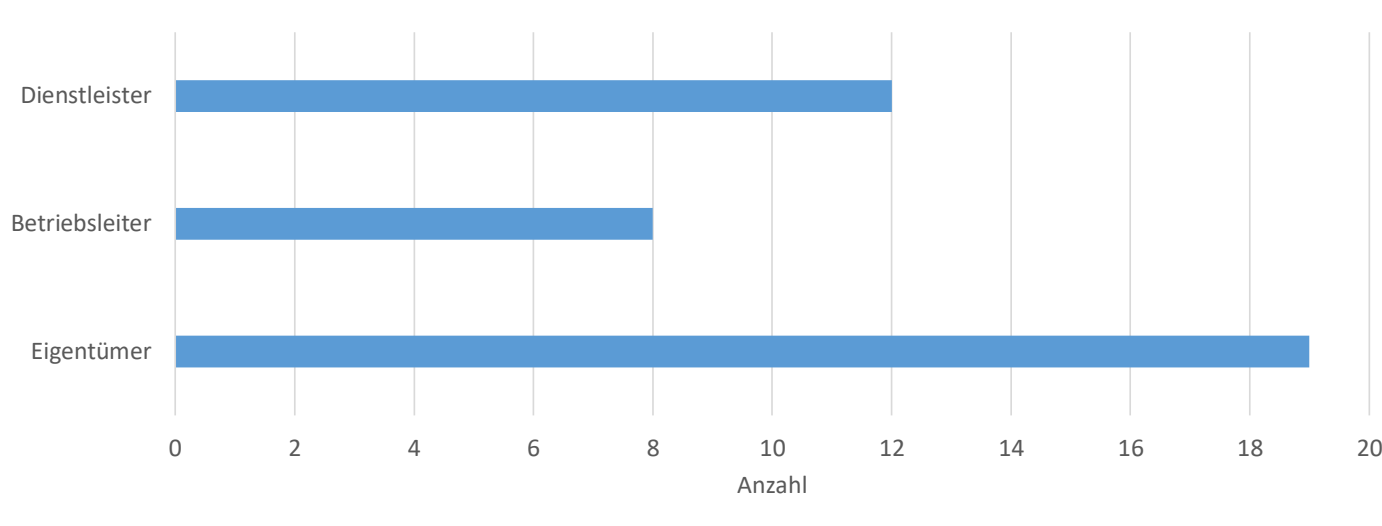

- Funktion des Antwortenden

Abbildung 13: Funktion der Befragten $(n=38)$

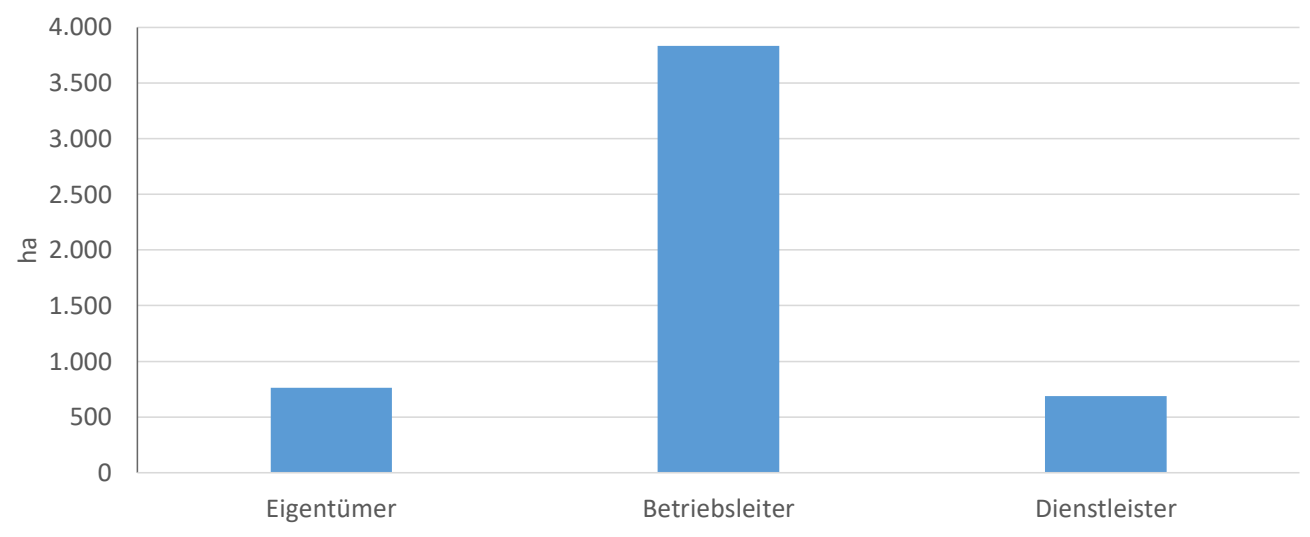

Abbildung 14: Durchschnittliche Betriebsfläche nach Funktion ( $n=40)$

Die Größenspanne der Forstbetriebe liegt zwischen 127 ha bis 12.500 ha. Insgesamt liegen jedoch nur vier Betriebe über einer Größe von 2.000 ha (s. Abbildung 15). Der Mittelwert aller Forstbetriebe liegt bei 1.409 ha, der Median bei 828 ha. Im Vergleich zu allen BB-Forstbetrieben mit einer durchschnittlichen Größe von 1.412 ha entspricht die ausgewertete Gruppe genau der Gesamtheit (Blomberg et al. 2019).
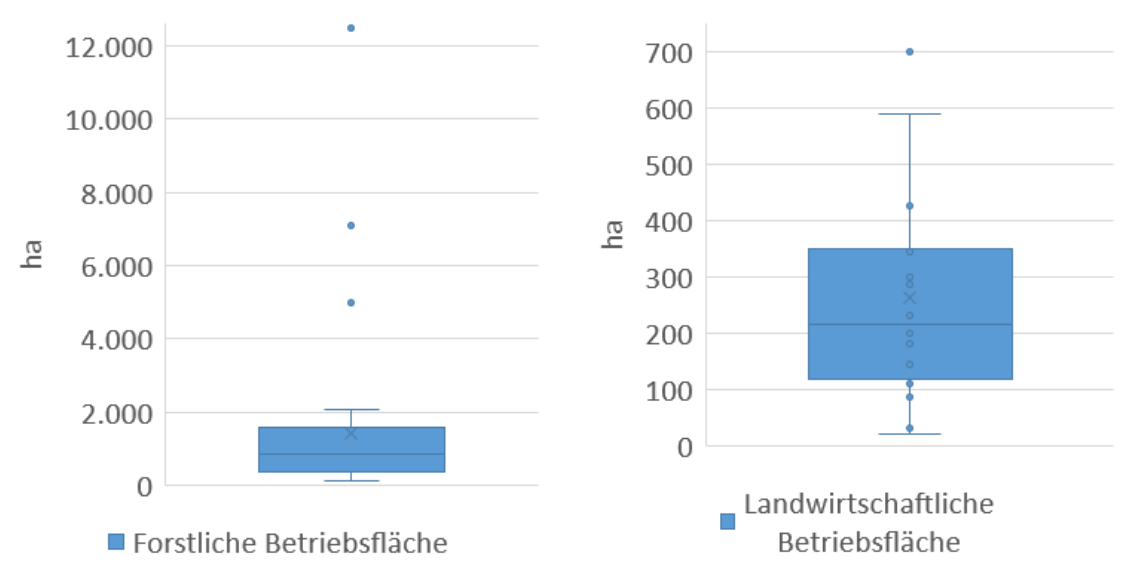

Abbildung 15: Größenverteilung Forstbetriebsfläche alle Betriebe $(n=40)$ \& landwirtschaftliche Fläche nur Gemischtbetriebe $(n=16)$. 
Die landwirtschaftliche Fläche der gemischten Betriebe liegt wesentlich enger beisammen. Bei einer Spanne von 20 ha bis 700 ha liegt der Mittelwert bei 262 ha, der Median bei 215 ha. Sowohl im Forst als auch in der Landwirtschaft führen einige Ausreißer nach oben zu der Differenz zwischen arithmetischem Mittelwert und Median (s. Abbildung 15).

In Anlehnung an die Auswertungen des BB-Forstbetriebsvergleichs wurden auch die Betriebe der Befragung in die drei Baumartengruppen Laubholz (22 Betriebe), Fichte (11 Betriebe) und Kiefer (7 Betriebe) aufgeteilt. Die Verteilung der befragten Betriebe deckt sich mit der Zusammensetzung im gesamten Betriebsvergleich ${ }^{20}$. In Abbildung 16 sind die durchschnittlichen Baumartenanteile der Gruppen aufgetragen. In der häufigsten Gruppe der Laubholzbetriebe überwiegt definitionsgemäß die Baumart Buche (inkl. der sonstigen Laubholzarten außer Eiche) mit 54 \% Flächenanteil, dennoch verfügen diese Betriebe mit 26 \% Fichte (inkl. Tanne \& Douglasie) auch noch über rund ein Viertel ihrer Fläche produktive Nadelhölzer. ${ }^{21}$

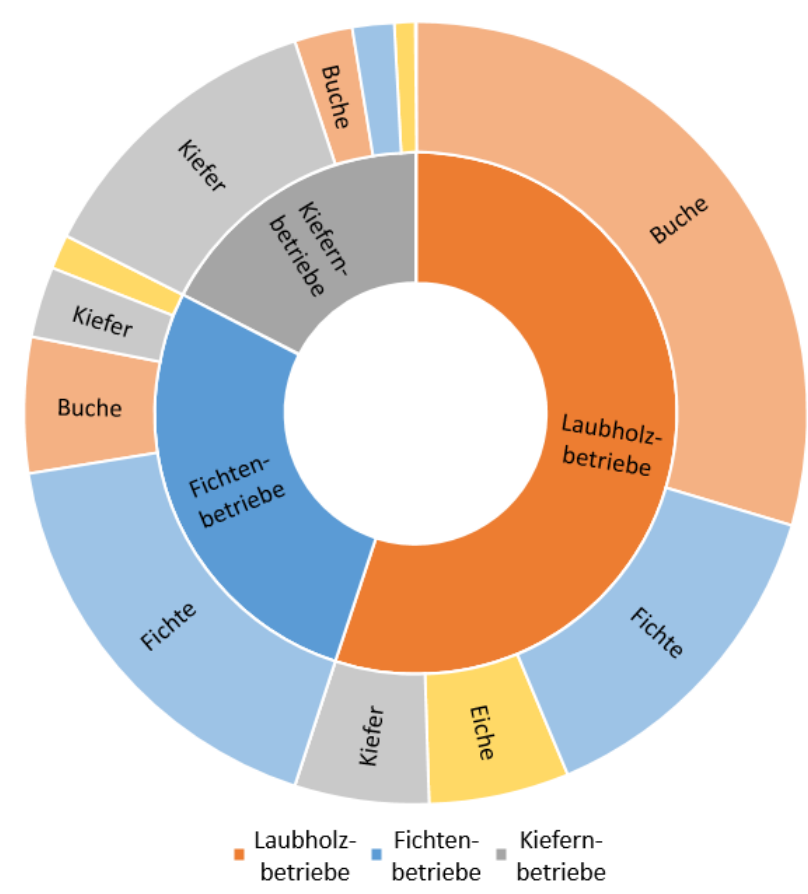

Abbildung 16: Baumartenanteile in den Baumartengruppen Laubholz $(n=22)$, Fichte $(n=11)$ und Kiefer $(n=7)$

\footnotetext{
${ }^{20}$ Weitergehend kann angenommen werden, dass die Laubholzbetriebe häufiger von Natura 2000-Gebieten betroffen sind, da sich die zu schützenden Lebensraumtypflächen in Deutschland vorrangig auf Laubhölzer beziehen (LRT 9110 - Hainsimsenbuchenwald, 9130 - Waldmeisterbuchenwald, 9160 - Sternmieren-Eichen-Hainbuchenwald) (Rosenkranz et al. 2012). Somit besteht auch ein gesteigerter Bedarf nach finanziellem Ausgleich.

${ }^{21}$ Die Befragung wurde im Herbst 2018 durchgeführt als der Sturm Friederike vom Januar 2018 bereits nennenswerte Schäden hinterlassen hatte, die Ausmaße der anschließenden, dürrebedingten Käferkalamitäten bei weitem noch nicht abschätzbar waren. Es ist davon auszugehen, dass größere Teile der Fichtenflächen nach aktuellem Stand (April 2020) zerstört oder unbestockt sind.
} 
In der Fichten- und der Kieferngruppe überwiegt die namensgebende Baumart mit jeweils $64 \%$ bzw. 72 \% Flächenanteil. Somit stellt die Laubholzgruppe, die bezüglich der Baumartenverteilung am stärksten gemischte Gruppe dar.

\subsubsection{Ertragssituation der BB-Forstbetriebe}

In Zusammenarbeit mit der Abteilung Forstökonomie der Georg-August-Universität Göttingen entsteht bei der BB Göttingen $\mathrm{GmbH}$ jährlich der bundesweit größte, privatwirtschaftlich organisierte Forstbetriebsvergleich (vgl. Blomberg et al. 2019). Die in der vorliegenden Arbeit befragten Forstbetriebe sind Teilnehmer dieses Vergleichs und werden zum Teil bereits seit vielen Jahren von der BB Göttingen $\mathrm{GmbH}$ betriebswirtschaftlich betreut. Nachfolgend wird die wirtschaftliche Situation der Betriebe des Jahres 2017 analysiert. Das Jahr 2017 ist das letzte vollständig ausgewertete Wirtschaftsjahr vor der Befragung und es war noch unbetroffen von den Stürmen, Borkenkäferkalamitäten und Marktverwerfungen, die seit Januar 2018 den Forstsektor stark getroffen haben. Innerhalb des Betriebsvergleichs werden die Betriebe anhand des Betriebsergebnisses unterteilt in den Durchschnitt, das erste Quartil (Obere 25\%) mit den höchsten Ergebnissen und das letzte Quartil (Untere 25\%).

Im Durchschnitt (s. Tabelle 4) sind die an der Befragung teilnehmenden Betriebe 1.186 ha groß. Damit liegen sie weit über dem bundesdeutschen Privatwalddurchschnitt von 3 ha (Polley und Hennig 2015, S. 34). Sie sind damit klar der Gruppe der Erwerbsforstbetriebe zuzurechnen, die zumindest einen nennenswerten Anteil des jährlichen Eigentümereinkommens aus der Bewirtschaftung von Wald erzielen. Bei einem durchschnittlichen Hiebsatz von 5,6 Efm/ha wurden im Jahr 2017 6,7 Efm/ha eingeschlagen. Das entspricht einer Nutzung über Hiebsatz von rund 19,6\%, wobei diese moderate Hiebssatz-Überschreitung als nachhaltig eingeschätzt wird, da der Hiebssatz traditionell das nachhaltige Nutzungspotential, insbesondere in den zielstarken Beständen, unterschätzt (vgl. Böckmann 2016, S. 35). Der höchste Einschlagsanteil lag dabei mit $43 \%$ in der Fichte, gefolgt vom Laubholz mit $30 \%$. Nur ein geringer Teil des Einschlags wird dabei durch eigenes Forstpersonal erledigt ( $5 \%$ im Durchschnitt), der Haupteinschlag wird durch Unternehmer im Lohn (66 \%) oder auf dem Stock (28 \%) erledigt. 
Tabelle 4: Naturaldaten befragte BB-Forstbetriebe Wirtschaftsjahr 2017 ( $n=38$, die Abweichung zu der Gesamtzahl von 40 Betrieben resultiert aus zwei Betrieben, die in 2017 nicht am Forstvergleich teilgenommen haben), getrennt nach Baumartengruppen sowie nach Obere 25\%, Durchschnitt aller Betriebe und Untere 25\%, Quelle: BB-Forstbetriebsvergleich 2017

\begin{tabular}{|l|r|r|r|r|r|r|}
\hline & Laubholz & Fichte & Kiefer & $\begin{array}{c}\text { Obere } \\
\mathbf{2 5 \%}\end{array}$ & Mittel & $\begin{array}{c}\text { Untere } \\
\mathbf{2 5 \%}\end{array}$ \\
\hline Holzbodenfläche ha & 1.446 & 897 & 898 & 536 & 1.186 & 704 \\
\hline Holzvorrat fm/ha & 255 & 301 & 231 & 279 & 264 & 210 \\
\hline Hiebsatz fm/ha & 5,6 & 6,6 & 4,2 & 6,2 & 5,6 & 4,9 \\
\hline Einschlag fm/ha & 5,9 & 7,4 & 7,9 & 9,2 & 6,7 & 4,8 \\
\hline davon Stammholz & $23 \%$ & $23 \%$ & $8 \%$ & $24 \%$ & $20 \%$ & $19 \%$ \\
\hline davon Abschnitte & $30 \%$ & $49 \%$ & $52 \%$ & $42 \%$ & $40 \%$ & $30 \%$ \\
\hline davon Industrieholz & $36 \%$ & $24 \%$ & $37 \%$ & $30 \%$ & $33 \%$ & $41 \%$ \\
\hline davon Energieholz & $11 \%$ & $4 \%$ & $3 \%$ & $4 \%$ & $7 \%$ & $10 \%$ \\
\hline davon Buche & $44 \%$ & $19 \%$ & $5 \%$ & $25 \%$ & $30 \%$ & $44 \%$ \\
\hline davon Fichte & $37 \%$ & $73 \%$ & $11 \%$ & $51 \%$ & $43 \%$ & $28 \%$ \\
\hline davon Kiefer & $13 \%$ & $6 \%$ & $82 \%$ & $23 \%$ & $24 \%$ & $20 \%$ \\
\hline Eigenregie & $9 \%$ & $2 \%$ & & $8 \%$ & $5 \%$ & $6 \%$ \\
\hline Unternehmer & $71 \%$ & $78 \%$ & $33 \%$ & $57 \%$ & $66 \%$ & $70 \%$ \\
\hline Selbstwerber & $19 \%$ & $20 \%$ & $67 \%$ & $35 \%$ & $28 \%$ & $25 \%$ \\
\hline
\end{tabular}

Aus dem Einschlag von 6,7 Efm/ha resultiert ein Holzertrag von im Mittel $370 € /$ ha (s. Tabelle 5). Bezogen auf den Gesamtertrag von $403 €$ /ha entspricht das einem Anteil von $92 \%$ aus der Nutzung von Holz. Fördermittel, unter die in diesem Betriebsvergleich auch Zahlungen der öffentlichen Hand für Vertragsnaturschutzmaßnahmen fallen, machen lediglich $4 € /$ ha und damit weniger als $1 \%$ des Betriebsertrags aus.

Tabelle 5: Ertrags- und Aufwandsstruktur befragte BB-Forstbetriebe Wirtschaftsjahr 2017 in $€ / h a$ ( $n=38$ ), getrennt nach Baumartengruppen sowie nach Obere 25\%, Durchschnitt aller Betriebe und Untere 25\%, Quelle: BB-Forstbetriebsvergleich 2017

\begin{tabular}{|c|c|c|c|c|c|c|}
\hline$€ /$ ha & Laubholz & Fichte & Kiefer & $\begin{array}{c}\text { Obere } \\
25 \%\end{array}$ & Mittel & $\begin{array}{c}\text { Untere } \\
25 \%\end{array}$ \\
\hline Holzerlöse & 337 & 467 & 311 & 503 & 370 & 247 \\
\hline Lagerbestand Anfang & 8 & 12 & 0 & 6 & 8 & 14 \\
\hline Lagerbestand Ende & 7 & 15 & & 12 & 8 & 12 \\
\hline Jagd & 17 & 13 & 12 & 11 & 15 & 18 \\
\hline Leistungen $\mathrm{f}$. Dritte & 7 & 1 & & 2 & 4 & 5 \\
\hline Förderung & 8 & 1. & 0 & 1 & 4 & 12 \\
\hline Sonstiges & 13 & 6 & 1 & 3 & 9 & 20 \\
\hline Ertrag & 381 & 491 & 325 & 526 & 403 & 300 \\
\hline Materialkosten & 8 & 6 & 5 & 6 & 7 & 9 \\
\hline Lohnkosten/Lohnansatz & 23 & 19 & 3 & 15 & 18 & 20 \\
\hline Maschinenkosten & 4 & 3 & 4 & 5 & 4 & 4 \\
\hline Unternehmeraufwand & 111 & 142 & 70 & 127 & 113 & 89 \\
\hline Gehälter, Betreuung, Verwaltung & 51 & 53 & 32 & 50 & 48 & 45 \\
\hline So. Allgemeinkosten & 65 & 50 & 35 & 45 & 55 & 74 \\
\hline Summe Aufwand & 262 & 274 & 149 & 248 & 245 & 240 \\
\hline Betriebsergebnis & 119 & 217 & 176 & 278 & 158 & 59 \\
\hline Betriebsergebnis hiebsatzbereinigt & 102 & 177 & 48 & 156 & 114 & 58 \\
\hline
\end{tabular}


Den jährlich höchsten Anteil der Aufwendungen nehmen die Unternehmerkosten für Holzeinschlag, Pflanzung und Pflege ein. Die Allgemein-/Fixkosten (Gehälter, Betreuung, Verwaltung \& sonstige Allgemeinkosten) machen mit $103 € /$ ha rund 42 \% des Gesamtaufwands aus. Die anderen $58 \%$ verteilen sich auf die operativen Kosten.

Bei einem Gesamtertrag von $403 € /$ ha und einem Gesamtaufwand von $245 €$ /ha verbleibt den befragten Betrieben ein Betriebsergebnis von $158 € /$ ha. Unter Annahme, dass die Betriebe auf dem Niveau ihres Hiebsatzes ${ }^{22}$ geschlagen hätten und bis auf die unmittelbar einschlagsbezogenen Erträge und Aufwendungen alle sonstigen unverändert sind, bliebe ein hiebsatzbereinigtes Betriebsergebnis von $114 € /$ ha. Da das Betriebsergebnis in den Forstbetrieben besonders von der Höhe des Einschlags abhängt, lohnt der Blick auf die hiebsatzbereinigten Ergebnisse der Oberen 25\% und der Unteren 25\%. Die Hiebsatzbereinigung zeigt deutlich, dass die Oberen 25\% ihr gutes Ergebnis von $278 €$ /ha einer Nutzung über Hiebsatz zu verdanken haben, wohingegen die Unteren $25 \%$ durch die Hiebsatzbereinigung annähernd auf gleichem Niveau bleiben. Ihr geringes Ergebnis resultiert somit entweder aus geringeren Deckungsbeiträgen in Folge schlechterer Sortierung, ungehobener Potentiale der Aushaltung oder höherer Kosten durch ineffiziente Arbeitsprozesse. Bezogen auf die Baumartengruppen wirkt sich die Hiebsatzbereinigung besonders bei den Kiefernbetrieben sehr stark aus, die im Jahr 2017 deutlich mehr als nach Forsteinrichtung vorgesehen, eingeschlagen haben (7,9 Efm/ha statt 4,2 Efm/ha).

In Tabelle 6 ist die Ermittlung des Deckungsbeitrags I, des erntekostenfreien Holzerlöses, auf Basis des Kostenträgers Erntefestmeter für die befragten Betriebe dargestellt. Über alle Baumarten, Sortimente und Werbungsarten konnte nach Abzug der Holzerntekosten ein DB I von $38 € /$ Efm erzielt werden.

Tabelle 6: DB I (erntekostenfreier Holzerlös) der befragten BB-Forstbetriebe Wirtschaftsjahr 2017 (n=38), getrennt nach Baumartengruppen sowie nach Obere 25\%, Durchschnitt aller Betriebe und Untere 25\%, Quelle: BB-Forstbetriebsvergleich 2017

\begin{tabular}{|l|r|r|r|r|r|r|}
\hline \multicolumn{1}{|c|}{$\boldsymbol{\epsilon} / \mathrm{Efm}$} & Laubholz & Fichte & Kiefer & $\begin{array}{c}\text { Obere } \\
\mathbf{2 5 \%}\end{array}$ & \multicolumn{1}{c|}{ Mittel } & \multicolumn{1}{c|}{$\begin{array}{l}\text { Untere } \\
\mathbf{2 5 \%}\end{array}$} \\
\hline Holzerlös Regie/Untern. & $\mathbf{6 2}$ & $\mathbf{7 4}$ & $\mathbf{4 7}$ & $\mathbf{6 8}$ & $\mathbf{6 2}$ & $\mathbf{5 1}$ \\
\hline Erntekosten Regie/Untern. & 19,13 & 21,94 & 14,34 & 19,57 & 19,06 & 16,44 \\
\hline Erntekostenfreier Holzerlös & $\mathbf{4 2}$ & $\mathbf{5 2}$ & $\mathbf{3 3}$ & $\mathbf{4 8}$ & $\mathbf{4 3}$ & $\mathbf{3 4}$ \\
\hline Erlös Stockverkauf/Selbstw. & 24 & 21 & 27 & 30 & 24 & 21 \\
\hline Deckungsbeitrag insgesamt & $\mathbf{3 9}$ & $\mathbf{4 6}$ & $\mathbf{2 9}$ & $\mathbf{4 2}$ & $\mathbf{3 8}$ & $\mathbf{3 1}$ \\
\hline
\end{tabular}

\footnotetext{
${ }^{22}$ Es ist zu beachten, dass der Hiebsatz in privaten Forstbetrieben häufig durch steuerliche Effekte beeinflusst wird und somit unterhalb der objektiven, nachhaltigen Nutzungsmöglichkeit liegt. Die Eignung als Indikator nachhaltiger Nutzung ist somit nur bedingt gegeben.
} 
Die Fichte hat in der Vergangenheit eine entscheidende Rolle für die erwerbswirtschaftlich orientierten Betriebe gespielt. ${ }^{23}$ Nachfolgend soll dies an den Kennzahlen der befragten Betriebe deutlich gemacht werden. Zunächst ist in Abbildung 17 dargestellt, dass keine Korrelation zwischen dem Fichtenanteil an der Holzbodenfläche und der Höhe des Einschlags vorliegt. Gleichwohl besteht erwartungsgemäß ein positiver Zusammenhang von Fichtenanteil an der Holzbodenfläche und Fichtenanteil am Einschlag. Mittels Korrelationsanalyse lässt sich ein pearson'scher Korrelationskoeffizient von $r=0,87$ mit einem $\mathrm{p}$-Wert kleiner 0,05 ermitteln.
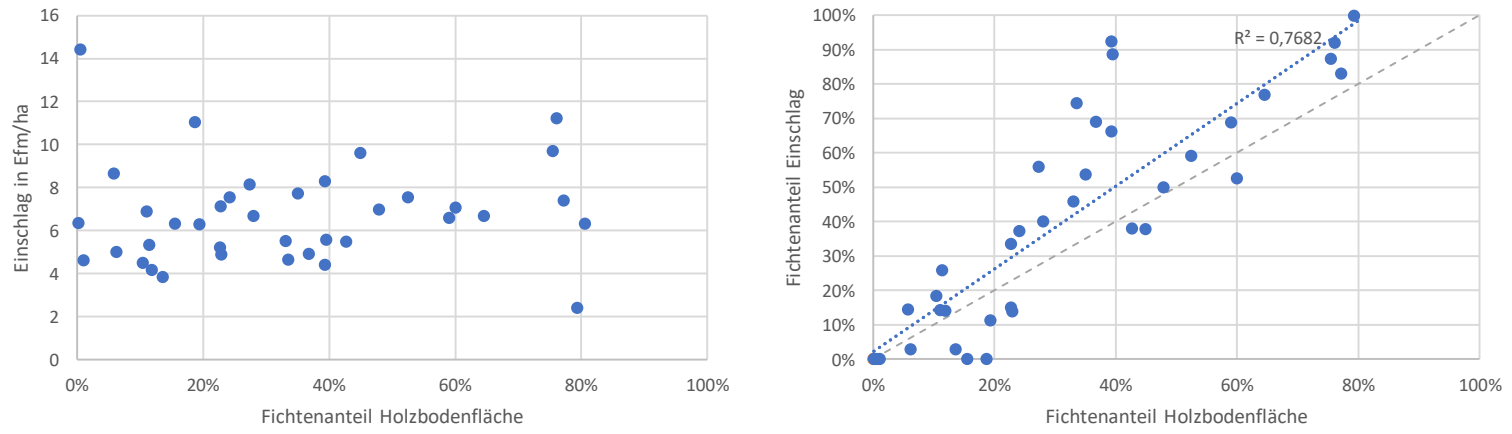

Abbildung 17: Zusammenhang von Fichtenanteil an der Holzbodenfläche und Einschlag sowie Zusammenhang Fichtenanteil an der Holzbodenfläche und Fichtenanteil am Einschlag, $n=38$

Der Einfluss der Fichte auf den wirtschaftlichen Erfolg der Betriebe ist in Abbildung 18 dargestellt. Je höher der Einschlagsanteil der Fichte ausfällt, desto höher ist der erntekostenfreie Holzerlös. Der Korrelationskoeffizient beträgt $r=0,72$ mit $p<0,05$. Für die anderen Baumartengruppen ist ein solcher Zusammenhang auf Basis der vorliegenden Daten nicht nachweisbar, die Korrelationen sind nicht signifikant (s. Abbildung 19).

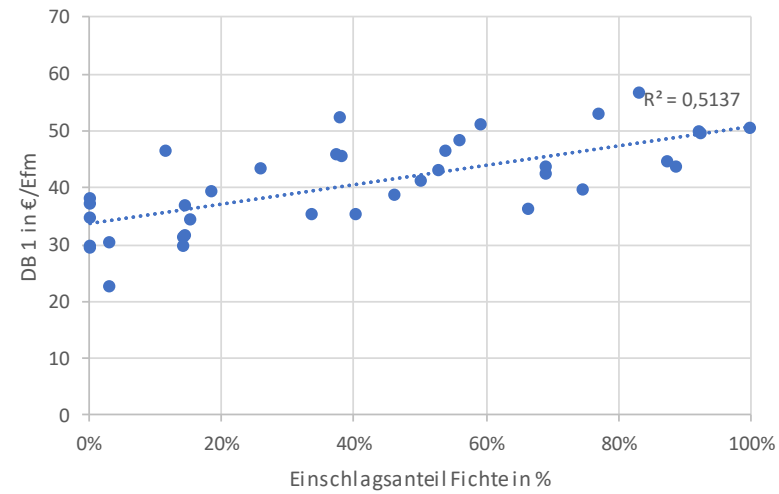

Abbildung 18: Zusammenhang von Einschlagsanteil der Baumartengruppe Fichte und erzieltem Deckungsbeitrag I (erntekostenfreier Holzerlös), $n=38$

${ }^{23}$ Die Daten des Jahres 2017 stehen hier stellvertretend für zumindest die vorangegangenen 10 Jahre. Aktuell haben die starken Marktverwerfungen in Folge der Stürme und Dürren dazu geführt, dass diese Zusammenhänge nicht gelten. Es ist jedoch davon auszugehen, dass eine Beruhigung der Märkte einen ähnlichen Status quo wiederherstellen wird. 

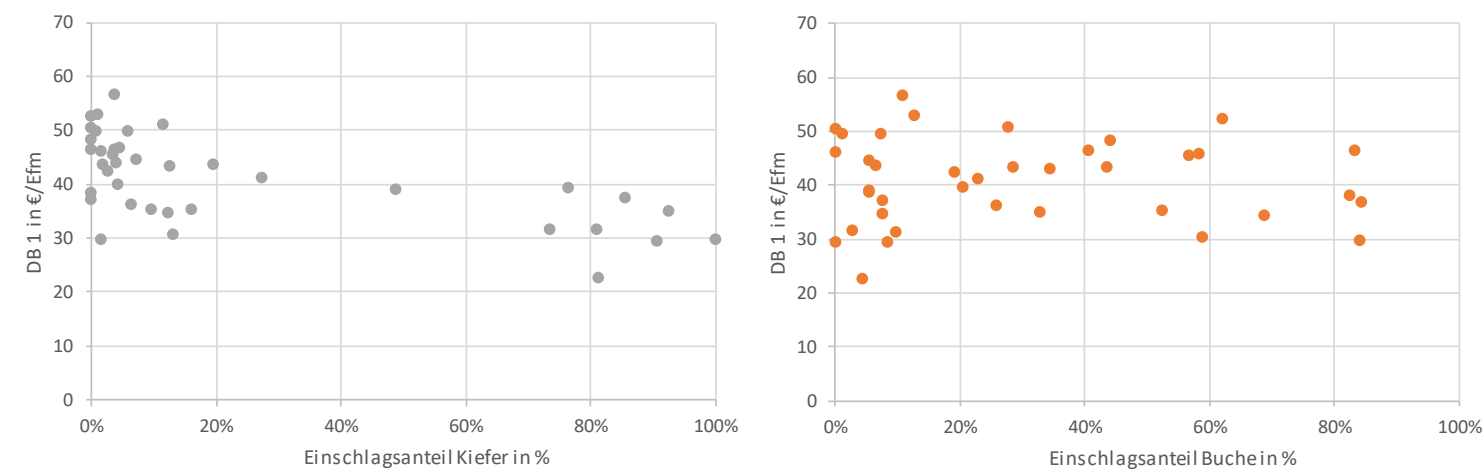

Abbildung 19: Zusammenhang von Einschlagsanteil der Baumartengruppen Buche und Kiefer und erzieltem Deckungsbeitrag I (erntekostenfreier Holzerlös), $n=38$

Dieser Einfluss der Fichte auf den wirtschaftlichen Erfolg der Erwerbsforstbetriebe verdeutlicht einmal mehr das Spannungsfeld von Ökonomie und Ökologie, wenn im Rahmen von Vertragsnaturschutzmaßnahmen bspw. hochproduktive Nadelholzbestände in Laubholzbestände umgebaut werden.

\subsubsection{Ertragssituation Landwirtschaft}

Für die befragten Betriebe, die auch landwirtschaftliche Flächen bewirtschaften lässt sich aufgrund der geringen Anzahl sowie der heterogenen Ausgangslagen kein spezifischer, aussagekräftiger Betriebsvergleich erstellen. Nachfolgend werden daher kurz die zentralen Ergebnisse des Wirtschaftsjahres 2017 aus dem allgemeinen landwirtschaftlichen Betriebsvergleich der BB Göttingen GmbH dargestellt, zu dem die in dieser Arbeit betrachteten Betriebe mit ihren Zahlen ebenfalls beigetragen haben.

Auf naturaler Ebene haben die landwirtschaftlichen Betriebe im Wirtschaftsjahr 2017/18 eher schwach geerntet (s. Abbildung 20). Im Durchschnitt aller westdeutschen Betriebe lag der Winterweizen bei 8,3 t/ha, das entspricht dem geringsten Niveau seit dem WJ 2012/13. Der Winterraps lag bei lediglich 3,6 t/ha und somit auf dem Niveau des schwachen WJ 2011/12. Die geringe Steigerung der Naturalerträge im Getreide in den vergangenen Jahren wird vor allem auf mangelnden züchterischen Fortschritt und ackerbauliche Umsetzung zurückgeführt (Warnecke et al. 2019, S. 19). Die Zuckerrübe hat mit einem Zuckerertrag von 15,9 t/ha hingegen den höchsten Wert der gesamten Zeitreihe erreicht. Hier ist der züchterische Fortschritt klar erkennbar (Warnecke et al. 2019, S. 19). Im Wissen um die Erträge der Wirtschaftsjahre 2018/19 und 2019/20, die wie auch der Wald, durch die starke Dürre geprägt waren, kann das Wirtschaftsjahr 2017/18 retrospektiv noch als durchschnittlich betrachtet werden. 


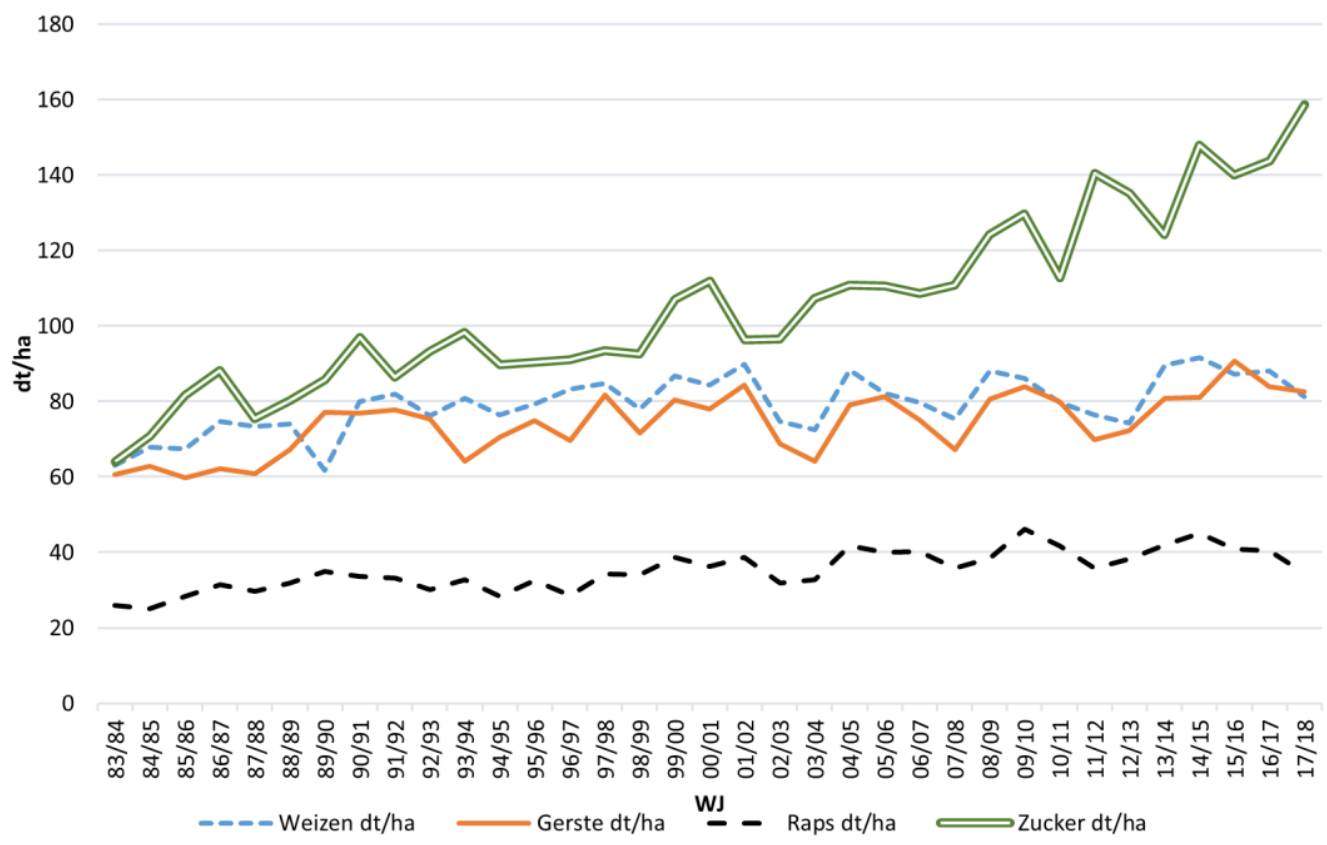

Abbildung 20: Entwicklung der Naturalerträge Landwirtschaft Deutschland West WJ 83/84 bis WJ 17/18, Quelle: BB Göttingen $\mathrm{GmbH}$

Zur Darstellung des wirtschaftlichen Erfolgs in der Landwirtschaft wird die Grundrente als Kenngröße verwendet. Sie beschreibt das Roheinkommen abzüglich Lohn- und Zinsansatz (Warnecke et al. 2019, S. 11).

Im Wirtschaftsjahr 2017/18 liegt die Grundrente in Westdeutschland bei $390 € /$ ha (s. Abbildung 21). Damit liegt sie deutlich unter dem von der BB Göttingen $\mathrm{GmbH}$ formulierten Ziel von $500 € /$ ha, die notwendig sind, um bei dem derzeit herrschenden Preisgefüge an den Bodenmärkten konkurrenzfähige Pachten zahlen zu können. Der Anteil, den die Direktzahlungen aus der 1. Säule der GAP ausmachen, beläuft sich auf 78 \% der Grundrente. Ohne diese Subventionen hätten die meisten Betriebe ein negatives kalkulatorisches Ergebnis ${ }^{24}$ erzielt (Warnecke et al. 2019, S. 12). Die Erträge aus Agrarumweltmaßnahmen werden in der Auswertung den Direktzahlungen zugeordnet. Ihre genaue Höhe ist nicht erfasst, jedoch haben sie dazu beigetragen, den Rückgang der Direktzahlungen aus der 1. Säule abzupuffern (Warnecke et al. 2019, S. 12).

\footnotetext{
${ }^{24}$ Das kalkulatorische Ergebnis setzt sich aus dem Roheinkommen abzüglich Lohn-, Zins- und Pachtansatz zusammen und ist das Ergebnis der Vollkostenrechnung. Es wird auch als Unternehmergewinn bezeichnet.
} 


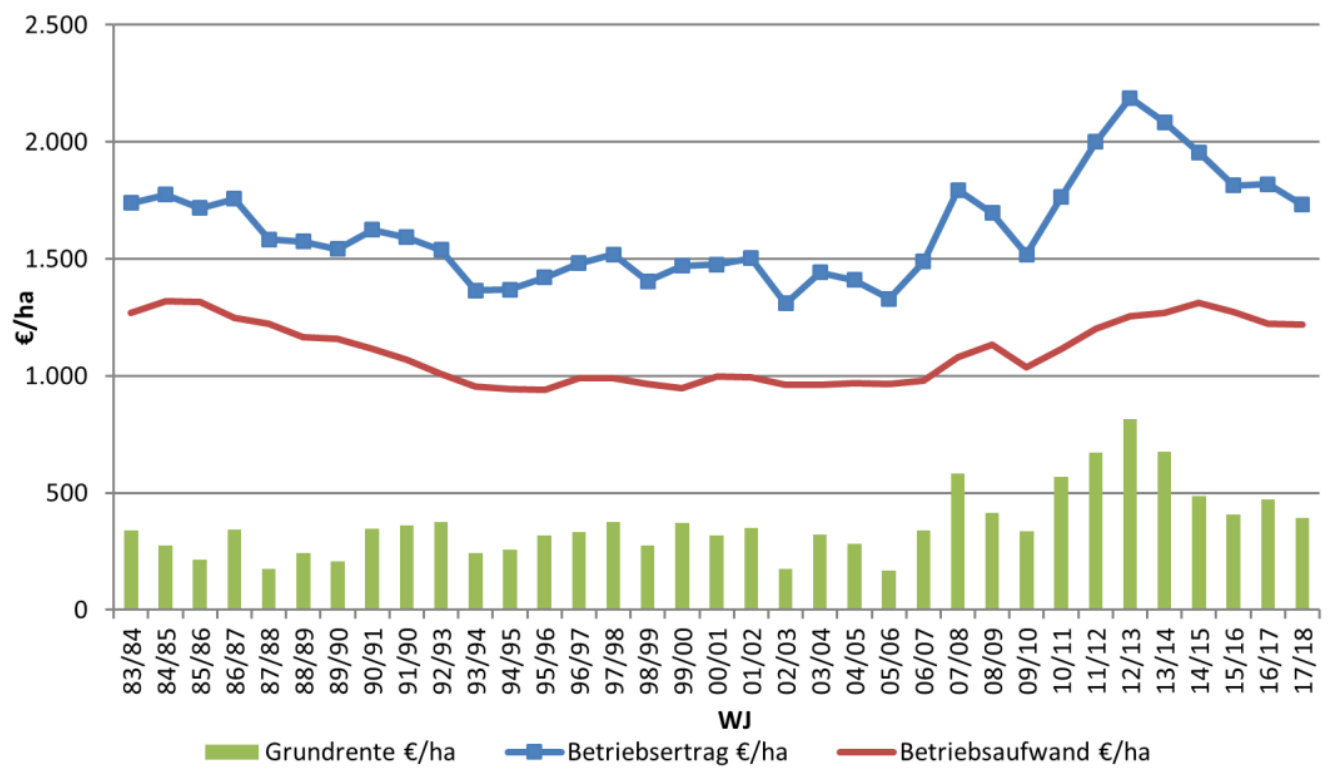

Abbildung 21: Entwicklung landwirtschaftlicher Betriebsertrag, Betriebsaufwand und Grundrente, Deutschland West WJ 83/84 bis 17/18, Quelle: BB Göttingen $\mathrm{GmbH}$

Zusammenfassend lässt sich feststellen, dass die Ertragssituation in der Landwirtschaft im Wirtschaftsjahr 2017/18 bereits angespannt war. Nach den wirtschaftlich sehr guten Jahren von 2010/11 bis 2013/14 hat eine deutliche Abschwächung sowohl durch die Naturalerträge als auch die Marktsituation stattgefunden. Die Direktzahlungen der Europäischen Union stellen einen wesentlichen Beitrag dar, ohne den viele Betriebe nicht auskömmlich wirtschaften könnten.

\subsubsection{Zielsysteme Waldeigentümer}

Im ersten Teil des Fragebogens wurden neben allgemeinen betrieblichen und persönlichen Daten der Befragten auch die aus Sicht des Eigentümers, Betriebsleiters oder Dienstleisters maßgeblichen betrieblichen Ziele abgefragt. Dabei wurde mit geschlossenen Fragen und vorgegebenen Antwortbegriffen gearbeitet, um eine Vergleichbarkeit der Betriebe zu ermöglichen.

Alle 40 auswertbaren Betriebsdatensätze haben diese Fragen beantwortet. Die Auswertung erfolgte mittels Profillinien für jeden einzelnen Betrieb (s. linker Teil der Abbildung 22), sowie dem daraus ergebenden arithmetischen Mittelwert mit Standardabweichung (s. rechter Teil der Abbildung 22). 


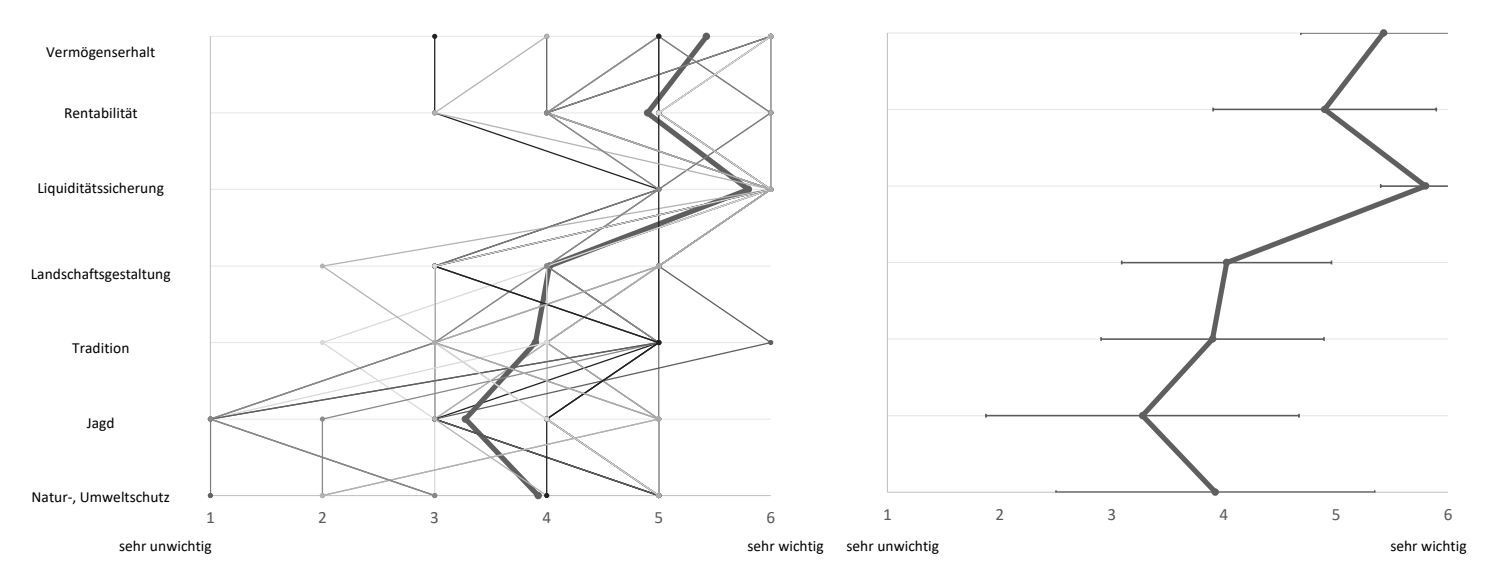

Abbildung 22: Zielsystem Betriebszweig Forstwirtschaft BB-Forstbetriebe $(n=40)$, zweite Darstellung nur Mittelwert mit Standardabweichung ${ }^{25}$

Für die Ziele im Betriebszweig Forstwirtschaft lassen sich zwei klare Schwerpunktziele identifizieren (s. Abbildung 22). An erster Stelle steht mit einer durchschnittlichen Gewichtung von 5,8 das Ziel der Liquiditätssicherung ${ }^{26}$, gefolgt vom Vermögenserhalt mit 5,5. Deutlich zu erkennen ist bei diesen beiden Zielen, dass die Antworten der Betriebe wenig streuen. Die Rentabilität als Betriebsziel folgt erst an dritter Stelle. Ziele wie Landschaftsgestaltung, Tradition, Jagd und Natur-, bzw. Umweltschutz stehen bei den befragten Betrieben an nachrangiger Stelle, die Einzelantworten variieren dabei auf der vollen Skalenbreite, insgesamt ergeben sich durchschnittliche Werte zwischen 3,3 und 3,7. Die Standardabweichung als Streuungsmaß der Antworten deutet, mit Ausnahme der Liquiditätssicherung als wichtigstes Ziel, auf keine weiteren übergeordneten Ziele hin.

Von den 40 befragten Betrieben verfügen 18 Betriebe zusätzlich über landwirtschaftliche Flächen, die sie bewirtschaften. Auch in diesem Betriebszweig steht der Vermögenserhalt als Betriebsziel an erster Stelle mit einem Mittelwert von 5,6, abweichend zum Forst gefolgt von der Rentabilität mit einem Wert von 5,4 (s. Abbildung 23). Die Liquiditätssicherung steht anders als im Forst erst an dritter Stelle. Landschaftsgestaltung wird gegenüber dem Forst (Wichtigkeit 3,8) ein etwas höherer Stellenwert beigemessen $(4,0)$. Tradition und Jagd hingegen spielen eine sehr untergeordnete Rolle, wohingegen dem Natur- und Umweltschutz ein deutlich höherer Stellenwert als im Forst beigemessen wird. Dieser zunächst überraschende Befund, dass Natur- und Umweltschutz im Bereich der Landwirtschaft ein höherer Stellenwert als im Forst beigemessen wird, kann gegebenenfalls dadurch erklärt werden, dass Natur- und Umweltschutzvorgaben die

\footnotetext{
${ }^{25}$ In den nachfolgenden Abbildungen wird aus Gründen der Übersichtlichkeit jeweils nur die rechte Darstellungsform mit Mittelwert und Standardabweichung gewählt.

${ }^{26}$ Die Liquiditätssicherung kann auch als Sparkassenfunktion für schlechte Zeiten beschrieben werden (vgl. Becker und Borchers 2000, S. 34). Sie äußert sich in der Praxis auch durch oftmals hohe Vorräte in eigentlich hiebsreifen Beständen, deren Nutzung aus ökonomischer Perspektive vorteilhaft wäre (vgl. Johansson und Löfgren 1985, S. 80).
} 
landwirtschaftliche Produktion stärker dominieren als im Waldbereich, so dass die Betriebe die entsprechenden Vorgaben systematisch als Ziele verfolgen müssen, wollen sie beispielsweise nicht gegen pflanzenschutzrechtliche Vorgaben verstoßen und im Rahmen von Cross-Compliance-Vorgaben Fördermittel verlieren. Auch ist anzunehmen, dass das Antwortverhalten der Befragten gesellschaftliche Ansprüche an die Landwirtschaft antizipiert und dem Natur- und Umweltschutz daher ein höherer Stellenwert als im Wald beigemessen wird, wo er in der Wahrnehmung der Teilnehmer teilweise noch im „Kielwasser“27 automatisch mit umgesetzt wird.

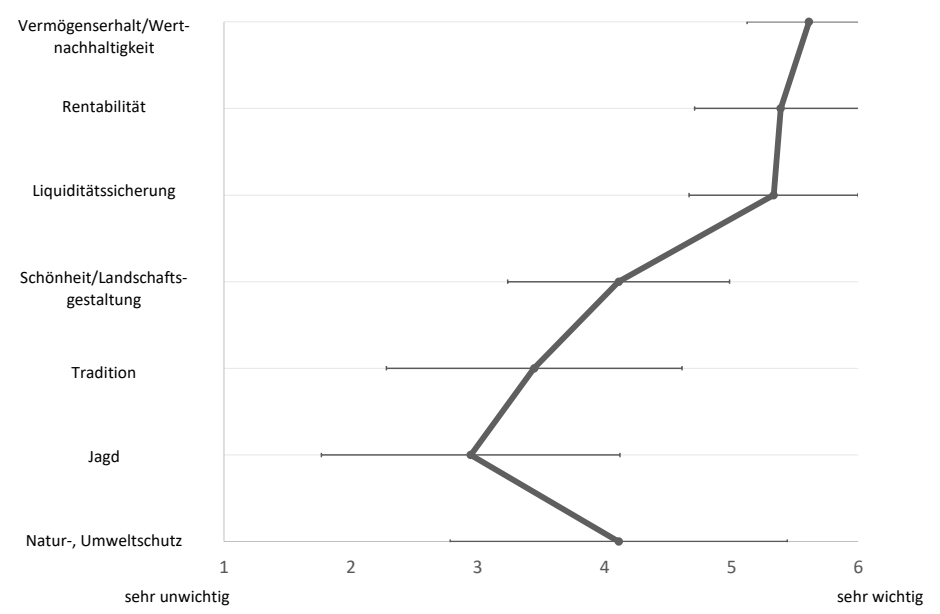

Abbildung 23: Zielsystem Betriebszweig Landwirtschaft BB-Gemischtbetriebe ( $n=18)$

Insgesamt zeigt sich eine klare ökonomische Ausrichtung der landwirtschaftlichen Ziele, vor der die eher als sozio-ökologisch zu bezeichnenden Ziele (wie Tradition, und Natur-/Umweltschutz) in den Hintergrund treten.

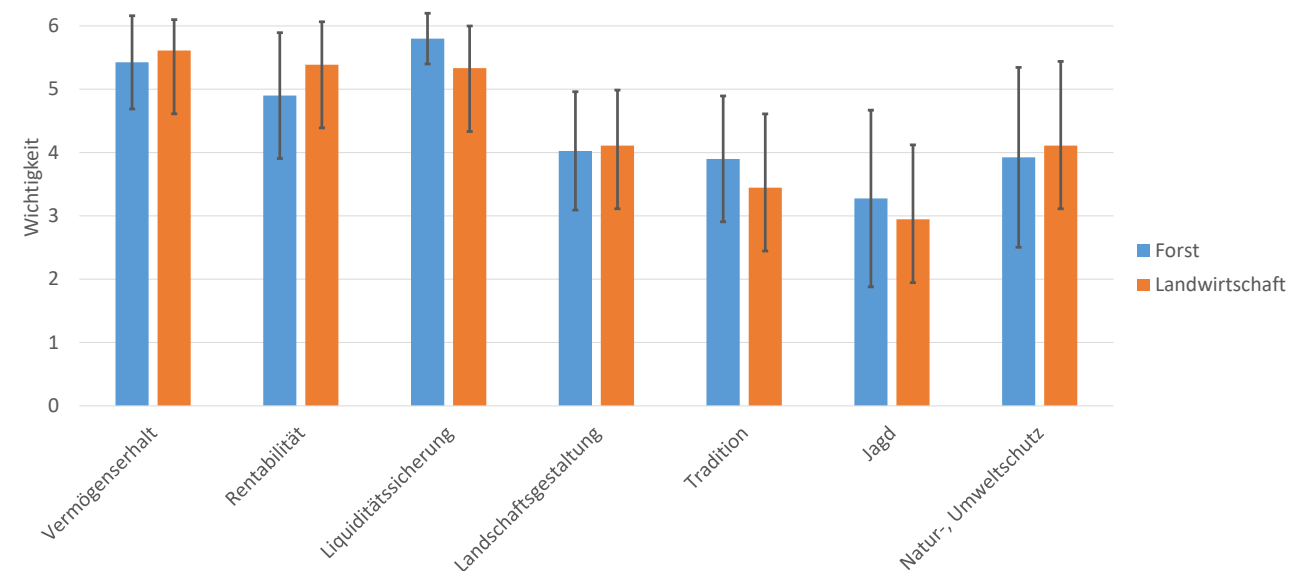

Abbildung 24: Alternative Darstellung Vergleich der land- und forstwirtschaftlichen Ziele der befragten BB-Betriebe, Mittelwerte der Kategorien inkl. Standardabweichung

${ }^{27}$ Die Kielwassertheorie von Rupf (1960, S.22) unterstellt eine Erbringung der sonstigen Waldfunktionen automatisch ohne weiteres Handeln im Rahmen einer normalen Waldbewirtschaftung (Thoroe 2009, S. 6), mittlerweile widerlegt und abgelöst vom Konzept der Multifunktionalen Forstwirtschaft (vgl. Suda und Pukall 2014, S. 333). 


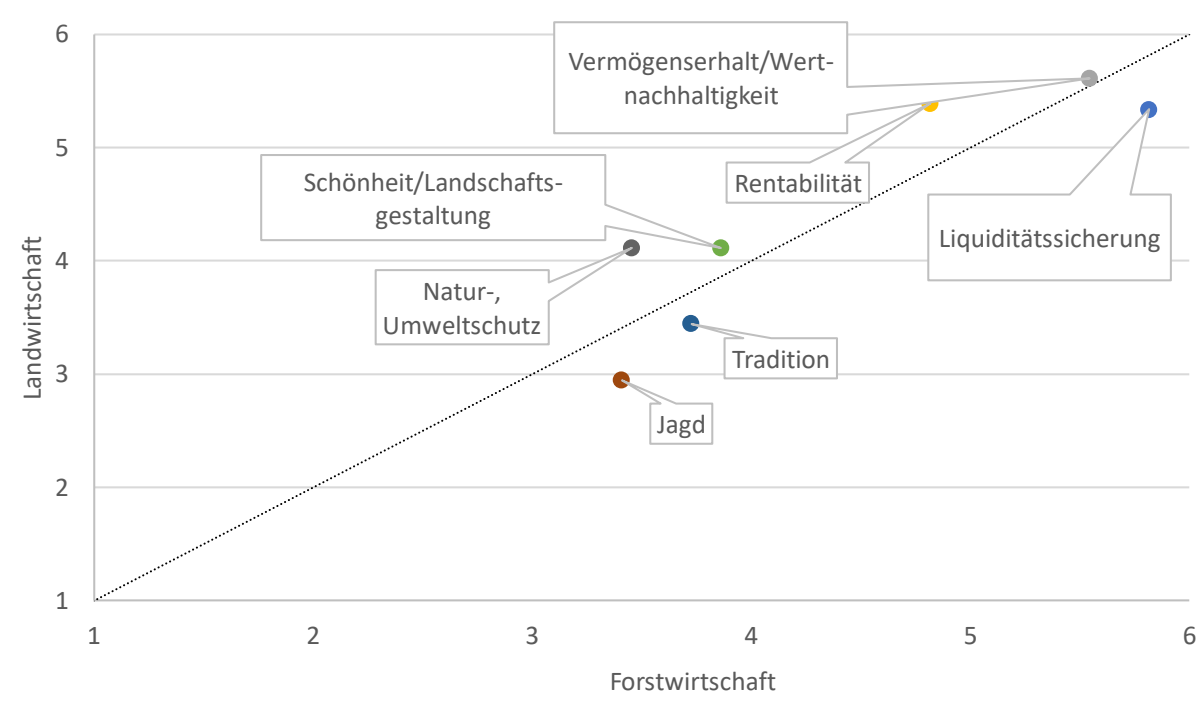

Abbildung 25: Vergleich der land- und forstwirtschaftlichen Ziele der befragten BB-Betriebe, Mittelwerte der Kategorien

Im Vergleich der Zielsysteme der Land- und Forstwirtschaft ist zunächst festzustellen, dass die Zielgewichte sehr ähnlich strukturiert sind. Die Unterschiede sind marginal. In Abbildung 25 wird zum Vergleich der Zielsysteme die Darstellung über der Winkelhalbierenden gewählt. Elemente oberhalb der Winkelhalbierenden besitzen in der Landwirtschaft eine höhere Wichtigkeit, Elemente unterhalb der Winkelhalbierenden haben einen höheren Stellenwert in der Forstwirtschaft. Die Aspekte Liquiditätssicherung, Tradition und Jagd sind im Wald wichtiger als in der Landwirtschaft, den Aspekten Vermögenserhalt, Rentabilität, Landschaftsgestaltung und Naturschutz wurde in der landwirtschaftlichen Zielsetzung ein höherer Wert beigemessen.

Im zweiten Teil des Fragebogens wurden die Betriebe zu ihren Erfahrungen, Hintergründen und Absichten von umgesetzten Naturschutzmaßnahmen gegen Entgelt im Wald und Agrarumweltmaßnahmen in der Landwirtschaft befragt. Nicht alle befragten Betriebe haben dabei solche Maßnahmen bislang umgesetzt. 25 der 40 Betriebe (63\%) verfügen über direkte, betriebliche Erfahrungen mit Naturschutzmaßnahmen gegen Entgelt im Wald. Dennoch liegt dieser Wert vermutlich deutlich über dem bundesweiten Durchschnitt von privaten Forstbetrieben, die Vertragsnaturschutz durchgeführt haben. Leider liegen hierzu keine konkreten Zahlen vor und konnten auch im WaVerNa-Projekt nicht erhoben werden (s. 2.3). In der Landwirtschaft hingegen haben 17 der 18 gemischten Betriebe (94\%) in der Vergangenheit oder aktuell Agrarumweltmaßnahmen umgesetzt, während es bspw. in Niedersachsen im gleichen Zeitraum nur 39 \% der Betriebe waren (vgl. Niedersächsisches Ministerium für Ernährung, Landwirtschaft und Verbraucherschutz 2019). Beide Zahlen weisen jedoch deutlich auf die Akzeptanz und Attraktivität, bzw. die erfolgreiche Umsetzung dieser Maßnahmen hin. 
Befragt nach den Zielen, die die Betriebe mit Naturschutz gegen Entgelt im Wald erreichen wollten, zeigt sich gegenüber den betrieblichen Zielen ein deutlich weniger stark differenziertes Bild (s. Abbildung 26). Mit leichtem Vorsprung wichtigstes Ziel ist nach Angabe der Befragten die Verbesserung des Natur- \& Umweltschutzes mit einem Mittelwert von 4,3. Danach folgt das Ziel der Rentabilität durch Inwertsetzung ökonomisch bislang nur geringwertiger Flächen oder Objekte mit einem Wert von 4,0, danach folgt die Förderung der gesellschaftlichen Akzeptanz. Betriebliche Diversifizierung, Landschaftsgestaltung und Tradition spielen die geringste Rolle bei der Entscheidung zur Umsetzung solcher Maßnahmen. Auffällig in den Zielsetzungen zur Teilnahme an Naturschutzmaßnahmen gegen Entgelt im Wald ist die große Varianz zwischen den Betrieben, hier ausgedrückt durch die Standardabweichung.

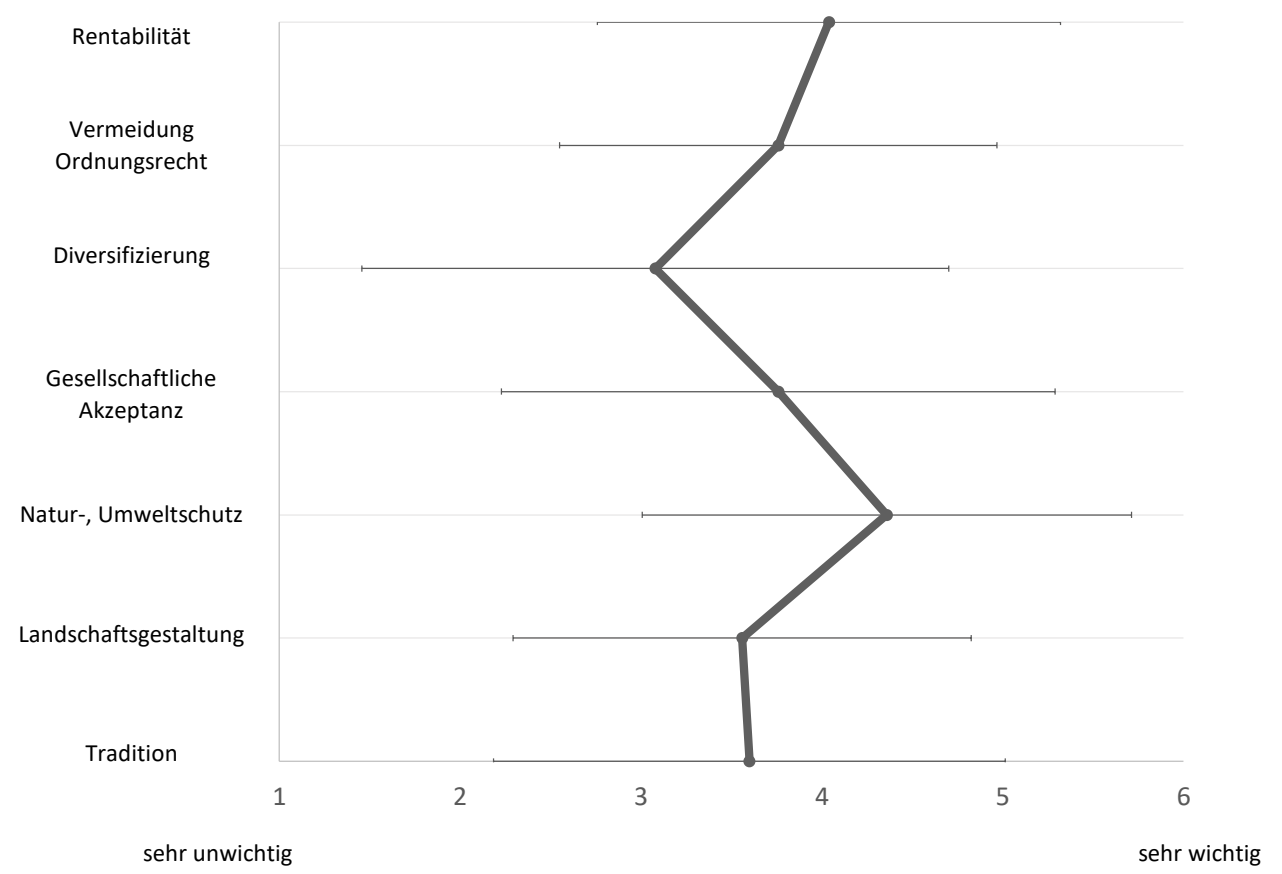

Abbildung 26: Zielsystem Teilnahme an Naturschutzmaßnahmen gegen Entgelt im Wald BB-Forstbetriebe ( $n=25)$

Auch in den landwirtschaftlichen Betrieben ist das Hauptziel, das mit Agrarumweltmaßnahmen verfolgt wird, die Förderung des Natur- und Umweltschutzes $(4,5)$, gefolgt von der Steigerung der gesellschaftlichen Akzeptanz $(4,4)$. Erst an dritter Stelle folgt die Rentabilität mit 4,2. Diversifizierung und Tradition spielen in der Landwirtschaft wie im Forst eine nachgeordnete Rolle. 


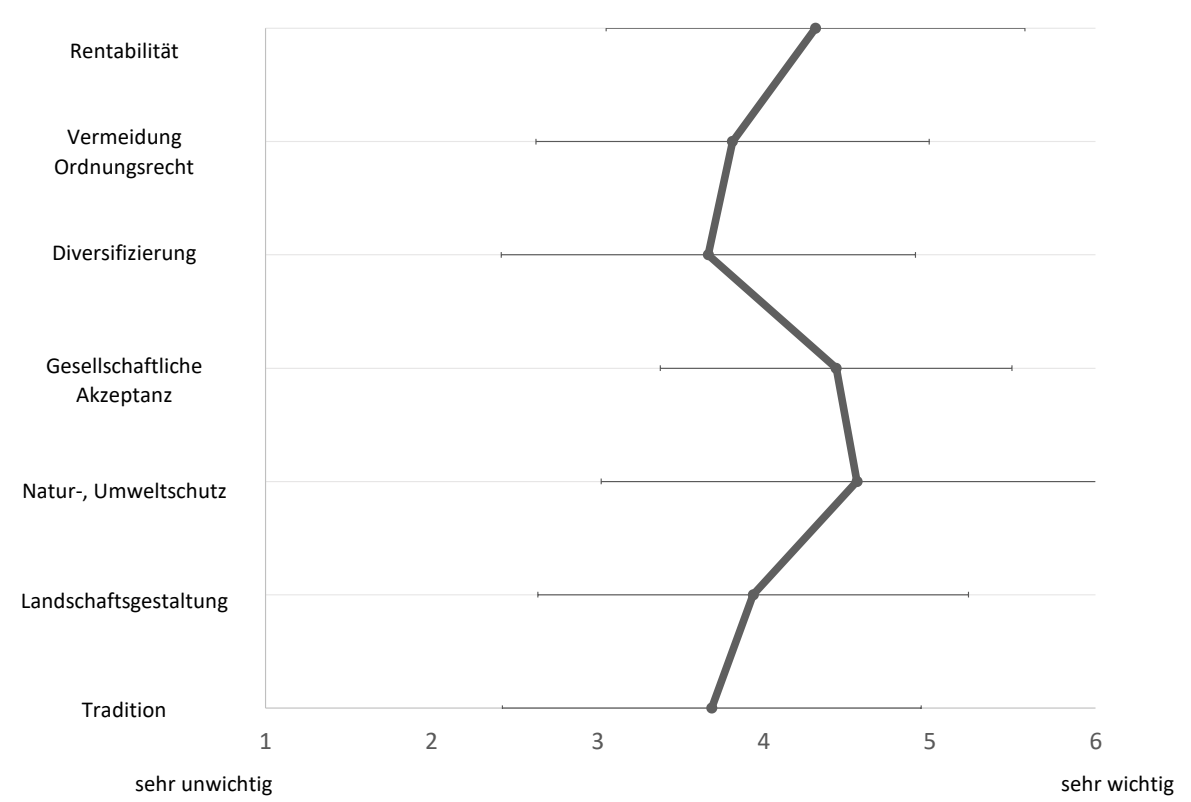

Abbildung 27: Zielsystem Teilnahme an Vertragsnaturschutzmaßnahmen Landwirtschaft BB-Gemischtbetriebe $(n=17)$

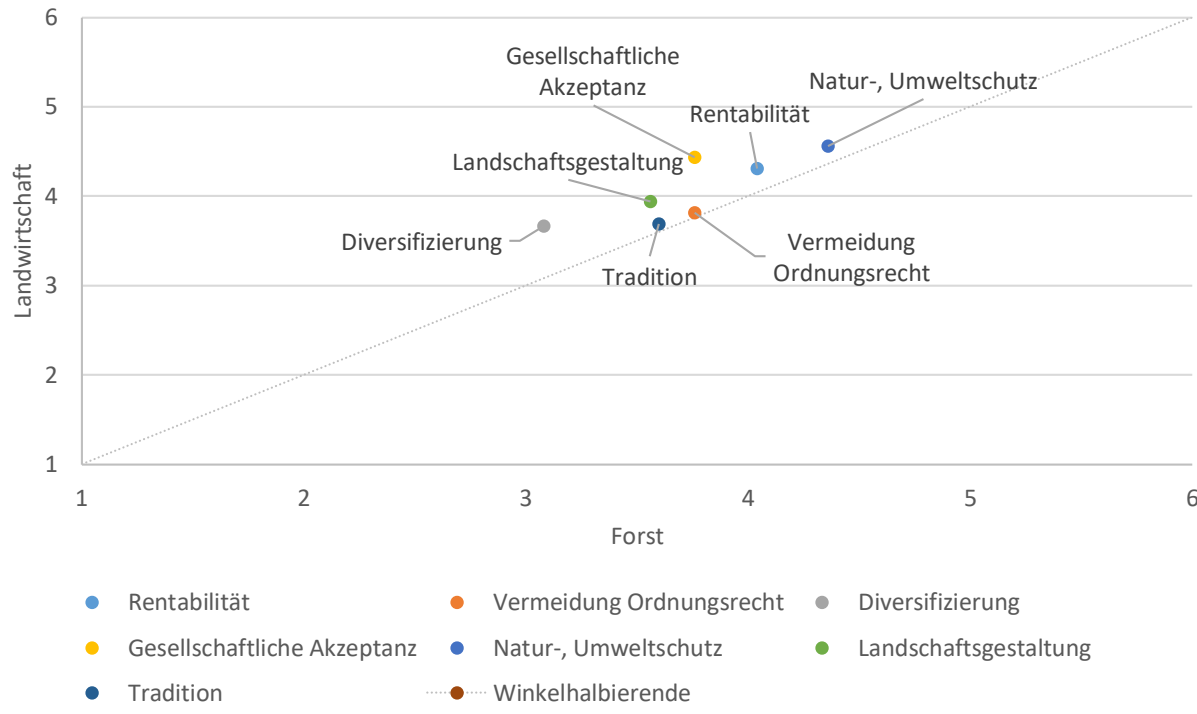

Abbildung 28: Vergleich der Ziele der landwirtschaftlichen und der forstwirtschaftlichen Betriebe für die Teilnahme an Vertragsnaturschutzmaßnahmen, Mittelwerte der Kategorien

\subsubsection{Beurteilung Vertragsnaturschutz in der Land- und Forstwirtschaft}

Die BB-Betriebe wurden um eine Bewertung ihrer umgesetzten Vertragsnaturschutzmaßnahmen in der Land- und der Forstwirtschaft hinsichtlich der identifizierten Erfolgsfaktoren (s. Franz et al. 2018b) gebeten. Wie in Abbildung 29 zu erkennen, sind die vier Erfolgsfaktoren in der Landwirtschaft durchweg besser bewertet worden. Insgesamt bestärkt diese Bild die Einschätzung, dass Agrarumweltmaßnahmen zumindest aus der Sicht der Betriebe als „Erfolgsmodell“ angesehen werden (s. Güthler et al. 2005). Auch die Standardabweichungen sind im Bereich der 
Landwirtschaft deutlich geringer, was darauf hindeutet, dass hier einheitlichere Erfahrungen vorliegen. Die großen Streuungen der Einschätzungen im Forstbereich hingegen deuten darauf hin, dass beim Instrument Vertragsnaturschutz im Wald sehr unterschiedliche Erfahrungen gemacht wurden, was nicht zuletzt damit zusammenhängen dürfte, dass es hier eine große Regelungsvielfalt gibt und sich das System noch nicht fest etabliert hat. Bei allen identifizierten Erfolgsfaktoren liegt der Forstbereich offensichtlich deutlich zurück, der größte Unterschied zeigt sich bei der Kontinuität der Finanzierung, die bereits mehrfach als größtes Hemmnis für einen erfolgreichen Vertragsnaturschutz im Wald angesprochen wurde. Auch die Standardabweichung als Streuungsmaß der gegebenen Antworten ist im Forst wesentlich größer als in der Landwirtschaft.

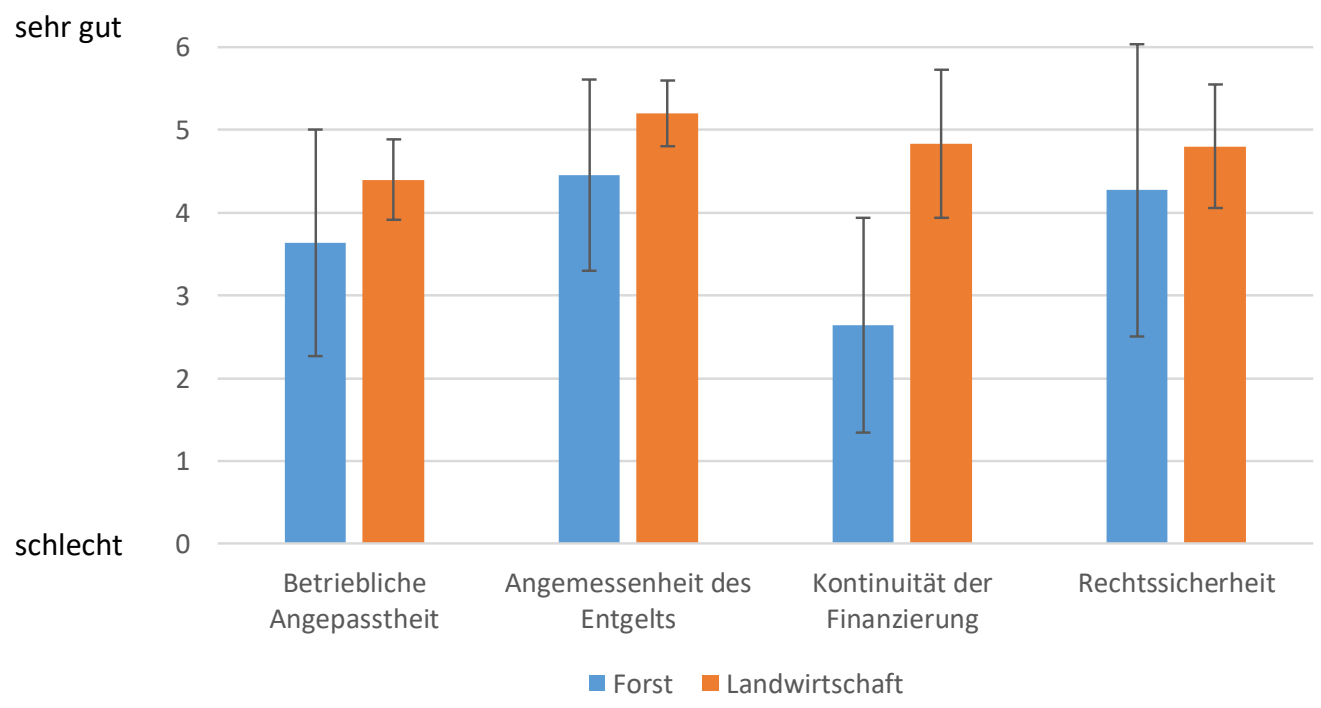

Abbildung 29: Beurteilung Vertragsnaturschutz in der Land- und Forstwirtschaft, Mittelwerte inkl. Standardabweichung BB-Betriebe mit umgesetzten Maßnahmen ( $n=19)$

Wie bereits im WaVerNa-Projekts beschrieben (Güthler et al. 2005 in Seintsch et al. 2018) und in der Literatur anderweitig veröffentlicht (Bundesministerium für Ernährung und Landwirtschaft 2014a, S. 127) gilt Vertragsnaturschutz in der Landwirtschaft als Erfolgsmodell. Die Befragung der BB-Forstbetriebe, die gleichzeitig auch landwirtschaftliche Betriebszweige haben, eröffnet die seltene Möglichkeit bei einem Experten in Form des Eigentümers oder Betriebsleiters ein ähnliches Thema in zwei Wirkungsfeldern zu analysieren. Ziel der Befragung war es, wie oben bereits ausgeführt, auch eine vertiefende Analyse des landwirtschaftlichen Vertragsnaturschutzes durchzuführen, um daraus Hinweise auf Umsetzungsmöglichkeiten für den Vertragsnaturschutz im Wald zu gewinnen. Methodisch wurde dabei wie schon in Kapitel 3.2 beschrieben, vorgegangen. 


\subsubsection{Ergebnisse}

\subsubsection{Umgesetzte Maßnahmen}

Die Agrarumweltmaßnahmen in der Landwirtschaft sind die durch die Länder konkretisierten ELER-Fördermaßnahmen aus der zweiten Säule der Europäischen Union. Aus der föderalen Konkretisierung resultieren bei grundsätzlich gleichen oder ähnlichen Maßnahmen teilweise sehr unterschiedliche Förderangebote, Umsetzungs- und Kontrollvorschriften in den Bundesländern, da diese die Fördermaßnahmen ihren regionalen Gegebenheiten und Bedürfnissen angepasst haben.

18 der 40 befragten BB-Betriebe sind sogenannte Mischbetriebe, die neben Forst auch Landwirtschaft betreiben. Als Agrarumweltmaßnahmen oder ähnliche Vertragsnaturschutzmaßnahmen wurden in diesen Betrieben vorrangig Ackerrandstreifen, bzw. Blühstreifen angelegt oder Grünlandflächen extensiviert. Auch der Anbau von Zwischenfrüchten zwischen zwei Hauptkulturen auf Ackerflächen war eine häufig genannte Maßnahme. ${ }^{28}$ Diese Maßnahmen resultieren aus der Teilnahme an ELER-Landesprogrammen wie bspw. PFEIL in Niedersachsen oder HALM in Hessen. Neben diesen allgemeinen Angeboten haben einige Betriebe auch regionale Vertragsnaturschutzmaßnahmen umgesetzt, bei denen Einzelmaßnahmen aufgrund ihrer ökologischen Wertigkeit mit örtlichen Vertragspartnern vereinbart wurden. Als Kleinprojekte bezeichnet, fallen hierunter Maßnahmen zum Amphibienschutz an Teich-, Laich- und Fließgewässern, die Schafhaltung zur Pflege extensiven Grünlands oder auch die Renaturierung von Gewässern. Überregionale Projekte, die nicht durch europäische, Bundes- oder Landesmittel finanziert wurden, wurden auch in der Landwirtschaft nicht umgesetzt. In der Gruppe der untersuchten Betriebe spielten Personen des privaten Rechts (Einzelpersonen, Stiftungen etc.) als Nachfrage nach Naturschutzleistungen also keine Rolle.

\subsubsection{Umsetzungszeiträume}

Die Umsetzung der Vertragsnaturschutzmaßnahmen in der Landwirtschaft belaufen sich bei den teilnehmenden Betrieben maximal auf fünf bis sieben Jahre der ELER-Förderperioden, teilweise

\footnotetext{
${ }^{28}$ Mit Einführung der Greeningprämie in den Direktzahlungen der aktuellen Förderperiode der Europäischen Union sind konventionell wirtschaftende Landwirte verpflichtet, $5 \%$ ihrer Ackerflächen als ökologische Vorrangflächen auszuweisen (Brachen, Hecken, Randstreifen, etc.) (Europäisches Parlament; Rat der Europäischen Union 2013b). Agrarumweltmaßnahmen wie Blühstreifen oder Zwischenfrüchte können mit einem Reduktionsfaktor auf die Erfüllung der Greeningauflagen angerechnet werden (vgl. Europäisches Parlament; Rat der Europäischen Union 2013a). In diesem Fall werden die ELER-Förderbeträge aufgrund des Doppelförderungsverbots gekürzt, da die Gelder der Greeningprämie aus der 1. Säule der GAP fließen.
} 
sind es auch nur einjährige Laufzeiten, die sich mit dem annuellen Zyklus der Bodenbewirtschaftung decken. Dennoch bleiben viele Agrarumweltmaßnahmen auch über mehrere Förderperioden gleich oder sehr ähnlich (bspw. Blühstreifen), so dass lange Erfahrungshorizonte vorliegen.

\subsubsection{Anteil Betriebsertrag}

Für die befragten Betriebe nehmen die Zahlungen aus ELER-Förderprogrammen oder anderen Vertragsnaturschutzmaßnahmen gemessen am Gesamtbetriebsertrag eine untergeordnete Rolle ein. Sie liegen zwischen 0,5\% und $8 \%$. Wichtiger ist den Befragten der Ausgleich ihrer Aufwendungen und vor allem die Inwertsetzung von landwirtschaftlichen Grenzertragsstandorten, auf denen die Zahlungen für Vertragsnaturschutzmaßnahmen typischerweise die landwirtschaftlichen Deckungsbeiträge übersteigen. Neben dem Ersatz der betrieblichen Kosten ist in den Zahlungen regelmäßig auch ein Anreizfaktor enthalten, so dass die Maßnahmen zum Betriebsgewinn beitragen.

\subsubsection{Beurteilung Agrarumweltmaßnahmen}

Die Vertragsnaturschutzmaßnahmen der Landwirtschaft werden von den teilnehmenden Betrieben überwiegend positiv beurteilt. Die Maßnahmen sind gut in die Betriebsabläufe integrierbar, in ihren Auswirkungen überschaubar, die ökologische Wirksamkeit wird als hoch eingeschätzt und die Zahlungen werden als angemessen betrachtet. Probleme bereiten vielerorts die gesetzten Fristen, die einer ordnungsgemäßen Bewirtschaftung aufgrund zu früher Aussaattermine oder zu später Nutzungsmöglichkeiten entgegenstehen. Die Aussagen der befragten BB-Betriebe decken sich mit der vorliegenden Literatur zu Agrarumweltmaßnahmen (vgl. Niens und Marggraf 2010, Oppermann 2013).

\subsubsection{Gründe für Nichtteilnahme an Vertragsnaturschutz im Wald}

Im zweiten Teil des Fragebogens wurden neben den Zielsetzungen für die Teilnahme an Naturschutz gegen Entgelt im Wald auch etwaige Gründe im Falle der Nichtteilnahme abgefragt. Von den 40 befragten Betrieben, haben 15 in der Vergangenheit keine Naturschutzmaßnahmen gegen Entgelt im Wald umgesetzt. Die Gründe dafür lassen sich anhand der Antworten in vier Kategorien ohne Zuordnung einer Rangfolge einteilen. 
1. Im entsprechenden Bundesland waren aus Sicht der Befragten keine Angebote öffentlicher Institutionen (Förderprogramme, ÖPP ${ }^{29}$, o.ä.) bekannt.

2. Die angebotenen Maßnahmen sind in der Planung und Durchführung zu komplex und die Verpflichtung zur Einhaltung der Vorgaben ist für die Betriebe ein zu hohes Risiko.

3. Die Zahlungshöhen sind zu gering, Zahlungs- und Verpflichtungszeiträume sind nicht kongruent.

4. Es besteht die Sorge durch die temporäre (freiwillige) Verpflichtung Zustände zu schaffen, die aufgrund des Artenschutzes nach BNatSchG die weitere Bewirtschaftung der Flächen erschweren.

\subsubsection{Betriebliche Bedeutung des Entgelts für Vertragsnaturschutz im Wald}

Der Anteil des Betriebsertrags, den die Vertragsentgelte aus den umgesetzten Maßnahmen ausmachen, ist in den befragten Forstbetrieben sehr gering. Sie liegen nach Aussage der Befragten zwischen $1 \%$ bis maximal $10 \%$, wobei dieser Höchstwert aus einem eigens mit dem örtlichen Landkreis ausgehandelten Naturschutzvertrag resultiert und somit einen Einzelfall darstellt. Die Mehrheit der Betriebe, die überregional angebotene Programme umgesetzt haben, erzielen damit einen Betriebsertragsanteil von ca. 3 \%. Dieser Betrag deckt sich mit den zuvor dargestellten Fördererträgen aus der Erhebung der Betriebsvergleichsdaten.

\subsubsection{Umsetzungsprobleme des Vertragsnaturschutzes im Wald}

Die Betriebe, die Naturschutz gegen Entgelt im Wald in der Vergangenheit durchgeführt haben, wurden nach Problemen bei der Umsetzung der Maßnahmen befragt. Betriebe, die bislang keine Maßnahmen durchgeführt haben, wurden nach den Hinderungsgründen befragt. Die beschriebenen Probleme lassen eine Kategorisierung in drei Gruppen zu. Wörtliche Zitate aus den Interviews werden dabei kursiv dargestellt:

1. Anbahnung

Bei den Betrieben, die bereits Erfahrungen mit Vertragsnaturschutz im Wald gemacht haben, wurde als häufigstes Problem während der Planung und Durchführung der „überbordende Verwaltungsaufwand" als Herausforderung genannt. Die Prozesse der Antragstellung waren oft

\footnotetext{
29 Öffentlich-private-Partnerschaften (englisch PPP): Als ÖPPs werden vertragliche Vereinbarungen zwischen der öffentlichen Hand und privaten Unternehmen zur effizienten Erreichung gemeinwohlorientierter Ziele bezeichnet. Während der private Partner die Leistungserstellung übernimmt, achtet die öffentliche Hand auf die Einhaltung der Ziele. ÖPP sind typischerweise einem Miet- oder Pachtverhältnis ähnlich (Gerstlberger und Schneider 2008).
} 
„zeitraubend" und „unübersichtlich“.Erschwerende Faktoren waren eine nicht vorhandene Deckungsgleichheit von naturschutzfachlichen oder verwaltungsorganisatorischen und forstlichen Planungseinheiten. ${ }^{30}$

\section{Dokumentation}

Weiterhin wurden die Dokumentationspflichten als enorm zeitaufwendig beschrieben, so dass der „Abwicklungsaufwand nicht im Verhältnis zum Ertrag“ stünde. Durch teilweise jährlich verpflichtende Neueinmessungen der Naturschutzobjekte und erneuten Antragstellungen in Verbindung mit unregelmäßigen Änderungen der Förderbedingungen werden die Vertragsnaturschutzmaßnahmen für die Betriebe so aufwändig, dass eine Durchführung „nur unter ständiger Zuhilfenahme von Praktikanten möglich ist".

\section{Kommunikation}

Die dritte große Problemkategorie aus der Befragung der BB-Betriebe lässt sich unter dem Begriff der Kommunikation zwischen den beteiligten Partien subsumieren. Lässt der Vertragsnaturschutz dem Grunde nach ein Verhandeln auf Augenhöhe erwarten, ist die Wahrnehmung der Betriebe eine deutlich andere. So wurde in der Befragung von "unklaren Regelungen“ gesprochen, bei denen auch die Behördenmitarbeiter nicht über die notwendigen Informationen zur Umsetzung verfügten. In anderen Fällen konnten Anpassungen der Maßnahmen nicht im Dialog geklärt werden, sondern mussten „ausschließlich auf gerichtlicher Ebene" durchgesetzt werden. Insgesamt kann in den Befragungen eine stark gestörte Kommunikation zwischen den Parteien festgestellt werden, die Betriebe empfinden sich oftmals als Bittsteller ohne Mitspracherecht und kritisieren eine Maßnahmengestaltung ausschließlich aus naturschutzfachlicher Sicht ohne Berücksichtigung land- und forstwirtschaftlicher Belange.

\subsubsection{Stärken von Vertragsnaturschutz im Wald}

Die Betriebsleiter und Eigentümer wurden zu den Stärken von Naturschutz gegen Entgelt aus ihrer Sicht befragt. Hintergrund der Frage war, neben den bereits vorangehend dargestellten Problemen auch die Chancen und Möglichkeiten freiwillig ökologischer Maßnahmen zu ermitteln.

\footnotetext{
${ }^{30}$ Stichwort: Waldblock vs. Abteilung
} 
Die Einschätzungen der Befragten gingen dabei stark auseinander. Die gegebenen Antworten lassen sich auf Grundlage gewählter Schlüsselwörter in vier Haltungen gruppieren:

1. Die erste Gruppe der Befragten sieht in Naturschutz gegen Entgelt im Wald die Stärke Naturschutz an seiner wirksamsten Stelle umzusetzen, da aufgrund der Kenntnisse des Betriebsleiters oder Eigentümers und seiner Zustimmung die Maßnahmen dort umgesetzt werden können, wo die ökologische Aufwertung besteht und die betriebliche Integration möglich ist (14 Nennungen, Schlüsselwörter: angepasst, wirksam, Augenhöhe, spezielles Wissen des Eigentümers).

2. Die zweite Gruppe der Befragten sieht vor allem die Steigerung der gesellschaftlichen Akzeptanz als maßgeblichen Vorteil des freiwilligen, bezahlten Naturschutzes (8 Nennungen, Schlüsselwörter: Gesellschaft, Akzeptanz, öffentliche Leistung).

3. Die dritte Gruppe hat eine eher pessimistischere Grundhaltung, die in dem freiwilligen Naturschutz gegen Entgelt im Wald die größte Stärke in der Vermeidung ordnungsrechtlicher Auflagen sieht. Aus Sicht der Befragten seien freiwillige Maßnahmen dem Ordnungsrecht jederzeit vorzuziehen und böten die Chance, zusätzlichen, verpflichtenden Auflagen vorzubeugen (6 Nennungen, Schlüsselwörter: Vermeidung, Ordnungsrecht, Zwang, freiwillig).

4. Die letzte Gruppe der Befragten sieht entweder keine Stärken oder steht dem Naturschutz gegen Entgelt grundsätzlich ablehnend gegenüber (12 Nennungen, Schlüsselwörter: nicht bekannt, Ablehnung, kein Mehrwert, Forst ist auch ohne Vertragsnaturschutz naturnah). Nach der ersten Gruppe stellen diese Betriebe den zweitgrößten Anteil dar.

\subsubsection{Analyse der Erfolgsfaktoren und Möglichkeiten der Optimierung}

In der Befragung der BB-Forstbetriebe wurden die vier im WaVerNa-Projekt identifizierten Erfolgsfaktoren a) forstbetriebliche Angepasstheit ${ }^{31}$ b) finanzielle Angemessenheit ${ }^{32}$ c) Kontinuität der Finanzierung und d) Rechtssicherheit auch einer detaillierteren, inhaltlichen Analyse unterzogen. In der BB-Befragung wurden diese validiert, inhaltlich vertieft und untersucht, welche Verbesserungsmöglichkeiten aus Sicht der Betriebe bestehen. Wörtliche Zitate aus den Antworten der befragten Experten sind zur Dokumentation kursiv dargestellt.

\footnotetext{
${ }^{31}$ In 2.4.2.2 als Flexibilität bezeichnet

32 In 2.4.2.2 als Fairness bezeichnet
} 


\section{Forstbetriebliche Angepasstheit}

In Kapitel 2.4.2.2 ist die forstbetriebliche Angepasstheit von Naturschutzmaßnahmen gegen Entgelt identifiziert worden. Die befragten BB-Forstbetriebe betrachten eine an forstlichen Gegebenheiten orientierte Gestaltung von Maßnahmen mit „Bezug zu forstlichen Planungsgrößen“ als unerlässlich für die grundsätzliche Umsetzbarkeit. In der Vergangenheit angebotene Maßnahmen, die „zu starr" also aufgrund ihrer Kleinstrukturiertheit, technisch nicht umsetzbaren Anforderungen oder zu hohem Arbeitsaufwand für Betriebe unattraktiv waren, wurden aufgrund des Mangels der forstbetrieblichen Angepasstheit nicht umgesetzt.

Als Verbesserungsmöglichkeit wurde die Anlehnung an etablierte forstliche Handlungen und Planungsebenen empfohlen, wie es auch in der Landwirtschaft stattfindet. Die erfolgreichen Maßnahmen der Landwirtschaft sind in der handwerklichen Umsetzung der klassischen ackerbaulichen Tätigkeit sehr ähnlich (z. B. Anlage von Blühstreifen). Weiterhin bedarf es „einfacher Maßnahmen“ mit einer höheren „Flexibilität", um den örtlichen Gegebenheiten angepasst werden zu können.

\section{Finanzielle Angemessenheit}

Die Frage nach der finanziellen Angemessenheit wurde von den befragten Betrieben sehr unterschiedlich beantwortet. Grundsätzlich hängt ein angemessenes Entgelt sehr eng mit dem nachfolgenden Erfolgsfaktor, der Kontinuität der Finanzierung zusammen. Aus Sicht der Betriebe ist ein Ausgleich des Minderertrags nur dann ausreichend, wenn sich die Maßnahme nahezu ohne Änderung der betrieblichen Abläufe integrieren lässt. Sobald ein erhöhter Kontroll- und Verwaltungsaufwand oder sogar eine grundsätzliche Änderung der betrieblichen Abläufe notwendig ist, bedarf es eines „echten Anreizfaktors", um den Betrieb zu motivieren, dieses Wagnis einzugehen. Ein ausschließlicher Ausgleich der direkten Kosten der Maßnahmen (inkl. der Opportunitätskosten) reicht i.d.R. nicht aus. Die „ökologische Wirkung“ ist für die Befragten keine ausreichende Motivation. Ein weiterer vorgebrachter Verbesserungsvorschlag bestand in einer „steuerlichen Berücksichtigung der Vertragsnaturschutzzahlungen für die 6b-Rücklage“"133.

\footnotetext{
${ }^{33}$ Der $\S 6 b$ EstG ermöglicht die (steuerfreie) Übertragung stiller Reserven beim Verkauf von Grund und Boden, Aufwuchs, Gebäuden (und Binnenschiffen) auf ein Ersatzwirtschaftsgut zumeist gleicher Form innerhalb eines fünfjährigen Zeitraums. Vertragsnaturschutzzahlungen sollten somit dieser Rücklage zuführbar sein, um sie ebenfalls steuerfrei investieren zu können.
} 


\section{Kontinuität der Finanzierung}

Neben der Höhe des Entgelts für die vertraglich vereinbarte Maßnahme ist für die befragten Betriebe die Sicherung der langfristigen Finanzierung von größerer Bedeutung, denn die naturschutzbedingten Maßnahmen haben im Forstbereich i.d.R. sehr langfristige Folgen und „kurzfristige Förderprogramme nützen im Wald nichts". Mit Blick auf die Kontinuität der Finanzierung spielt aber auch ein anderer Aspekt eine Rolle. Bereits die Auseinandersetzung mit dem Thema Vertragsnaturschutz erfordert von den Forstbetrieben in der Vorbereitungsphase viel Einsatz (Prüfung der fachlichen und institutionellen Grundlagen, Prüfung der betrieblichen Möglichkeiten und Änderung der betrieblichen Planung, Kalkulation und Rechtsberatung, Anpassung der betrieblichen Prozesse etc.). Es entstehen mithin erhebliche betriebliche Fixkosten, die sich nur dann lohnen, wenn Vertragsnaturschutz als ein langfristiges Geschäftsfeld wahrgenommen wird und zu erwarten ist, dass sich die Rahmenbedingungen dafür in der Zukunft nicht fundamental verändern werden. Ein großes Manko von Vertragsnaturschutzmaßnahmen in der Vergangenheit war auch die Diskrepanz zwischen Verpflichtungszeitraum (Zweckbindungsfrist) und Finanzierungszeitraum (Sachverständigenrat für Umweltfragen und Wissenschaftlicher Beirat für Waldpolitik 2017, S. 6). Oftmals wurden Maßnahmen angeboten, deren Wirkungsdauer die Zahlungszeiträume deutlich überschritten und die für die Betriebe dadurch sehr unattraktiv waren. Dieses Problem mangelnder Kontinuität, oftmals den mittelfristigen Finanzplanungen oder dem Haushaltsvorbehalt der Parlamente geschuldet, ist für die BB-Betriebe nach wie vor ungelöst und macht somit Naturschutzmaßnahmen gegen Entgelt, bspw. im Vergleich zu Ausgleichs- und Ersatzmaßnahmen, deren Finanzierung durch den Eingriffsverursacher gesetzlich gesichert ist, unattraktiv.

Die Betriebe sehen für dieses Problem jedoch auch Lösungsmöglichkeiten, die diese Problematik entschärfen können. Wie bereits in vielen Bundesländern bei der Maßnahme „Erhalt von Altund Habitatbäumen" umgesetzt (vgl. Bayrisches Staatsministerium für Ernährung, Landwirtschaft und Forsten 2014) und im Bewertungskonzept für Einzelbäume methodisch beschrieben (s. Kapitel 4.3.3) können „kapitalisierte Einmalzahlungen“ vor dem Hintergrund der unsicheren Finanzierungsquellen insbesondere für Einzelobjekte die Akzeptanz fördern, da für den Waldbesitzer das finanzielle Risiko abgemindert wird. Der ökologisch wertvolle Baum wird dem Waldbesitzer somit "abgekauft" und von diesem für den Verpflichtungszeitraum oder sogar dauerhaft bis zum natürlichen Zerfall erhalten. Methodisch und für den Besitzer psychologisch entspricht dieses Vorgehen dem klassischen Holzverkauf auf dem Stock, ist somit nah an den gewohnten forstlichen Handlungsweisen. Dies wird unter anderem auch ein Grund sein, 
weshalb der Erhalt von Alt- und Habitatbäumen bislang die häufigste Maßnahme des Vertragsnaturschutzes im Wald ist.

Eine von den Waldbesitzern vorgeschlagene Alternative besteht in „periodischen Zahlungen für erhaltene Einzelobjekte wie Alt- und Habitatbäume“. So lange ein ökologisch wertvoller Baum vorhanden ist, erhält der Waldbesitzer zu festzulegenden Zeitpunkten entsprechende Zahlungen. Dabei besteht die Erwartung der Befragten, dass somit auch der Verwaltungs- und Kontrollaufwand für beide Seiten zu reduzieren wäre.

Ein weiterer Lösungsansatz besteht aus der Sicht der befragten Akteure in der Einrichtung von haushalts- oder förderperiodenunabhängigen Finanzierungsquellen. Die "Schaffung von Fonds oder Stiftungen“, die aus ihren Erträgen oder dem Stiftungskapital Naturschutzmaßnahmen finanzieren können, erscheint den Befragten als geeignete Lösung, um angebotene Naturschutzmaßnahmen gegen Entgelt unter dem Aspekt der Kontinuität der Finanzierung umzusetzen.

4. Rechtssicherheit

Neben den organisatorischen und finanziellen Faktoren ist für die Betriebe auch die Rechtssicherheit ihres Handelns von hoher Bedeutung. Bei der Umsetzung von Naturschutzmaßnahmen gegen Entgelt spielen zwei Aspekte eine wesentliche Rolle.

a) Rechtssichere Umsetzung/Klarheit der Handlungen

Zunächst müssen Maßnahmen in ihren Anforderungen so beschrieben sein, dass für die Betriebe eine rechtssichere Umsetzung möglich ist. Unklarheiten gefährden sowohl den naturschutzfachlichen als auch den finanziellen Erfolg. Es bedarf eindeutiger Regelungen, die „nicht jedes Jahr geändert werden".

Besonders für die befragten BB-Betriebe, die über land- und forstwirtschaftliche Betriebszweige verfügen, war die Sorge vor Cross-Compliance-relevanten Verstößen sehr groß. Die Aussicht nennenswerte Teile der "landwirtschaftlichen Direktzahlungen einzubüßen“, weil Naturschutzmaßnahmen gegen Entgelt nicht rechtskonform umgesetzt wurden, indem Maßnahmen fehlerhaft umgesetzt oder Dokumentationspflichten nicht erfüllt werden, hält die Befragten von einer Teilnahme ab.

Weiterhin bestehen Unsicherheiten hinsichtlich des Erhalts „absterbender Baumriesen und der Verkehrssicherung". Gerade die häufig anzutreffende Maßnahme des Alt- und Habitatbaumerhalts bedarf klarer vertraglicher Regelungen hinsichtlich der Verkehrssicherungspflicht. In den 
Programmen der Bundesländer finden sich teilweise Regelungen, die förderunschädliche Eingriffe in einem solchen Fall ermöglichen (vgl. Bayrisches Staatsministerium für Ernährung, Landwirtschaft und Forsten 2014). Alternativ wäre eine "Übernahme der Verkehrssicherung durch die Naturschutzbehörde" denkbar.

b) Wiederaufnahme ursprüngliche Bewirtschaftung vs. Schaffung neuer naturschutzfachlicher Zustände

Der zweite wichtige Punkt wird in der Literatur unter dem sog. „Wiederaufnahmeprivileg“ beschrieben. § 14 Abs. 3 Nr. 1 BNatSchG wertet eine Wiederaufnahme der Bewirtschaftung, die zeitweise eingeschränkt oder unterbrochen war nicht als Eingriff, wenn sie auf Grund vertraglicher Vereinbarungen oder durch Teilnahme an öffentlichen Programmen zur Bewirtschaftungseinschränkung und innerhalb von zehn Jahren nach Ablauf dieser Beschränkungen vorgenommen wird. Nach Ablauf der Zweckbindungsfrist von Maßnahmen soll dabei dem Waldbesitzer die Fortsetzung der ursprünglichen Bewirtschaftung ermöglicht werden. Diese Wiederaufnahme forstlicher Tätigkeiten kann dabei im Widerspruch zu natur- und artenschutzrechtlichen Bestimmungen der Naturschutzgesetze von Bund und Ländern stehen ${ }^{34}$. Kommt es innerhalb der Vertragslaufzeit zur (ökologisch gewünschten) Schaffung natur- oder artenschutzrechtlich geschützter Zustände, so sind diese nach aktueller Rechtslage auch nach Ablauf der Frist zu erhalten. Damit ist die Wiederaufnahme der vorherigen Bewirtschaftung eingeschränkt und nicht unbeschränkt möglich. Es stehen sich der Schutz der natürlichen Lebensgrundlagen nach Art. 20 a GG als bedeutendes Staatsziel und das Grundrecht auf Eigentumsschutz widerstreitend gegenüber (Lutter und Paschke 2018, S. 29). Eine rechtliche Lösung hierfür besteht bislang nicht. Dieser Widerspruch führt in der Praxis zu einer starken Zurückhaltung der Waldbesitzer bei der Teilnahme an Naturschutzmaßnahmen gegen Entgelt. Dieser im WaVerNa-Projekt identifizierte mögliche Konflikt (s. Lutter und Paschke 2018, S. 29) zeigte sich auch in der Befragung der BBBetriebe besonders deutlich. Die ausschließlich erwerbswirtschaftlich orientierten Privatforstbetriebe messen diesem Faktor eine sehr hohe Bedeutung bei. Sie sehen den Gesetzgeber gefordert diesen Aspekt klar zu regeln, um eine rechtssichere Umsetzung von Naturschutzmaßnahmen gegen Entgelt zu ermöglichen. Gerade hinsichtlich der Rechtssicherheit ist aus Sicht der befragten Betriebe in der Vergangenheit „viel Porzellan zerschlagen worden“. Verstärkt wurde diese Wahrnehmung durch die als massiv wahrgenommenen Eingriffe in das Eigentum durch die Natura 2000-Schutzgebietsausweisungen der vergangenen Jahre. Die in den Interviews deutlich

\footnotetext{
${ }^{34} \mathrm{Vgl}$. BNatSch G § 44
} 
zum Vorschein gekommene Frustration der Betriebsleiter und Eigentümer gipfelte in der überspitzen Formulierung „rechtsstaatliches Handeln muss wieder verlässlich werden“.

\subsubsection{Bezugsobjekt der Vertragsnaturschutzmaßnahmen}

In vielen Programmen für Naturschutz gegen Entgelt im Wald finden sich ähnliche Maßnahmentypen. Dabei sind, neben der Schaffung neuer ökologisch wertvoller Zustände, die erhaltenden Maßnahmen nach ihrem Bezug in zwei Arten zu unterteilen. Erstens Maßnahmen, die sich auf den Erhalt einzelner Objekte beziehen, zweitens Maßnahmen, die einen Flächenbezug haben. In der Praxis (bspw. Bayrische Staatsforsten 2013) finden sich in Abhängigkeit des zu erreichenden Ziels Gründe für die jeweilige Gestaltungsform. ${ }^{35}$ Aus betrieblicher Sicht kann es einen erheblichen Unterschied machen, ob im Rahmen der regulären forstlichen Bewirtschaftung einzelne Objekte, wie z.B. Habitatbäume einem vereinbarten Schutz unterliegen oder ob ganze Flächen in der Planung zu berücksichtigen sind. Daher wurden die Teilnehmer der Befragung zur Vorteilhaftigkeit der beiden Bezugsarten aus ihrer Erfahrung befragt.

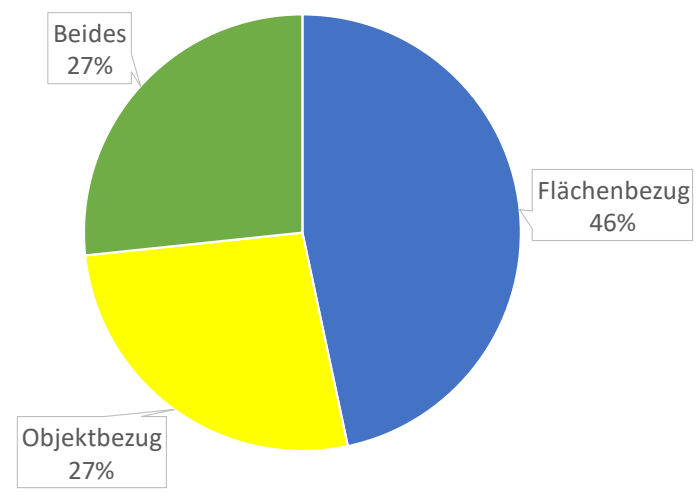

Abbildung 30: Bevorzugte Gestaltung von Vertragsnaturschutzmaßnahmen aus Sicht der BB-Betriebe, $n=27$

Etwa die Hälfte der Befragten (46 \%) zieht einen Flächenbezug für die Gestaltung von Naturschutzmaßnahmen gegen Entgelt vor. Sie begründen dies mit der gewohnten Planung forstlicher Maßnahmen auf Abteilungs-, Unterabteilungs- und Teilflächenebene. Dabei ist ihnen wichtig, dass die Naturschutzmaßnahmen ebenfalls auf diesen Ebenen geplant werden und umsetzbar sind. Maßgeblich ist dabei die Integrierbarkeit in die forstlichen Betriebsabläufe, die Erfassung

${ }^{35}$ Diese Unterteilung von Maßnahmen führte unter anderem auch zur Entwicklung der beiden in Kapitel 4 aktualisierten Bewertungskonzepte für Nutzungsbeschränkungen und für Einzelbäume. 
und Darstellung der Vertragsflächen in Karten und Betriebswerken sowie die klare Abgrenzbarkeit im Gelände. Eine Loslösung der Maßnahmen vom forstlichen Flächenbezug wäre aus ihrer Sicht kontraproduktiv.

Rund ein Viertel der Befragten (27\%) sehen hingegen den Objektbezug als vorteilhaft an. Insbesondere, wie bislang auch in vielen Programmen umgesetzt, könne der Alt- und Habitatbaumerhalt nur mit einem objektbezogenen Ansatz erfolgreich umgesetzt werden. Die Konzentration auf einzelne Objekte ermöglicht die konkrete Fixierung der vertraglichen Pflichten (Baumerhalt), die Bestimmung eines angemessenen Preises für die erbrachte Leistung und die Fortsetzung der Bewirtschaftung der umliegenden Bäume und Flächen. Auch sei mittlerweile durch satellitengestützte Ortungssysteme eine hinreichend präzise Einmessung der Vertragsobjekte möglich.

Ein weiteres Viertel der Gruppe (27\%) spricht sich für eine flexible Anwendung der Bezugsart in Abhängigkeit des zu erreichenden Ziels aus. Je nachdem, ob der ökologische Wert in einer Fläche oder einem Einzelobjekt liegt, sollten die vertraglichen Vereinbarungen dies berücksichtigen.

\subsubsection{Best-Practice-Beispiele im Wald \& Lösungen aus betrieblicher Sicht}

In der Konzeption des Fragebogens wurde davon ausgegangen, dass die befragten Betriebe, aufgrund ihrer Tätigkeiten und ihres Interesses im Bereich des Naturschutzes gegen Entgelt einen zumindest regionalen Überblick über das Engagement anderer Betriebe in diesem Bereich haben. Die Frage nach Best-Practice-Beispielen zielt auf die Identifikation solcher „Vorbilder" ab.

Es zeigte sich bei der Beantwortung jedoch, dass keine konkreten anderen Betriebe bekannt waren oder benannt werden konnten, die als Vorbilder herangezogen werden könnten. Umgekehrt wurden in der Beantwortung dieser Frage Maßnahmen oder Programme genannt, die teils aus eigener Erfahrung, teils aus konzeptionellen Gründen als Vorlage für eine Neugestaltung von Vertragsnaturschutzinstrumenten dienen könnten. Der Übergang zur nachfolgenden Frage nach aus forstlicher Sicht attraktiven Lösungen für die Gestaltung neuer Vertragsnaturschutzinstrumente war fließend.

Besonderes Augenmerk lag dabei auf den Ausgleichs- und Ersatzmaßnahmen (A\&E-Maßnahmen), unabhängig davon ob als projektbezogene Einzelmaßnahme oder in Form von Ökopunkten in einer Konto- oder Poollösung umgesetzt. Die klaren Regelungen in Bundesländern mit Kompensationsverordnungen, die sichere Mittelverfügbarkeit durch die Eingriffsverursacher und die Höhe der zu erzielenden Einnahmen, lassen diese Maßnahmen für die Betriebe sehr attraktiv erscheinen. Auch die Betriebe, die bereits Ausgleichs- und Ersatzmaßnahmen umgesetzt haben, sind von der Vorteilhaftigkeit dieses Systems überzeugt. 
A\&E-Maßnahmen wie standortgerechte Bestockungen oder die Renaturierung von Bachläufen sind technisch nah am forstlichen Handeln und für die Betriebe in Art und Umfang überschaubar. Einzig die Dauer unterscheidet sich deutlich von temporären Vertragsnaturschutzmaßnahmen, da Ausgleichs- und Ersatzmaßnahmen in der Regel bis zum Ende des zugeordneten Eingriffs bestehen müssen, was in der Praxis einer dauerhaften Bewirtschaftungsänderung entspricht. Das in Abhängigkeit der regionalen Märkte gezahlte Entgelt kompensiert diesen Nachteil aber offenbar ausreichend. Die technische Umsetzbarkeit korrespondiert stark mit der Überzeugung der Betriebe, dass Naturschutzmaßnahmen mit dem allgemeinen betrieblichen Handeln vereinbar sein müssen. Dazu zählt auch eine innerbetrieblich flexible Umsetzungsmöglichkeit. Hinzu kommt eine gesicherte Finanzierung durch den Eingriffsverursacher und in den meisten Bundesländern eine nachvollziehbare Wertermittlung der ökologischen Aufwertungen durch die Maßnahmen. Differenziert betrachtet wurde dabei die Vermarktungsfähigkeit von Ökopunkten aus vorweggenommenen Bevorratungen im Rahmen von Ökokonten oder -pools. Die Vorteilhaftigkeit solcher Ansätze ist stark abhängig von der räumlichen Lage des Betriebs, der allgemeinen Bautätigkeit in der Region und Kooperationsbereitschaft der örtlichen Naturschutzbehörden als Vermittler.

\subsection{Zwischenfazit}

Die vorangegangenen Ausführungen zeigen auf, dass, neben anderen Hemmnissen, insbesondere die Angemessenheit des Entgelts für viele Betriebe regelmäßig ein Problem darstellt. Um einen Beitrag zur erfolgreichen Umsetzung von Vertragsnaturschutz im Wald zu leisten, werden nachfolgend bestehende forstökonomische Bewertungskonzepte einer Aktualisierung und Weiterentwicklung unterzogen, um einerseits die Relevanz dieses Aspekts zu verdeutlichen und andererseits den beteiligten Akteuren ein verbessertes Handwerkszeug für die Ermittlung angemessener Entgelte bereitzustellen. 


\section{Aktualisierung und Weiterentwicklung von forstlichen Bewer- tungskonzepten}

\section{1.Ökonomische Auswirkungen durch Bewirtschaftungsänderungen}

Bei der Umsetzung von Naturschutzmaßnahmen gegen Entgelt ist davon auszugehen, dass den Forstbetrieben regelmäßig Mehraufwendungen und Mindererträge entstehen (Seintsch et al. 2018). Die Mehraufwendungen resultieren vorrangig aus aktiv durchzuführenden Handlungen auf operativer Ebene. Dies können Kosten investiver Art bspw. für die Begründung von Beständen mit ökologisch gewünschten Baumarten sein, aber auch die aktive Schaffung von Totholz oder die Wiedervernässung ehemaliger Moorflächen sind denkbare Handlungen, deren Durchführung den Forstbetrieben Aufwendungen verursachen. Für diese beschreib- und quantifizierbaren Projekte kann die Aufwandshöhe im Einzelfall für gewöhnlich ermittelt werden (Blomberg und Möhring 2018).

Aufwändiger ist die Ermittlung von Mindererträgen aus der vertraglich vereinbarten Bewirtschaftungsänderung. Es hat sich als zweckmäßig erwiesen, bei der ökonomischen Bewertung hier zwei verschiedene Bezugsgrößen zu unterscheiden: Bei flächigen Bewirtschaftungsänderungen werden zu erwartende flächige Mindererträge mittels Annuitätendifferenz der ökologisch angestrebten und der ökonomisch vorteilhaften Variante berechnet (Seintsch et al. 2019). Beim Erhalt einzelner Objekte im Wald (Alt- und Habitatbäume) ist es zweckmäßiger, ein Bewertungskonzept für Einzelbäume heranzuziehen (vgl. Möhring 2010).

Im Folgenden sollen zur Ermittlung der betriebswirtschaftlichen Auswirkungen von Naturschutzmaßnahmen gegen Entgelt mittels Modellrechnungen und anhand von Fallbeispielsanalysen beispielhaft die betriebswirtschaftlichen Nachteile (Mehraufwendungen und Mindererträge) bestimmt werden. Diese können als mindestens notwendige Ausgleichsbeträge verstanden werden, denn ohne entsprechenden finanziellen Ausgleich würde kein rationaler Entscheidungsträger solche Maßnahmen durchführen (vgl. Sagl 1995; Möhring et al. 2017).

Der in Kapitel 3.3.10 dargestellten Zweigleisigkeit zwischen einerseits flächen- und andererseits objektbezogenen Maßnahmen wird nachfolgend durch die Vorstellung und Aktualisierung zwei voneinander unabhängiger Bewertungskonzepte Rechnung getragen, die für den jeweiligen Bewertungsfall flächen- und objektbezogen angewendet werden können. 
Da die forstliche Produktion sich nicht - anders als in der Landwirtschaft - in einjährigen Perioden vollzieht, sondern viele Jahrzehnte umfasst und damit den Zeitpunkten der jeweiligen Zahlungen maßgebliche Bedeutung beikommt, bedarf es zur Quantifizierung der betriebswirtschaftlichen Auswirkungen von Nutzungsänderungen finanzmathematisch korrekter, auf Grundlage der in der Praxis vorhandenen Informationen anwendbarer Modelle, die die originären Zahlungsströme verschiedener Handlungsalternativen miteinander vergleichbar machen. Die Arbeiten im WaVerNa-Projekt an denen der Autor dieser Arbeit maßgeblich beteiligt war, haben die Erforderlichkeit handhabbarer und praktikabler Bewertungskonzepte, die auch für spezielle Situationen anwendbar sind, erneut verdeutlicht.

Da die Märkte der Forst- und Holzwirtschaft ebenso wie die naturalen und technischen Bedingungen kontinuierlich Veränderungen unterworfen sind, ist eine regelmäßige Aktualisierung und Anpassung der bestehenden Modelle erforderlich. Im Zuge dieser Arbeit wurden Aktualisierungen und konzeptionelle Erweiterungen von Bewertungskonzepten, die bei der Bewertung von Naturschutzmaßnahmen gegen Entgelt Anwendung finden können, vorgenommen. Sie sollen nun vorgestellt werden.

\subsection{Methodik}

Nachfolgend werden zwei bestehende und in der Praxis bewährte Bewertungskonzepte in ihren modelltheoretischen Überlegungen vorgestellt, anschließend erfolgt die Aktualisierung und konzeptionelle Weiterentwicklung und abschließend werden exemplarisch mögliche Anwendungen auf verschiedene Bewertungsfälle der forstlichen Praxis beschrieben. Die Erläuterung der methodischen Grundlagen des jeweiligen Modells erfolgt an entsprechender Stelle.

\subsection{Forstliche Bewertungskonzepte}

\subsubsection{Aktualisierung des Bewertungskonzepts für forstliche Nutzungsbe- schränkungen}

Bereits im Jahr 2006 wurde im „Bewertungskonzept für forstliche Nutzungsbeschränkungen“, erschienen als Band 32 in der Schriftenreihe zur Forstökonomie des Sauerländer-Verlages (Möhring und Rüping 2006), das Ziel verfolgt, ein auf praktische Fälle leicht anwendbares, in sich geschlossenes Bewertungskonzept zu entwickeln, das auf dem in der betriebswirtschaftlichen Bewertungslehre dominierenden Ertragswertkonzept beruht. Das hier vorgestellte aktualisierte Bewertungskonzept baut ebenso wie jenes auf jährlichen Erfolgsziffern der Holzproduktion in Euro je Hektar auf, die im Sinne von Annuitäten, also finanzmathematisch korrekt ermittelten jährlichen Deckungsbeiträgen aus der forstlichen Produktion, berechnet werden. Gerade aus 
Kreisen der forstlichen Praxis wurde vielfach der Bedarf nach derartigen jährlichen Zahlen artikuliert, die auch relativ leicht interpretiert und mit anderen Größen - wie z.B. jährlichen Ausgleichszahlungen in der Landwirtschaft - verglichen werden können.

Die starken Veränderungen im Forstsektor des vergangenen Jahrzehnts haben eine Neuberechnung notwendig gemacht. Die Holzmärkte haben in der Zeit von 2006 bis 2015 deutliche Veränderungen erfahren. Dabei folgten die Holzpreise einer, aus Sicht der Forstbetriebe, überwiegend positiven Entwicklung. Andererseits haben jedoch auch spürbare Kostensteigerungen stattgefunden. Die Auswirkungen dieser Veränderungen auf die bekannten Kalkulationen darzustellen und eine Erweiterung um praxisrelevante Bewertungsansätze vorzunehmen, waren die Ziele dieser Aktualisierung.

Die Berechnungen beziehen sich auf den Zeitraum von 2013 bis 2015 und wurden im Jahr 2017 veröffentlicht (s. Möhring et al. 2017). Die zum Zeitpunkt der Finalisierung dieser Arbeit (Herbst 2020) vorherrschenden Marktverwerfungen durch Stürme, Dürren und Borkenkäfer der letzten Jahre finden keinen Eingang in die Bewertung.

\subsubsection{Grundlagen}

Es gibt in der Forstwirtschaft eine zunehmende Anzahl von Gründen wie z.B. den von Seiten des Waldbesitzes angestrebten Vertragsnaturschutz in FFH-Gebieten, die Bewirtschaftung in Naturschutz- oder Wasserschutzgebieten, baurechtliche Kompensationsmaßnahmen etc., die eine Abweichung von der betriebswirtschaftlich optimalen Waldbewirtschaftung erfordern und so zu einer Minderung des forstwirtschaftlichen Erfolges führen. Zu denken ist hier beispielsweise an typische Maßnahmen wie:

- $\quad$ Baumartenwechsel, z.B. naturnahes Laubholz statt Nadelholz.

- Vorzeitige Auflichtung und Ernte von Nadelholzbestockungen, um sie durch andere Baumarten zu ersetzen.

- $\quad$ Langfristigen Erhalt von Laubholzbestockungen über die eigentliche Hiebsreife hinaus.

- Erhalt von Alt- und Habitatbäumen bis hin zu ihrem teilweisen oder vollständigen Zerfall.

Hier stellt sich regelmäßig die Frage nach der betriebswirtschaftlichen Betroffenheit des Waldbesitzers. Im Sinne des Grenzpreiskonzeptes kann der Betrag ermittelt werden, der mindestens gezahlt werden müsste, damit sich der Waldbesitzer nicht schlechter stellt als ohne diese Maßnahme. Die Grenzpreisermittlung erfolgt üblicherweise nach dem Ertragswertprinzip (s. Moxter 1983, S. 9). Der so ermittelte Ertragsverlust stellt jedoch nur einen "Mindestpreis" dar. Er darf nicht verwechselt werden mit einem „angemessenen Preis“, denn ein angemessener Preis hat 
regelmäßig höher zu liegen als der eigentliche Ertragsverlust, sonst würde sich der Belastete nicht auf einen entsprechenden Vertrag einlassen (s. Franz 2017, S. 2). In diesem Zusammenhang spricht die Bewertungstheorie von dem sog. "Schiedspreis“. Dieser bezeichnet einen „fairen" Einigungspreis, dessen Zweck der faire Interessenausgleich zwischen potenziellem Käufer und potenziellem Verkäufer ist (s. Moxter 1983, S. 22).

Die Bewertung nach dem Ertragswertprinzip vergleicht regelmäßig das Ergebnis der zu bewertenden Alternative mit der planmäßigen Entwicklung, auch Referenzentwicklung genannt. Wird unterstellt, dass der Forstbetrieb eine erwerbswirtschaftliche Ausrichtung verfolgt, so stellt die planmäßige, erwerbswirtschaftlich ausgerichtete forstliche Bewirtschaftung die „Referenz", also das Bezugssystem, dar. Dabei kann man jedoch nicht grundsätzlich davon ausgehen, dass die Beibehaltung des „Status quo“ auf der Waldfläche als Referenz anzusehen ist. So kann z.B. auch ein Baumartenwechsel (wie bspw. Ersatz einer wenig produktiven Laubholzbestockung durch den Anbau von Douglasie oder Fichte) als Referenzentwicklung dienen. Die Referenzentwicklung muss jedoch betriebswirtschaftlich vorteilhaft sein und auch praktisch angestrebt werden.

\subsubsection{Ziel dieses Bewertungskonzeptes}

Das im Folgenden beschriebene Bewertungskonzept zeigt einen Weg auf, wie Ertragsverluste als Folge von Abweichungen von der betriebswirtschaftlich optimalen Waldbewirtschaftung vereinfachend ermittelt werden können. Dieses Bewertungskonzept steht im Zusammenhang mit der Vertragswaldbewirtschaftung (z.B. Vertragsnaturschutz im Wald), bei der freiwillig, befristete Vereinbarungen über Nutzungsänderungen zu schließen sind. Es wird ausdrücklich darauf hingewiesen, dass Veränderungen der Verkehrs- oder Beleihungswerte ${ }^{36}$, die bspw. durch eine ordnungsrechtliche Schutzgebietsausweisung entstehen können und im Rahmen der Enteignungsentschädigung ggf. auszugleichen sind, nicht durch das vorgeschlagene Bewertungskonzept abgedeckt werden. Die mit Hilfe dieses Bewertungskonzeptes ermittelten Beträge sollen vielmehr als betriebswirtschaftliche Argumentationshilfe für Ausgleichszahlungen bei freiwilligen forstlichen Nutzungsbeschränkungen dienen.

Der hier beschriebene Vorschlag beschränkt sich auf die Ertragsverluste, die im Forstbetrieb aus der Veränderung der langfristigen „biologischen Produktion“ entstehen. Außerplanmäßige Fol-

\footnotetext{
${ }^{36}$ Zu dieser Fragestellung sind dem Autor in der forstwissenschaftlichen Literatur keine Untersuchungen bekannt. Anhaltsweise können Wertabschläge der Landwirtschaft übernommen werden (vgl. Mährlein 2017).
} 
gewirkungen (wie z.B. Randschäden) werden hier außer Acht gelassen. Das gilt auch für mögliche Ertragsverluste, die aus der Veränderung der „technischen Produktion“ entstehen. Diese können jedoch meist recht einfach über die Veränderungen der Kosten bewertet werden.

\subsubsection{Methodisches Konzept des Bewertungsverfahrens}

Abweichend von dem in der forstlichen Bewertung als Standardverfahren dominierend auftretenden und formal anerkannten System der Kapitalwertermittlung wird sich im vorliegenden Bewertungskonzept der Annuität bedient. Die originär unregelmäßig auftretenden Zahlungsströme werden in derivate jährlich konstante, aber ökonomisch äquivalente Zahlungsströme überführt. Formal erfolgt die Überführung des Kapitalwerts in die Annuität mittels des Annuitäten- oder Wiedergewinnungsfaktors.

Die ermittelte Über- (oder Unterschuss-)-größe wird im Bewertungskonzept als „jährlicher Holzproduktionswert" bezeichnet, kann aber ebenfalls als waldbaulicher Deckungsbeitrag verstanden werden, da neben den Erntekosten auch Bestandesbegründungs- und -pflegekosten Berücksichtigung finden. Die Annuität berücksichtigt insofern die variablen, bestandesbezogenen Erträge und Kosten inkl. der Kapitalkosten für entscheidungserhebliche Zahlungen, ohne die betriebsindividuellen Allgemein- oder Fixkosten miteinzubeziehen (s. Formel 1).

Formel 1: Annuität der forstlichen Produktion einer Umtriebszeit, Quelle: Möhring et al. 2006

$$
a_{u}=\left(\frac{A_{u}}{(1+i)^{u}}+\sum_{a=1}^{u} \frac{D_{a}}{(1+i)^{a}}-c\right) \cdot \frac{i \cdot(1+i)^{u}}{(1+i)^{u}-1}
$$

\footnotetext{
$\mathrm{a}_{\mathrm{u}}$ : $\quad$ Annuität der forstlichen Produktion einer Umtriebszeit

$A_{u}: \quad$ Abtriebswert im Alter $u$

$\mathrm{D}_{\mathrm{a}}$ : Durchforstungserlös im Alter a

c: Kulturkosten

i: $\quad$ Zins
}

Neben der Ermittlung des durchschnittlichen „jährlichen Holzproduktionswerts“ einer gesamten Umtriebszeit ist auch die Ermittlung der Annuität kürzerer Zeiträume möglich (s. Formel 2). Damit schließt das Bewertungskonzept an das sequenzielle forstliche Entscheidungsproblem an, bei dem während der laufenden forstlichen Produktion stets die Vorteilhaftigkeit der Nutzung oder der weitere Erhalt des Bestands überprüft werden muss. 
Formel 2: Annuität der forstlichen Produktion kürzerer Zeiträume, Quelle: Möhring et al. 2006

$$
a_{n}=\left(\frac{A_{x+n}}{(1+i)^{n}}+\sum_{a=x}^{x+n} \frac{D_{a}}{(1+i)^{a-x}}-A_{x}\right) \times \frac{i \times(i+1)^{n}}{(1+i)^{n}-1}
$$

\author{
$a_{n}: \quad$ Annuität der forstlichen Produktion für kürzere Zeiträume \\ $A_{x}$ : $\quad$ Abtriebswert im Alter $x$ \\ $A_{x+n}: \quad$ Abtriebswert im Alter $x+n$ \\ $\mathrm{x}$ : Bewertungszeitpunkt \\ $\mathrm{n}$ : Periodendauer \\ $D_{a}: \quad$ Durchforstungserlöse zum Zeitpunkt a \\ i: $\quad$ Zins
}

\title{
4.3.1.4. Aktualisierung der Datenbasis
}

Die Aktualisierung der Datenbasis bezog sich insbesondere auf die Veränderung der ökonomischen Inputgrößen, die naturalen (ertragskundlichen) Modellgrundlagen wurden unverändert übernommen. Insgesamt wurden bei den Beispielsberechnungen folgende Daten zugrunde gelegt:

1. Ertragstafeln als Produktionsmodelle, wobei als Standard für die Zuwachs- und Vorratsentwicklung die mäßige Durchforstung gewählt wurde (vgl. Schober 1975). Es erfolgte eine Kalibrierung des Ertragstafeldurchmessers an die heutigen waldbaulichen Verhältnisse (vgl. Wollborn und Böckmann 1998).

2. Holzpreise entstammen der Waldbewertungsrichtlinie Nordrhein-Westfalen des Jahres 2015 (Waldbewertungsrichtlinie Nordrhein-Westfalen (WBR NRW) 2015). Hier wurde für die Holzpreise einheitlich die Wertziffer 3 (mittlere Verhältnisse) unterstellt. Es handelt sich um Holzerlöse (einschließlich 5,5 \% Umsatzsteuer), also Bruttoerlöse vor Abzug der Holzerntekosten (inkl. 19 \% Umsatzsteuer).

3. Holzerntekosten für Nadelholz über die gesamte Durchmesserspreite und für Laubholz bis zu einem Brusthöhendurchmesser (BHD) von $40 \mathrm{~cm}$ wurden ebenfalls aus der nordrheinwestfälischen Waldbewertungsrichtlinie 2015 (WBR NRW 2015) abgeleitet. Beim Laubholz wird ab BHD $40 \mathrm{~cm}$ die motormanuelle Aufarbeitung unterstellt und die entsprechenden Holzerntekosten der WBR 2015 des Landes Nordrhein-Westfalen verwendet.

4. Bestandesbegründungskosten entstammen der niedersächsischen Waldbewertungsrichtlinie; diese sind die durchschnittlichen Kosten bei mittleren Kulturbedingungen und 
Wertverhältnissen der Kulturkostenstufe 4. Sie beinhalten alle Kosten bis zur Sicherung der Kultur, inklusive eines Lohnnebenkostenansatzes (WBR Niedersachsen 2014, Tab. 13)

5. Da für die Bestandesbegründung oftmals Förderungen gezahlt werden oder Naturverjüngungen ohne aufwendige Pflanzungen gelingen, wurden die Berechnungen mit und ohne Berücksichtigung von Kulturkosten durchgeführt.

6. Für alle Baumarten wurden für das Alter 10 und 20 Jahre Läuterungsmaßnahmen mit Kosten von jeweils $250,-$ EUR/ha unterstellt.

7. Der Zinsfuß wurde auf 1,5\% fixiert, wobei es sich um einen Realzins handelt, da inflationsbedingte Preis- und Kostenveränderungen nicht berücksichtigt werden.

Im Rahmen der Berechnungen wurde die Konstanz all dieser Daten in der Zeit unterstellt.

\subsubsection{Berechnung von Werttabellen}

Auf der Basis dieser Zahlengrundlagen wurden für die Hauptbaumarten Eiche, Buche, Fichte, Douglasie und Kiefer die „jährlichen Holzproduktionswerte in Euro je Hektar“ ermittelt. Die Berechnungen erfolgten sowohl für die gesamte Produktionsdauer (Dauer der Betrachtungsperiode entspricht der Umtriebszeit), als auch für unterschiedlich alte Bestände mit jeweils unterschiedlicher Dauer der Betrachtungsperiode.

Die Ergebnisse für Fichte und Buche sind in Tabelle 1 und Tabelle 2 dokumentiert. Die Frontspalte gibt das jeweilige Bestandesalter am Anfang, die Kopfzeile das Bestandesalter am Ende der Betrachtungsperiode an. Die optimale Umtriebszeit ist dort, wo der jährliche Holzproduktionswert (ab dem Bestandesalter von 0 Jahren) maximal ist. Die Zahlen der Tabellen lassen sich hier beispielhaft für Fichte I. Ertragsklasse wie folgt interpretieren: 
Tabelle 7: Berechnung der jährlichen Holzproduktionswerte in $€ /$ ha in Abhängigkeit vom Bestandesalter und dem Bestandesalter am Ende der Betrachtungsperiode für Fichte, I. Ertragsklasse, mäßige Durchforstung (Durchmesserentwicklung adjustiert)

\begin{tabular}{|c|c|c|c|c|c|c|c|c|c|c|c|c|}
\hline & \multicolumn{12}{|c|}{ bis Alter...: jährlicher Holzproduktionswert in $€ /$ ha/Jahr (Fichte I. Ekl.) } \\
\hline von Alter... & 10 & 20 & 30 & 40 & 50 & 60 & 70 & 80 & 90 & 100 & 110 & 120 \\
\hline 0 (mit Kulturkosten) & -327 & -186 & -7 & 122 & 203 & 247 & 270 & 274 & 269 & 264 & 252 & 240 \\
\hline 0 (ohne Kulturkosten) & -23 & -23 & 109 & 216 & 283 & 318 & 334 & 334 & 326 & 318 & 304 & 290 \\
\hline 10 & & -23 & 192 & 322 & 393 & 423 & 432 & 422 & 407 & 392 & 372 & 353 \\
\hline 20 & & & 442 & 538 & 579 & 583 & 571 & 544 & 514 & 488 & 458 & 431 \\
\hline 30 & & & & 649 & 664 & 646 & 617 & 575 & 533 & 500 & 462 & 429 \\
\hline 40 & & & & & 681 & 643 & 603 & 548 & 498 & 459 & 415 & 378 \\
\hline 50 & & & & & & 599 & 554 & 489 & 432 & 391 & 343 & 303 \\
\hline 60 & & & & & & & 501 & 420 & 358 & 317 & 264 & 222 \\
\hline 70 & & & & & & & & 326 & 268 & 235 & 180 & 137 \\
\hline 80 & & & & & & & & & 201 & 178 & 115 & 69 \\
\hline 90 & & & & & & & & & & 151 & 60 & 10 \\
\hline 100 & & & & & & & & & & & -45 & -78 \\
\hline 110 & & & & & & & & & & & & -116 \\
\hline
\end{tabular}

Ausgehend von dem Bestandesalter von 0 Jahren treten bei kurzen Betrachtungsperioden jeweils negative jährliche Holzproduktionswerte auf, da den Kultur- und Bestandes-pflegekosten noch keine Erträge gegenüberstehen. Bei der Fichte (I. Ertragsklasse, siehe Tabelle 1) ergeben sich bei Berücksichtigung der Kulturkosten erstmals bei einer Produktionsdauer (Umtriebszeit) von 40 Jahren positive jährliche Holzproduktionswerte, bei einer Produktionsdauer von 80 Jahren ist mit 274 EUR/ha deren Maximum erreicht. Die höchsten laufenden jährlichen Holzproduktionswerte mit 681 EUR/ha zeigt die Fichte in der Altersphase von 40 auf 50 Jahre, hier findet eine besonders hohe Wertproduktion statt. Danach fallen die jährlichen Holzproduktionswerte wieder ab, in den hohen Altersphasen (ab Alter 100 Jahre) werden gar nur noch negative Holzproduktionswerte erzielt. Dies ist Folge der Tatsache, dass in den Altbeständen zunehmend Kapital gebunden wird, dessen Produktivität die Zinsforderung jedoch unterschreitet. Die ökonomische Hiebsreife ist dann bereits deutlich überschritten.

Bei der Buche (I. Ertragsklasse und unterstelltem Abfall der Wertziffer ab Alter 120; siehe Tabelle 2) zeigen sich, ausgehend vom Alter 0 und bei Einbeziehung der Kulturkosten jährliche Holzproduktionswerte von maximal -5 EUR/ha bei einer Produktionsdauer von 130 Jahren. Ohne Kulturkosten liegt der maximale durchschnittliche jährliche Holzproduktionswert bei 120 Jahren und beträgt 116 EUR/ha. Den maximalen laufenden jährlichen Holzproduktionswert erzielt die Buche in der Altersphase von 60 auf 70 Jahre mit 281 EUR/ha. Da davon auszugehen ist, dass die Kulturkosten für Buchenbestände in den meisten Fällen durch forstliche Förderung erstattet werden oder die Buche über Naturverjüngung begründet werden kann, werden in den folgenden Betrachtungen stets die Holzproduktionswerte für Buche ohne Kulturkosten betrachtet. 
Tabelle 8: Berechnung der jährlichen Holzproduktionswerte in $€ /$ ha in Abhängigkeit vom Bestandesalter und dem Bestandes-alter am Ende der Betrachtungsperiode für Buche, I. Ertragsklasse, mäßige Durchforstung (Durchmesserentwicklung adjustiert und Wertziffernabfall ab Alter 120 Jahre unterstellt)

\begin{tabular}{|c|c|c|c|c|c|c|c|c|c|c|c|c|c|c|c|}
\hline & \multicolumn{15}{|c|}{ bis Alter...: jährlicher Holzproduktionswert in $€ /$ ha/Jahr (Buche I. Ekl.) } \\
\hline von Alter... & 10 & 20 & 30 & 40 & 50 & 60 & 70 & 80 & 90 & 100 & 110 & 120 & 130 & 140 & 150 \\
\hline 0 (mit Kulturkosten) & -761 & -419 & -268 & -208 & -154 & -111 & -76 & -52 & -35 & -22 & -13 & -7 & -5 & -5 & -9 \\
\hline 0 (ohne Kulturkosten) & -23 & -23 & 15 & 19 & 40 & 62 & 81 & 94 & 104 & 110 & 114 & 116 & 114 & 111 & 105 \\
\hline 10 & & -23 & 39 & 38 & 63 & 88 & 109 & 123 & 133 & 139 & 142 & 144 & 141 & 136 & 129 \\
\hline 20 & & & 112 & 77 & 101 & 128 & 150 & 163 & 172 & 177 & 178 & 178 & 174 & 167 & 157 \\
\hline 30 & & & & 35 & 94 & 135 & 164 & 179 & 188 & 193 & 193 & 193 & 186 & 178 & 166 \\
\hline 40 & & & & & 162 & 197 & 221 & 231 & 234 & 235 & 232 & 229 & 219 & 208 & 192 \\
\hline 50 & & & & & & 238 & 258 & 261 & 260 & 258 & 252 & 246 & 232 & 218 & 198 \\
\hline 60 & & & & & & & 281 & 276 & 271 & 265 & 256 & 248 & 231 & 213 & 190 \\
\hline 70 & & & & & & & & 270 & 264 & 258 & 247 & 238 & 218 & 196 & 168 \\
\hline 80 & & & & & & & & & 257 & 251 & 237 & 227 & 202 & 176 & 143 \\
\hline 90 & & & & & & & & & & 243 & 224 & 213 & 182 & 151 & 112 \\
\hline 100 & & & & & & & & & & & 202 & 195 & 154 & 118 & 72 \\
\hline 110 & & & & & & & & & & & & 186 & 124 & 81 & 26 \\
\hline 120 & & & & & & & & & & & & & 53 & 16 & -46 \\
\hline 130 & & & & & & & & & & & & & & -28 & -107 \\
\hline 140 & & & & & & & & & & & & & & & -199 \\
\hline
\end{tabular}

\subsubsection{6. $\quad$ Anwendung auf Änderungen der forstlichen Bewirtschaftung}

Die im vorangegangenen Kapitel vorgestellten Grundlagen werden nachfolgend auf Änderungen der forstlichen Bewirtschaftung angewendet. Dabei können die Bewertungsfälle nochmals unterteilt werden in

1. Änderung bei der Bestandesbegründung und

2. Änderung der Bewirtschaftung eines vorhandenen Bestands.

\subsection{Vorübergehender Verzicht auf forstliche Produktion}

Der aus bewertungstechnischer Sicht einfachste Anwendungsfall ist der der aufgeschobenen Wiederbegründung eines endgenutzten Bestands zu anderen Zwecken (Freiflächenerhalt, natürliche Sukzession, etc.). Es wird unterstellt, dass eine solche Behandlung für den Eigentümer weder Einnahmen noch Ausgaben verursacht. Der entstehende Ertragsverlust entspricht dem Holzproduktionswert der ökonomischen Referenz (hier: Fichte) während der verhinderten oder aufgeschobenen Wiederaufforstung. Im Beispiel in Abbildung 31 ist dies ein 10-jähriger Zeitraum. Die Referenzbewirtschaftung der Fichte im 80-jährigen Umtrieb erzielt unter den gegebenen Bedingungen einen Holzproduktionswert von $274 € / h a / a$. Die alternative Bewirtschaftung (Offenhalten der Fläche) ist Null, so dass ein jährlicher Ertragsverlust von -274 €/ha/a resultiert. 


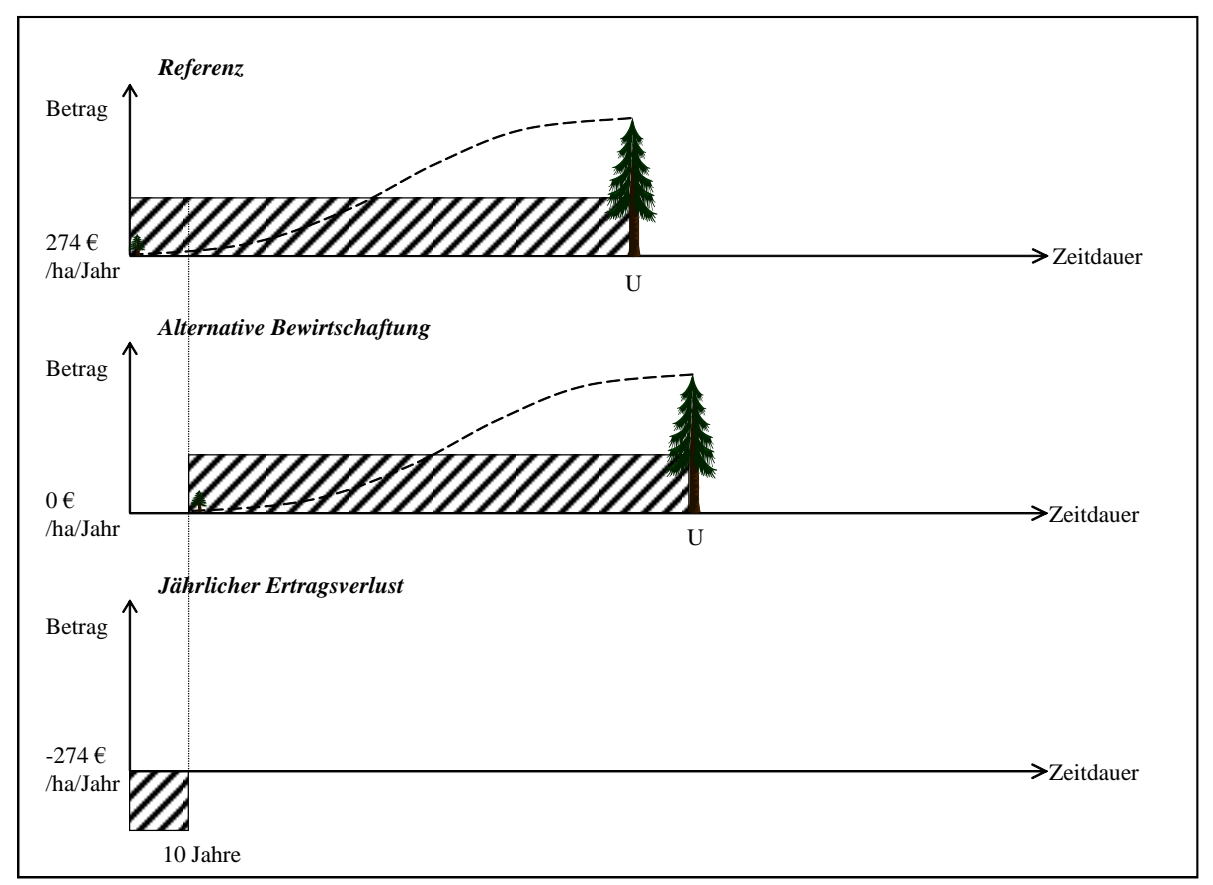

Abbildung 31: Aufschub/vorübergehender Verzicht auf forstliche Produktion

\subsubsection{2. Änderung der Baumartenwahl}

Eines der häufigsten Ziele der naturschutzfachlich ausgerichteten Waldbewirtschaftung ist der Umbau von nicht standortheimischen, aber produktiven Nadelbaumarten durch standortheimische, weniger produktive Laubbaumarten. Alternativ kann es auch der Verzicht auf die Einmischung von Nadelhölzern in vorhandene Laubholzbestände sein. Ein solcher Baumartenwechsel wirkt sich langfristig, bis zum Erreichen der Hiebsreife der gewünschten Baumart aus. Dieser langfriste Ertragsverlust kann am Beispiel Buche statt Fichte dargestellt werden (s. Abbildung 32).

Kann die naturschutzfachlich favorisierte Alternative erreicht werden, ohne dass Kulturkosten anfallen, z.B. durch Naturverjüngung oder durch vollständige Förderung der Kulturbegründungskosten, resultiert aus den Modell- und Datengrundlagen ein jährlicher Holzproduktionswert der Buche von $116 € / h a / a$. Die ökonomische Referenz Fichte erzielt wieder $274 €$ /ha/a. Daraus resultiert ein dauerhafter jährlicher Ertragsverlust von $-158 € /$ ha/a. 


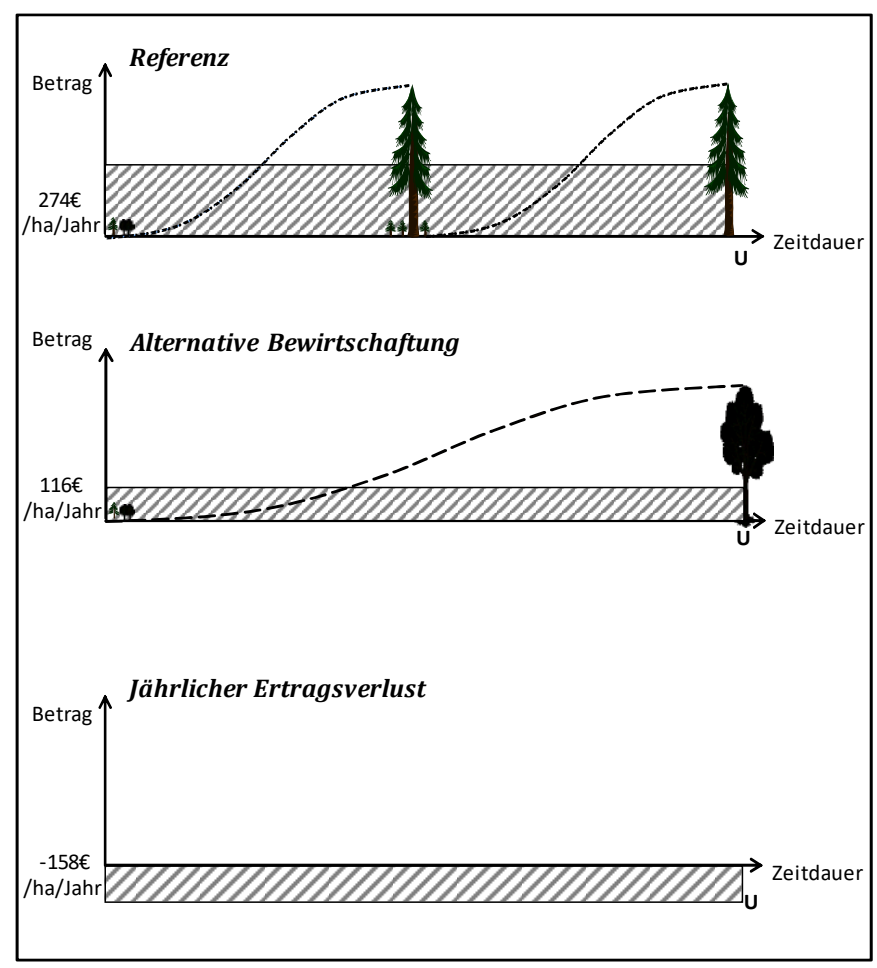

Abbildung 32: Änderung der Baumartenwahl (langer Betrachtungszeitraum)

Da in der Praxis ein laufender finanzieller Ausgleich des wirtschaftlichen Nachteils während des gesamten Wirkungszeitraumes des Baumartenwechsels (der gesamten Umtriebszeit) i.d.R. nicht in Frage kommt, werden im Folgenden die jährlichen, eine ganze Umtriebszeit umfassenden Ertragsverluste auf kürzere Ausgleichszeiträume umgerechnet. Dahinter steht die Vorstellung, dass Verträge über den Ausgleich von Mindererträgen aus dem Baumartenwechsel bspw. für eine Laufzeit von 30 Jahren im Zuge von Naturschutzmaßnahmen gegen Entgelt abgeschlossen werden können.

Als konzeptionelle Weiterentwicklung des bestehenden Bewertungskonzepts wurde eine Ausgleichstabelle entwickelt, die finanzmathematisch auf der Berechnung jährlich-endlicher Renten basiert (s. Formel 3).

Formel 3: Berechnung des Umrechnungsfaktors für Ausgleichszeitraum, Quelle: eigene Darstellung

$$
u f=\frac{(1+i)^{n}-1}{(1+i)^{n} \times i} \times \frac{(1+i)^{a} \times i}{(1+i)^{a}-1}
$$

$\begin{array}{lll}\text { uf } & = & \text { Umrechnungsfaktor } \\ \mathrm{n} & = & \text { Wirkungszeitraum } \\ \mathrm{i} & = & \text { Zinssatz } \\ \mathrm{a} & = & \text { Ausgleichszeitraum }\end{array}$


Für die Umrechnung der Ertragsnachteile der langen Wirkungszeiträume in kürzere Ausgleichszeiträume in Abhängigkeit des gewählten Zinssatzes (hier 1,5 \%) kann eine Faktortabelle erstellt werden, die eine unmittelbare Umrechnung der langfristigen Holzproduktionswerte ermöglicht (s. Tabelle 9).

Tabelle 9: Umrechnungsfaktoren von langfristigen Wirkungszeiträumen in kurzfristige Ausgleichszeiträume (berechnet auf der Basis eines Kalkulationszinses von 1,5\%)

\begin{tabular}{|c|c|c|c|c|c|c|c|c|c|c|c|c|c|c|}
\hline \multirow{2}{*}{\multicolumn{2}{|c|}{$\begin{array}{r}\text { Zins: } \begin{array}{r}1,5 \% \\
\text { Jahre }\end{array}\end{array}$}} & \multicolumn{13}{|c|}{ Wirkungszeitraum } \\
\hline & & 60 & 70 & 80 & 90 & 100 & 110 & 120 & 130 & 140 & 150 & 160 & 170 & 180 \\
\hline \multirow{8}{*}{ 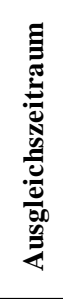 } & 5 & 8,23 & 9,02 & 9,70 & 10,29 & 10,79 & 11,23 & 11,60 & 11,93 & 12,21 & 12,45 & 12,65 & 12,83 & 12,98 \\
\hline & 10 & 4,27 & 4,68 & 5,03 & 5,34 & 5,60 & 5,82 & 6,02 & 6,19 & 6,33 & 6,45 & 6,56 & 6,65 & 6,73 \\
\hline & 15 & 2,95 & 3,23 & 3,48 & 3,69 & 3,87 & 4,02 & 4,16 & 4,28 & 4,37 & 4,46 & 4,53 & 4,60 & 4,65 \\
\hline & 20 & 2,29 & 2,51 & 2,70 & 2,87 & 3,01 & 3,13 & 3,23 & 3,32 & 3,40 & 3,47 & 3,52 & 3,57 & 3,62 \\
\hline & 25 & 1,90 & 2,08 & 2,24 & 2,38 & 2,49 & 2,59 & 2,68 & 2,75 & 2,82 & 2,87 & 2,92 & 2,96 & 3,00 \\
\hline & 30 & 1,64 & 1,80 & 1,93 & 2,05 & 2,15 & 2,24 & 2,31 & 2,38 & 2,43 & 2,48 & 2,52 & 2,56 & 2,59 \\
\hline & 35 & 1,45 & 1,59 & 1,71 & 1,82 & 1,91 & 1,98 & 2,05 & 2,11 & 2,16 & 2,20 & 2,23 & 2,27 & 2,29 \\
\hline & 40 & 1,32 & 1,44 & 1,55 & 1,64 & 1,73 & 1,80 & 1,86 & 1,91 & 1,95 & 1,99 & 2,02 & 2,05 & 2,08 \\
\hline
\end{tabular}

Auf ein Beispiel (Abbildung 33) bezogen führt das zu folgendem Ergebnis:

Die Referenzbewirtschaftung der Fichte liegt wieder bei $274 € /$ ha/a, die ökologische Alternative bei den bereits bekannten $116 € /$ ha/a. Bezogen auf einen 30 -jährigen Ausgleichszeitraum bei einem Wirkungszeitraum von 120 Jahren (Umtriebszeit Buche) kann mit dem Faktor von 2,31 (s. Tabelle 9) die jährliche Differenz auf einen Ausgleichsbetrag von -365 $€ /$ ha/a umgerechnet werden, wobei hier zu betonen ist, dass dieser Betrag als jährlicher Zahlungsbetrag nur für einen Zeitraum von 30 Jahren anzusehen ist. Diese, bspw. vertraglich zu vereinbarende, befristete Zahlung in der Jugendphase von Laubholzbeständen, dürfte auch mit den Liquiditätsbedürfnissen der Forstbetriebe korrespondieren, denn in dieser Phase sind die Zahlungsdifferenzen relativ hoch. 


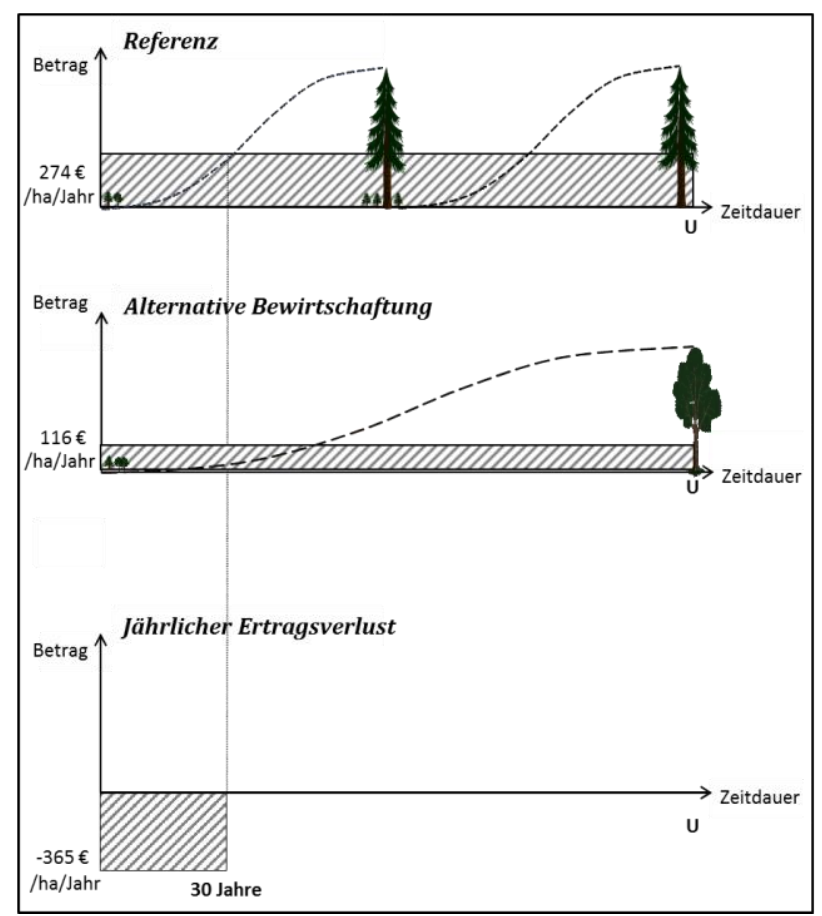

Abbildung 33: Änderung der Baumartenwahl (Verrechnung auf 30-jährigen Ausgleichszeitraum)

\subsection{Vorzeitige Ernte eines Bestands}

Als erstes Beispiel der Änderung der Bewirtschaftung eines vorhandenen Bestands soll hier dessen vorzeitige Ernte betrachtet werden. Diese kann bspw. erfolgen, wenn ein Biotop freigestellt werden soll oder eine andere Baumart etabliert wird.

Mit der vorzeitigen Ernte geht das noch nicht realisierte zukünftige Ertragspotential verloren. In der Waldbewertung wird in einem solchen Fall häufig die sog. Hiebsunreife als Differenz zwischen aktuellem Abtriebswert und Erwartungswert ermittelt. Das Bewertungskonzept bietet in diesem Fall alternativ die Herleitung des Differenzbetrags über die tabellarisierten jährlichen Holzproduktionswerte.

Im Beispiel (Abbildung 34) wird ein nicht hiebsreifer 40-jähriger Fichtenbestand zugunsten einer Freifläche flächig entfernt, so dass dem Forstbetrieb der von diesem Bestand noch zu erwartende jährliche Holzproduktionswert entgeht.

Als Referenz ist zunächst der Holzproduktionswert der Periode zwischen dem aktuellen Alter (40 Jahre) und dem Endnutzungszeitpunkt (Alter 80 Jahre) anzusetzen. Ein Fichtenbestand erbringt in dieser hochproduktiven Phase einen Holzproduktionswert von $548 € /$ ha/a (s. Tabelle 7). Die alternative Nutzung als Freifläche ist mit einem Ertrag von Null anzusetzen. Die entstehende jährliche Differenz der beiden Alternativen während des erwähnten Zeitraumes liegt bei $-548 € / h a / a$. 


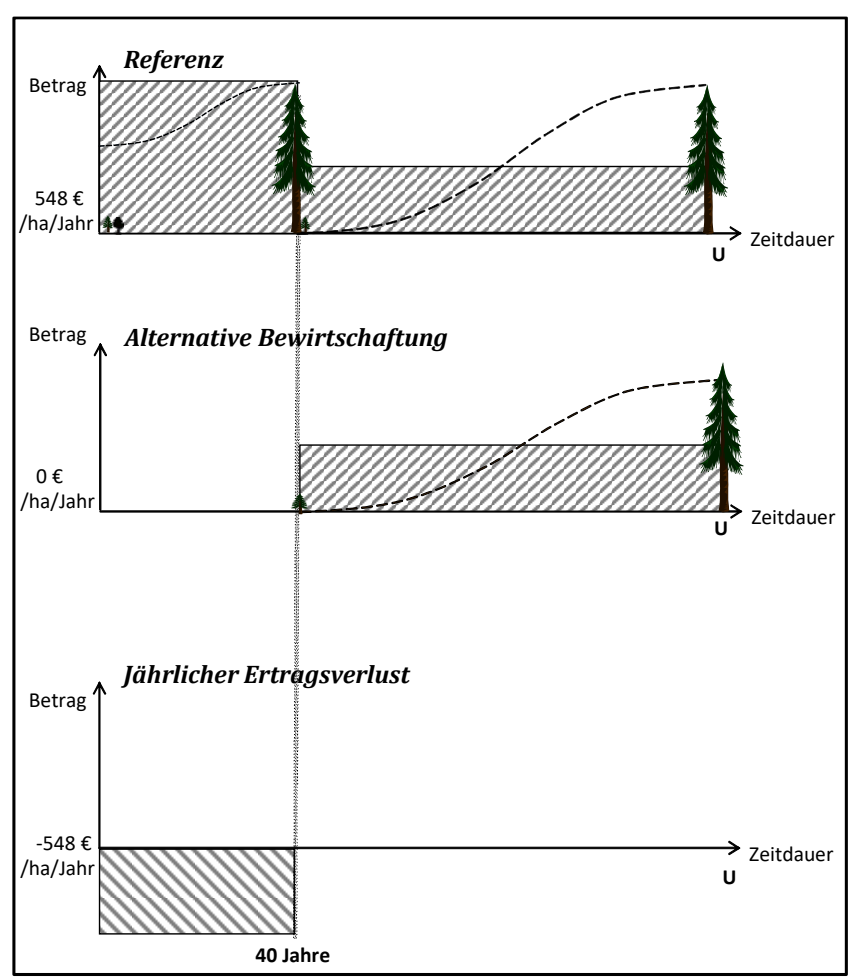

Abbildung 34: Vorzeitige Ernte eines Bestands

\subsection{Erhalt eines hiebsreifen Bestands}

Der gegenteilige, aber bewertungstechnisch ähnliche Fall liegt vor, wenn ein Bestand über seine forstlich optimale Umtriebszeit hinaus erhalten werden soll. Planmäßig erfolgte nach der Nutzung des hiebsreifen Bestands unmittelbar die Begründung des forstlich optimalen Nachfolgebestands. Der (vertraglich vereinbarte oder vorgeschriebene) Erhalt des aktuellen Bestands verhindert dies und gleichfalls den jährlichen Holzproduktionswert des Nachfolgebestands. Gegenzurechnen ist allerdings der Ertrag, den der erhaltene Bestand noch realisieren kann. Sollte dieser allerdings aufgrund des hohen Alters durch Entwertung negativ sein, ist er zusätzlich auszugleichen.

Ein solches Beispiel ist auf Basis der Datengrundlagen für die Buche nur dann plausibel darstellbar, wenn unterstellt wird, dass die Holzqualität (Wertziffer) über das Alter durch die Bildung von Rot- oder Spritzkern absinkt. Im vorliegenden Modell wurde für die Buche die Wertziffer (WZ) ab dem Alter 120 Jahre von WZ 3 bis zum Alter 150 auf die WZ 5 abgesenkt.

Für die ökonomische Referenz ergibt sich ein Holzproduktionswert von $274 € /$ ha/a. Der Erhalt des Bestands als ökologische Alternative verursacht durch die Entwertung des Holzes einen negativen Holzproduktionswert von $-46 € /$ ha/a für die nächsten 30 Jahre. Daraus resultiert eine Differenz von $-320 € /$ ha/a, die für den Erhalt des Buchenbestands auszugleichen wäre. 
Alternativ böte sich, wie in den Richtlinien für die Begründung von Nutzungsverhältnissen an Waldflächen für Zwecke der Verteidigung (Bundesminister der Justiz 1986, S. 7) ein anderes Vorgehen an. Insbesondere wenn die Entwertungsrisiken nicht kalkulierbar sind, kann der aktuelle Bestand zum jetzigen Abtriebswert, inkl. Übertragung der Verfügungsrechte, käuflich erworben werden. Zum Ende der Vertragslaufzeit wird der Bestand dann entweder vom Käufer verwertet oder ihn zum dann gültigen Abtriebswert dem Verkäufer wieder überlassen. Für den Vertragszeitraum ist durch den Käufer zusätzlich der Nutzenentgang in Form der Bodenbruttorente zu erstatten.

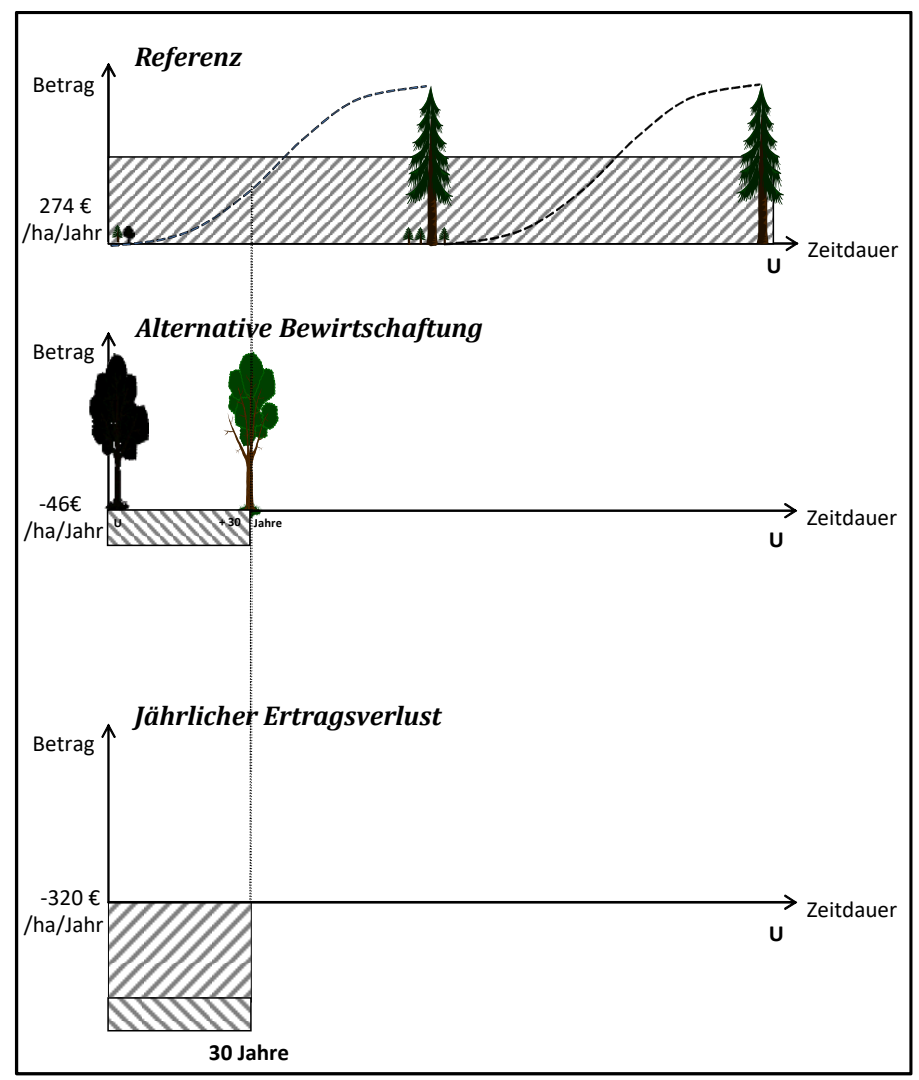

Abbildung 35: Erhalt eines hiebsreifen Bestands

\subsection{Erhalt von Alt- und Totholz (Zerfallsphase)}

In aktuellen naturschutzpolitischen Debatten spielt die Schaffung von ökologisch wertvollen Altholzinseln, die als Habitate oder Totholzquellen genutzt werden können, eine große Rolle. Hierzu wird bei Beständen, die ihre Umtriebszeit erreicht haben auf die Endnutzung verzichtet und sie werden der weiteren natürlichen Alterung bis hin zur Absterbe- und Zerfallsphase überlassen. Aus betriebswirtschaftlicher Bewertungssicht werden somit hiebsreife Bestände durch Zerfall sukzessive vernichtet. 
Die Berücksichtigung des Erhalts von Alt- und Totholz bis zur Zerfallsphase bedurfte der konzeptionellen Weiterentwicklung, da sie im ersten Bewertungskonzept (s. Möhring und Rüping 2006) noch nicht explizit berücksichtigt wurde. Als Neuerung wird der Vorschlag gemacht, diese Phase ökonomische mittels einer „(Teilwert-) Abschreibung“ des aktuellen Abtriebswertes über einen festzulegenden Zeitraum darzustellen. Zusätzlich zu der sukzessiven Entwertung des Abtriebswertes muss auch in diesem Fall zur Bewertung der entgehenden Flächenerträge wieder auf die Referenzbestockung zurückgegriffen werden, da die Fläche durch die vorhandenen Alt- und Habitatbäume einer anderweitigen Nutzung entzogen ist.

Die jährlichen „Abschreibungsbeträge“ lassen sich ebenfalls tabellarisiert darstellen. Hierzu folgendes Beispiel:

a) Referenz: Jährlicher Holzproduktionswert aus dem forstlich optimalen Nachfolgebestand; Fichte mit einer Umtriebszeit von 80 Jahren; $274 € /$ ha und Jahr.

b) Alternative Bewirtschaftung: Jährlicher Wertverlust durch Erhalt des hiebsreifen Buchenaltholzbestandes (Abtriebswert $16.333 € /$ ha) bis zum vollständigen Zerfall nach 30 Jahren; $-686 € /$ ha und Jahr.

c) Jährlicher Ertragsverlust während der nächsten 30 Jahre: Resultierend aus dem Entgang des Folgebestandes sowie der Wertverluste des abzuschreibenden Altbestandes: $-960 € /$ ha und Jahr.

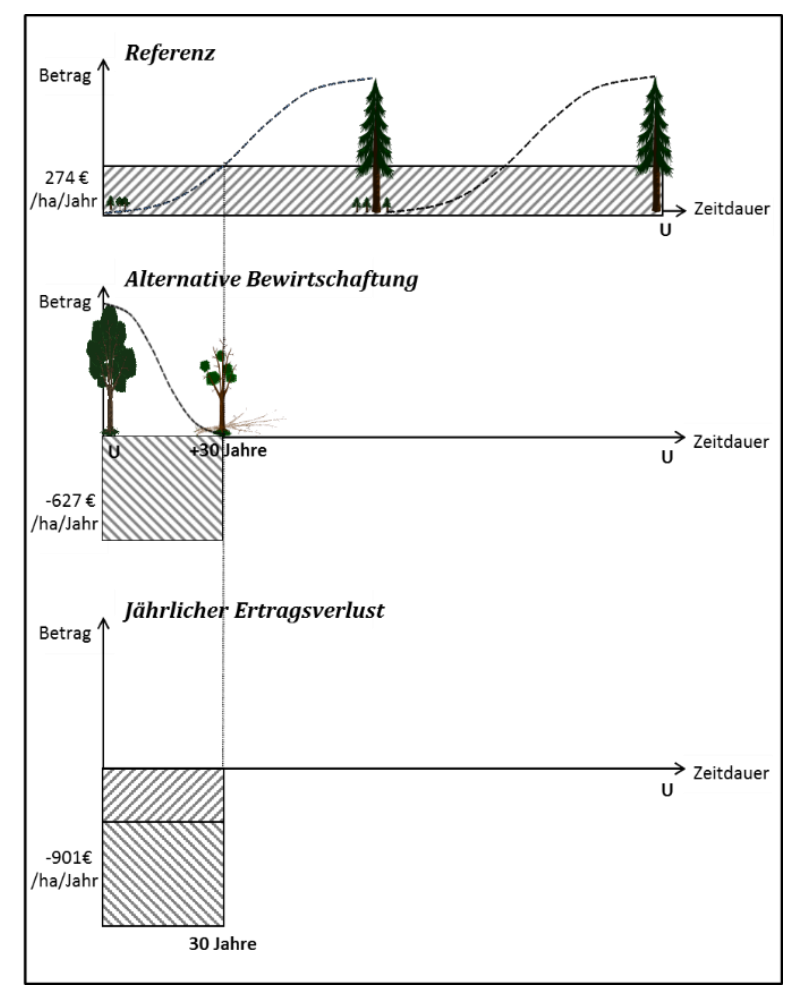

Abbildung 36: Erhalt von Habitatbäumen 
Bei der Ermittlung der jährlichen Ertragsverluste kann auf Tabelle 10 zurückgegriffen werden, die in Abhängigkeit von der Dauer und dem abzuschreibenden Wertanteil den jährlichen Betrag in das Verhältnis zum aktuellen Abtriebswert setzt. Dies erfolgt durch die Berechnung des Quotienten von Annuität und diskontierter Wertminderung, unter Berücksichtigung des Zeitraums und des angenommenen Restwerts.

Formel 4: Ermittlung des jährlichen Ertragsverlusts in \% des Abtriebswertes unter Berücksichtigung des Zinses, der Laufzeit und der unterstellten Wertminderung, Quelle: eigene Darstellung

$$
\text { jährlicher Ertragsverlust in } \%=\left(1-\frac{R_{n}}{(1+i)^{n}}\right) \times \frac{(1+i)^{n} \times i}{(1+i)^{n}-1}
$$

$$
\begin{array}{lll}
\mathrm{R}_{\mathrm{n}} & = & \text { Restwert zum Zeitpunkt } \mathrm{n} \text { in Prozent des aktuellen Abtriebswertes } \\
\mathrm{n} & = & \text { Wirkungszeitraum } \\
\mathrm{i} & = & \text { Zinsatz in } \%
\end{array}
$$

So führt bspw. ein Abtriebswert von $16.333 € /$ ha, der über einen Zeitraum von 30 Jahren vollständig wertgemindert (abgeschrieben) wird, zu einem jährlichen Ertragsverlust von $-686 € / \mathrm{ha} / \mathrm{Jahr}(16.333 € / \mathrm{ha} * 4,2 \%=686 € / \mathrm{ha} / \mathrm{Jahr})$. Wird der gleiche Abtriebswert in diesem Zeitraum lediglich zu 50 \% gemindert, errechnet sich ein jährlicher Ertragsverlust von $457 € /$ ha $(16.333 € /$ ha * 2,8 \% = $457 € /$ ha/Jahr). Der Zusammenhang ist aufgrund des Zinsein-

\begin{tabular}{|c|c|c|c|c|c|c|c|c|c|c|c|c|}
\hline & & \multicolumn{11}{|c|}{ Restwert } \\
\hline & & $100 \%$ & $90 \%$ & $80 \%$ & $70 \%$ & $60 \%$ & $50 \%$ & $40 \%$ & $30 \%$ & $20 \%$ & $10 \%$ & $0 \%$ \\
\hline \multirow{8}{*}{ 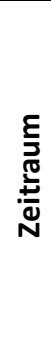 } & 5 & $1,5 \%$ & $3,4 \%$ & $5,4 \%$ & $7,3 \%$ & $9,3 \%$ & $11,2 \%$ & $13,1 \%$ & $15,1 \%$ & $17,0 \%$ & $19,0 \%$ & $20,9 \%$ \\
\hline & 10 & $1,5 \%$ & $2,4 \%$ & $3,4 \%$ & $4,3 \%$ & $5,2 \%$ & $6,2 \%$ & $7,1 \%$ & $8,0 \%$ & $9,0 \%$ & $9,9 \%$ & $10,8 \%$ \\
\hline & 15 & $1,5 \%$ & $2,1 \%$ & $2,7 \%$ & $3,3 \%$ & $3,9 \%$ & $4,5 \%$ & $5,1 \%$ & $5,7 \%$ & $6,3 \%$ & $6,9 \%$ & $7,5 \%$ \\
\hline & 20 & $1,5 \%$ & $1,9 \%$ & $2,4 \%$ & $2,8 \%$ & $3,2 \%$ & $3,7 \%$ & $4,1 \%$ & $4,5 \%$ & $5,0 \%$ & $5,4 \%$ & $5,8 \%$ \\
\hline & 25 & $1,5 \%$ & $1,8 \%$ & $2,2 \%$ & $2,5 \%$ & $2,8 \%$ & $3,2 \%$ & $3,5 \%$ & $3,8 \%$ & $4,2 \%$ & $4,5 \%$ & $4,8 \%$ \\
\hline & 30 & $1,5 \%$ & $1,8 \%$ & $2,0 \%$ & $2,3 \%$ & $2,6 \%$ & $2,8 \%$ & $3,1 \%$ & $3,4 \%$ & $3,6 \%$ & $3,9 \%$ & $4,2 \%$ \\
\hline & 35 & $1,5 \%$ & $1,7 \%$ & $1,9 \%$ & $2,2 \%$ & $2,4 \%$ & $2,6 \%$ & $2,8 \%$ & $3,0 \%$ & $3,3 \%$ & $3,5 \%$ & $3,7 \%$ \\
\hline & 40 & $1,5 \%$ & $1,7 \%$ & $1,9 \%$ & $2,1 \%$ & $2,2 \%$ & $2,4 \%$ & $2,6 \%$ & $2,8 \%$ & $3,0 \%$ & $3,2 \%$ & $3,3 \%$ \\
\hline
\end{tabular}
flusses nicht linear.

Tabelle 10: Jährliche Ertragsverluste in Prozent des Abtriebswertes, in Abhängigkeit von der Höhe des Wertverlustes (Restwert \%) und des Wirkungszeitraums (bei unterstelltem Zins von 1,5\%)

\subsubsection{7. Änderungen der Datengrundlagen zwischen 2006 und 2015}

Das hier vorgestellt Bewertungskonzept ist eine gegenüber 2006 aktualisierte und erweiterte Fassung. Wie bereits ausgeführt haben deutliche Änderungen am Holzmarkt sowie die in der Praxis stattfindenden Bewertungsfälle diese Erneuerung notwendig gemacht. Nachfolgend sollen die Veränderungen der Holzproduktionswerte vergleichend dargestellt werden. 
Abbildung 37 stellt die jeweils drei maximalen Holzproduktionswerte in den ersten drei Ertragsklassen der fünf Hauptbaumarten ohne Berücksichtigung der Kulturkosten aus den Jahren 2006 und 2015 gegenüber. Die gestrichelt eingezeichnete Winkelhalbierende steht dabei für keine Veränderung von 2006 zu 2015. Punkte oberhalb dieser Linie werden durch einen Anstieg der jeweiligen Holzproduktionswerte der Baumart charakterisiert. Punkte unterhalb durch ein Absinken. Zu erkennen ist, dass die Buche im Vergleich der Baumarten die geringsten Holzproduktionswerte erwarten lässt und sich im Vergleich zu 2006 überwiegend verschlechtert hat. Alle anderen Baumarten haben sich aufgrund der gestiegenen Holzerlöse verbessert. Besonders für die Fichte und die Douglasie hat ein deutlicher Anstieg der Holzproduktionswerte stattgefunden.

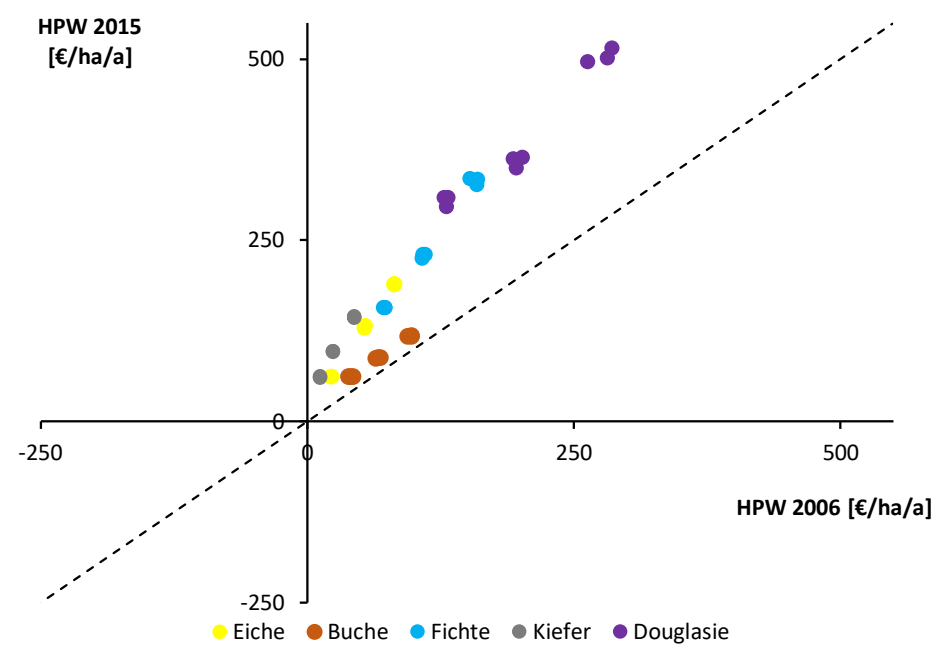

Abbildung 37: Veränderung der Holzproduktionswerte der fünf Hauptbaumarten von 2006 zu 2015 ohne Berücksichtigung der Kulturkosten

Abbildung 38 ist inhaltlich identisch zu Abbildung 37, jedoch wurden die Holzproduktionswerte ohne Berücksichtigung von Kulturkosten berechnet. Auffallend ist, dass sich alle Baumarten im Jahr 2015 zu 2006 verbessert haben. Die geringste Verbesserung weist hier die Buche auf. Insoweit wird erkennbar, dass insbesondere bei der Buche die gestiegenen Bestandesbegründungskosten einen großen Einfluss auf die berechneten Werte haben 


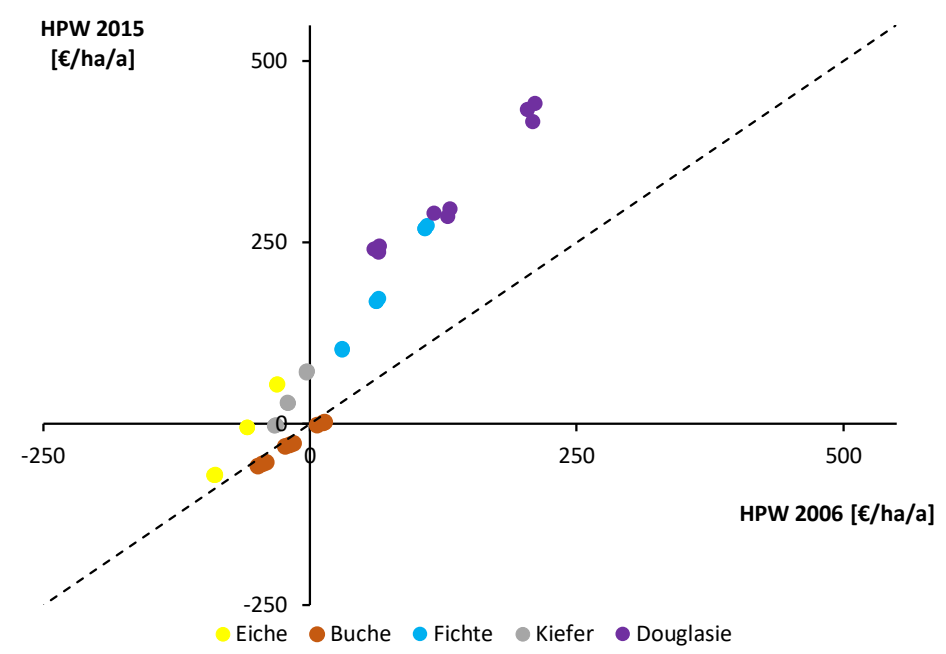

Abbildung 38: Veränderung der Holzproduktionswerte der fünf Hauptbaumarten von 2006 zu 2015 unter Berücksichtigung der Kulturkosten

Neben der Aktualisierung der Datenbasis hat mit der Alt- und Habitatbaumbewertung auch ein wichtiger neuer Bewertungsansatz Eingang in das Konzept gefunden. Zusätzlich wurde das ursprüngliche Konzept von 2006 um die Umrechnungstabellen für kürzere Zeiträume sowie die Wertminderungsprozente bei Alt- und Habitatbäumen ergänzt.

\subsubsection{Vergleich von Bewertungstools für forstliche Nutzungsbeschränkungen}

Auf Grundlage des in Kapitel 4.3.1 erläuterten Bewertungskonzepts existieren in der Praxis mehrere Kalkulationsinstrumente, die regelmäßig für die beschriebenen Bewertungsfälle herangezogen werden. Nachfolgend soll synoptisch dargestellt werden, welche unterschiedlichen Eingangsgrößen in den Kalkulationsprogrammen Berücksichtigung finden und welchen Einfluss dies auf die berechneten Ergebnisse hat.

Für die Analyse lag das zum Bewertungskonzept originär erstellte Bewertungstool der Abteilung Forstökonomie der Georg-August-Universität Göttingen vor. Weiterhin wurden die Bewertungstools vom Landesbetrieb HessenForst (HF) und der Anstalt öffentlichen Rechts Niedersächsische Landesforsten (NLF) untersucht. Diese Tools werden von den Institutionen einerseits intern für Bewertungsfälle herangezogen, zeitgleich aber auch Dritten entgeltlich zur Verfügung gestellt. ${ }^{37}$ Das Tool der Abteilung Forstökonomie, das vom Autor überarbeitet wurde, wird vorrangig zu Lehr- und Forschungszwecken verwendet. Alle drei Tools basieren auf der Tabellenkalkulationssoftware Microsoft Excel und besitzen somit eine breite Anwendergruppe. 37 https://www.hessen-forst.de/marktplatz// \& https://www.landesforsten.de/bewirtschaften/unsere-
dienstleistungen/waldbewertung/ 


\subsubsection{Grundsätze der Bewertungstools}

1. Basierend auf dem Bewertungskonzept für forstliche Nutzungsbeschränkungen verfolgen alle Tools das Ziel, Veränderungen der forstlichen Produktion durch ökonomische Kennziffern zu bewerten. Dabei erfolgt stets die Berechnung eines Holzproduktionswertes (HPW) für spezifische Bewertungsobjekte.

2. Wie in Kapitel 4.3.1 erläutert wird der Holzproduktionswert, zu interpretieren als jährlicher Deckungsbeitrag der waldbaulichen Holzproduktion inkl. der Kapitalkosten für entscheidungsrelevante Zahlungen vor Abzug der jährlichen Fixkosten, in der Form: „Kapitalwert x Wiedergewinnungsfaktor“ in allen Waldbewertungstools gleich ermittelt.

3. Anpassungen an den jeweiligen Standort bzw. Besonderheiten des Bewertungsobjektes sind möglich und nötig.

\subsubsection{Inputgrößen}

Die Bewertungstools von HessenForst und den Niedersächsischen Landesforsten sind für regionale Besonderheiten angepasst worden. Insofern sind Unterschiede in den Eingangsdaten zu erwarten.

In Tabelle 11 sind die relevanten Eingangsgrößen vergleichend nebeneinander aufgeführt. Aufgrund der spezifischen zugrundeliegenden Waldbewertungsrichtlinien der Bundesländer variiert eine Vielzahl von Basisdaten. Beispielsweise verwendet Niedersachsen zur Bonitierung von Beständen das System der Leistungsklassen, wohingegen die Tools der Universität Göttingen und Hessens, basierend auf den unterschiedlichen Ertragstafeln, in Ertragsklassen bonitieren. 
Tabelle 11: Eingangsdaten der verglichenen Kalkulationstools für forstliche Nutzungsbeschränkungen. Zur besseren Lesbarkeit befindet sich diese Tabelle erneut im Anhang 9.1.5.

\begin{tabular}{|c|c|c|c|}
\hline & Hessen & Niedersachsen & Uni Göttingen (WBR NRW) \\
\hline Eingangsdaten & $\begin{array}{l}8 \text { Baumarten, Zuteilung von Nebenbaumarten zu } \\
\text { Hauptbaumarten-ET festgelegt }\end{array}$ & $\begin{array}{l}14 \text { Baumarten, Zuteilung von Nebenbaumarten zu } \\
\text { Hauptbaumart-ET festgelegt }\end{array}$ & Hauptbaumarten sind in Dateien hinterlegt \\
\hline Umtriebszeit & $\begin{array}{l}\text { Maximalwertempfehlungen hinterlegt (Bu max. } \\
240 \text { Jahre), Kalkulationshilfe gibt } 140 \text { Jahre an }\end{array}$ & $\begin{array}{l}\text { festgelegte Umtriebszeiten baumartspezifisch (Bu } \\
140 \text { Jahre) }\end{array}$ & frei wählbar \\
\hline Ertragsklasse/Leistungsklasse & Ertragsklassen hinterlegt ( $\mathrm{Bu} 0,0$ bis 4,0 ) & $\begin{array}{l}\text { Leistungsklasse } 1 \text { bis 14; Umrechnungsfaktoren } \\
\text { von Ertragsklassen in Leistungsklassen }\end{array}$ & Ertragsklassen hinterlegt (Buche 1 bis 3 ) \\
\hline Wertklasse & $\begin{array}{l}1 \text { (überdurchschnittlich) bis } 3 \\
\text { (unterdurchschnittlich), durch Verzögerung der EN } \\
\text { ist Wertabfall modellierbar }\end{array}$ & 1 bis 3 & $\begin{array}{l}\text { Wertziffer: } 3 \text { gute Qualität; } 1 \& 2 \text { mit hohen } \\
\text { Wertholzanteilen, Wertklassenänderung im Laufe } \\
\text { des Alters modellierbar }\end{array}$ \\
\hline Erntekosten & $\begin{array}{l}1 \text { (normal) oder } 2 \text { (schwierig), Erntekosten für dg } \\
\text { berechnet }\end{array}$ & 1 (einfach), 2 (normal), 3 (schwierig) & 1 (günstig) bis 6 (teuer) \\
\hline Kulturkosten & $\begin{array}{l}\text { individuell eintragbar, Empfehlungen in } \\
\text { Kalkulationshilfe einfache Verhältnisse, mittlere } \\
\text { Verhältnisse, schwierige Verhältnisse }\end{array}$ & $\begin{array}{l}1 \text { (geringwertige Bestände) bis } 6 \text { (Bestände aus } \\
\text { Kulturen) }\end{array}$ & $\begin{array}{l}\text { frei wählbar, zusammen mit } \\
\text { Bestandespflegekosten als Waldbaukosten, } \\
\text { Empfehlungen aus WBR NRW }\end{array}$ \\
\hline Sortentafel & in WBR oder Bewertungstool nicht vorhanden & $\begin{array}{l}\text { in Bewertungstool hinterlegt, wird für } \\
\text { Erlöskalkulation genutzt, um Güte- \& } \\
\text { Stärkeklassenanteile für jeweiliges Szenario zu } \\
\text { berechnen, keine Durchschnittswerte; } \\
\text { Massenverlust-Prozent }\end{array}$ & $\begin{array}{l}\text { in WBR enthalten, im Bewertungstool finden sich } \\
\text { Durchschnittswerte nach BHD, die anhand der } \\
\text { Sortentafel ermittelt wurden }\end{array}$ \\
\hline Zins & $1,5 \%$ als Standard, frei wählbar & & \\
\hline Bestandespflegekosten & $\begin{array}{l}\text { baumartenspezifisch unterteilt in Naturverjüngung } \\
\text { od. Pflanzung }\end{array}$ & individuell eintragbar im Alter 0 bis 30 & $\begin{array}{l}\text { individuell eintragbar im Alter } 0 \text { bis } 30 \text {, zusammen } \\
\text { mit Kulturkosten in Zeile Waldbaukosten }\end{array}$ \\
\hline Umrechnung Vfm in Efm & $\begin{array}{l}\text { aus Tabelle spezifisch für Baumart und dg } \\
\text { berechnet }\end{array}$ & Vfm in Efm: 0,8 & Vfm in Efm: 0,8 \\
\hline Holzerlöse & Buche $D g=60$ Erlös in $€ / E f m$ (Brutto): 73,33 & $\begin{array}{l}\text { Buche, Stärkeklasse } 4: 88 € / \text { fm }(70,40 € / E f m) \text {--> } \\
\text { Standardsorte; Erhöhungs-/ Reduktionsfaktoren für } \\
\text { jede Baumart nach Güte- } \& \text { Stärkeklasse; Erlöse } \\
\text { werden einzeln in Datenblatt berechnet aus } \\
\text { Stammholzerlösen, Nichtstammholzerlösen, } \\
\text { Reduktionsfaktoren und Erntekosten }\end{array}$ & $\begin{array}{l}\text { ausgedrückt durch Wertziffern, die von Wertziffer } \\
1 \text { bis } 6 \text { für BHD von } 10 \text { bis } 70 \mathrm{~cm} \text {, Erlöse Wertziffer } \\
1 \text { u. } 2 \text { sind erst ab BHD }=55 \text { höher als Wertziffer } 3 \text {, } \\
\text { da kein Wertholz in geringen Dimensionen }\end{array}$ \\
\hline Untergrenze BHD & nicht angegeben & $10 \mathrm{~cm}$ & frei wählbar \\
\hline $\begin{array}{l}\text { Ertragstafel (beispielhaft für } \\
\text { Buche) }\end{array}$ & Wiedemann, 1931, mäßige Durchforstung & Schober, 1967, mäßige Durchforstung & Schober, 1967, mäßige Durchforstung \\
\hline Ertragstafelkalibrierung & $\begin{array}{l}\text { Iz und dg kalibriert (gemäß hessischen ET- } \\
\text { Kalibrierungsfunktion), Beispiel Buche100, Ekl. 1, } \\
\operatorname{dg}(\text { verbl.): } \operatorname{dg}(\mathrm{ET})=32, \operatorname{dg}(\text { kal) }=38,8\end{array}$ & $\begin{array}{l}\text { dg nach Wollborn und Böckmann kalibriert, } \\
\text { Beispiel Buche100, Lkl. } 9 \text { (aus Tabelle Ekl } 1 \text { zu Lkl. = } \\
8,6) d g(E T)=35, d g(k a l)=44,2\end{array}$ & $\begin{array}{l}\text { BHD kalibriert nach Wollborn und Böckmann; } \\
\text { Buche 100, Ekl.1, BHD (verbl.) : BHD(ET)=33,6, } \\
\operatorname{dg}(\text { kal })=43\end{array}$ \\
\hline
\end{tabular}

Aufgrund der freien Wählbarkeit einer Vielzahl von Parametern lassen sie die Grunddaten jedoch auch verhältnismäßig einfach an einen besonderen Bewertungsfall anpassen. Besonderen Einfluss auf die Kalkulationsergebnisse haben die Holzerntekosten und -erlöse. Im Vergleich bspw. der Baumart Buche für eine mittlere Wertklasse, bzw. -ziffer zeigt sich, dass die hier verwandten nordrhein-westfälischen Grundlagen das höchste Niveau aufweisen, während Hessen und Niedersachsen absteigend folgen (s. Abbildung 39).

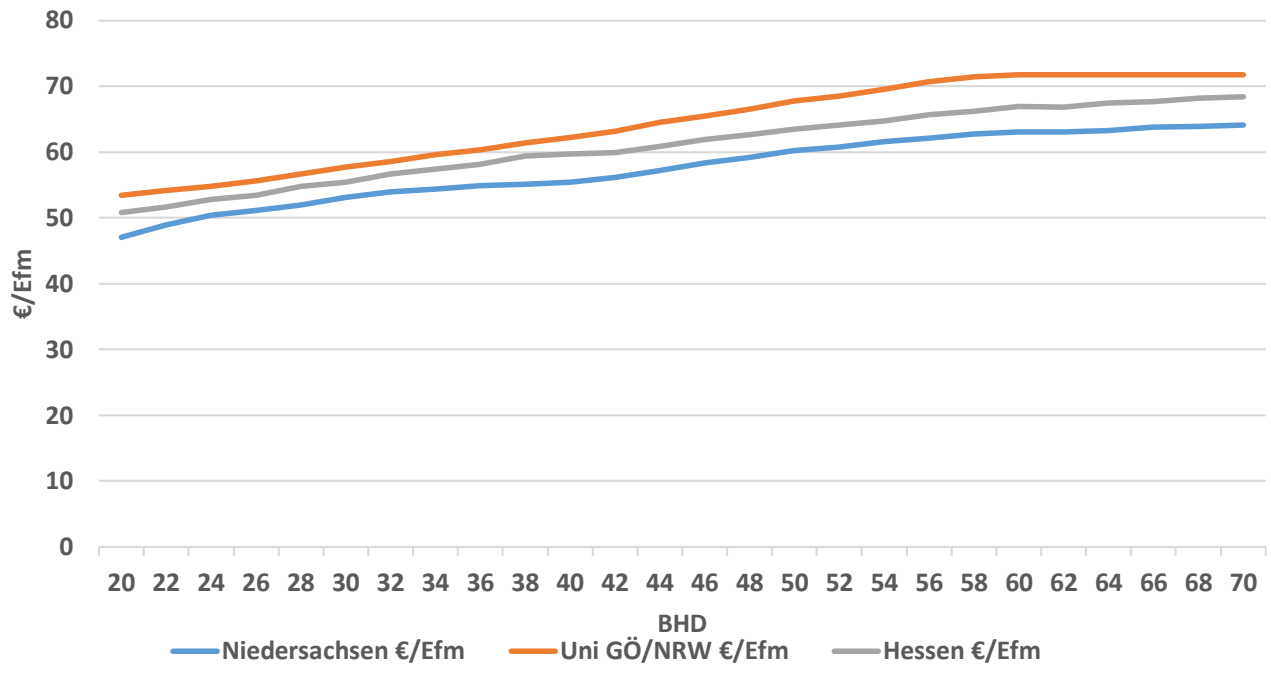

Abbildung 39: Vergleich Holzerlös Buche Wertklasse 2, bzw. Wertziffer 4 in €/Efm in Abhängigkeit des BHD für die drei Kalkulationstools 
Der Verlauf der Erlöskurven ist dabei in allen drei Fällen sehr ähnlich. Da sich sowohl die niedersächsischen als auch die nordrhein-westfälischen Werte aus dem gleitenden Durchschnitt der vergangenen Jahre errechnen, ist bei ähnlicher Situation an den Holzmärkten ein ähnliches Niveau erwartbar.

\subsubsection{Vergleich zentrale Bewertungsfälle}

Die Bewertungstools basieren auf dem gleichen methodischen Konzept mit den im vorangegangenen Kapitel dargestellten unterschiedlichen Eingangsdaten. Der Einfluss dieser Eingangsparameter äußert sich in den berechneten Ergebnissen. In Tabelle 12 sind für einen beispielhaften Vergleich die fünf Hauptbaumarten mit ihren jeweiligen Holzproduktionswerten einer vollständigen Umtriebszeit dargestellt. Dabei wurden für jedes Kalkulationstool die gleichen, in der Tabelle beschriebenen, naturalen Parameter gewählt. Bei der Fichte zeigt sich die stärkste Abweichung (-24\%) zwischen dem Ergebnis auf Basis des niedersächsischen Tools im Vergleich mit dem Tool der Universität Göttingen. Auch bei der Eiche liegt der niedersächsische Wert (-43 \%) weit unter dem Ergebnis der Universität Göttingen. Umgekehrt sind die hessischen Werte für Douglasie (+15\%) und Buche (+31 \%) wesentlich höher als im Bewertungskonzept des Autors dieser Arbeit.

Tabelle 12: Vergleich Referenzbewertungsfälle der fünf Hauptbaumartengruppen mittels der drei Kalkulationstools, Abweichungen zu Bewertungskonzept der Uni Göttingen in \%.

\begin{tabular}{|c|c|c|c|c|}
\hline & & Hessen & Niedersachsen & Uni Gö/NRW \\
\hline \multirow[t]{2}{*}{$\underset{t}{\stackrel{0}{c}}$} & $\begin{array}{l}\text { Umtriebszeit=100, 1.Ekl. bzw. } 12 \text {. Lkl., durchschnittliche } \\
\text { Wertklasse, Kulturkosten } 1400 €(N V) \text {, Pflegekosten } 300 € \text { im } \\
\text { Alter } 20\end{array}$ & $256 € / \mathrm{ha} / \mathrm{a}$ & $207 € /$ ha/a & $274 € /$ ha/a \\
\hline & Verhältnis zu Uni Gö & $-7 \%$ & $-24 \%$ & \\
\hline \multirow[t]{2}{*}{$\frac{\grave{d}}{\frac{d}{2}}$} & $\begin{array}{l}\text { Umtriebszeit }=120,1 . E k l . \text { bzw. } 8 \text {. Lkl., durchschnittliche } \\
\text { Wertklasse, Kulturkosten } 1100 €(N V) \text {, Pflegekosten } 400 € \text { im } \\
\text { Alter } 20\end{array}$ & $96 € /$ ha/a & $90 € /$ ha/a & $94 € /$ ha/a \\
\hline & Verhältnis zu Uni Gö & $2 \%$ & $-4 \%$ & \\
\hline \multirow[t]{2}{*}{$\begin{array}{l}\frac{0}{5} \\
\frac{\pi}{00} \\
\frac{0}{00}\end{array}$} & $\begin{array}{l}\text { Umtriebszeit }=80,1 . E k l \text { bzw. } 17 \text { Lkl., durchschnittliche } \\
\text { Wertklasse, Kulturkosten } 1700 €(N V) \text {, Pflegekosten } 600 € \mathrm{im} \\
\text { Alter } 20\end{array}$ & $479 € /$ ha/a & $390 € /$ ha/a & $416 € / \mathrm{ha} / \mathrm{a}$ \\
\hline & Verhältnis zu Uni Gö & $15 \%$ & $-6 \%$ & \\
\hline \multirow[t]{2}{*}{ 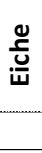 } & $\begin{array}{l}\text { Umtriebszeit=160, 1.Ekl bzw. } 7 \text { Lkl., durchschnittliche } \\
\text { Wertklasse, Kulturkosten } 3100 €(N V) \text {, Pflegekosten } 600 € \text { im } \\
\text { Alter } 20\end{array}$ & $95 € /$ ha/a & $69 € /$ ha/a & $122 € / \mathrm{ha} / \mathrm{a}$ \\
\hline & Verhältnis zu Uni Gö & $-22 \%$ & $-43 \%$ & \\
\hline \multirow[t]{2}{*}{ 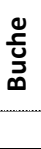 } & $\begin{array}{l}\text { Umtriebszeit=120, 1.Ekl. / } 9 \text { Lkl., durchschnittliche } \\
\text { Wertklasse, Kulturkosten } 2.800 € \text {, Pflegekosten } 250 € \text { im Alter } \\
20\end{array}$ & $77 € /$ ha/a & $67 € /$ ha/a & $59 € /$ ha/a \\
\hline & Verhältnis zu Uni Gö & $31 \%$ & $14 \%$ & \\
\hline
\end{tabular}

Die dargestellten Abweichungen resultieren aus einer Kombination von geringeren Holzerlösen bei gleichzeitig geringeren Holzvorräten, die aus der Anpassung der Ertragstafeln an niedersächsische Wuchsverhältnisse resultieren. 


\subsubsection{Fazit}

Die vorliegenden drei Bewertungstools sind alle zum gleichen Zweck auf Basis des in Kapitel 4.3.1 vorgestellten methodischen Ansatzes erstellt worden. Unter Berücksichtigung der regionalen Verwendung wurden die Basisdaten auf Grundlage der geltenden Waldbewertungsrichtlinien hinterlegt. Daraus resultierende Unterschiede lassen sich für vergleichbare Bewertungsfälle darstellen. Allen drei Tools ist gemeinsam, dass sie in der Anwendung stets einer fallspezifischen Anpassung an die Realität bedürfen.

\subsubsection{Aktualisierung Bewertungskonzept für Einzelbäume}

\subsubsection{Ziel}

Das Bewertungskonzept für Einzelbäume ist vom Deutschen Forstwirtschaftsrat (DFWR) im Jahr 2010 erstmals veröffentlicht worden (Möhring 2010). Hintergrund ist die Bereitstellung eines Bewertungstools für die Praxis auf Grundlage der Abtriebswerttabellen für Einzelbäume aus der niedersächsischen Waldbewertungsrichtlinie (WBR NDS) von 2008, mithilfe dessen die Bewertung von einzelnen Bäumen bspw. zum Zwecke des Erhalts von Alt- und Habitatbäumen ermöglicht wird.

Aufgrund der Veränderungen am Holzmarkt und der Aktualisierung der niedersächsischen Waldbewertungsrichtlinie bedurften vor allem die Abtriebswerte der Einzelbäume einer Anpassung an das aktuelle Niveau.

\subsubsection{Methodik}

Ebenso wie das Bewertungskonzept für forstliche Nutzungsbeschränkungen (s. 4.3.1) baut das Bewertungskonzept für Einzelbäume auf dem Ertragswertkonzept auf (Möhring 2010, S. 11). Gegenstand der Bewertung sind „hiebsreife Einzelbäume“, die sich nach betrieblicher Zielsetzung in der Erntephase befinden und planmäßig innerhalb des Bewertungszeitraums eingeschlagen würden. Die Ertragsbewertung erfolgt als analytischer Ansatz, der Gesamtwert setzt sich als Summe der Teilkomponenten der Bewertung zusammen. Zur besseren Nachvollziehbarkeit erfolgt eine Ermittlung der jährlichen Werte in Euro pro Baum. Bei Bedarf können diese als Barwerte zum Periodenanfang umgerechnet werden (Möhring 2010, S. 11).

Der Gesamtwert eines Baumes setzt sich aus folgenden Komponenten (s. Tabelle 13) zusammen:

1. Verzicht auf den Einschlag mit der resultierenden Realisierung des Abtriebswerts berechnet als jährlicher Zinsverlust in €/Baum. 
2. Berücksichtigung des Wertverlusts durch Entwertung oder Marktveränderungen, berechnet als relative Wertveränderung zwischen Beginn und Ende des Bewertungszeitraums, dargestellt als Annuität des jährlichen Wertverlusts.

3. Ertragsentgang des Folgebestands durch die weiterhin überschirmte Produktionsfläche, dargestellt als Bodenbruttorente aus der Waldbewertungsrichtlinie des geplanten Folgebestands.

4. Die Kosten der Auswahl/Markierung und der sonstigen Erschwernisse, insbesondere bei Vertragsnaturschutzprogrammen, werden aus der Analyse des Zeitbedarfs ermittelt (s. 2.5).

5. Der Preis eines Gutes muss stets seine Kosten übersteigen, um angeboten zu werden. Daher wird ein Vertragsabschluss- oder Anreizfaktor als prozentualer Zuschlag berücksichtigt.

Die Aktualisierung der Daten und daraus resultierende Ergebnisse werden nachfolgend dargestellt.

\subsubsection{Aktualisierung der Daten}

In der vorliegenden Aktualisierung wurden die zugrundliegenden Abtriebswerttabellen der Einzelbäume aus der WBR NDS von 2008 durch die aktualisierten der WBR NDS in der Fassung von 2014 ersetzt. Da die Abtriebswerttabellen der Niedersächsischen Landesforsten (NLF) im Laubund Nadelholz nur bis zu einem BHD von $60 \mathrm{~cm}$ bzw. maximal $72 \mathrm{~cm}$ reichen, für die Kalkulation von starken Einzelbäumen jedoch auch höhere BHD berechnet werden müssen, wurde, wie bei der ersten Version auch, eine Extrapolation der Abtriebswerte in den hohen BHD-Bereichen durchgeführt. Bei der Modellierung der hohen BHD-Klassen zeigte sich, dass eine lineare Extrapolation durch die drei stärksten gegebenen BHD der WBR die plausibelsten Ergebnisse zeigte (s. Abbildung 40). Andere Funktionsverläufe führten zu unplausiblen oder nicht interpretierbaren Wertverläufen. Der neuen Skalierung der NLF der BHD in 4cm-Schritten wurde durch eine Anpassung der Kalkulationsformeln Rechnung getragen. 


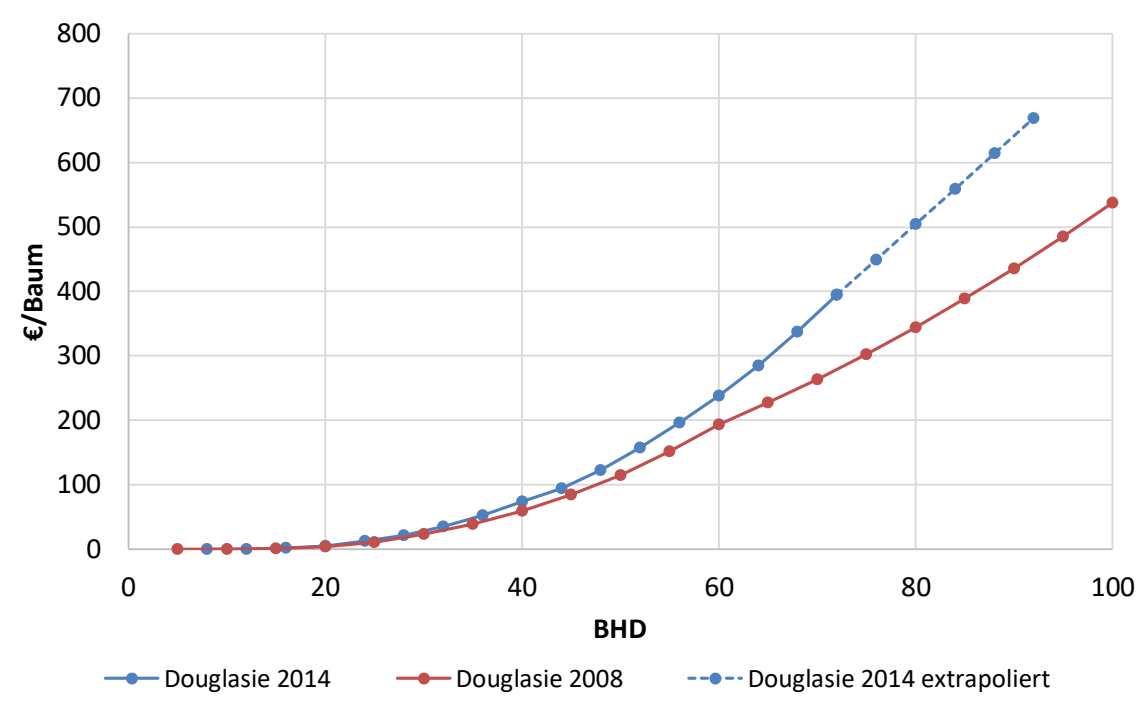

Abbildung 40: Abtriebswert in Abhängigkeit des BHD inkl. Extrapolation hoher BHD-Stufen bei Douglasie 2014

Neben dem reinen Holzwert des stehenden Baumes ist auch die von ihm überschirmte Fläche als Grundlage zur Bewertung des verhinderten Nachfolgebestandes wichtiger Bestandteil des Bewertungskonzepts (Opportunitätskosten der Flächeninanspruchnahme). Die Parameter zur Berechnung dieser Kronenschirmflächen bleiben unverändert (Nagel 2009). Hier liegt keine neue Datengrundlage vor. Die Bodenbruttorente zur Bewertung der Kronenschirmfläche bleibt ebenfalls unverändert, da in der WBR NDS 2014 weiterhin durchschnittlich $165 €$ /ha/a verwendet werden (Niedersächsisches Ministerium für Landwirtschaft und Ernährung 2014).

Die für dieses Konzept erforderliche Größe der sonstigen Erschwernisse wurde im Rahmen des WaVerNa-Projekts überprüft. So konnten in den Fallbeispielsanalysen für Dokumentation, Umsetzung und Kontrolle des Erhalts von Alt- und Habitatbäumen durchschnittlich $24 €$ /Baum Aufwand ermittelt werden (Blomberg und Möhring 2018). Es ist davon auszugehen, dass diese Arbeiten durchschnittlich alle vier Jahre erforderlich sind. Der anzusetzende Betrag beläuft sich daher auf $6 € /$ Baum/Jahr (Blomberg und Möhring 2018).

\subsubsection{Veränderung des In- und Outputs}

Die Aktualisierung der Abtriebswerttabellen führt zu wesentlichen Veränderungen der Abtriebswertverläufe. Für die Laubhölzer Eiche, Buche und Esche zeigt sich in der beispielhaft ausgewählten Wertklasse 2 besonders in höheren BHD-Bereichen eine Abnahme der Abtriebswerte (s. Abbildung 41). Bei der Eiche liegt der aktualisierte Abtriebswert ab dem BHD von $36 \mathrm{~cm}$ unter dem Abtriebswert der Version von 2010, bei der Buche ist es ab einem BHD von $60 \mathrm{~cm}$ der Fall. Bei Betrachtung der Abtriebswertverläufe über die Wertklassen 2 bis 4 zeigt sich bei der Eiche eine Abnahme in allen Wertklassen. Die Abtriebswerte der Wertklassen 2 und 3 der Buche liegen 
ebenfalls unter den vormaligen Werten, die Wertklasse 4 hingegen kann gegenüber 2008 eine Verbesserung verzeichnen.

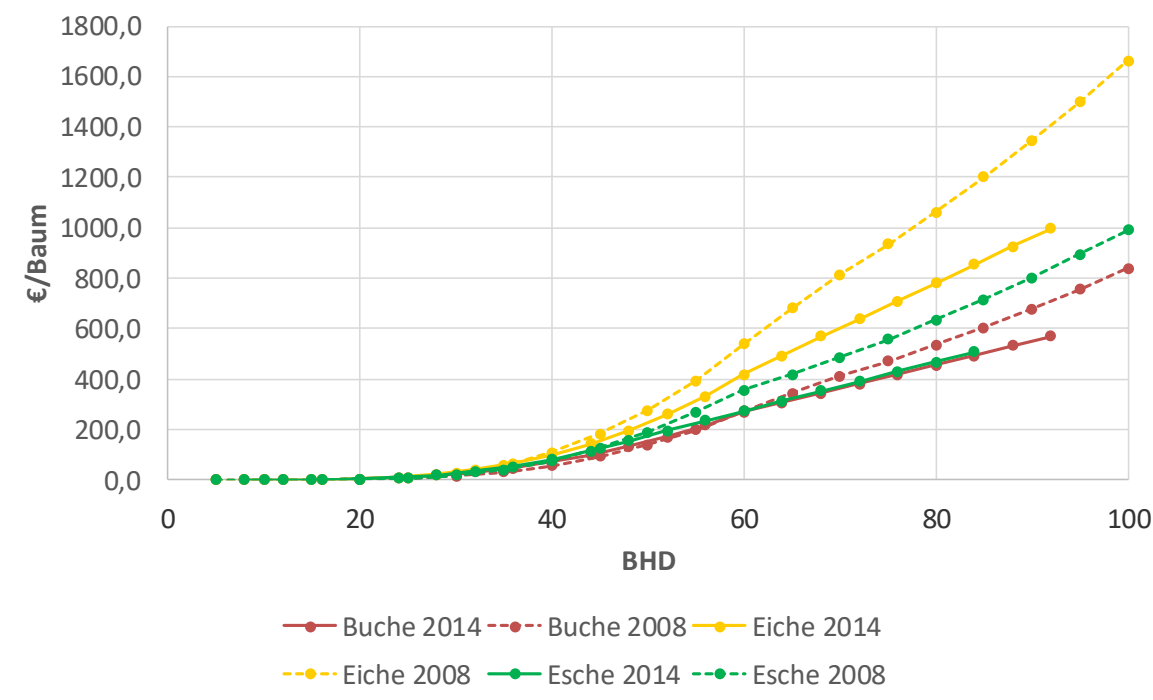

Abbildung 41: Veränderung der Einzelbaumabtriebswerte in Abhängigkeit vom BHD der Laubbaumarten, 2008 (gestrichelt) zu 2014 (durchgezogen). Wertklasse 2

Bei den vier betrachteten Nadelbaumarten ist die Situation etwas differenzierter. Bei den Baumarten Fichte, Douglasie und Lärche liegen die neuen Abtriebswerte ab einem BHD von $20 \mathrm{~cm}$ stets über den alten. Diese Entwicklung ist über alle Wertklassen gleich.

Bei der Kiefer liegen die neuen Werte der Abtriebswerttabellen ebenfalls über den alten. Die Extrapolation der nichtgegebenen Werte führt jedoch zu einem geringeren Anstieg als bei den alten. Daher liegen ab einem BHD von $75 \mathrm{~cm}$ die neuen Werte wieder unter den alten. Dies gilt für alle drei betrachteten Wertklassen.

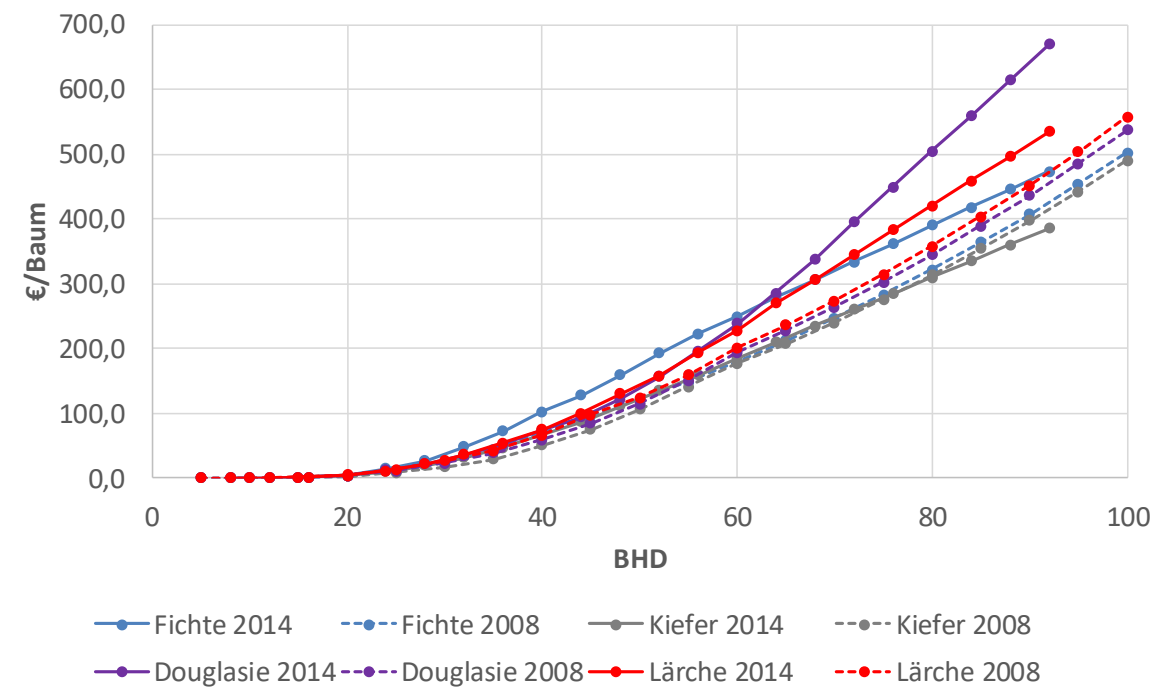

Abbildung 42: Veränderung der Einzelbaumabtriebswerte in Abhängigkeit vom BHD der Nadelbaumarten, 2008 (gestrichelt) zu 2014 (durchgezogen). Wertklasse 2 
Wie im aktualisierten Bewertungskonzept für forstliche Nutzungsbeschränkungen ist auch in Abbildung 43 die Veränderung von 2010 zu 2018 entlang der Winkelhalbierenden aufgetragen (vgl. Möhring et al. 2017). Deutlich zu erkennen ist die oben bereits angesprochene Verschlechterung der Abtriebswerte für die Laubbaumarten Buche und Eiche, während die Nadelbaumarten Fichte und Douglasie eine Verbesserung erfahren haben. Die Kiefer liegt aufgrund der kaum vorhandenen Veränderung annähernd auf der Winkelhalbierenden.

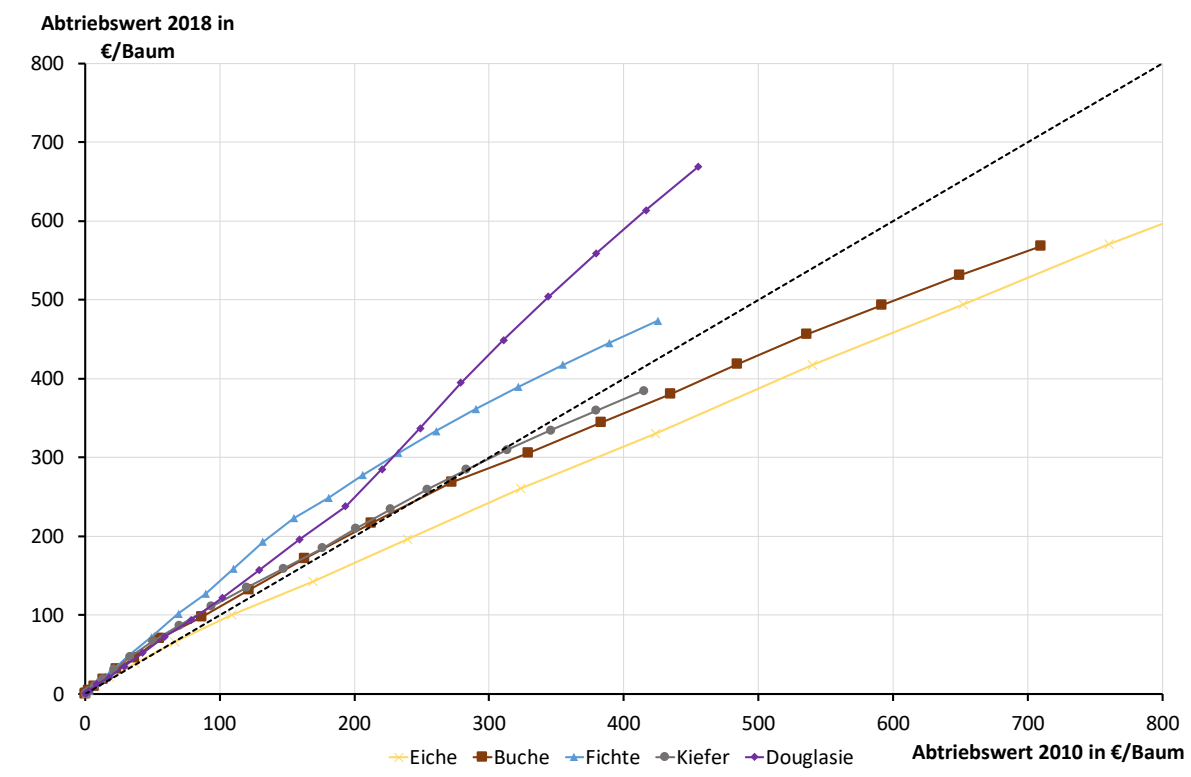

Abbildung 43: Vergleich der Abtriebswerte in €/Baum 2010 zu 2018 für die Hauptbaumarten Wkl. 2. Werte auf der schwarz gestrichelten Winkelhalbierenden bedeuten keine Veränderung, Werte oberhalb eine Zunahme, Werte unterhalb eine Abnahme der Abtriebswerte.

\subsection{Anwendungsbeispiel}

In Tabelle 13 sind beispielhaft die gleiche Reihe von Einzelbäumen verschiedener Baumarten mit variierenden Wertklassen wie schon in der ersten Version von 2010 auf Basis der erläuterten Eingangsgrößen berechnet worden. Die gewählten 10 Bäume verursachen jährliche Gesamtkosten von 133,44€ ohne Berücksichtigung eines Vertragsabschlussfaktors (Anreizkomponente) und 160,13€ inklusive eines 20-prozentigen Aufschlags. Als Barwert für eine einmalige Vergütung des Erhalts dieser Bäume über einen Zeitraum von 20 Jahren wären 2.749,15€ notwendig. Im Vergleich zu den ursprünglichen Werten, die in Tabelle 13 in orange hinterlegt sind, zeigen sich je nach gewähltem Bewertungsbeispiel unterschiedliche Veränderungen. So hat bspw. die Eiche mit der Wertklasse 2 -19\% Wert gegenüber 2010 eingebüßt, wohingegen der Wert der Douglasie um 18 \% gestiegen ist. Diese Wertveränderungen, resultierend aus den angepassten Abtriebswerttabellen, decken sich entsprechend mit den in 4.3.3.3 gezeigten Verschiebungen. 
Maßgeblichen Einfluss auf den Gesamtwert haben auch die jährlichen Zinskosten aus der Bindung des Kapitals im Abtriebswert sowie die sonstigen jährlichen Erschwernisse (Verwaltungsund Kontrollaufwand).

Die Kalkulationsvorgaben sind jederzeit an einen konkreten Bewertungsfall anpassbar.

Tabelle 13: Bewertungskonzept für Einzelbäume, beispielhafte Berechnung einiger Baumarten mit variierenden Wertklassen (blau) inkl. Vergleich zu den ermittelten Barwerten je Baum aus 2010 (orange) und Abweichung in \% (grün). Zur besseren Lesbarkeit befindet sich die Tabelle erneut im Anhang 9.1.4.

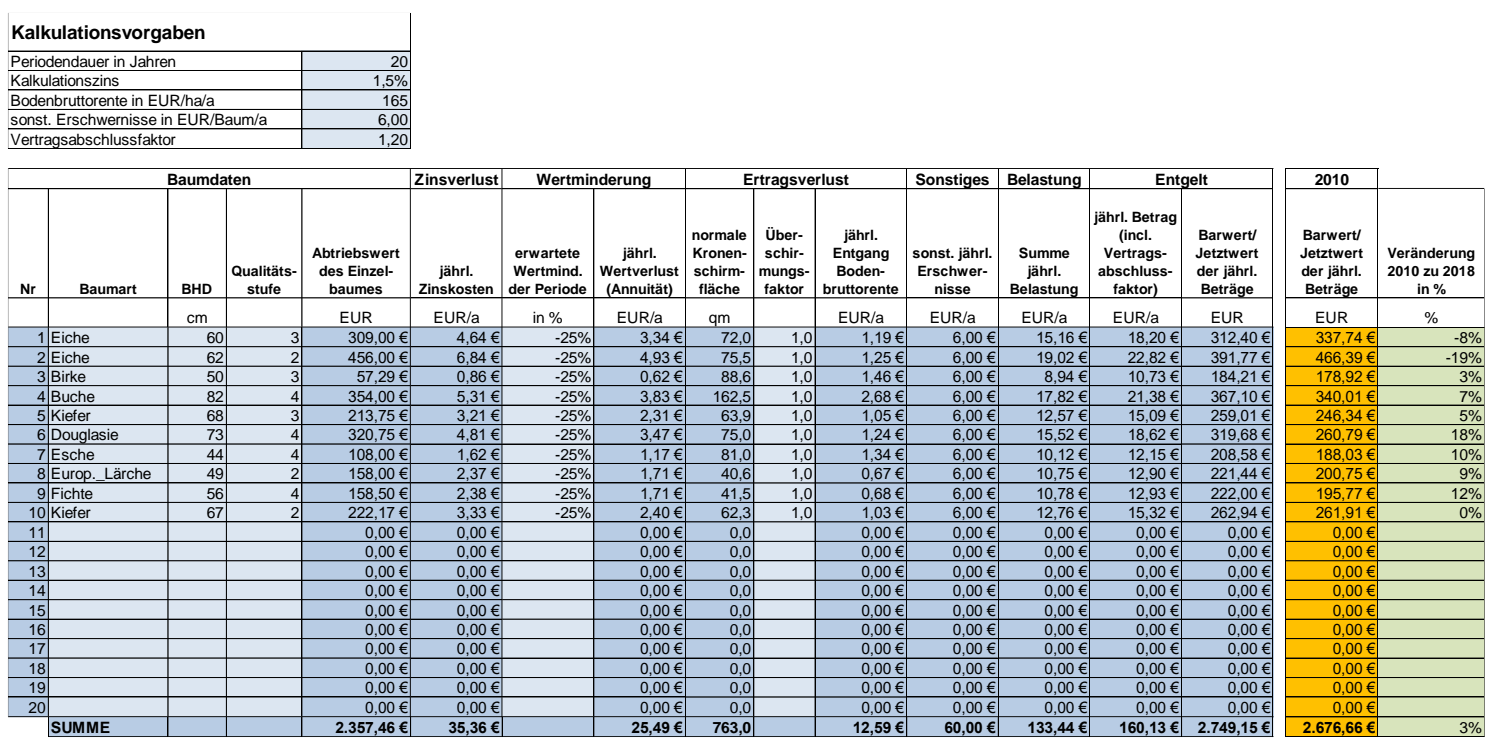

Neben den Veränderungen der Einzelbaumabtriebswerte, die aus der Aktualisierung der Eingangsdaten resultieren, haben auch die sonstigen unterstellten Parameter wesentlichen Einfluss auf die berechneten Werte. Im Bewertungskonzept werden neben dem reinen Holzwert des Baumes auch die sonstigen Erschwernisse und Kosten berücksichtigt. Der Gesamtwert, ausgedrückt wahlweise als jährlicher Betrag oder diskontiert als Barwert des Betrachtungszeitraums, gibt Auskunft über die Höhe der betrieblichen Belastung. Dessen Veränderlichkeit wird durch die Variation nachfolgender Parameter untersucht:
a) Wertklasse
b) Wertverlust während der Laufzeit

Die Eingangsdaten für die Bodenbruttorente, die sonstigen Erschwernisse und den Vertragsabschlussfaktor werden an dieser Stelle nicht dargestellt, da eine Veränderung dieser Werte lediglich zu einer Parallelverschiebung der Wertverläufe an der x-Achse führt. Sie werden in ihrem Wert festgelegt, so dass die Variation der Wertklasse und des Wertverlusts "ceteris paribus" stattfindet. 
In Abbildung 44 ist für die Baumart Buche die Veränderung der Barwerte in Abhängigkeit der Wertklassen und des BHD dargestellt. Mit zunehmender Stärke eines Baumes erhöht sich der Abtriebswert in höheren Wertklassen überproportional und die Zinseffekte des gebundenen Kapitals wirken sich stärker aus.

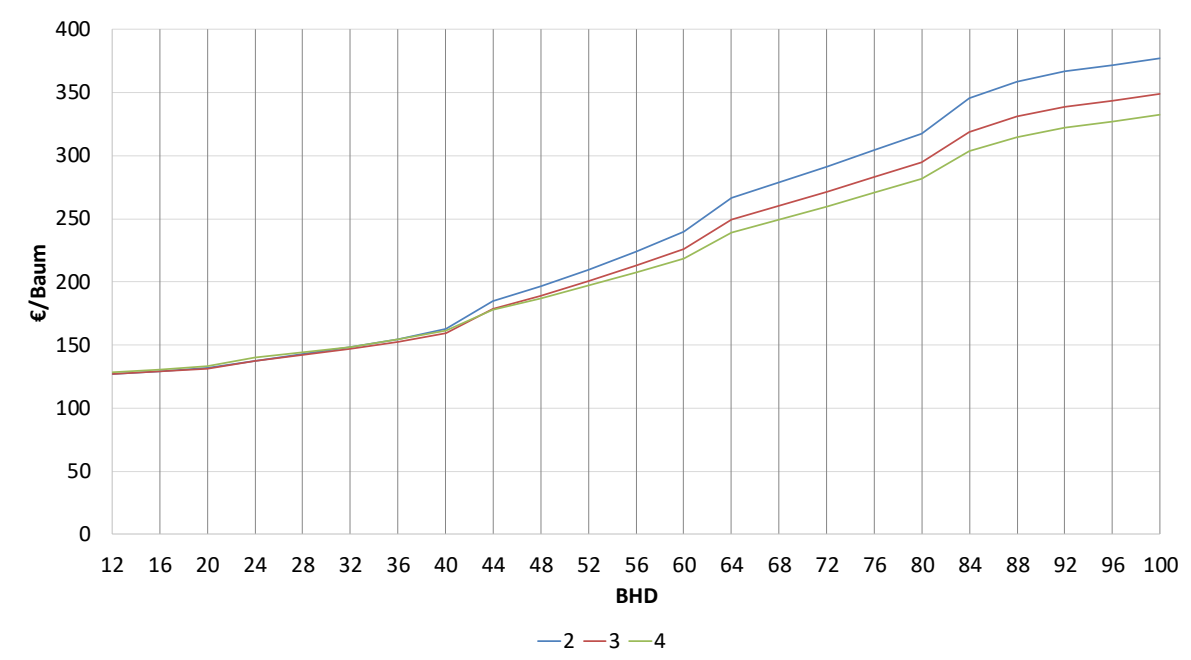

Abbildung 44: Vergleich der Barwerte Baumart Buche nach Wertklassen in Abhängigkeit vom BHD, sonstige Eingangsdaten unverändert, die Sprünge in den Barwertverläufen resultieren aus Sprüngen in den Einzelbaumabtriebswerten

Im Gegensatz zur vorhergehenden Abbildung wurde in Abbildung 45 die Wertklasse (WKL) 2 festgesetzt, dafür jedoch der unterstellte Wertverlust des stehenden Baumes im Betrachtungszeitraum von $-0 \%$ bis $-100 \%$ variiert. Der Kurvenverlauf ist geprägt durch die unterstellten Abtriebswerte, jedoch zeigt sich durch die zusätzlichen Kosten der Entwertung eine deutliche Anhebung der Barwerte mit zunehmendem BHD. Je stärker und damit wertvoller ein Einzelbaum ist, desto stärker wirkt sich auch die Entwertung auf den Barwert aus.

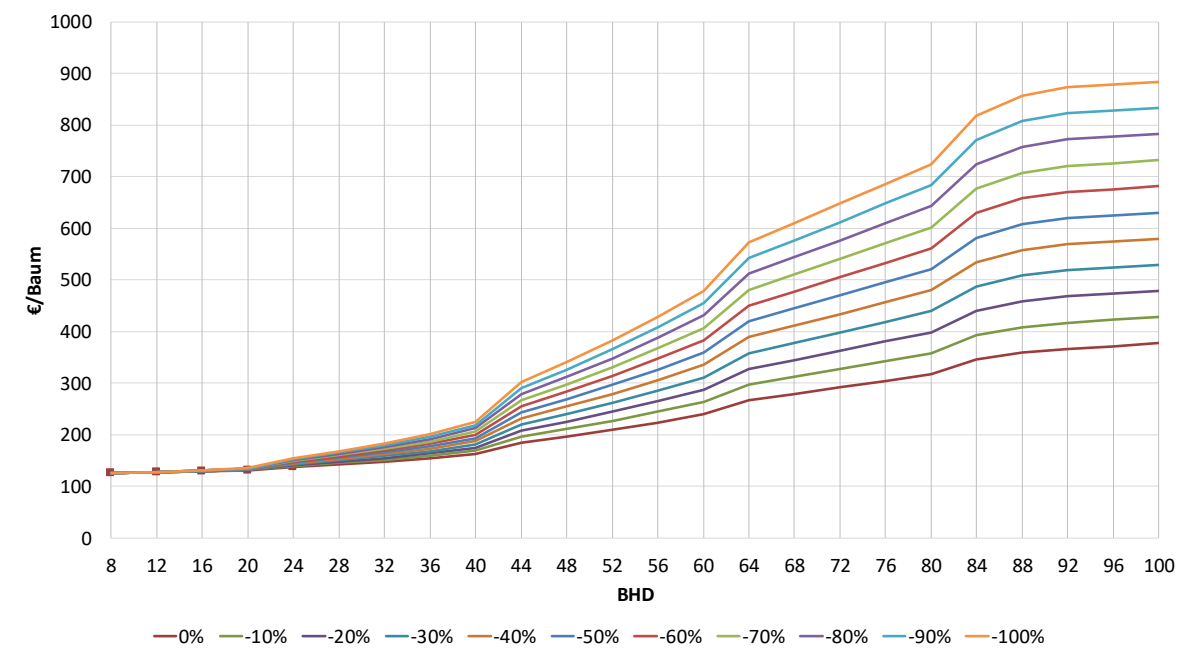

Abbildung 45: Barwerte der Einzelbäume Buche, Wkl. 2 in Abhängigkeit des unterstellten Wertverlusts über die Laufzeit, sonstige Eingangsdaten unverändert 
Besonders der absolute Vergleich zwischen der Variation der Wertklasse und dem unterstellten Wertverlust zeigt deutlich die ökonomisch relevanten Auswirkungen auf. Während bei der oben beispielhaft gewählten Buche bei einem BHD von $80 \mathrm{~cm}$ der Unterschied zwischen WKL 2 (318 €/Baum) und WKL 4 (282 €/Baum) nur $36 €$, bzw. -11 \% beträgt, liegt die Differenz zwischen keinem Wertverlust und der vollständigen Entwertung bei $318 € /$ Baum bzw. $724 € /$ Baum. Dies entspricht einer Zunahme um 228 \% (vgl. Abbildung 44 \& Abbildung 45).

\subsubsection{Zusammenfassung Einzelbaumbewertung}

Das Bewertungskonzept für Einzelbäume bedurfte rund 10 Jahre nach seiner ursprünglichen Veröffentlichung einer Anpassung an die veränderte Situation im Wald und an den Holzmärkten. Die Preisgefüge beim Laub- und beim Nadelholz haben sich gegensätzlich verschoben, so dass die ökonomische Vorteilhaftigkeit von Nadelhölzern noch stärker zu Tage tritt. Für bestehende Vertragsnaturschutzangebote bedeutet dies, dass sie einer Überprüfung hinsichtlich der preislichen Angemessenheit bedürfen. Mit der vorgenommenen Aktualisierung der zugrunde liegenden Daten des Bewertungskonzepts für Einzelbäume liegt nun ein in der Praxis für Bewertungsfälle anwendbares Handwerkszeug vor, dass gerade bei der wichtigen Maßnahme des Erhalts von Alt- und Habitatbäumen den Beteiligten ein transparentes Instrument zur Bewertung und Preisfindung bietet.

\subsection{Fallstudie Hämeler Wald}

Die im vorangegangenen Kapitel beschriebenen Konzepte zur Bewertung der ökonomischen und organisatorischen Erschwernisse durch Naturschutzauflagen im Wald wurden in einer Fallstudie der praktischen Anwendung unterzogen, die nachfolgend dargestellt werden soll.

Hintergrund war die Analyse der Auswirkungen der niedersächsischen Verordnung der "Unterschutzstellung von Natura 2000-Gebieten im Wald durch Naturschutzgebietsverordnung" (Niedersächsisches Ministerium für Umwelt, Energie und Klimaschutz 2015) auf ein konkretes FFH-Gebiet. Der Niedersächsische Waldbesitzerverband hatte die Abteilung Forstökonomie der Universität Göttingen um diese Analyse gebeten, um eine Informationsveranstaltung für Mitglieder und Vertreter der Politik durchzuführen. Die Natura 2000-Gebiete sind Gebiete, die auf Grundlage der Flora-Fauna-Habitat-Richtlinie ${ }^{38}$ und der Europäischen Vogelschutzrichtlinie ${ }^{39}$ der Europäischen Union zu geschützten Teilen von Natur und Landschaft zu erklären sind. Das Land Niedersachsen stellt Wald im Sinne des §2 NWaldLG nach §32 Abs. 2 und 3 BNatSchG durch

\footnotetext{
${ }^{38}$ Richtlinie 92/43/EWG (FFH-Richtlinie)

${ }^{39}$ Richtlinie 2009/147/EG (Vogelschutz-Richtlinie)
} 
Naturschutzgebietsverordnung unter Schutz, sofern dort wertbestimmende Lebensraumtypen oder Arten vorkommen ${ }^{40}$

Die Unterschutzstellung mittels Naturschutzgebietsverordnung (oder Landschaftsschutzgebietsverordnung, wenn zur Schutzzweckerreichung ausreichend) beinhaltet für die Lebensraumtypflächen einen Kanon von Bewirtschaftungsauflagen und -einschränkungen, die nach Einschätzung der betroffenen Waldbesitzer zu wirtschaftlichen Einbußen führen. Zur Quantifizierung der Ertragseinbußen wurde am Beispiel des Hämeler Walds in Kooperation mit dem Waldbesitzerverband Niedersachsen diese Fallstudie durchgeführt. Auf drei Probeflächen in wertbestimmenden Lebensraumtypflächen wurden die zuvor beschriebenen Bewertungskonzepte auf die naturschutzbedingten Einschränkungen angewendet, um auf diesen Einzelfall bezogen die ökonomischen Auswirkungen der Betroffenen darzustellen.

Für die vorliegende Arbeit diente die Fallstudie als exemplarische Anwendung der im Vorfeld aktualisierten und erweiterten Bewertungstools. Besonders unter dem Aspekt der Kommunikationsunterstützung der Beteiligten durch die Bewertungstools, kann mittels nachvollziehbar ermittelter Zahlen eine transparentere Gesprächsgrundlage geschaffen werden.
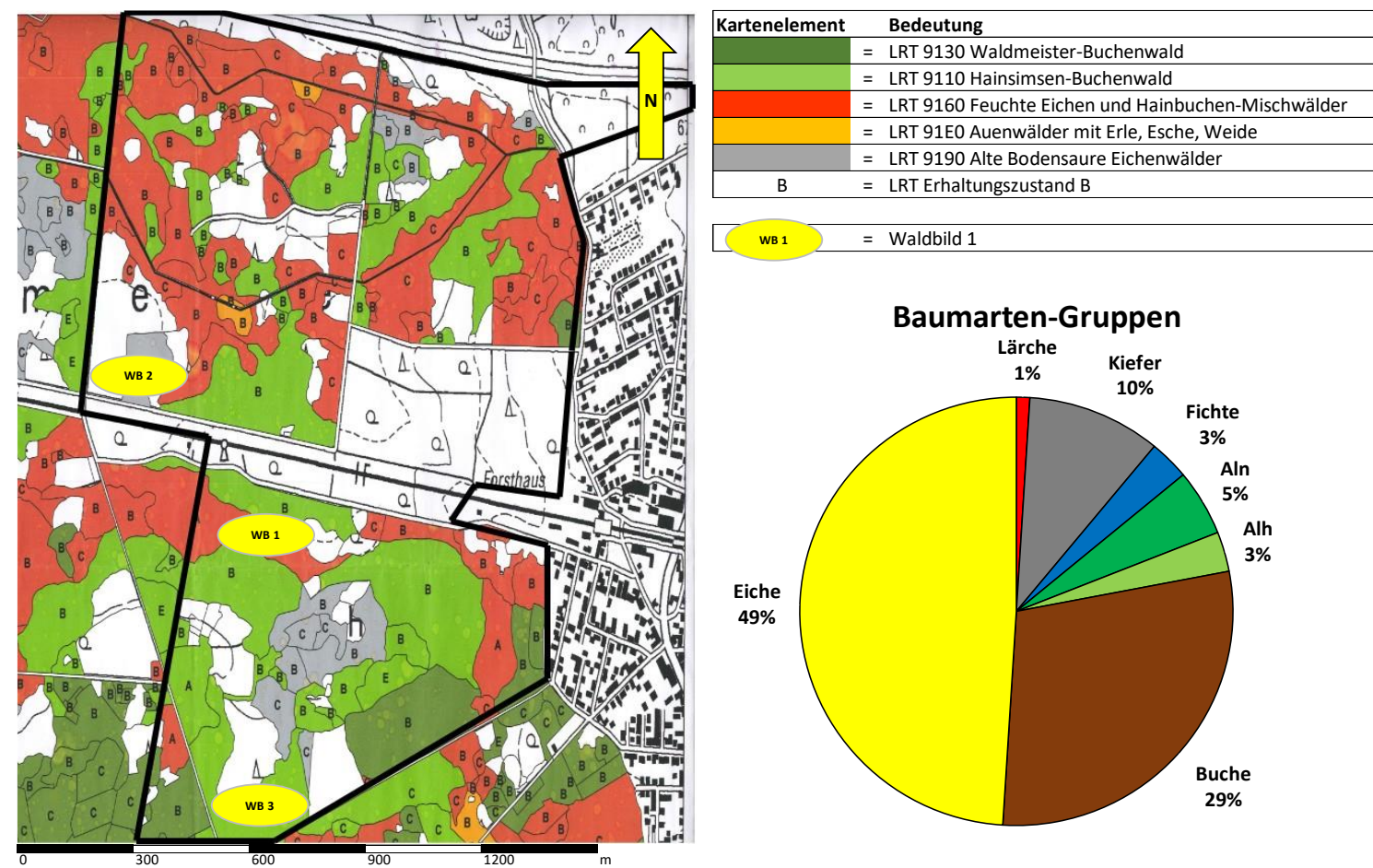

Abbildung 46: Fallstudie Hämeler Wald mit Lebensraumtypflächen, Erhaltungszuständen, Probeflächen und Baumartenverteilung

\footnotetext{
${ }^{40}$ Gemeinhin als FFH-Gebiet mit Lebensraumtypflächen (LRT) bezeichnet.
} 
Zum Bewertungszeitpunkt im September 2017 war die Unterschutzstellung des betroffenen Gebiets noch nicht erfolgt, insofern diente die Ermittlung der Erschwernisse als vorweggenommene Folgenabschätzung.

Die aufzustellende Schutzgebietsverordnung umfasste neben allgemeinen Beschränkungen auf allen Flächen mit wertbestimmenden Lebensraumtypen (LRT) wie z.B. Düngungsverbot, jahreszeitlichen Einschlagsbeschränkungen in Althölzern oder weiten Gassenabständen auch spezifische Regelungen in Abhängigkeit der ausgewiesenen Erhaltungszustände (EZ) (s. Tabelle 14). Besondere Bedeutung für die weitere Bewirtschaftung der Bestände haben dabei der Erhalt von drei (LRT EZ B/C) bzw. sechs (LRT EZ A) Habitatbäumen, der Altholzerhalt von 20 \% (LRT EZ B/C) bzW. $35 \%$ (LRT EZ A) auf der Lebensraumtypfläche und die Baumartenzusammensetzung der künstlichen Verjüngung, bei der 80 \% (LRT EZ B/C) bzw. 90 \% (LRT EZ A) lebensraumtypische Hauptbaumarten verwendet werden müssen. Verstärkt wird der letzte Punkt noch durch die Zielbestimmung der Baumartenzusammensetzung, die im Erhaltungszustand B/C auf $80 \%$ und $90 \%$ bei Erhaltungszustand A der LRT-Fläche lebensraumtypische Baumarten umfassen muss.

Tabelle 14: Auflagen der niedersächsischen Natura 2000-Schutzgebietsverordnung im Wald für die Erhaltungszustände, gekürzt, eigene Darstellung

\begin{tabular}{|c|c|c|}
\hline & Erhaltungszustand $\mathrm{A}$ & Erhaltungszustand B/C \\
\hline Habitatbaumerhalt & 6 je ha LRT & 3 je ha LRT \\
\hline alternativ & & $\begin{array}{l}\text { ab dem dritten Eingriff } 5 \% \text { Flächenstilllegung zur } \\
\text { Entwicklung von Habitatbaumanwärtern }\end{array}$ \\
\hline Altholzerhalt & $35 \%$ der LRT-Fläche pro Eigentümer & 20 \% der LRT-Fläche pro Eigentümer \\
\hline Künstliche Verjüngung & $\begin{array}{l}\text { Ausschließlich lebensraumtypisch und auf } 90 \% \text { der LRT- } \\
\text { Fläche lebensraumtypische Hauptbaumarten }\end{array}$ & $\begin{array}{l}\text { Ausschließlich lebensraumtypisch und auf } 80 \% \text { der LRT- } \\
\text { Fläche lebensraumtypische Hauptbaumarten ODER } \\
\text { mindestens } 90 \% \text { lebensraumtypische Baumarten }\end{array}$ \\
\hline Baumartenzusammensetzung & $\begin{array}{l}\text { Erhalt und Entwicklung von lebensraumtypischen } \\
\text { Baumarten auf } 90 \% \text { der LRT-Fläche }\end{array}$ & $\begin{array}{l}\text { Erhalt und Entwicklung von lebensraumtypischen } \\
\text { Baumarten auf } 80 \% \text { der LRT-Fläche }\end{array}$ \\
\hline Totholz, stehend oder liegend & 3 Stück je Hektar LRT-Fläche & 2 Stück je Hektar LRT-Fläche \\
\hline Gassenabstand im Altholz & $40 \mathrm{~m}$ & $40 \mathrm{~m}$ \\
\hline Sonstiges & \multicolumn{2}{|c|}{$\begin{array}{l}\text { Kahlschlagsverbot, Befahrungsverbot, Herbizidverbot, Düngungsverbot, } \\
\text { Keine Maßnahmen vom 1. März bis 31. August ohne Genehmigung der UNB }\end{array}$} \\
\hline
\end{tabular}

Weitere Maßnahmen wie Wegeinstandsetzung oder Holzeinschlag im Sommerhalbjahr sind zwar grundsätzlich weiter möglich, bedürfen jedoch der Mitteilung an oder Genehmigung durch die zuständigen Unteren Naturschutzbehörden.

Die Anwendung der theoretischen Konzepte zur Bewertung von naturschutzbedingten Bewirtschaftungserschwernisse (s. Kapitel 4.1) erfolgte für folgende drei Maßnahmentypen, die die höchsten Einschränkungen mit sich bringen werden:

1. Habitatbaumerhalt von drei, bzw. sechs Habitatbäumen je Hektar Lebensraumtypfläche

2. Einschränkung der Baumartenwahl auf Lebensraumtypflächen durch $90 \%$ Anteil Hauptbaumarten in der Verjüngung 
3. Verlängerung der Umtriebszeit durch Erhalt von Altbeständen über den optimalen Erntezeitpunkt hinaus

\subsubsection{Methodik}

In der Fallstudie wurden drei Probeflächen angelegt, um die einzelnen Bewirtschaftungseinschränkungen einzeln zu erfassen und bewerten zu können. Die Probeflächen 1 und 3 lagen dabei in Altholzbeständen mit geringeren Stammzahlen, so dass der Probeflächenradius auf 32,75 m festgesetzt wurde. Daraus ergibt sich eine Fläche von 0,33 Hektar. Probefläche 2 lag in einem jüngeren Bestand mit höherer Stammzahl, so dass der Radius auf 28,21 m (entspricht 0,25 Hektar) festgelegt wurde. Beide Varianten ließen sich leicht und nachvollziehbar auf hektarbezogene Größen hochrechnen.

Für die Bewertung der Auswirkungen des Habitatbaumerhalts wurde das aktualisierte Konzept zur Bewertung von Einzelbäumen (s. Kapitel 4.3.3) verwendet. Eingang in die Bewertung fanden dabei zum einen die Kosten des Erhalts des Einzelbaumes sowie die aus dem Erhalt resultierenden Erschwernisse in der Bewirtschaftung des verbleibenden Bestands. Die Bewertung des Baumartenwechsels durch Anpassung der Verjüngung erfolgte mittels des aktualisierten und erweiterten Bewertungskonzepts für forstliche Nutzungseinschränkungen (s. Kapitel 4.3.1.6.2). Neben einer ökonomisch optimalen Baumartenwahl wurde die, auf Grundlage der Verordnung (Niedersächsisches Ministerium für Umwelt, Energie und Klimaschutz 2015), zu etablierende Bestandeszusammensetzung bewertet. Die Bewertung der Verlängerung der Umtriebszeit durch Erhalt hiebsreifer Altbestände erfolgte ebenfalls auf Grundlage des Bewertungskonzepts für forstliche Nutzungsbeschränkungen (s. Kapitel 4.3.1.6.4). Abschließend wurden diese Einzelbewertungspositionen für die Fallstudie Hämeler Wald zusammengeführt und die aus den drei wichtigsten Maßnahmentypen insgesamt resultierende Belastung ermittelt. 


\subsubsection{Habitatbaumerhalt}

Auf der ersten Probefläche, einem Buchen-Eichen-Mischbestand mit variierenden Dimensionen und Lebensraumtypfläche 9160 (Feuchte Eichen- und Hainbuchen-Mischwälder) mit Erhaltungszustand A wurden die Kosten des Habitatbaumerhalts und die Erschwernisse in der Bewirtschaftung durch den vorgeschriebenen Erhalt von Alt- und Habitatbäumen untersucht.

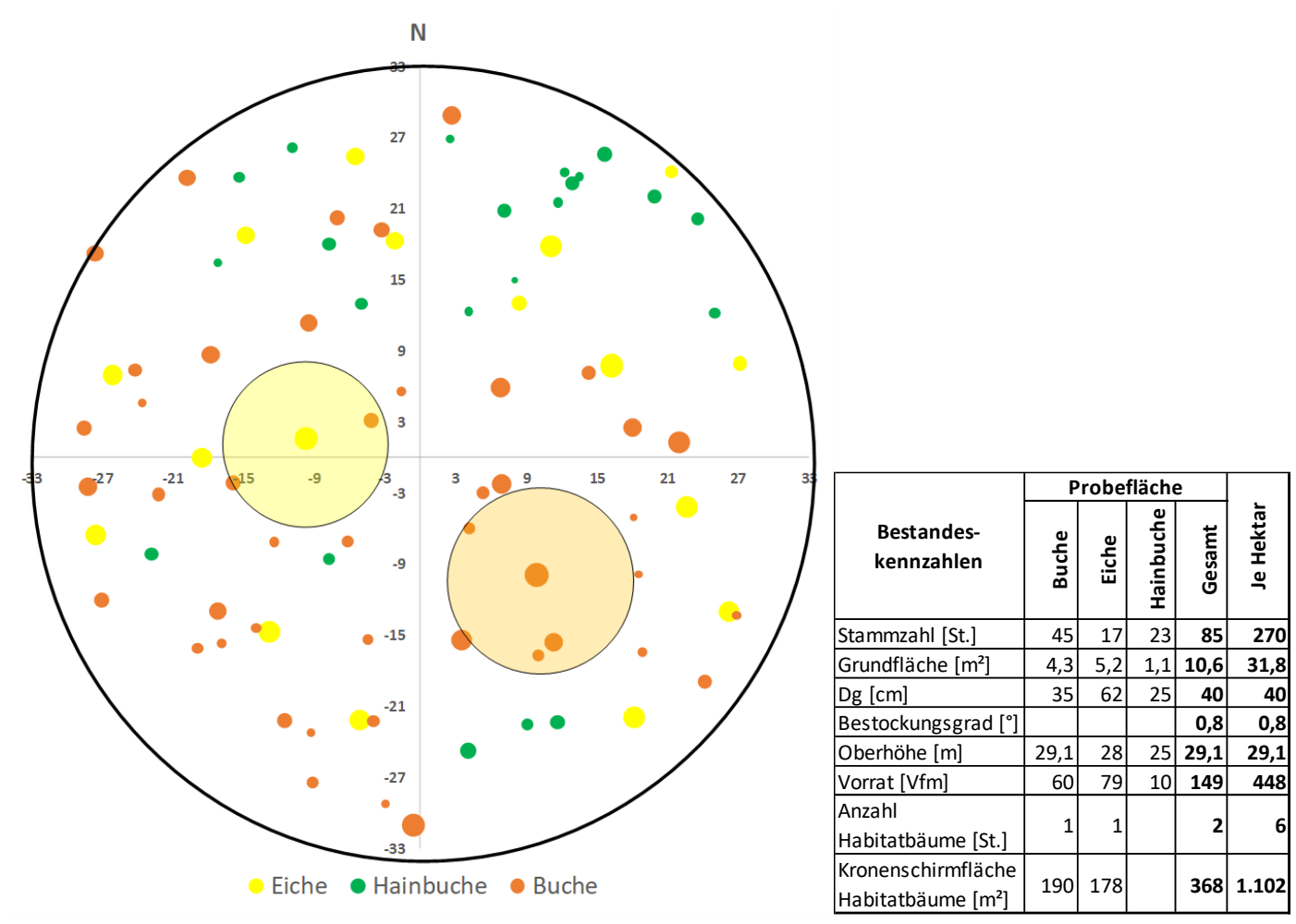

Abbildung 47: Stammverteilungsplan Probefläche 1 Hämeler Wald Abt. 1, Punktgröße in Abhängigkeit des Baumdurchmessers, Farbe in Abhängigkeit der Baumart

Mittels des Bewertungskonzepts für Einzelbäume wurden die auf der Probefläche vorkommenden Habitatbäume bewertet. Um der Schutzgebietsvorgabe von sechs Habitatbäumen je Hektar Lebensraumtypfläche im Erhaltungszustand gerecht zu werden, wurden für zwei habitatbaumgeeignete Einzelbäume die jährlichen Kosten des Erhalts berechnet. Aufgrund der hohen BHD von $73 \mathrm{~cm}$, bzw. $82 \mathrm{~cm}$ sind die ausgewählten Bäume der vorherrschenden Klasse zuzuordnen, das fortgeschrittene Alter und der biologische Zustand lassen eine vollständige ökonomische Entwertung in den nächsten 30 Jahren erwarten, so dass am Ende der Kalkulationsperiode keine Nutzung mehr möglich ist. Als Entgang der nachfolgenden Nutzung wird gem. Waldbewertungsrichtlinie Niedersachsen in der Fassung von 2014 (Niedersächsisches Ministerium für Landwirtschaft und Ernährung 2014) die durchschnittliche Bodenbruttorente von $190 € /$ ha/a 
angesetzt. Die in Kapitel 2.5.3.2 ermittelten einmaligen Kosten der Planung, Ausweisung und Kontrolle finden in den laufenden Kosten von $4 € /$ Baum/a Berücksichtigung.

Im Ergebnis (s. Tabelle 15) liegt der Mittelwert des Kapitalwerts des Erhalts eines Habitatbaums über einen 30-jährigen Zeitraum, in dem der Baum durch Absterben, Pilzbefall, etc. vollständig seinen wirtschaftlichen Wert einbüßt im Schnitt bei $522 €$.

Tabelle 15: Kosten des Einzelbaumerhalts, Probefläche 1 Hämeler Wald Abt. 1 in €/Baum, Berechnung gem. Bewertungskonzept für Einzelbäume

\begin{tabular}{|c|c|c|c|c|c|c|c|c|c|c|c|c|c|}
\hline \multicolumn{14}{|c|}{ Kalkulationsvorgaben } \\
\hline \multicolumn{6}{|c|}{ Periodendauer in Jahren } & \multirow{3}{*}{\multicolumn{2}{|c|}{\begin{tabular}{r|}
30 \\
$1,5 \%$ \\
1
\end{tabular}}} & \multirow{2}{*}{\multicolumn{5}{|c|}{ laufende Kosten in EUR/Baum/a }} & 4,00 \\
\hline \multicolumn{6}{|c|}{ Kalkulationszins } & & & & & & & & \\
\hline \multicolumn{6}{|c|}{ Bodenbruttorente in EUR/ha/a } & & & & & & & & \\
\hline \multicolumn{5}{|c|}{ Baumdaten } & \multirow{2}{*}{ 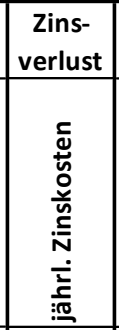 } & \multicolumn{2}{|c|}{ Wertminderung } & \multicolumn{3}{|c|}{ Ertragsverlust } & Sonstiges & \multicolumn{2}{|c|}{$\begin{array}{c}\text { Kosten je } \\
\text { Baum }\end{array}$} \\
\hline \multirow[t]{2}{*}{ zे } & \multirow[t]{2}{*}{ 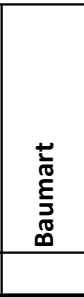 } & 号 & 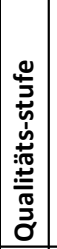 & 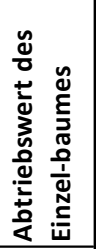 & & 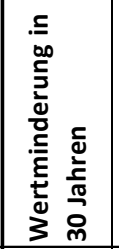 & 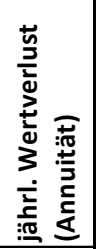 & 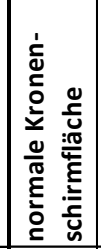 & 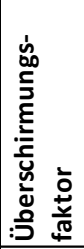 & 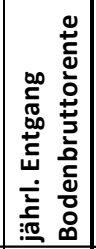 & 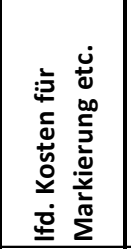 & 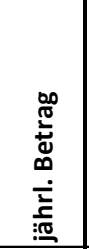 & $\begin{array}{l}\frac{1}{0} \\
\frac{3}{\pi} \\
\frac{3}{00} \\
\frac{0}{00} \\
\underline{0}\end{array}$ \\
\hline & & $\mathrm{cm}$ & & EUR & EUR/a & in $\%$ & EUR/a & $\mathrm{qm}$ & & EUR/a & EUR/a & EUR/a & EUR \\
\hline 1 & Buche & 73 & 4 & $240 €$ & $3,6 €$ & $-100 \%$ & $6,4 €$ & 133 & 1,4 & $3,5 €$ & $4,0 €$ & $18 €$ & $422 €$ \\
\hline 2 & Eiche & 82 & 4 & $448 €$ & $6,7 €$ & $-100 \%$ & $11,9 €$ & 115 & 1,5 & $3,3 €$ & $4,0 €$ & $26 €$ & $623 €$ \\
\hline \multicolumn{4}{|c|}{ Mittelwert: } & $344 €$ & $5,2 €$ & & $9 €$ & & & $3,4 €$ & $4,0 €$ & $22 €$ & $522 €$ \\
\hline
\end{tabular}

Das Fallbeispiel wurde auf Grundlage der FFH-Verordnung Niedersachsens (Niedersächsisches Ministerium für Umwelt, Energie und Klimaschutz 2015) berechnet, die für den vorliegenden Erhaltungszustand A eine Ausweisung von sechs Habitatbäumen je Hektar vorsieht. Somit belaufen sich die jährlichen Gesamtkosten des Erhalts je Hektar Lebensraumtypfläche im Erhaltungszustand $A$ in diesem Beispielfall auf $132 € /$ ha/a.

Durch Ablotung der tatsächlichen Kronenschirmflächen an den maximalen seitlichen Ausdehnungen der ausgewählten Habitatbäume konnte gezeigt werden, dass die erlassgemäß vorgeschriebenen sechs zu erhaltenden Habitatbäume je Hektar einen Anteil von 11 \% des Gesamtbestands überschirmen und mit zunehmendem Alter und Absterbeprozess unmittelbar durch herabfallende Äste gefährden. Dieser Gefährdungsbereich ist wesentlich größer als der Anteil der Bäume an der Stammzahl bzw. dem rechnerischen Kronenflächenanteil auf Grundlage des Waldwachstumsmodell von Nagel (2009). Dies ist auf zwei Gründe zurückzuführen. Erstens werden für die Ausweisung von ökologisch wertvollen Bäumen vornehmlich besonders alte oder große Exemplare ausgewählt, die aufgrund ihrer Struktur einen hohen ökologischen Wert durch Rindenabplatzungen, Mulmhöhlen, Horste, Totäste oder Spechthöhlen aufweisen. Je mehr dieser Einzelmerkmale ein Baum aufweist, desto höher ist sein ökologischer Nutzen. Das hohe Alter 
oder die besondere Struktur (bevorzugt werden auch durchgewachsene Mittelwaldrelikte ausgewählt) führen in der Praxis zu Kronenschirmflächen, die deutlich über den durchschnittlichen Werten des Bestandes liegen. Zweitens führen insbesondere bereits vorhandene und ökologisch wertvolle Totstarkäste zu einer unmittelbaren Gefährdung sich im Wald bewegender oder arbeitender Personen. Der Erhalt dieser Bäume bis hin zum biologischen Lebensende führt dabei kontinuierlich zu einer Zunahme der Totäste und somit der Gefährdung umliegender Bereiche. Dabei ist das vollständige Absterben des Baumes und der Übergang zu stehendem Totholz noch nicht berücksichtigt.

Wenn ein vollständiges Absterben einsetzt oder auch nur ein Absterben der Wurzeln erfolgt, steigt der Gefährdungsbereich des Habitatbaums auf einen Radius seiner Baumlänge wie Putensen (2018) es in ihrer Arbeit, dargestellt im nachfolgenden Kapitel 4.5, detailliert analysiert hat.

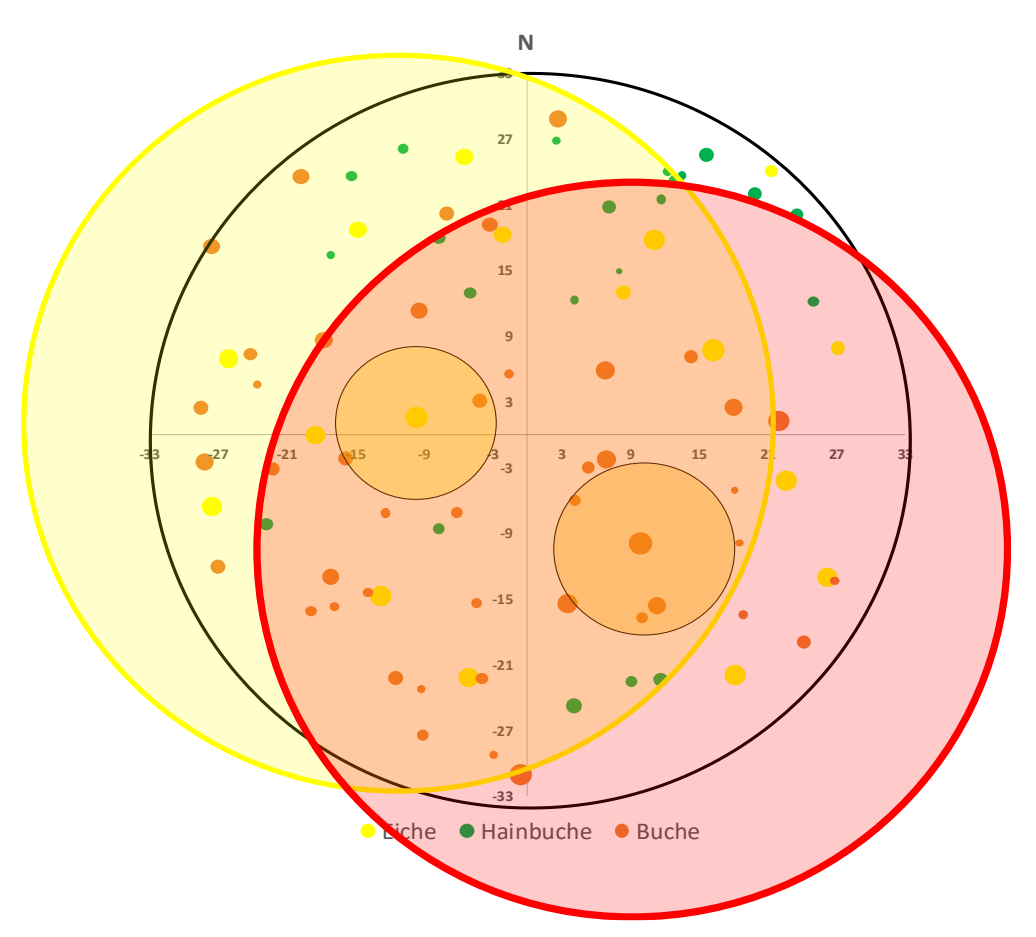

Abbildung 48: Stammverteilungsplan Probefläche 1 Hämeler Wald Abt. 1, Gefahrenbereiche der ausgewählten Habitatbäume als farbig-transparente Kreise, $r=$ Baumhöhe

Aus dem Erhalt der Alt- und Habitatbäume bei gleichmäßiger räumlicher Verteilung resultiert ein erhebliches, die gesamte Fläche betreffendes Risiko im Bereich des Arbeitsschutzes und der Verkehrssicherungspflicht für den Waldbesitzer. Nur wenige Programme oder Verträge berücksichtigen dieses bislang im angemessenen Umfang. Auch im Rahmen der Fallstudie konnte lediglich auf das Problem hingewiesen werden. Zur Lösung bedarf es neben weiterer Forschung, insbesondere vertraglicher Regelungen, in denen bspw. der Vertragspartner dieses Risiko übernimmt. 


\subsubsection{Baumartenwechsel}

Neben dem Erhalt von Habitatbäumen enthalten die zu erlassenden Schutzgebietsverordnungen auch Vorgaben zur Zusammensetzung künstlicher Verjüngung. So ist die ordnungsgemäße Forstwirtschaft von Verboten freigestellt, sofern:

1. In Flächen mit wertbestimmenden Lebensraumtypen im Erhaltungszustand A bei künstlicher Verjüngung lebensraumtypische Baumarten und auf mindestens 90 \% der Verjüngungsfläche lebensraumtypische Hauptbaumarten angepflanzt werden,

2. In Flächen mit wertbestimmenden Lebensraumtypen im Erhaltungszustand $B / C$ bei künstlicher Verjüngung

a. ausschließlich lebensraumtypische Baumarten und dabei auf mindestens $80 \%$ der Verjüngungsfläche lebensraumtypische Hauptbaumarten oder

b. auf mindestens $90 \%$ der Verjüngungsfläche lebensraumtypische Baumarten angepflanzt oder gesät werden.

Die Probefläche 2 im Hämeler Wald war ein reiner Kiefernbestand, der nach dem Schutzzweck des FFH-Gebiets keinen wertbestimmenden Lebensraumtyp darstellt, nach grundsätzlicher Planung des FFH-Gebiets jedoch naturschutzfachlich zu entwickeln ist. Bei der Bewertung wurde unterstellt, dass bei einer planmäßigen, nicht durch FFH beeinflussten Endnutzung und der Begründung einer Folgebestockung, der Waldbesitzer keinen Reinbestand mehr begründen würde, sondern, ökonomisch motiviert und zur Absenkung von Bestandesrisiken, neben der Kiefernnaturverjüngung eine künstliche Anreicherung mindestens einer weiteren Baumart (hier Douglasie) vornähme. Die ökologisch begründeten Schutzgebietsvorgaben schließen diese Baumarten in Lebensraumtypflächen jedoch aus, so dass eine vollständige Begründung mit Buche notwendig wäre.

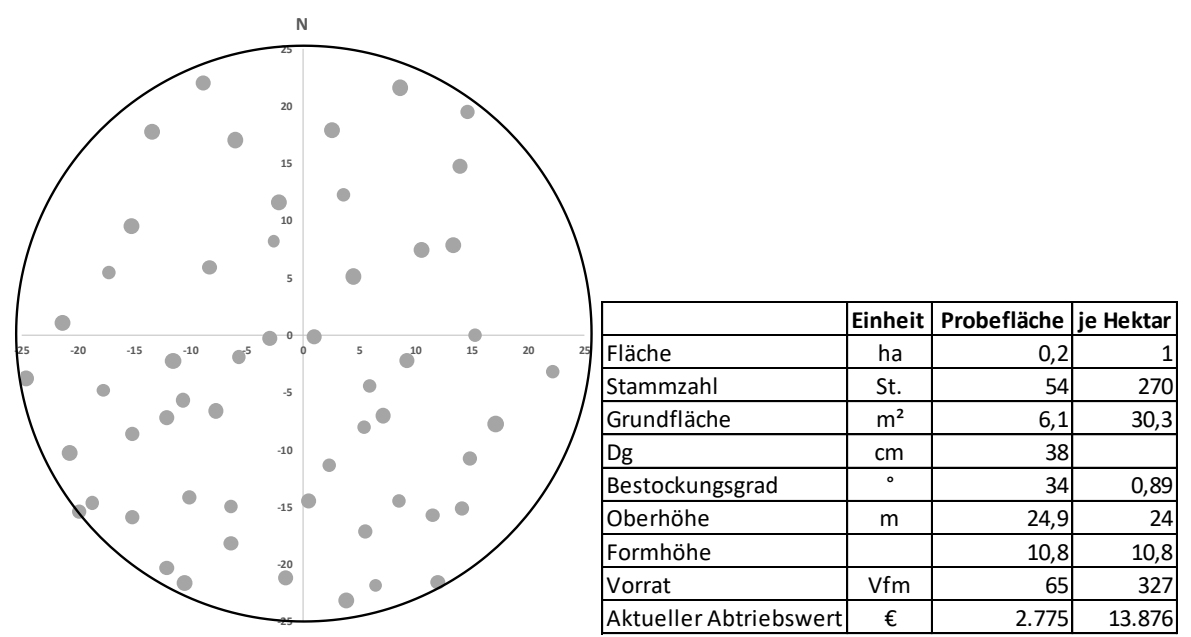

Abbildung 49: Stammverteilungsplan Probefläche 2 Hämeler Wald Abt. 2, Punktgröße in Abhängigkeit des Baumdurchmessers, Farbe in Abhängigkeit der Baumart 
Für den ökonomischen Referenzbestand wird eine Leistungsklasse (LKL) der Kiefer von 9, entsprechend des Vorbestands unterstellt, die Douglasie wird auf diesem Standort mit LKL 16 taxiert. Die Kiefer wird naturverjüngt, es fallen lediglich Kosten der Bestandesregulierung an, die Douglasie wird mit einem Flächenanteil von 30 \% im Weitverband gepflanzt.

In der ökologischen Alternative muss die gesamte Fläche mit Buche künstlich bestockt werden, wobei für einen solchen Waldumbau in Niedersachsen von einer 80-prozentigen Förderung ausgegangen wird. Die Leistungsklasse der Buche wird entsprechend den Beständen der Umgebung mit 8 angenommen.

Tabelle 16: Ertragsverlust durch Baumartenwechsel, Probefläche 2 Hämeler Wald Abt. 2, in €/ha/a, Berechnung gem. Bewertungskonzept für forstliche Nutzungsbeschränkungen

\begin{tabular}{|c|c|c|c|c|c|c|c|c|c|}
\hline & Baumart & $\begin{array}{c}\text { Umtriebszeit } \\
{[\mathrm{a}]}\end{array}$ & LkI & Wz & \begin{tabular}{|c|} 
Kosten \\
Verjüngung \\
{$[€ /$ ha] }
\end{tabular} & $\begin{array}{c}\text { Mischung } \\
{[\%]}\end{array}$ & $\begin{array}{c}\text { waldbaulicher } \\
\text { DB je BA } \\
{[€ / \mathrm{ha} / \mathrm{a}]} \\
\end{array}$ & $\begin{array}{c}\text { waldbaulicher } \\
\text { DB } \\
{[€ / \mathrm{ha} / \mathrm{a}]} \\
\end{array}$ & $\begin{array}{c}\text { Differenz } \\
\text { dauerhaft } \\
{[€ / \mathrm{ha} / \mathrm{a}]} \\
\end{array}$ \\
\hline \begin{tabular}{l|}
$\frac{c}{u}$ \\
$\frac{. m}{\varepsilon}$
\end{tabular} & Kiefer & 100 & 9 & 3 & 660 & $70 \%$ & 110 & \multirow{2}{*}{204} & \multirow{3}{*}{-130} \\
\hline $\begin{array}{l}\text { 气े } \\
\text { : }\end{array}$ & Douglasie & 70 & 16 & 3 & 3.100 & $30 \%$ & 423 & & \\
\hline 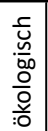 & Buche & 140 & 8 & 3 & $1.360^{*}$ & $100 \%$ & 74 & 74 & \\
\hline
\end{tabular}

Die ökonomische Referenz lässt einen jährlichen Holzproduktionswert (waldbaulicher Deckungsbeitrag) von $204 € /$ ha/a in der Mischung von Kiefer und Douglasie erwarten. Demgegenüber steht der waldbauliche Deckungsbeitrag von $74 € / h a / a^{41}$ in der ökologischen Alternative. Daraus resultiert eine dauerhafte Ertragsdifferenz von -130 €/ha/a durch die Einschränkung der Baumartenwahl in Folge der Auflagen der Naturschutzgebietsverordnung.

\subsubsection{Umtriebszeitverlängerung}

Gemäß dem Erlass für die aufzustellenden Naturschutzgebietsverordnungen müssen auf Waldflächen mit wertbestimmenden Lebensraumtypen im Erhaltungszustand A Altholzanteile (BHD >50 cm, bzw. 100 Jahre und älter) von mindestens $35 \%$ der Lebensraumtypfläche des jeweiligen Eigentümers erhalten bleiben. Für den Erhaltungszustand $B / C$ gelten entsprechend $20 \%$ Altholzanteile der Lebensraumtypfläche.

In der Waldbewirtschaftung bedeutet dies eine Verlängerung der Produktionsdauer über die optimale Umtriebszeit hinaus, um den Flächenanteil der Altholzbestände auf dem gewünschten Niveau zu erhalten.

\footnotetext{
${ }^{41}$ Wegen der spezifischen örtlichen Berechnung unter Berücksichtigung der Bestandesbegründungskosten korrespondiert das Ergebnis nicht unmittelbar mit den in Tabelle 8 dargestellten Werten.
} 
Die Probefläche 3 war ein reiner Buchenaltholzbestand mit einem Durchmesser des Grundflächenmittelstamms (Dg) von $49 \mathrm{~cm}$. Aus klassischer forstlicher Behandlungssicht sind die vorherrschenden Bäume hiebsreif und die Endnutzung könnte eingeleitet werden. Unter Berücksichtigung der Auflagen wäre der Bestand noch 20 Jahre länger zu erhalten.

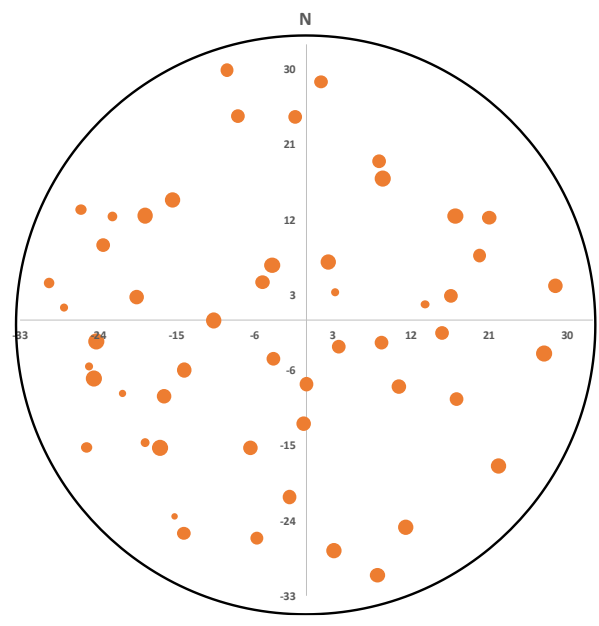

\begin{tabular}{|l|c|r|r|}
\hline & Einheit & Probekreis & je Hektar \\
\hline Fläche & ha & 0,33 & 1 \\
\hline Stammzahl & St. & 52 & 156 \\
\hline Grundfläche & $\mathrm{m}^{2}$ & 9,7 & 29,2 \\
\hline Dg & $\mathrm{cm}$ & 49 & \\
\hline Bestockungsgrad & ${ }^{\circ}$ & 0,88 & 0,88 \\
\hline Oberhöhe & $\mathrm{m}$ & 34 & 33,1 \\
\hline Formhöhe & & 17,2 & 17,2 \\
\hline Vorrat & $\mathrm{Vfm}$ & 168 & 503 \\
\hline Abtriebswert & $€$ & 7.743 & 23.231 \\
\hline
\end{tabular}

Abbildung 50: Stammverteilungsplan Probefläche 3 Hämeler Wald Abt. 3, Punktgröße in Abhängigkeit des Baumdurchmessers, Farbe in Abhängigkeit der Baumart

Als ökonomische Referenzbewirtschaftung wurde hier die Endnutzung im Alter 140 Jahre angenommen. Daraus ergibt sich (äquivalent zur ökologischen Alternative der Probefläche 2) ein jährlicher Holzproduktionswert von $74 € /$ ha/a. Die ökologische Alternative ist der Erhalt um weitere 20 Jahre bis zum Alter 160, um den dauerhaften Bestand an Altbeständen zu sichern. In dieser Zeit setzt eine wirtschaftliche Entwertung des Holzes durch zunehmende Verkernung und das Absinken der Zuwächse ein. Der waldbauliche Deckungsbeitrag beträgt in diesem Zeitraum $-276 € /$ ha/a ${ }^{42}$. Daraus resultiert eine Differenz, bzw. Kosten des Erhalts von $-350 € /$ ha/a in entsprechenden Altholzbeständen.

Tabelle 17: Ertragsverlust durch Verlängerung der Umtriebszeit, Probefläche 3 Hämeler Wald Abt. 3, in €/ha/a

\begin{tabular}{|l|c|c|c|c|c|c|c|}
\cline { 2 - 7 } \multicolumn{1}{c|}{} & Baumart & $\begin{array}{c}\text { Umtriebszeit } \\
{[\mathrm{a}]}\end{array}$ & LkI & Wertziffer & $\begin{array}{c}\text { Mischungsanteil } \\
{[\%]}\end{array}$ & $\begin{array}{c}\text { waldbaulicher } \\
\text { DB } \\
{[€ / \mathrm{ha} / \mathrm{a}]}\end{array}$ & $\begin{array}{c}\text { Ertragsverlust } \\
\text { 20 Jahre } \\
{[€ / \text { ha/a] }}\end{array}$ \\
\hline ökonomisch & Buche & 140 & 8 & 3 & $100 \%$ & 74 & \multirow{2}{*}{350} \\
\hline ökologisch & Buche & $\begin{array}{c}\text { Verlängerung } \\
140-160\end{array}$ & 8 & $\begin{array}{c}\text { Abfall von } \\
3-5\end{array}$ & $100 \%$ & -276 & \\
\hline
\end{tabular}

\footnotetext{
${ }^{42}$ Der negative Betrag resultiert aus dem Wertabfall des stehenden Holzes sowie den Kosten der Verhinderung des Folgebestands.
} 


\subsubsection{Zusammenfassung}

Die naturschutzfachlichen Auflagen für Flächen mit wertbestimmenden Lebensraumtypen gem. der niedersächsischen FFH-Verordnung (Niedersächsisches Ministerium für Umwelt, Energie und Klimaschutz 2015) sind vielfältig. Die drei in den vorigen Abschnitten im Rahmen der Fallstudie bewerteten Auflagen stellen sicherlich den wirtschaftlich stärksten Eingriff in die bisherige Bewirtschaftung des Waldes dar. Die drei Auflagen (Habitatbaumerhalt, Baumartenwechsel und Umtriebszeitverlängerung/Verzögerung der Endnutzung) werden in unterschiedlicher Intensität in betroffenen Betrieben wirksam. Während Habitatbäume auf der gesamten Fläche mit wertbestimmenden Lebensraumtypen ausgewiesen werden müssen, ist die Erhöhung der Umtriebszeit in Abhängigkeit des Erhaltungszustands nur partiell notwendig. Bei einem Altholzanteil von $20 \%$ in Lebensraumtypen mit dem Erhaltungszustand $B / C$ ist dieser durch die reguläre Umtriebszeit von bspw. 140 Jahren bei der Buche im Rahmen einer gleichmäßigen Altersklassenverteilung automatisch vorhanden. Im Erhaltungszustand A mit einer geforderten Fläche von 35 \% müssen jedoch 10 \% Altholzbestände zusätzlich der Verzögerung zugeführt werden.

Der Baumartenwechsel in der künstlichen Verjüngung, insbesondere der Verzicht auf den weiteren Anbau von Nadelhölzern wirkt sich je nach Erhaltungszustand ebenfalls unterschiedlich aus. Im Erhaltungszustand A muss vollständig auf die hier als ökonomische Referenz unterstellte 30-prozentige Beimischung verzichtet werden, wohingegen im Erhaltungszustand B/C noch 10 \% Nadelhölzer zulässig sind. Die Belastung betrifft somit 20 \% der Fläche (s. Tabelle 18).

Tabelle 18: Zusammenfassung Auswirkungen naturschutzfachlicher Auflagen in der Fallstudie Hämeler Wald, in $€ / h a / a$

\begin{tabular}{|c|c|c|c|c|c|c|}
\hline \multirow{3}{*}{$\begin{array}{l}\text { Berechnung durchschnittlicher } \\
\text { Belastungen für Lebensraumtypen }\end{array}$} & \multicolumn{3}{|c|}{ Erhaltungszustand $\mathrm{A}$} & \multicolumn{3}{|c|}{ Erhaltungszustand $\mathrm{B} / \mathrm{C}$} \\
\hline & \multirow{2}{*}{$\begin{array}{c}\text { Anteil } \\
\text { Maßnahmen- } \\
\text { fläche [\%] }\end{array}$} & \multicolumn{2}{|c|}{ Ifd. jährl. Ertragsverlust [€/Jahr] } & \multirow{2}{*}{\begin{tabular}{|c|} 
Anteil \\
Maßnahmen- \\
fläche [\%]
\end{tabular}} & \multicolumn{2}{|c|}{ Ifd. jährl. Ertragsverlust [€/Jahr] } \\
\hline & & $\begin{array}{c}\text { pro ha } \\
\text { Maßnahmenfläche }\end{array}$ & $\begin{array}{c}\text { pro ha } \\
\text { Betriebsfläche }\end{array}$ & & $\begin{array}{c}\text { pro ha } \\
\text { Maßnahmenfläche }\end{array}$ & $\begin{array}{c}\text { pro ha } \\
\text { Betriebsfläche }\end{array}$ \\
\hline Habitatbaumerhalt [6(3) Bäume/ha] & 100 & 132 & 132 & 100 & 66 & 66 \\
\hline Verzögerung der Endnutzung & 10 & 350 & 35 & 0 & 350 & 0 \\
\hline Baumartenwechsel & 30 & 130 & 39 & 20 & 130 & 26 \\
\hline Gesamt & & & 206 & & & 92 \\
\hline
\end{tabular}

Die wirtschaftlichen Erschwernisse belaufen sich in der durchgeführten Fallstudie gewichtet mit den Maßnahmenflächenanteilen auf $206 € /$ ha/a in Lebensraumtypflächen im Erhaltungszustand $A$ und auf $92 € /$ ha/a in Lebensraumtypflächen im Erhaltungszustand B/C.

Nicht monetär bewertet, aber aufgrund der vollständigen Flächenbetroffenheit sehr schwer wiegt zusätzlich das steigende Arbeitssicherheits- und Verkehrssicherungsrisiko durch absterbende Habitatbäume. Die sonstigen Auflagen (40 m-Rückegassenabstand in Altholzbeständen, Herbizidverbot, Einschränkung der Nutzungszeit, etc.) müssen im Einzelfall gesondert bewertet werden. 


\subsection{Auswirkungen verschiedener Verteilungskonzepte von Habitatbäu- men}

Alt- \& Habitatbäumen wird eine hohe Bedeutung zur Erhaltung der Biodiversität im Wald beigemessen. Jedoch zeigt sich, dass der Erhalt von ökologisch wertvollen Bäumen, neben dem reinen ökonomischen Verlust durch Nichtnutzung auch zu weiteren betrieblichen Herausforderungen führt (vgl. Kapitel 0). Ein in der Theorie und den vorhandenen Praxishilfen bislang nur in Ansätzen berücksichtigter Aspekt ist die Wahl der räumlichen Verteilung der ausgewählten Bäume. In einer im Rahmen der vorliegenden Arbeit betreuten Masterarbeit (Putensen 2018) wurden fünf Konzepte zur Baumauswahl erstellt und als Fallstudie hinsichtlich verschiedener Auswahl- und Verteilungsaspekte untersucht.

\subsubsection{Ziel}

In der Arbeit wurde neben der ökonomischen Bewertung der ausgewählten Bäume auch den Folgen der räumlichen Verteilung nachgegangen. Die zentralen Fragestellungen waren:

1. Wie hoch ist die ökonomische Belastung der Waldbesitzer durch Habitatbaumausweisung?

2. Welche Vor- und Nachteile resultieren aus den gewählten Konzepten?

3. Welchen Einfluss hat das gewählte räumliche Verteilungskonzept der Bäume?

4. Welche Konzepte und welches Vertragsentgelt sind empfehlenswert?

\subsubsection{Methodik}

In sieben Laubholzbeständen (Buchen- und Buchen-Eichen-Mischbeständen, Alter: >120 Jahre) im Hochsauerlandkreis, Nordrhein-Westfalen, wurden im Oktober 2017 Bäume mit Habitatmerkmalen identifiziert und folgende Daten aufgenommen: Baumhöhe, BHD, Qualitätsstufe, Koordinaten und Habitatmerkmale. Diese Bäume eignen sich aufgrund einzelner oder mehrerer Merkmale für die mögliche Verwendung als Habitatbaum, da sie einen wichtigen ökologischen Zweck erfüllen. Nachfolgend werden diese Bäume als Habitatbaumanwärter bezeichnet. 


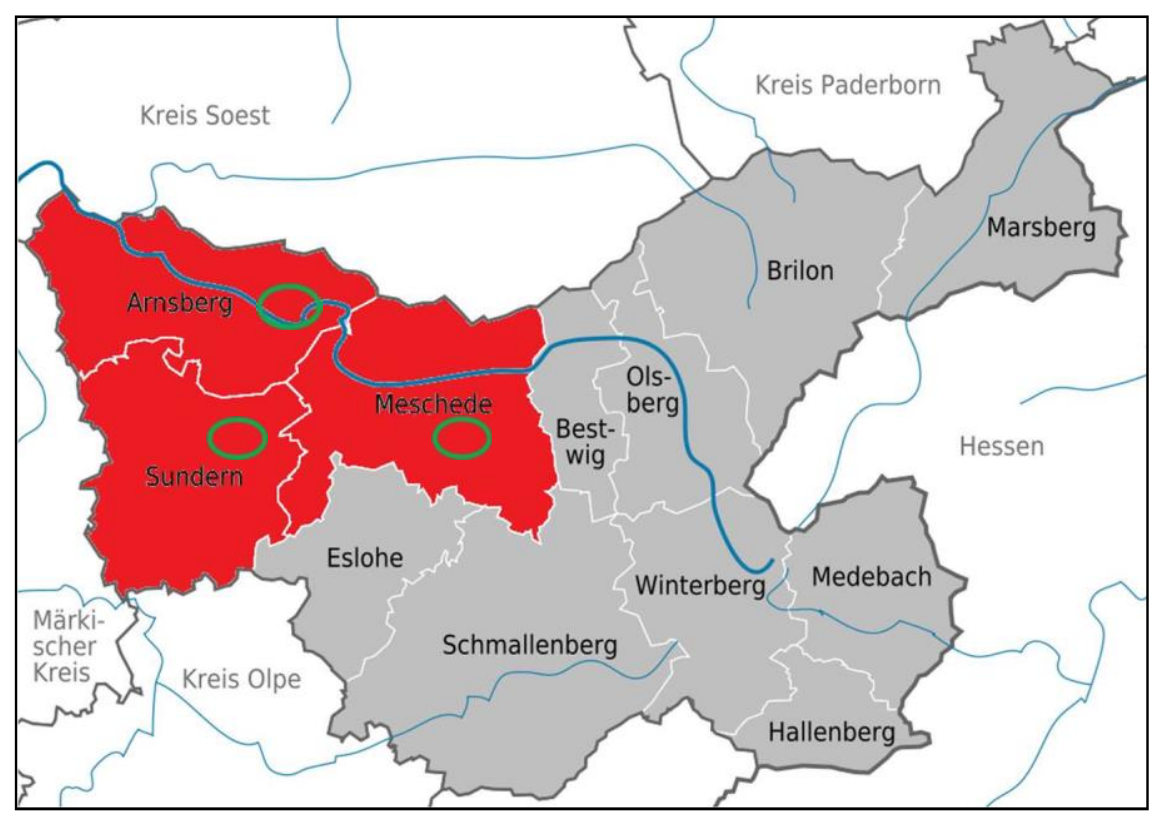

Abbildung 51: Lage der untersuchten Flächen im Hochsauerlandkreis. Grün: Lage der untersuchten Flächen, rot: Gebiete der Landschaftspläne. Quelle: Putensen 2018

Mit Hilfe der erfassten Daten wurde jeder Habitatbaumanwärter ökologisch in Anlehnung an Meier (2009)und ökonomisch nach Möhring (2010) bewertet. Bei der ökologischen Bewertung wurden für die vorhandenen Habitatmerkmale, den BHD und die Baumart ökologische Punktwerte im Anhalt an Meier (2009) vergeben und für jeden Baum aufsummiert.

Mittels dieses Punktwertansatzes wurden den ausgewählten Habitatbaumanwärtern ökologische Wertpunkte zwischen 3 bis 46,75 Punkte zugewiesen. Im arithmetischen Mittel lag der ökologische Wert bei 22,52 Punkten, bei einer Standardabweichung von 9,12 Punkten (Putensen 2018). Dabei konnte weder für die Baumart Buche noch für die Eiche ein Zusammenhang zwischen BHD und ökologischer Wertigkeit festgestellt werden (s. Abbildung 52).

Die ökonomische Bewertung erfolgte für einen Bewertungszeitraum von 20 Jahren. Für den Vergleich zwischen Konzepten ist der absolute Bewertungszeitraum zweitrangig, solange er für alle Konzepte identisch ist. Auf Grundlage des BHD und der Höhe wurde das Stammvolumen berechnet und aufbauend auf der Waldbewertungsrichtlinie NRW 2013 (Stand 2015) der Abtriebswert ermittelt. Die Opportunitätskosten der Kapitalbindung wurden mit einem Kalkulationszins von 1,5\% berechnet. Außerdem wurde ein Wertverlust von $25 \%$ in 20 Jahren unterstellt. Der Ertragsverlust der überschirmten Fläche, die aus dem BHD abgeleitet wurde, wurde mit dem Bewertungskonzept von Möhring et al. (2017) ermittelt. Neben diesen Verlusten wurde noch ein einmaliger Aufwand für die Auswahl und Markierung von $15 € /$ Baum unterstellt und laufende jährliche Erschwernisse von $6 € /$ Baum durch die Berücksichtigung bei Planungen, Hiebsmaßnahmen etc. angenommen (vgl. Möhring 2010). 


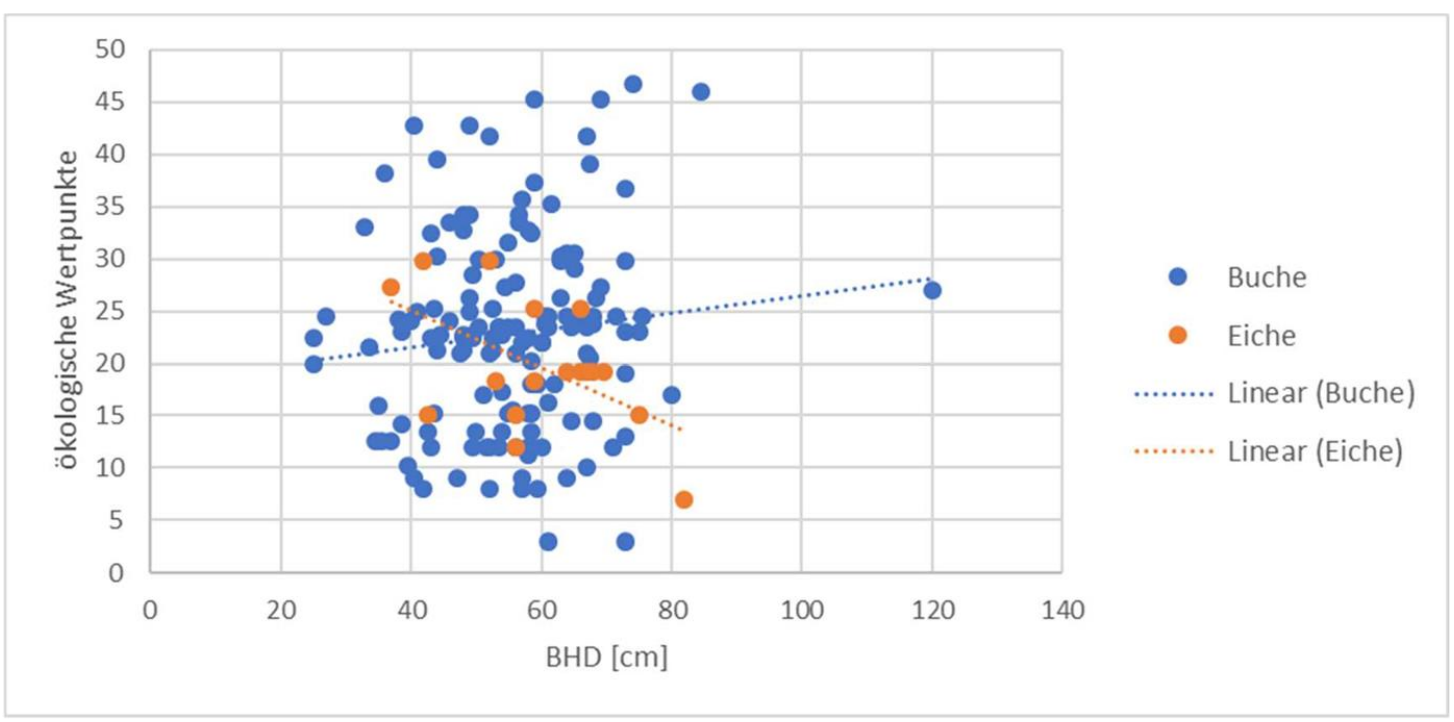

Abbildung 52: Zusammenhang zwischen BHD und Summe der ökologischen Wertpunkte pro Baum, Quelle: Putensen 2018

\subsubsection{Ergebnisse}

Die ökologische Bewertung der Habitatbäume in den Beständen ergab auf Grundlage der Tabelle von Meier (2009) Werte von 3 bis 47 Punkte. Die ökonomische Bewertung ergab Verluste (bzw. Kosten) von $129 € /$ Baum bis $604 € /$ Baum für die ersten 20 Jahre des Erhalts. Zwischen den ökologischen und ökonomischen Bewertungen des Einzelbaums konnte kein Zusammenhang nachgewiesen werden (s. Abbildung 53).

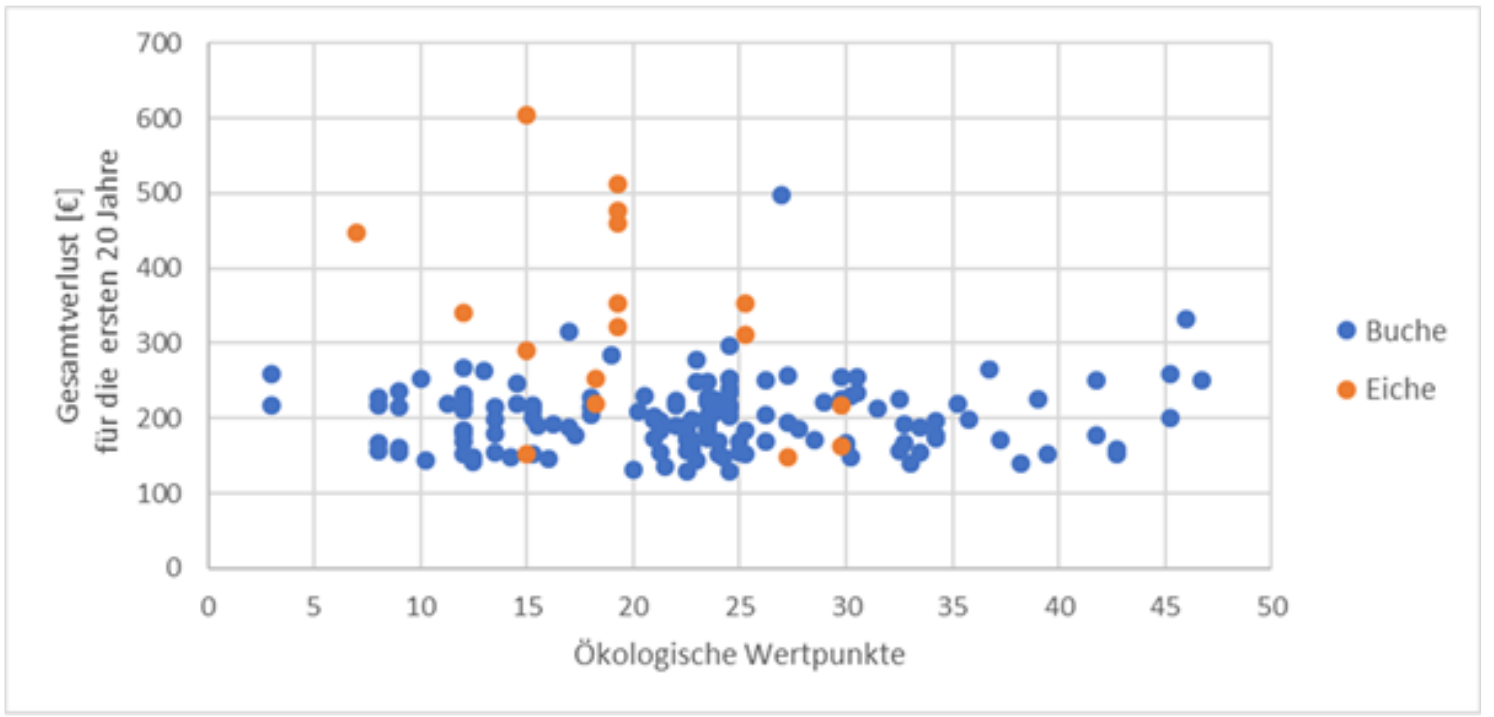

Abbildung 53: Verhältnis von ökologischen Wertpunkten und Gesamtverlust der Einzelbäume, $n=161$, Quelle: Putensen 2018

Nach der ökologischen und ökonomischen Einzelbaumbewertung der "Habitatbaumanwärter“ wurden für sieben Untersuchungsflächen jeweils fünf verschiedene Konzepte der Habitat- 
baumauswahl mit rund sechs Bäumen pro Hektar erstellt, bewertet und verglichen. Drei qualitative Konzepte orientierten sich an den ökologischen oder ökonomischen Rangfolgen der Bewertungen der Einzelbäume 1) nach den höchsten ökologischen Wertpunkten, 2) nach den geringsten ökonomischen Verlusten und 3) nach den geringsten resultierenden Quotienten (geringster ökonomischer Verlust im Verhältnis zum ökologischen Punktwert).

Bei den zwei räumlichen Konzepten wurden die Bäume 1) mit möglichst geklumpter und 2) mit möglichst gleichmäßiger Verteilung ausgewählt (s. Tabelle 19). Dazu wurde die Software R (Version 3.4.2) verwendet.

Tabelle 19: Entscheidungskriterien der fünf Konzepte, Quelle: Putensen 2018

\begin{tabular}{|c|c|c|c|c|c|}
\hline Konzept & Ökologisch & Ökonomisch & Quotient & Klumpung & Gleichmäßig \\
\hline Kriterium & $\begin{array}{l}\text { Ökologischer } \\
\text { Wert der } \\
\text { Einzelbäume }\end{array}$ & $\begin{array}{l}\text { Ökonomischer } \\
\text { Verlust bei } \\
\text { Ausweisung } \\
\text { der } \\
\text { Einzel bäume } \\
\text { für } 20 \text { Jahre }\end{array}$ & $\begin{array}{l}\text { Ökonomischer } \\
\text { Verlust (für } 20 \\
\text { Jahre) pro } \\
\text { Ökowertpunkt } \\
\text { der } \\
\text { Einzelbäume } \\
\text { (€/Punkt) }\end{array}$ & $\begin{array}{l}\text { Maximaler } \\
\text { Baumabstand } \\
\text { jeder möglichen } \\
\text { Baumkombination } \\
\text { mit } 6 \text { Bäumen pro } \\
\text { ha }\end{array}$ & $\begin{array}{l}\text { Minimaler } \\
\text { Baumabstand } \\
\text { jeder möglichen } \\
\text { Baumkombination } \\
\text { mit } 6 \text { Bäumen pro } \\
\text { ha }\end{array}$ \\
\hline Auswahl & $\begin{array}{l}\text { Die Bäume mit } \\
\text { den höchsten } \\
\text { ökologischen } \\
\text { Werten }\end{array}$ & $\begin{array}{l}\text { Die Bäume mit } \\
\text { den geringsten } \\
\text { ökonomischen } \\
\text { Verlusten }\end{array}$ & $\begin{array}{l}\text { Die Bäume mit } \\
\text { den kleinsten } \\
\text { Quotienten } \\
\text { (geringste } \\
\text { Kosten pro } \\
\text { Punkt) }\end{array}$ & $\begin{array}{l}\text { Die Kombination } \\
\text { mit dem kleinsten } \\
\text { Maximal-Abstand }\end{array}$ & $\begin{array}{l}\text { Die Kombination } \\
\text { mit dem größten } \\
\text { Minimal-Abstand }\end{array}$ \\
\hline $\begin{array}{l}\text { Ggf. Zweites } \\
\text { Kriterium }\end{array}$ & & & & \multicolumn{2}{|c|}{$\begin{array}{l}\text { Mittlerer Baumabstand jeder } \\
\text { Kombination }\end{array}$} \\
\hline Auswahl & & & & $\begin{array}{l}\text { Die Kombination } \\
\text { mit dem kleinsten } \\
\text { mittleren } \\
\text { Baumabstand }\end{array}$ & $\begin{array}{l}\text { Die Kombination } \\
\text { mit dem größten } \\
\text { mittleren } \\
\text { Baumabstand }\end{array}$ \\
\hline
\end{tabular}

Die Bewertung der fünf gewählten Auswahlkonzepte erfolgte anhand der Bewertungskriterien ökologische Punktwerte, ökonomischer Gesamtverlust, Verlust pro ökologischem Punktwert, Kronenschirmfläche (Flächeninanspruchnahme) und potenzieller Gefahrenbereich bei einer ${ }^{43}$ sowie doppelter ${ }^{44}$ Baumlänge (s. Abbildung 54 ).

\footnotetext{
${ }^{43}$ Eine Baumlänge entspricht dem Abstand zu öffentlichen Straßen im Rahmen der Verkehrssicherung ${ }^{44}$ Die doppelte Baumlänge soll bei manuellem Holzeinschlag zu Totholzbäumen eingehalten werden It. Berufsgenossenschaft SVLFG (2018).
} 


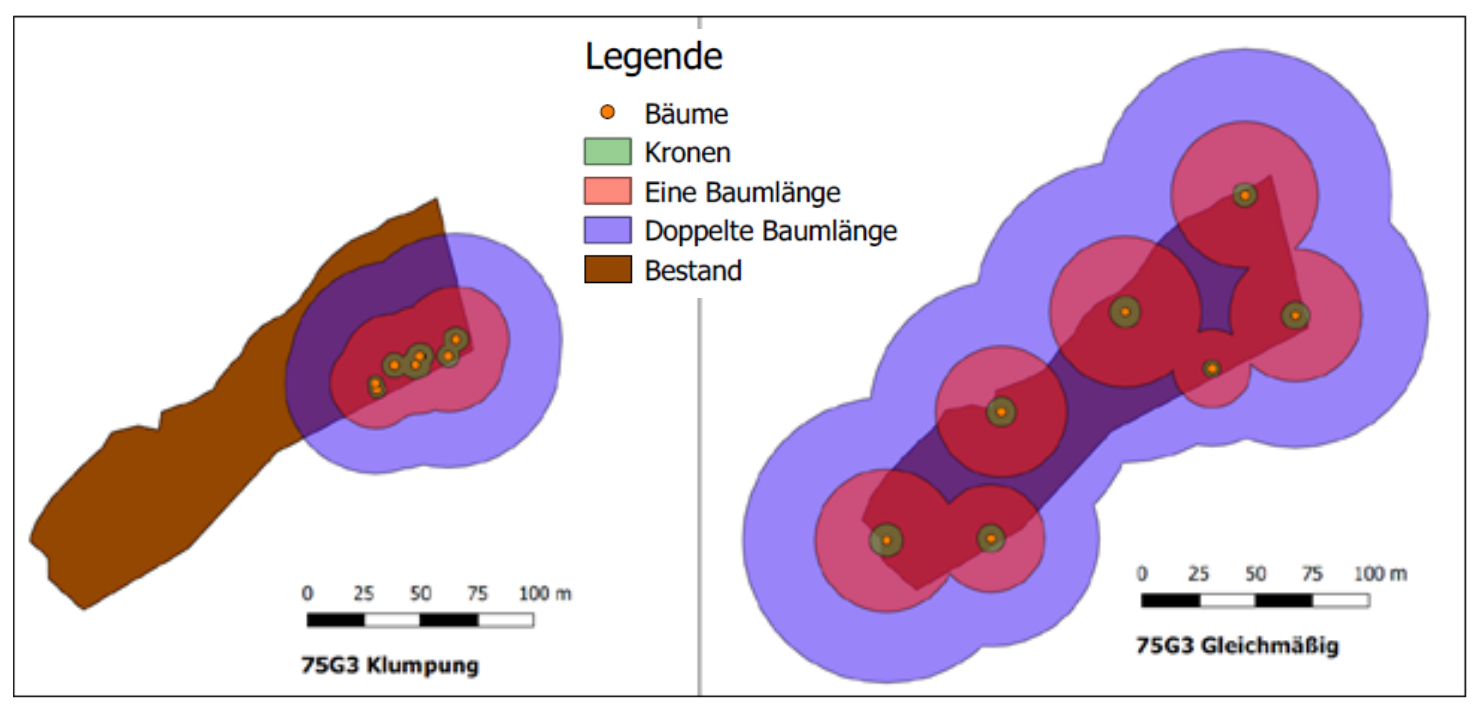

Abbildung 54: Veranschaulichung der potenziellen Gefährdungsbereiche an einer Beispielfläche. Szenario Klumpung: Bereich einer Baumlänge (rot): 29 \%, doppelter Baumlänge (lila): 84 \% der Bestandesfläche. Szenario Gleichmäßig: Bereich einer Baumlänge (rot): 144 \%, doppelter Baumlänge (lila): 368 \%, Quelle: Putensen 2018

Die qualitativ erstellten Konzepte (Auswahl der Bäume nach ökonomischen oder ökologischen Wertpunkten, bzw. nach Quotienten) sind auf jeder Fläche hinsichtlich ihres spezifischen Erstellungskriteriums vorteilhaft gegenüber den räumlichen Auswahlkonzepten. Das Quotienten-Konzept als Verschneidung von Ökologie und Ökonomie liegt hinsichtlich der ökologischen Wertigkeit nicht weit von der rein ökologischen Auswahl entfernt. Aus diesem Grund und wegen seiner höheren Effektivität und Effizienz gegenüber den anderen zwei qualitativen Konzepten wird dieses Konzept zur Habitatbaumauswahl empfohlen, wobei die tatsächliche Entscheidung immer von den jeweiligen Rahmenbedingungen abhängt. Die höhere Effektivität bei der Auswahl wird erkennbar, wenn Lorenzkurven für alle aufgenommenen Bäume ( $n=161)$ nach den Kriterien ökologische Punkte und ökonomische Verluste erstellt werden (Abbildung 55).
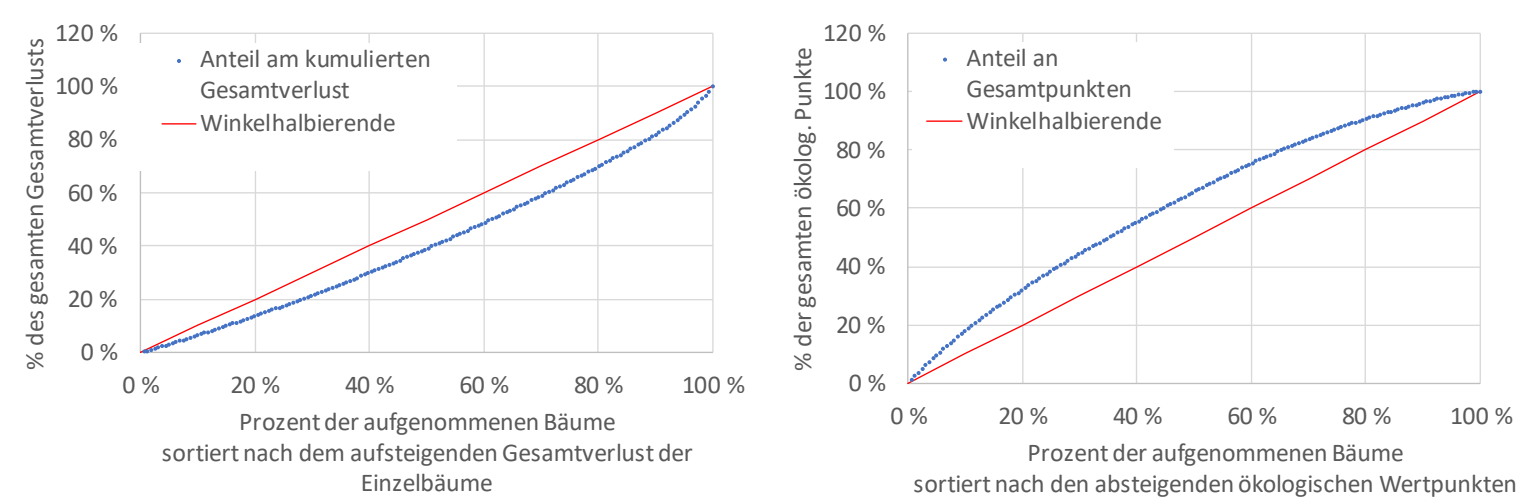

Abbildung 55: Lorenzkurven des relativen Anteils am Gesamtverlust bei Sortierung der 161 aufgenommenen Bäume nach dem ökonomischen Wert und den ökologischen Punkten. Quelle: Putensen 2018 
Je stärker eine Lorenzkurve (blau) von der Winkelhalbierenden (rot) abweicht, desto größer ist die Effektivität der Auswahl nach dem jeweiligen Kriterium. Das ökonomische Auswahlkonzept verursacht die geringeren Verluste, lässt jedoch die ökologische Wertigkeit der Bäume außer Acht und erzielt eine „Habitatbaumauswahl“ mit nur zufälligen, mittleren ökologischen Punkten, was die Zielgerechtigkeit der Ausweisung als Naturschutzmaßnahme fraglich erscheinen lässt.

Einige der untersuchten Flächen sind wegen geringer Anzahlen von Bäumen mit Habitatstrukturen bzw. geringer ökologischer Punktwerte der Bäume oder angrenzenden öffentlichen Wegen weniger geeignet für die Habitatbaumauswahl als andere Flächen. Die auf den jeweiligen Flächen untersuchten qualitativen und räumlichen Konzepte verursachen Gesamtverluste von $890 € /$ ha bis $1.187 € /$ ha für die ersten 20 Jahre, was fünf bis zehn Euro je ökologischem Wertpunkt für die ersten 20 Jahre entspricht. In diesen Summen nicht enthalten sind monetäre Anreize, die den Waldbesitzer zur Teilnahme motivieren könnten. Außerdem sind diese Kosten nicht pauschal auf andere Flächen anwendbar, da es sich um eine räumlich explizite, nicht repräsentative Fallstudie handelte.

Die obige Empfehlung für das Quotientenkonzept, also die vorrangige Auswahl der Bäume mit den geringsten ökonomischen Verlusten pro ökologischem Wertpunkt, ergeht ohne Beachtung der räumlichen Verteilung und des daraus resultierenden potenziellen Gefahrenbereichs. In diesem Bereich wird die Durchführung forstlicher Arbeiten wegen der aus dem zunehmenden Zerfall einsetzenden Gefährdung der Arbeitssicherheit erschwert, beispielsweise durch herabfallende Totäste, insbesondere während Fällungen benachbarter Bäume. Daher ist entweder eine angemessene, aber schwer im Voraus ermittelbare Entschädigung für diese betriebliche Einschränkung erforderlich oder es muss auf eine einzelstammweise Verteilung von Habitatbäumen verzichtet werden. Stattdessen sind geklumpte Habitatbaumausweisungen in Form von Bauminseln oder kleinen Flächenstilllegungen zu empfehlen. Abbildung 54 zeigt, dass die räumliche Verteilung der Bäume den potenziellen Gefahrenbereich erheblich beeinflusst: Der Gefahrenbereich einfacher Baumlänge ist beim gleichmäßigen Szenario fast fünfmal so groß wie beim geklumpten Szenario. Für den Bereich doppelter Baumlänge liegt der Unterschied etwas über dem Faktor vier. Die Abbildung 54 zeigt auch, dass nicht nur die Maßnahmenfläche von der Gefährdung betroffen ist, sondern unter Umständen auch große Teile von Nachbarflächen, was bei kleinflächigem Waldeigentum zu Konflikten führen kann. Außerdem sollten zur Berücksichtigung der Verkehrssicherungspflicht in Randbereichen von Flächen an öffentlich gewidmeten Straßen und Wegen keine Habitatbäume ausgewiesen werden. Diese Hinweise finden sich vermehrt auch in den Merkblättern und Ausweisungskonzepten der jeweiligen Bundesländer, die den Erhalt von Alt- und Habitatbäumen fördern (vgl. Bayrisches Staatsministerium für 
Ernährung, Landwirtschaft und Forsten 2014; Landesbetrieb ForstBW 2016). Nebenbei ist die Ausweisung von Habitatbäumen in Gruppen oder Inseln auch aus ökologischer Sicht vorteilhaft (Elmer et al. 2018).

\subsubsection{Zusammenfassung}

Im Rahmen der Masterarbeit konnte anhand einer Fallbeispielsanalyse gezeigt werden, dass das gewählte Konzept der Auswahl von Habitatbäumen großen Einfluss auf ökologische, ökonomische und betriebliche Auswirkungen hat. Im Sinne der Allokation von Ressourcen mit dem Ziel ökologisch und ökonomisch akzeptabler Ergebnisse ist das Quotientenkonzept zu empfehlen. Unbeachtet bleiben dabei die räumliche Verteilung und die einhergehenden Gefährdungsbereiche. Unter Berücksichtigung der Arbeitssicherheit bei der weiteren Bewirtschaftung der umliegenden Bereiche und der Verkehrssicherung sind klar Konzepte zu erwägen, die eine Konzentration der Risiken auf einer möglichst geringen Teilfläche bewirken. 


\section{Diskussion}

Die durchgeführten Befragungen der Fallbeispielsbetriebe im WaVerNa-Projekt und der BBForstbetriebe zielten neben der Status quo-Analyse darauf ab, die Schlüsselfaktoren für einen erfolgreichen Vertragsnaturschutz im Wald zu analysieren und auf Grundlage dieser „Eckpunkte“ die bisherigen Hemmnisse um Lösungsansätze unter besonderer Berücksichtigung der Erkenntnisse aus der Landwirtschaft zu erweitern, um Verbesserungsmöglichkeiten für den Vertragsnaturschutz im Wald aufzuzeigen. Die Unterschiedlichkeit der verfolgten Ziele, der verwendeten Methoden und die bisherigen Ergebnisse erfordern aus Sicht des Autors eine zweigeteilte Diskussion. Zur besseren Nachvollziehbarkeit erfolgt zunächst die Auseinandersetzung mit den Ergebnissen der Befragungen. Die Diskussion der Bewertungskonzepte für Nutzungseinschränkungen erfolgt im Anschluss.

\subsection{Diskussion der Befragung von Forstbetrieben}

Der Vertragsnaturschutz im Wald ist ein Instrument, das im Kontext der aktuellen Diskussionen um die Bereitstellung von Natur- und Klimaschutzleistungen des Waldes einen hohen Stellenwert besitzen könnte, allerdings ein Nischendasein fristet (Wissenschaftlicher Beirat für Waldpolitik und Wissenschaftlicher Beirat für Biodiversität und Genetische Ressourcen 2020, S. 4). Dieser Befund kann durch die dargestellten Ergebnisse der Befragung nur unterstrichen werden, da zwar eine Vielzahl von Betrieben im Ansatz Vertragsnaturschutzmaßnahmen umgesetzt haben, aber bspw. der Anteil am Betriebsertrag (s. 3.3.7) so gering ist, dass nicht von einer maßgeblichen Rolle im wirtschaftlichen Handeln ausgegangen werden kann.

Die befragten Forstbetriebe zählen zur Gruppe der privaten Erwerbsforstbetriebe, die die regelmäßige Holznutzung als Grundlage des wirtschaftlichen Erfolgs zur Hauptaufgabe haben. Damit unterscheiden sie sich vom Kleinprivatwald, dessen Ziele substantiell abweichen können und nach aktuellen Befragungen eher den Natur- und Klimaschutz oder die Selbstversorgung mit Brennholz in den Vordergrund stellen (s. Franz 2017, S. 28; Feil et al. 2018, S. 25). Nach der Typisierung von Becker und Borchers (2000) lassen sich die BB-Betriebe der Gruppe der „eher wirtschaftlich eingestellten Besitzertypen" zuordnen. Für die befragten Experten, die zumeist Betriebsleiter oder Eigentümer sind, gilt der wirtschaftliche Erfolg als Maxime des unternehmerischen Handelns, um die von ihnen geführten Betriebe in ihrer Lebensfähigkeit zu erhalten.

Die Betriebe wurden hinsichtlich ihrer betrieblichen Zielsetzungen in der Forst- und Landwirtschaft befragt. Im Fokus standen ökonomische Ziele wie Liquiditätssicherung, Vermögenserhalt 
und Rentabilität, gefolgt von den eher sozioökologischen wie Natur- und Umweltschutz, Gesellschaftliche Akzeptanz und Landschaftsgestaltung. Betriebsziele konnten, in den professionell geführten Ertragsforstbetrieben, von den befragten Experten klar benannt werden. Das grundsätzliche Interesse ${ }^{45}$ der Waldeigentümer nach Krott $(2003$, S. 38), das diesen Zielen zu Grunde liegt, ist der Ertragswert des Waldes. Weiterhin formuliert er als gleichrangige Interessen den Bestand der eigenen Herrschaft und den Erhaltenswert des Waldes. Diese Interessen, die jedem Eigentümer immanent sind, treten in der Befragung an den Stellen deutlich hervor, wo Einflussnahmen der Naturschutzverbände oder der öffentlichen Hand als Einschränkung der Eigentumsfreiheit empfunden werden (vgl. Krott 2003, S. 36).

War die Ertragssituation der Forstbetriebe in den der Befragung vorausgegangenen 10 Jahren aufgrund guter Holzpreise und verhältnismäßig störungsarmer Vegetationsperioden durchaus auskömmlich, so zeigen die vergangenen drei Jahre deutliche Grenzen des unternehmerischen Handelns durch Marktstörungen und ungünstige Witterungsbedingungen auf. Die Entwicklung neuer Geschäftsfelder, bzw. die Erschließung neuer Einkommensquellen gewinnt aufgrund des Fehlens der „Brotbaumart “46 Fichte neue Bedeutung. Neben Windkraft im Wald, der Anlage und dem Betrieb von Bestattungswäldern oder der Vermarktung von Ökopunkten für Ausgleichsund Ersatzmaßnahmen werden bspw. auch für die Inwertsetzung von sonstigen Ökosystemleistungen zum wiederholten Male neue Anläufe genommen (Ministerium für Landwirtschaft, Umwelt und Verbraucherschutz Mecklenburg-Vorpommern et al. 2019). Grundsätzliches Problem in der Vermarktung solcher Leistungen sind die Bedingungen der Nichtausschließbarkeit der Nutzung und der Nichtrivalität im Konsum (Samuelson 1954 in Ring 2013, S. 34), die diese Leistungen zu öffentlichen Gütern machen. Für eine Inwertsetzung bedarf es geeigneter Bewertungsverfahren. Klassische Verfahren der Waldbewertung stoßen hier an ihre Grenzen. Eine Bewertung dieser Güter könnte insofern über die von der Gesellschaft erbrachte Wertschätzung bspw. nach dem Konzept des ökonomischen Gesamtwertes erfolgen (Hansjürgens 2012 in Ring 2013, S. 35).

Die im Bereich des Naturschutzes deutliche Dominanz des Ordnungsrechts (Wissenschaftlicher Beirat für Waldpolitik und Wissenschaftlicher Beirat für Biodiversität und Genetische Ressourcen 2020, S. 4), insbesondere in der Umsetzung von Natura 2000-Schutzgebieten (vgl.

\footnotetext{
${ }^{45}$ In der Politikfeldanalyse werden unter Interessen die „Handlungsorientierungen für Individuen oder Gruppen verstanden, die den Nutzen bezeichnen, den das Individuum oder die Gruppe aus einem bestimmten Objekt, etwa dem Wald, ziehen kann" (Krott 2003, S. 5)

${ }^{46}$ vgl. Huber et al. 2013, S. 282.
} 
Niedersächsisches Ministerium für Umwelt, Energie und Klimaschutz 2015), führt in den befragten Betrieben in Verbindung mit bestehenden Rechtsunsicherheiten (Lutter und Paschke 2018, S. 29) zu einer sinkenden Teilnahmebereitschaft an Naturschutzprogrammen jeglicher Art. Dennoch besteht in vielen Betrieben weiterhin die Absicht, die Erbringung ökologischer Leistungen als betriebliche Aufgabe zu verfolgen, sei es auch nur, um weitere ordnungsrechtliche Einschränkungen zu minimieren.

Als erfolgreiches Vertragsnaturschutzprogramm der öffentlichen Hand wurde von den Befragten der Fallbeispielsanalysen im WaVerNa-Projekt das Bayrische VNPWald bezeichnet (vgl. auch Pressemitteilung von Bayrisches Staatsministerium für Umwelt und Verbraucherschutz 02.07.2019). Zwar waren auch hier Probleme hinsichtlich der kontinuierlichen Mittelverfügbarkeit und der betrieblichen Umsetzung sowie der obligatorischen Bindung an die Natura 2000Schutzgebietskulisse vorhanden, dennoch wurde es von den Verantwortlichen als effektiv und zufriedenstellend bewertet. Diese Einschätzung findet sich auch bei Güthler et al. (2018) wieder, die das Programm hinsichtlich der Effektivität und der Inanspruchnahme untersucht haben. Interessanterweise resultiert dieser Erfolg nach Meinung der Autoren auch aus der Abkehr von EU-kofinanzierten Finanzmitteln, was zu einer wesentlichen Vereinfachung der Verwaltungsprozesse geführt hat (Güthler et al. 2018, S. 380). Die komplexen Umsetzungsprozesse europäischer Förderprogramme sorgen auch in der Verwaltung für die Gefahr des Akzeptanzverlusts durch hohe Transaktionskosten (Weber 2015), so dass bei der Vereinfachung von Umsetzungen letztlich beide Seiten von handhabbaren Lösungen profitieren.

Aus dem WaVerNa-Projekt, an dem der Autor dieser Arbeit maßgeblich beteiligt war, wurden nach der Analyse der Hemmnisse Handlungsempfehlungen für eine Neuordnung des Vertragsnaturschutzes im Wald entwickelt (Franz et al. 2018a), die auch von den Wissenschaftlichen Beiräten für Waldpolitik und für Biodiversität und Genetische Ressourcen (2020) in ihrer Stellungnahme für Wege eines effizienteren Waldnaturschutzes in Deutschland aufgegriffen wurden.

Größter Mangel aus Sicht der dem Vertragsnaturschutz im Wald zugewandten BB-Forstbetriebe ist die Kontinuität der Finanzierung. Während in den Fallbeispielsbetrieben für eine längere Ausgestaltung der Finanzierungszeiträume plädiert wurde, ist aus Sicht der BB-Betriebe langfristig vor allem eine Loslösung der Finanzmittel aus dem Haushaltsvorbehalt der Verwaltungen zielführend. In der Stellungnahme der wissenschaftlichen Beiräte wird hierzu die Einrichtung einer Naturschutzstiftung auf Zuwendungsbasis empfohlen, um aus politischer Sicht ein klares langfristiges Bekenntnis für den Vertragsnaturschutz im Wald zu geben und gleichzeitig das Problem des Haushaltsvorbehalts aufzuheben (Wissenschaftlicher Beirat für Waldpolitik und 
Wissenschaftlicher Beirat für Biodiversität und Genetische Ressourcen 2020, S. 41). Mit diesem Vorschlag liegen die Beiräte nahe beim eingangs vorgestellten, stiftungsbasierten hessischen Modell (Hessischer Waldbesitzerverband et al. 2002b). Selbst in der Landwirtschaft werden die siebenjährigen ELER-Förderzeiträume als zu knapp angesehen, um einen langfristigen positiven Effekt auf die naturschutzfachlichen Ziele zu haben und es wird für eine andere Form der Finanzierung plädiert (Fährmann et al. 2018, S. 172). Die Bund/Länderarbeitsgemeinschaft Naturschutz, Landschaftspflege und Erholung (LANA) geht noch einen Schritt weiter und plädiert für die Einrichtung eines EU-weiten Naturschutzfonds, um neben dem Vertragsnaturschutz in Landund Forstwirtschaft auch bspw. Ausgleichszahlungen für Natura 2000-Gebiete und sonstige Arten- und Naturschutzmaßnahmen zu bündeln und von den bisherigen aufwendigen Finanzierungsinstrumenten zu lösen (Bund/Länderarbeitsgemeinschaft Naturschutz, Landschaftspflege und Erholung (LANA) 2016, S. 11). Damit befindet sie sich im Schulterschluss mit dem ThünenInstitut, das sich ebenfalls für ein neues Finanzierungsinstrument ausspricht, das den besonderen Anforderungen der Honorierung von Ökosystemleistungen Rechnung trägt (Elsasser et al. 2020, S. 31) und dem Bundesamt für Naturschutz (Pabst et al. 2018, S. 75). Stärker national orientiert, plädieren auch Güthler et al. (2018, S. 385) in ihrer Analyse des erfolgreichen bayrischen Vertragsnaturschutzprogramms für eine stärkere nationale Beachtung des Themas im Rahmen der GAK und einer besseren Anpassung der aus der Landwirtschaft stammenden Vorgaben an die forstlichen Besonderheiten.

Die oftmals als zu starr und zu wenig an reale Gegebenheiten bewerteten Maßnahmen verursachen den Betrieben Schwierigkeiten in der Umsetzung oder sorgen für einen vollständigen Verzicht auf die Umsetzung der Maßnahme. Die zumeist fest vorgegebenen Anforderungen hinsichtlich der Antragstellung, Umsetzung und Kontrolle zielen aus Behördensicht auf eine Vereinfachung der Verwaltungsprozesse ab. Dieses grundsätzlich nachvollziehbare Vorgehen stellt für die Forstbetriebe eine Hürde dar. Aus dem Festhalten an den Regularien resultiert auf Seiten der Betriebe die Wahrnehmung mangelnder Gesprächsbereitschaft, keiner Gespräche auf Augenhöhe oder auch eines Über-Unterordnungsverhältnisses. Gleichzeitig sehen sich die Betriebe als örtliche Experten, mit dem Sach- und Fachverstand ökologische Werte dort zu sichern, wo die Effizienz am höchsten ist. Dämpfend auf das Engagement für freiwillige Naturschutzmaßnahmen wirken sich die ordnungsrechtlichen Einschränkungen der Natura 2000-Schutzgebietsausweisungen aus (vgl. Nielsen et al. 2018, S. 9). Die marginalen Ausgleichszahlungen schaffen keine gesteigerte Akzeptanz und das Empfinden oktroyierten Naturschutzes resultiert in einer zuneh- 
menden Verweigerungshaltung. Eine Lösung dieses Konflikts hinsichtlich der Unterschutzstellung von Natura 2000-Gebieten ist indes aufgrund der bereits angeführten gerichtlichen Vorgaben im Rahmen dieser Arbeit nicht erkennbar.

Eine Verbesserung der Akzeptanz von Vertragsnaturschutzmaßnahmen könnte durch einen Wandel in der Maßnahmenausgestaltung in den Förderprogrammen hin zu einer verstärkten Ergebnisorientierung erreicht werden. Den Betrieben würde mehr Freiraum, Verantwortung und Handlungsinitiative in der Umsetzung der Maßnahmen gelassen. Dies würde sowohl innerbetriebliche Abläufe verbessern als auch den Programmierungsaufwand auf behördlicher Seite verringern (Wissenschaftlicher Beirat für Waldpolitik und Wissenschaftlicher Beirat für Biodiversität und Genetische Ressourcen 2020, S. 42). ${ }^{47}$ Allerdings bedürfte es der Weiterentwicklung geeigneter Struktur- oder Artindikatoren, um die Ergebnisse messen zu können. Der Schwerpunkt der bisherigen Indikatorenentwicklung liegt auf Strukturelementen, ohne dass daraus automatisch auch auf das Vorkommen von Arten geschlossen werden kann (Paillet et al. 2018). Die befragten Betriebe stehen dabei aus ihrer Erfahrung heraus einer erfolgsorientierten Ausprägung von Vertragsnaturschutzprogrammen skeptisch gegenüber, da ein solches System nur mit reibungsloser Kommunikation funktionieren kann (vgl. Wissenschaftlicher Beirat für Waldpolitik und Wissenschaftlicher Beirat für Biodiversität und Genetische Ressourcen 2020, S. 42), die in vielen Fällen als eher gestört beschrieben wurde (vgl. Sotirov 2017, S. 51). Auch besteht bei Nichteintreten des gewünschten Zielzustands trotz durchgeführter Maßnahmen das erhebliche Risiko der Nichtzahlung (vgl. Bartkowski et al. 2019).

Aus den Ergebnissen der befragten Betriebe, die Vertragsnaturschutz in der Landwirtschaft umgesetzt haben, wird deutlich, dass Agrarumweltmaßnahmen besser an die landwirtschaftlichen Betriebsabläufe angepasst sind als Vertragsnaturschutzmaßnahmen im Wald. Diese bedürfen einer besseren Anpassung an betriebliche Abläufe. Hierbei muss jedoch auch beachtet werden, dass landwirtschaftliche Betriebe im Rahmen der ordnungsgemäßen Landwirtschaft bereits zu einem höheren Dokumentationsaufwand verpflichtet sind als es bei privaten Erwerbsforstbetrieben der Fall ist. Insofern können die Dokumentation- und Kontrollaufwendungen für Vertragsnaturschutzmaßnahmen in der Landwirtschaft besser in bestehende Systeme eingepflegt

\footnotetext{
47 In der Betriebswirtschaftslehre wird ein vergleichbares System, 1954 entwickelt vom US-amerikanischen Ökonom Drucker (1998) als „management by objectives" bezeichnet, in der militärischen Führung ist das Führen mit Auftrag als Methode bereits seit den deutschen Einigungskriegen ab 1864 bekannt (Oetting 1993) und bis heute maßgebliches Instrument in der Inneren Führung der Bundeswehr zur eigenverantwortlichen Zielerreichung. Die Ergebnisorientierung ist insofern ein Instrument, dass dem Handeln ein großes Maß an Eigenverantwortung und Initiative übertragt, aber auch mit entsprechenden Risiken einhergeht.
} 
werden (bspw. Ackerschlagkarteien, etc.). Die Übertragung des Feldblocksystems der Landwirtschaft auf den Wald, in Mecklenburg-Vorpommern zum Zwecke der Ausgleichszahlungen von Natura 2000 durchgeführt (vgl. Ministerium für Landwirtschaft, Umwelt und Verbraucherschutz Mecklenburg-Vorpommern 2016), hat sich dabei als in der Praxis schwer umsetzbar erwiesen, da sie keine Rücksicht auf die bestehenden Bewirtschaftungsstrukturen von Abteilungen und Beständen nimmt (Loë 2017).

Ein interessanter Aspekt der Befragung der BB-Betriebe ist die stärkere Betonung des Betriebsziels Natur- und Umweltschutz in der Landwirtschaft als in der Forstwirtschaft. Dies deutet darauf hin, dass die befragten Eigentümer und Betriebsleiter in der Landwirtschaft bereits stärker sensibilisiert gegenüber der öffentlichen Wahrnehmung ihrer Arbeit sind, bzw. sie einem höheren Rechtfertigungsdruck ausgesetzt sind.

Die Angemessenheit des Entgelts für die Umsetzung von Vertragsnaturschutzmaßnahmen im Wald wird von den beteiligten Parteien sehr unterschiedlich wahrgenommen. Während aus Sicht der Betriebe neben einem ökologischen auch ein ökonomischer Mehrwert einer umgesetzten Maßnahme vorhanden sein muss, verfolgen die öffentlichen Verwaltungen als Geldgeber zumeist das Ziel eines Ertragsausgleiches (s. Bund/Länderarbeitsgemeinschaft Naturschutz, Landschaftspflege und Erholung (LANA) 2018). Allerdings bestehen auch hier innerhalb der gleichen Institution unterschiedliche Auffassungen, wenn einerseits der Misserfolg von Agrarumweltmaßnahmen auf den Mangel von Anreizkomponenten zurückgeführt (LANA 2016, S. 8) und andererseits keine solche in den Fördergrundsätzen für Vertragsnaturschutz im Wald vorgesehen wird (LANA 2018, S.2). Elsasser et al. (2020, S. 30) sehen in einer mangelnden Gewinnerzielungsmöglichkeit auch für die behördliche Seite ein Risiko, da dort hohe Verwaltungskosten für Förderprogramme entstehen, die schlussendlich nicht in Anspruch genommen werden. Mit der Ermittlung von Mindestbeträgen, die für typische Vertragsnaturschutzmaßnahmen im Wald notwendig sind, hat sich ein großer Teil dieser Arbeit befasst. Die Aktualisierung und Erweiterung bestehender Bewertungskonzepte soll nachfolgend erörtert werden.

\subsection{Diskussion der ökonomischen Bewertungskonzepte}

Die vielfältigen Veränderungen der Holzmärkte, der naturalen Gegebenheiten und der politischen und gesellschaftlichen Ansprüche an den Wald haben die Aktualisierung bestehender Bewertungskonzepte notwendig gemacht. Das in 4.3 dargestellte Divergieren von Ertragsniveaus in Laub- und Nadelhölzern sowie die zunehmende Inanspruchnahme des Privatwalds durch Naturschutzeinschränkungen im Rahmen von bspw. Natura 2000 (s. 4.4) unterstreichen die Not- 
wendigkeit aktueller und in der Praxis anwendbarer Bewertungshilfen für politische Entscheidungsträger und Waldeigentümer. Diese beiden Faktoren verschärfen den Konflikt zwischen Ökonomie und Ökologie und erschweren den Waldbesitzern die Abkehr von der Holznutzung als Hauptfinanzierungsquelle ihres betrieblichen Handelns (s. Ermisch et al. 2015 in Wissenschaftlicher Beirat für Waldpolitik und Wissenschaftlicher Beirat für Biodiversität und Genetische Ressourcen 2020, S. 40) hin zu freiwilligen Naturschutzvereinbarungen, bzw. der Inwertsetzung der von ihnen bislang überwiegend kostenfrei zur Verfügung gestellten sonstigen Ökosystemleistungen. Obwohl groß angelegte Forschungsprojekte auf europäischer Ebene diesen Leistungen monetäre Werte zuweisen, die die der Rohholzbereitstellung noch übersteigen, ist jedoch auch deutlich zu erkennen, dass im Gegensatz zur Landwirtschaft nur wenige Ansätze zur Inwertsetzung vorhanden sind (Bösch et al. 2018, S. 82). Für einen langfristigen Erhalt der Biodiversität und des Naturkapitals ist eine konsequente Umsetzung von Finanzierungsprogrammen jedoch unumgänglich (Palahí et al. 2020).

Die beschriebenen Bewertungskonzepte können und müssen für den konkreten Bewertungsfall angepasst werden. Aufgrund der vielen Eingangsparameter lassen sich nur bedingt pauschale Wertbeträge für Ausgleichs- oder Entschädigungsbeträge ermitteln. Diesem Umstand wird in der Förderpraxis bspw. durch dimensionsabhängige Förderbeträge für den Erhalt von Alt- und Habitatbäumen Rechnung getragen (vgl. Bayrisches Staatsministerium für Ernährung, Landwirtschaft und Forstwirtschaft 2014).

Von mindestens ebenso großer Bedeutung wie der Höhe der Entgelte ist auch die konkrete räumliche Umsetzung von Vertragsnaturschutzmaßnahmen in der Fläche. Hier konnte gezeigt werden, dass eine räumliche Konzentration bewirtschaftungserschwerender Maßnahmen, wie z. B. dem Erhalt von Alt- und Habitatbäumen, einen wesentlichen Beitrag zur besseren Einbindung in die betrieblichen Abläufe und den Erhalt von Arbeits- und Verkehrssicherheitsmaßnahmen leisten können. Alt- und Habitatbäume stellen in bewirtschafteten Wäldern ein Schlüsselelement der biologischen Vielfalt dar (Mordini et al. 2012, S. 29). Die Anzahl der Habitatbäume, die in Vertragsnaturschutzprogrammen (s. Bayrisches Staatsministerium für Ernährung, Landwirtschaft und Forsten 2014) oder Schutzgebietsverordnungen (s. Niedersächsisches Ministerium für Umwelt, Energie und Klimaschutz 2015) typischerweise fest vorgegeben ist, muss dabei nicht immer einer ökologischen Zielerreichung folgen. Niedermann-Meier et al. (2013, S. 28) plädieren hingegen für eine flexiblere Gestaltung in Abhängigkeit der ökologischen Wertigkeit der einzelnen Bäume. Die Konzentration der Habitatbäume steht dabei nicht im Widerspruch zu naturschutzfachlichen Zielsetzungen, sondern kann als sog. „Trittsteinkonzept“ einen wichtigen Beitrag zur Biotopvernetzung leisten (vgl. Jedicke 1990). Die räumliche Konzentration dient 
aus betrieblicher Sicht vorrangig der Vermeidung von ganzflächigen Arbeitsschutz- und Verkehrssicherungsrisiken. Die Waldbesitzer haften zwar nicht für die sogenannten waldtypischen Gefahren, die sich aus der Natur oder der ordnungsgemäßen Bewirtschaftung des Waldes unter Beachtung der jeweiligen Zweckbestimmung ergeben, jedoch haften sie für Gefahren, die künstlich geschaffen oder geduldet werden (Landesbetrieb ForstBW 2015, S. 7).$^{48}$ Auch wenn aus einer natürlichen Entwicklung von Bäumen keine besondere Verkehrssicherungspflicht erwächst, muss dem Vorsorgeprinzip entsprechend auf eine Ausweisung in Gefährdungsbereichen, wie z.B. Straßen- oder Wanderwegnähe verzichtet werden (Landesbetrieb ForstBW 2015, S. 11). Für den Umgang mit der Verkehrssicherungspflicht ist von wichtiger Bedeutung, dass diese grundsätzlich an Dritte übertragbar ist (aid infodienst Ernährung, Landwirtschaft, Verbraucherschutz e.V. i.L. 2016, S. 65). Somit ließe sich im Zuge der Verhandlung von Vertragsnaturschutzmaßnahmen ein Teil der anfallenden betrieblichen Risiken auf den Vertragspartner übertragen.

Im Rahmen der Fallstudie Hämeler Wald wurden die aktualisierten Bewertungskonzepte einer Anwendung in der Praxis unterzogen, um die Bewirtschaftungseinschränkungen der Natura 2000-Schutzgebietsausweisung aufzuzeigen. Auch wenn es sich im konkreten Fall nicht um ein Vertragsnaturschutzbeispiel handelte, stehen Natura 2000 und der Vertragsnaturschutz im Wald doch in unmittelbarem Zusammenhang. So ist bspw. die Umsetzung von Maßnahmen des Bayrischen Vertragsnaturschutzprogramm an die Gebietskulisse Natura 2000 gebunden (Bayrisches Staatsministerium für Ernährung, Landwirtschaft und Forsten 2014, S. 5). Auch wenn der Erreichung des Schutzzwecks in Natura 2000-Gebieten durch den Abschluss von Naturschutzverträgen gerichtlich enge Grenzen gesetzt worden sind (vgl. EuGH, Urteil vom 25.11.1999), besteht nach Bundesnaturschutzgesetz die bereits ausgeführte vorrangige Prüfpflicht von vertraglichen Vereinbarungen als milderes Mittel im Gegensatz zu ordnungsrechtlichen Auflagen. Die ökonomischen Einschränkungen der Forstbetriebe durch die Schutzgebietsverordnungen belasten die Betriebe stark und können auf den betroffenen Flächen zur Unwirtschaftlichkeit führen. Dies steht im Widerspruch zu der dem Natura 2000-Schutzgebietsnetz zugrunde liegenden europäischen FFH-Richtlinie, die bereits in der Präambel eine Berücksichtigung der wirtschaftlichen Anforderungen bei der Förderung der biologischen Vielfalt formuliert ist (Rat der Europäischen Gemeinschaften 1992). Diesen Widerspruch aufzuzeigen und den beteiligten Akteuren bessere Bewertungsgrundlagen für die Zukunft an die Hand zu geben, war Ziel der Fallstudie.

\footnotetext{
${ }^{48}$ Siehe auch: Bundesgerichtshof, Urteil vom 02.10.2012.
} 


\section{Schlussfolgerungen \& Ausblick}

Vertragsnaturschutz im Wald stellt trotz aller politischen Absichtserklärungen und wissenschaftlicher Forschungsergebnisse bis heute nur eine Randerscheinung im Wald und der Naturschutzlandschaft dar. Während sich der Vertragsnaturschutz in der Landwirtschaft aufgrund der guten Einbindung in betriebliche Abläufe und angemessenen Entgeltzahlungen als erfolgreiches Naturschutzinstrument entwickelt hat, sind die Hemmnisse im Wald nach wie vor vielfältig.

Eine bundesweite Befragung von Forstbetrieben zeigt einerseits zwar ein großes Interesse, andererseits allerdings eine Vielzahl von Problemen in der Umsetzung und der langfristigen Ausgestaltung dieses Instruments. Tiefergehende Analysen in Betrieben, die seit einiger Zeit solche Maßnahmen in ihren Wäldern umgesetzt haben, bestätigen die Annahme, dass Vertragsnaturschutz im Wald unter den aktuellen Vorzeichen für keine Seite erfolgreich umgesetzt werden kann. Zu groß sind mittlerweile die negativen Erfahrungen auf Seiten der Betriebe mit mangelnder Finanzausstattung, zu aufwendigen Verfahren und rechtlichen Unklarheiten. Gleichzeitig sorgt die Nichtinanspruchnahme von angebotenen Förderinstrumenten zu nachlassendem Engagement der öffentlichen Verwaltungen zur Programmierung aufwendiger, EU-kofinanzierter Förderprogramme, die mit hohen Transaktionskosten verbunden sind.

Die Befragung einer großen Gruppe von Erwerbsforstbetrieben, die zum Teil auch landwirtschaftliche Betriebe sind, bestärkt die Überzeugung, dass eine Fortführung bestehender Vertragsnaturschutzansätze im Wald nur bedingt zielführend ist. Zu groß sind die schlechten Erfahrungen in der Vergangenheit und zu groß der wahrgenommene Zugriff auf das Eigentum durch ordnungsrechtliche Naturschutzvorgaben. Belastete Kommunikation und mangelnde Anerkennung der in den Betrieben vorhandenen Expertise haben zu großer Frustration geführt.

Die Biodiversitätsstrategie der Bundesregierung sieht die Umsetzung von Vertragsnaturschutz auf $10 \%$ der deutschen Privatwaldfläche vor. Dieses Ziel ist aktuell bei weitem noch nicht erreicht. Es bedarf neuer Programme, die unter Berücksichtigung der aufgezeigten Hemmnisse das für den Erhalt der Biodiversität und die Kooperation der privaten Forstbetriebe so wichtige Instrument attraktiv gestalten.

Die Aktualisierung und Erweiterung bestehender Bewertungskonzepte für forstliche Nutzungseinschränkungen liefert den Beteiligten ein wichtiges Handwerkszeug zur verbesserten Kommunikation und Transparenz. Für konkrete Bewertungsfälle lassen sich nachvollziehbare Ausgleichsbeträge ermitteln, die im Rahmen einer Verhandlung als Mindestpreis für die Umsetzung 
von Vertragsnaturschutzmaßnahmen verwendet werden können. Dabei müssen Einschränkungen betrieblicher Abläufe und Aspekte der Verkehrssicherung eine besondere Berücksichtigung erfahren. Die bestehenden Rechtsunsicherheiten hinsichtlich Haftungsrisiken und besonders des Artenschutzes bleiben bestehen und können nur durch eine Anpassung der rechtlichen Grundlagen gelöst werden.

Diese Arbeit hofft, einen Beitrag für ein besseres Verständnis der Situation der beteiligten Akteure liefern zu können. Nur wenn Transparenz und das notwendige, ökonomische Handwerkszeug allen Beteiligten zur Verfügung stehen, kann der Vertrag im Sinne des „Vertragens" zum erfolgreichen Abschluss gebracht werden. Dem Autor ist jedoch wohl bewusst, dass es weiterer Forschung aber insbesondere politischer Entscheidungen hinsichtlich der langfristigen Finanzierungsmöglichkeiten, der Klärung rechtlicher Unsicherheiten und der Vermeidung hoher Transaktionskosten seitens der Verwaltungen bedarf, um den Vertragsnaturschutz im Wald zum Erfolg zu führen. Besonders vor dem Hintergrund der großen Waldschäden und der laufenden Debatte um die Klimaschutzleistung des deutschen Waldes kann ein neuartig gestalteter Vertragsnaturschutz einen wichtigen Beitrag zur Erfüllung der mannigfaltigen Ansprüche an den Wald leisten. 


\section{Selbstständigkeitserklärung}

Ich versichere, dass ich die vorliegende schriftliche wissenschaftliche Abhandlung selbständig verfasst und keine anderen als die angegebenen Quellen und Hilfsmittel benutzt habe. Weiterhin wurde anderweitig keine entsprechende Promotion beantragt und hierbei die eingereichte Dissertation oder Teile daraus vorgelegt. 


\section{Literaturverzeichnis}

Abelshauser, Werner (2003): Kulturkampf. Der deutsche Weg in die neue Wirtschaft und die amerikanische Herausforderung. Berlin: Kulturverl. Kadmos (Kulturwissenschaftliche Interventionen, 4). Online verfügbar unter http://hsozkult.geschichte.hu-berlin.de/rezensionen/2003-4-012.

aid infodienst Ernährung, Landwirtschaft, Verbraucherschutz e.V. i.L. (Hg.) (2016): Verkehrssicherungspflicht der Waldbesitzer. 3. Aufl. Bonn (1588/2016), zuletzt geprüft am 08.12.2020.

Atteslander, Peter (2010): Methoden der empirischen Sozialforschung. 13., neu bearbeitete und erweiterte Auflage. Berlin: Erich Schmidt Verlag (ESV basics).

Bartkowski, Bartosz; Droste, Nils; Ließ, Mareike; Sidemo-Holm, William; Weller, Ulrich; Brady, Mark V. (2019): Implementing result-based agri-environmental payments by means of modelling. Helmholtz-Zentrum für Umweltforschung - UFZ. Leipzig, zuletzt geprüft am 02.12.2020.

Bayrische Staatsforsten (Hg.) (2013): Naturschutzkonzept für den Forstbetrieb Rothenbuch.

Bayrisches Staatsministerium für Ernährung, Landwirtschaft und Forsten (2014): Richtlinie über Zuwendungen nach dem Bayerischen Vertragsnaturschutzprogramm Wald. VNPWaldR 2015. Online verfügbar unter https://eur-lex.europa.eu/LexUriServ/LexUriServ.do?uri=CONSLEG:1992L0043:20070101:DE:PDF.

Bayrisches Staatsministerium für Ernährung, Landwirtschaft und Forstwirtschaft (2014): Richtlinie über Zuwendungen nach dem Bayrischen Vertragsnaturschutzprogramm Wald. VNPWaldR 2015, vom 20.10.2014. Online verfügbar unter https://www.stmelf.bayern.de/mam/cms01/wald/waldbesitzer/dateien/vnpwaldr_2015.pdf, zuletzt geprüft am 29.01.2020.

Bayrisches Staatsministerium für Umwelt und Verbraucherschutz (02.07.2019): Bayern: Neuer Rekord beim Vertragsnaturschutz im Wald. Online verfügbar unter www.forstpraxis.de.

Becker, Gero; Borchers, Jens (2000): Strukturen und Motive der Privatwaldbesitzer in NordrheinWestfalen als Basis zur Entwicklung alternativer Nutzungskonzepte und neuer forstwirtschaftspolitischer Instrumente. Freiburg.

Blomberg, Georg Moritz Freiherr von (2015): Analyse von Prämienzahlungen für Agrarumweltmaßnahmen und mögliche Folgerungen für Vertragsnaturschutz im Wald. Analysis of premium payments for agri-environment-climate measures and possible consequences for contractual conservation assignments in the forest. Masterarbeit. Georg-August-Universität, Göttingen. Abteilung Forstökonomie und Forsteinrichtung.

Blomberg, Georg Moritz Freiherr von; Möhring, Bernhard (2018): Vertragsnaturschutz aus forstbetrieblicher Sicht. In: AFZ Der Wald (21), 24-27.

Blomberg, Georg Moritz Freiherr von; Volckens, Frederik; Möhring, Bernhard (2019): Ergebnisse aus dem BB-Betriebsvergleich 2018. In: AFZ Der Wald (23), S. 21-27.

Böckmann, Thomas (2016): Warum sind Betriebsinventuren für die forstliche Praxis wichtig? In: Forstarchiv (87), S. 31-37. DOI: 10.4432/0300-4112-87-31.

Bormann, Kristin (2015): Faktencheck ELER-Förderung - Forstliche Förderung. Thünen-Institut. Braunschweig (Thünen Working Paper, 10).

Bösch, Matthias; Elsasser, Peter; Franz, Kristin; Lorenz, Martin; Moning, Christoph; Olschewski, Roland et al. (2018): Forest ecosystem services in rural areas of Germany: Insights from 
the national TEEB study. In: Ecosystem Services 31, S. 77-83. DOI: 10.1016/j.ecoser.2018.03.014.

Bund/Länderarbeitsgemeinschaft Naturschutz, Landschaftspflege und Erholung (LANA) (Hg.) (2016): Wirksamkeit der derzeitigen EU-Naturschutzfinanzierung in Deutschland und Anforderungen für die nächste Förderperiode ab 2020. Positionspapier der LANA-Expertengruppe "EU-Naturschutzfinanzierung / GAP 2020".

Bund/Länderarbeitsgemeinschaft Naturschutz, Landschaftspflege und Erholung (LANA) (Hg.) (2018): Fördergrundsatz Vertragsnaturschutz im Wald. Mainz.

Bundesgerichtshof, Urteil vom 02.10.2012, Aktenzeichen VI ZR 311/11.

Bundesminister der Justiz (1986): Bekanntmachung der Richtlinien für die Begründung von Nutzungsverhältnissen an Waldflächen für Zwecke der Verteidigung. NVWald. In: Bundesanzeiger, zuletzt geprüft am 18.11.2020.

Bundesministerium für Ernährung und Landwirtschaft (2014a): Nationale Rahmenregelung. NRR, zuletzt geprüft am 18.11.2020.

Bundesministerium für Ernährung und Landwirtschaft (2014b): Verordnung über die Einhaltung von Grundanforderungen und Standards im Rahmen unionsrechtlicher Vorschriften über Agrarzahlungen. AgrarZahIVerpflV.

Bundesministerium für Ernährung und Landwirtschaft (Hg.) (2019): Rahmenplan der Gemeinschaftsaufgabe "Verbesserung der Agrarstruktur und des Küstenschutzes" 2019-2022. Bonn. Online verfügbar unter https://www.bmel.de/SharedDocs/Downloads/Landwirtschaft/Foerderung/Rahmenplan2019-2022.pdf;jsessionid=D257C719A4D09E2F16F10B2229BCF0C1.1_cid385?_blob=publicationFile, zuletzt geprüft am 30.01.2020.

Bundesministerium für Umwelt, Naturschutz und Reaktorsicherheit (BMU) (Hg.) (2007): Nationale Strategie zur biologischen Vielfalt. Kabinettsbeschluss vom 7. November 2007. Berlin, zuletzt geprüft am 01.12.2020.

Bundesministerium für Umwelt, Naturschutz und Reaktorsicherheit (BMU) (Hg.) (2017): Biologische Vielfalt in Deutschland. Rechenschaftsbericht 2017. Berlin.

Buren, Guillaume de; Lieberherr, Eva; Steinmann, Kathrin (2016): Waldspezifische Förderung in der Europäischen Union: Umsetzung in drei Fallbeispielen. In: Schweizerische Zeitschrift fur Forstwesen 167 (5), S. 270-277. DOI: 10.3188/szf.2016.0270.

Deutscher Bundestag (2009): Gesetz über Naturschutz und Landschaftspflege. BNatSchG, vom 15.09.2017.

Deutscher Bundestag (11.10.2016): Gesetz über die Gemeinschaftsaufgabe "Verbesserung der Agrarstruktur und des Küstenschutzes, GAK-Gesetz, vom 11.10.2016. In: Bundesgesetzblatt.

Drucker, Peter F. (1998): Praxis des Managements. Ein Leitfaden für die Führungs-Aufgaben in der modernen Wirtschaft. Unveränd. Nachdr. der Ausg. 1969 (6. Aufl.). Düsseldorf: ECON.

Elmer, Michael; Bieker, Dirk; Linnemann, Britta (2018): Förderung von Alt- und Totholz im privaten Wirtschaftswald. In: AFZ Der Wald (2), S. 16-18, zuletzt geprüft am 19.11.2020.

Elsasser, Peter; Köthke, Margret; Dieter, Matthias (2020): Ein Konzept zur Honorierung der Ökosystemleistungen der Wälder. Thünen-Institut. Hamburg (Thünen Working Paper, 152), zuletzt geprüft am 02.12.2020. 
Entenmann, S.; Schaich, Harald (2014): Natura 2000 im Privatwald. Umsetzungsmöglichkeiten durch die EU-Naturschutzfinanzierung,. Hg. v. Naturschutzbund Deutschland (NABU) e.V. Berlin.

EuGH, Urteil vom 25.11.1999, Aktenzeichen C-96/98.

Europäische Kommission; Europäisches Parlament (17.12.2013): Verordnung (EU) Nr. 1305/2013 des Europäischen Parlaments und des Rates vom 17. Dezember 2013 über die Förderung der ländlichen Entwicklung durch den Europäischen Landwirtschaftsfonds für die Entwicklung des ländlichen Raums (ELER) und zur Aufhebung der Verordnung (EG) Nr. 1698/2005, ELER-VO 1305/2013. In: Amtsblatt der Europäischen Union.

Europäisches Parlament; Rat der Europäischen Union (2013a): Verordnung (EU) Nr. 1305/2013 des Europäischen Parlaments und des Rates vom 17. Dezember 2013 über die Förderung der ländlichen Entwicklung durch den Europäischen Landwirtschaftsfonds für die Entwicklung des ländlichen Raums (ELER) und zur Aufhebung der Verordnung (EG) Nr. 1698/2005. EU VO 1305/2013.

Europäisches Parlament; Rat der Europäischen Union (2013b): Verordnung (EU) Nr. 1307/2013 des Europäischen Parlaments und des Rates vom 17. Dezember 2013 mit Vorschriften über Direktzahlungen an Inhaber landwirtschaftlicher Betriebe im Rahmen von Stützungsregelungen der Gemeinsamen Agrarpolitik und zur Aufhebung der Verordnung (EG) Nr. 637/2008 des Rates und der Verordnung (EG) Nr. 73/2009 des Rates. EU VO 1307/2013, zuletzt geprüft am 18.11.2020.

Fährmann, Barbara; Bergschmidt, Angela; Bathke, Manfred; Eberhardt, Winfried; Ebers, Henrik; Fengler, Birgit et al. (2018): PFEIL - Programm zur Förderung im ländlichen Raum 2014 bis 2020 in Niedersachsen und Bremen. Analyse der Inanspruchnahme und Umsetzung. Hg. v. Thünen-Institut. Thünen-Institut. Hamburg.

Feil, Philine; Neitzel, Christoph; Seintsch, Björn; Dieter, Matthias (2018): Privatwaldeigentümer und gesellschaftliche Ansprüche. In: AFZ Der Wald (5), S. 24-27.

Franz, Kristin (2017): Zahlungen für Naturschutz im Wald. Problem und Lösungsansatz aus Sicht des Agency-Ansatzes. Dissertation. Georg-August-Universität, Göttingen, zuletzt geprüft am 25.11.2020.

Franz, Kristin; v. Blomberg, Georg Moritz Freiherr; Demant, Laura; Dieter, Matthias; Lutter, Carina; Meyer, Peter et al. (2018a): Perspektiven für den Vertragsnaturschutz. In: AFZ Der Wald (21), S. 30-33.

Franz, Kristin; v. Blomberg, Georg Moritz Freiherr; Demant, Laura; Lutter, Carina; Seintsch, Björn; Selzer, Anne M. (2018b): Umsetzung von Vertragsnaturschutz im deutschen Wald. In: AFZ Der Wald (21), S. 13-15.

Freese, Jan (2016): Überblick über die Waldumweltmaßnahmen. Vortrag. Deutscher Naturschutztag. Magdeburg, 2016. Online verfügbar unter https://docplayer.org/123126242Ueberblick-ueber-die-waldumweltmassnahmen.html, zuletzt geprüft am 11.05.2021.

Freese, Jan; Klimek, Sebastian; Marggraf Rainer (2011): Auktionen und ergebnisorientierte Honorierung bei Agrarumweltmaßnahmen. In: Natur und Landschaft 86 (4), S. 156-159.

Frenz, Walter (2011): Vertragsnaturschutz in neuem Gewand. In: NuR 33 (4), S. 257-265. DOI: 10.1007/s10357-011-2050-x.

Gerstlberger, Wolfgang; Schneider, Karsten (2008): Öffentlich Private Partnerschaften. Zwischenbilanz, empirische Befunde und Ausblick. 1. Auflage. Baden-Baden: Nomos Verlagsgesellschaft mbH \& Co. KG. 
Gläser, Jochen; Laudel, Grit (2010): Experteninterviews und qualitative Inhaltsanalyse als Instrumente rekonstruierender Untersuchungen. 4. Auflage. Wiesbaden: VS Verlag (Lehrbuch). Online verfügbar unter http://d-nb.info/1002141753/04.

Grohe, Simon (2010): Förderung von Waldumweltmaßnahmen aus dem Fonds zur Förderung der Entwicklung des ländlichen Raumes (ELER). Am Beispiel der NABU-Stiftung Nationales Naturerbe in den Ländern Mecklenburg-Vorpommern und Niedersachsen. München: GRIN Verlag GmbH.

Güthler, Wolfram; Market, Renate; Häusler, Andreas; Dolek, Matthias (2005): Vertragsnaturschutz im Wald Bundesweite Bestandsaufnahme und Auswertung. Hg. v. Bundesamt für Naturschutz. Bonn (BfN-Skripten, 146).

Güthler, Wolfram; Müller, Jörg; Gindele-Glaser, Mirjam (2018): Vertragsnaturschutz Wald. Wie erfolgreich ist der kooperative Naturschutz im Wald in Bayern? In: Naturschutz und Landschaftsplanung (50), S. 380-385, zuletzt geprüft am 25.11.2020.

Hansjürgens, Bernd; Lienhoop, Nele; Matzdorf, Bettina; Schröter, Barbara; Schröter-Schlaack, Christoph; Stöcker, Ulrich et al. (2019): Ökosystemleistungen und deren Inwertsetzung in ländlichen Räumen unter Einbeziehung von Klimaaspekten. Hg. v. Bundesamt für Naturschutz. Bonn, zuletzt geprüft am 01.12.2020.

Hessischer Waldbesitzerverband; Hessisches Ministerium für Umwelt, Landwirtschaft und Forsten; Hessischer Städte- und Gemeindebund (Hg.) (2002a): Anlagen zum Rahmenvertrag Naturschutz im Wald. Anlagen 1 - 4. Wiesbaden. Online verfügbar unter https://umwelt.hessen.de/sites/default/files/HMUELV/anlagen_rahmenvertrag_naturschutz_im_wald.pdf, zuletzt geprüft am 29.01.2020.

Hessischer Waldbesitzerverband; Hessisches Ministerium für Umwelt, Landwirtschaft und Forsten; Hessischer Städte- und Gemeindebund (Hg.) (2002b): Rahmenvertrag Naturschutz im Wald. Wiesbaden. Online verfügbar unter https://umwelt.hessen.de/sites/default/files/HMUELV/rahmenvertrag_naturschutz_im_wald.pdf, zuletzt geprüft am 29.01.2020.

Hessisches Ministerium für Umwelt, Landwirtschaft und Forsten; Hessischer Waldbesitzerverband; Hessischer Städte- und Gemeindebund; Hessischer Städtetag (Hg.) (2002): Rahmenvertrag Naturschutz im Wald.

Huber, Wolfgang; Schwarzbauer, Peter; Stern, Tobias (2013): Analyse der Motive österreichischer Kleinwaldeigentümer als Schlüssel für die Holzmobilisierung. In: Schweizerische Zeitschrift fur Forstwesen 164 (9), S. 278-284. DOI: 10.3188/szf.2013.0278.

Jackob, Nikolaus; Schoen, Harald; Zerback, Thomas (Hg.) (2009): Sozialforschung im Internet. Methodologie und Praxis der Online-Befragung. 1. Aufl. Wiesbaden: VS Verlag für Sozialwissenschaften / GWV Fachverlage GmbH Wiesbaden. Online verfügbar unter http://www.socialnet.de/rezensionen/isbn.php?isbn=978-3-531-16071-9.

Jedicke, Eckhard (1990): Biotopverbund. Grundlagen und Maßnahmen einer neuen Naturschutzstrategie. Stuttgart: Ulmer (Ulmer-Fachbuch Landespflege und Naturschutz).

Johann Heinrich von Thünen-Institut; Nordwestdeutsche Forstliche Versuchsanstalt; Georg-August-Universität Göttingen; Universität Hamburg (Hg.) (2019): Gemeinsamer Schlussbericht zum Verbundvorhaben Vertragsnaturschutz im Wald. Analyse der waldökologischen, ökonomischen und rechtlichen Optionen (WaVerNa-Projekt). Hamburg.

Johansson, Per-Olov; Löfgren, Karl-Gustaf (1985): The Economics of Forestry and Natural Resources. New York, Oxford: Basil Blackwell. 
Kettunen, M. (2017): Integration approach to EU biodiversity financing: evaluation of results and analysis of options for the future. Final report for the European Commission (DG ENV) (Project ENV.B.3/ETU/2015/0014. Hg. v. Institute for European Environmental Policy (IEEP). Brüssel/London. Online verfügbar unter https://ec.europa.eu/environment/nature/natura2000/financing/docs/Kettunen_2017_financing_biodiversity.pdf, zuletzt geprüft am 28.01.2020.

Kownatzki, Dierk; von Blomberg, Georg Moritz Freiherr; Demant, Laura; Lutter, Carina; Meyer, Peter; Möhring, Bernhard et al. (2017): Ergebnisse einer Befragung von Forstbetrieben. Status quo der Umsetzung von Naturschutz im Wald gegen Entgelt in Deutschland. Hg. v. Thünen-Institut. Braunschweig (Thünen Working Paper, 81).

Kromrey, Helmut (2009): Empirische Sozialforschung. 12. Aufl. Stuttgart: Lucius \& Lucius Verlagsgesellschaft mbH; Leske + Budrich.

Krott, Max (2003): Politikfeldanalyse Forstwirtschaft. Eine Einführung für Studium und Praxis. 1. Aufl. Stuttgart: Ulmer.

Krott, Max; Suda, Michael (2001): Befragung als Methode der Sozialforschung in der Forstwissenschaft. Frankfurt am Main: Sauerländer (Schriften aus der Forstlichen Fakultät der Universität Göttingen und der Niedersächsischen Forstlichen Versuchsanstalt, 132).

Krüger, Dirk; Parchmann, Ilka; Schecker, Horst (2014): Methoden in der naturwissenschaftsdidaktischen Forschung. Berlin, Heidelberg: Springer Berlin Heidelberg, zuletzt geprüft am 30.04.2019.

Krüger, Heinz-Hermann (2013): Qualitative Forschung in der Erziehungswissenschaft. In: Ingrid Gogolin, Harm Kuper, Heinz-Hermann Krüger und Jürgen Baumert (Hg.): Stichwort: Zeitschrift für Erziehungswissenschaft. Wiesbaden: Springer VS, S. 53-75.

Landesbetrieb ForstBW (Hg.) (2015): Leitfaden zur Verkehrssicherungspflicht. Stuttgart, zuletzt geprüft am 08.12.2020.

Landesbetrieb ForstBW (Hg.) (2016): Alt- und Totholzkonzept Baden-Württemberg. Stuttgart, zuletzt geprüft am 19.11.2020.

Landwirtschaftskammer Niedersachsen (Hg.) (2019): Informationsbroschüre über die einzuhaltenden Verpflichtungen bei Cross Compliance, zuletzt geprüft am 28.01.2020.

Loë, Roderich Freiherr von (2017): Fallstudie Vertragsnaturschutz im Wald. Gotthun, 20.06.2017. Interview an Bernhard Möhring, Laura Demant, Georg Moritz Freiherr von Blomberg, Carina Lutter und Anne Mira Selzer.

Lutter, Carina; Paschke, Marian (2018): Rechtliche Einordnung von Vertragsnaturschutz im Wald. In: AFZ Der Wald (21), S. 28-29.

Mährlein, Albrecht (2017): Einflussnahme von Naturschutzmaßnahmen auf Verkehrs- und Beleihungswerte landwirtschaftlicher Flächen. 2. Deutsches Hochschulforum. Soest, 2017, zuletzt geprüft am 09.12.2020.

Mayring, Philipp (2008): Einführung in die qualitative Sozialforschung. Eine Anleitung zu qualitativem Denken. 5. Aufl. Weinheim, Basel: Beltz (Beltz Studium).

Meier, Sarah (2009): Beurteilung des ökologischen und ökonomischen Werts von Habitatbäumen in Wirtschaftswäldern am Beispiel des Forstbetriebs Baden. Masterarbeit. Eidgenössische Technische Hochschule, Zürich. Departement Umweltwissenschaften, zuletzt geprüft am 18.11.2020. 
Meuser, Michael; Nagel, Ulrike (1991): ExpertInneninterviews - vielfach erprobt, wenig bedacht. ein Beitrag zur qualitativen Methodendiskussion. In: Qualitativ-empirische Sozialforschung : Konzepte, Methoden, S. 441-471, zuletzt geprüft am 29.04.2019.

Mieg, Harald A.; Näf, Matthias (2006): Experteninterviews in den Umwelt- und Planungswissenschaften. Eine Einführung und Anleitung. Lengerich: Pabst Science Publ. Online verfügbar unter http://deposit.d-nb.de/cgi-bin/dokserv id=2875666\&prov=M\&dok_var=1\&dok_ext=htm.

Minister für Umwelt, Raumordnung und Landwirtschaft Nordrhein-Westfalen (1987): Tarifvertrag über die Entlohnung von Holzerntearbeiten nach dem Erweiterten Sortentarif. EST.

Ministerium für Landwirtschaft, Umwelt und Verbraucherschutz Mecklenburg-Vorpommern (2016): Richtlinie über den Erschwernisausgleich für Wald in Natura 2000.Gebieten im Rahmen des Europäischen Landwirtschaftsfonds für die Entwicklung des ländlichen Raums. Wald EARL M-V, zuletzt geprüft am 19.11.2020.

Ministerium für Landwirtschaft, Umwelt und Verbraucherschutz Mecklenburg-Vorpommern; Waldbesitzerverband Mecklenburg-Vorpommern; Arbeitsgemeinschaft Kommunalwald (Hg.) (2019): Gemeinsame Schweriner Erklärung. Wald neu denken - Wald neu lenken. Schwerin, zuletzt geprüft am 03.12.2020.

Möhring, Bernhard (2010): Bewertungskonzept für Einzelbäume. In: AFZ Der Wald 2010 (4), S. 10-13.

Möhring, Bernhard; Rüping, Ursula (2006): Bewertungskonzept für forstliche Nutzungsbeschränkungen. Frankfurt am Main: J.D. Sauerländerś (Schriften zur Forstökonomie, Band 32).

Möhring, Bernhard; Rüping, Ursula; Freiherr von Blomberg, Georg Moritz (2017): Bewertungskonzept für forstliche Nutzungsbeschränkungen. 2., aktualisierte und erweiterte Auflage Band 32. Frankfurt am Main: J.D. Sauerländer's Verlag (Schriften zur Forst- und Umweltökonomie, Band 45).

Möhring, Bernhard; Rüping, Ursula; Leefken, Georg; Ziegeler, Markus (2006): Die Annuität - Ein "missing link" der Forstökonomie. The Annuity - a "missing link" of the Forest Economy. In: Allgemeine Forst- und Jagdzeitung 177 (2), S. 21-29.

Moog, Martin; Brabänder, Horst Dieter (1994): Vertragsnaturschutz in der Forstwirtschaft. Situationsanalyse, Entscheidungshilfen und Gestaltungsvorschläge. 2., unveränd. Aufl. Frankfurt am Main: Sauerländer (Schriften zur Forstökonomie, 3).

Mordini, Manuela; Lehner, Jonas; Niedermann-Meier, Sarah; Nussbeck, Isabelle; Kurschat, Regina; Mühlethaler, Urs (2012): Biotopbäume und Totholz: Forstbetriebliche Auswirkungen. Auslegeordnung und erste methodische Ansätze. Projektbericht im Auftrag des BAFU. Hochschule für Agrar-, Forst- und Lebensmittelwissenschaften HAFL. Zollikofen, zuletzt geprüft am 09.12.2020.

Moxter, Adolf (1983): Grundsätze ordnungsmäßiger Unternehmensbewertung. Zweite, vollständig umgearbeitete Auflage. Wiesbaden: Gabler Verlag.

Nagel, Jürgen (2009): Waldwachstumssimulation mit dem Java Software Paket TreeGrOSS. NWFVA. Göttingen.

Niedermann-Meier, Sarah; Mordini, Manuela; Bütler Sauvain, Rita; Rotach, Peter (2013): Habitatbäume im Wirtschaftswald. In: Wald und Holz (3), S. 26-28, zuletzt geprüft am 18.11.2020. 
Niedersächsische Landesregierung (2016): Verordnung über den Erschwernisausgleich für Wald in geschützten Teilen von Natur und Landschaft in Natura 2000-Gebieten, EA-VO-Wald. In: Niedersächsisches Gesetz- und Verordnungsblatt, zuletzt geprüft am 06.12.2020.

Niedersächsisches Ministerium für Ernährung, Landwirtschaft und Verbraucherschutz (2014): Programm für die Entwicklung im ländlichen Raum. PFEIL.

Niedersächsisches Ministerium für Ernährung, Landwirtschaft und Verbraucherschutz (2018): Richtlinie über die Gewährung von Zuwendungen zur Förderung forstwirtschaftlicher Maßnahmen im Land Niedersachsen, vom 01.05.2018. Online verfügbar unter https://www.bmel.de/SharedDocs/Downloads/Broschueren/FoerderungLandwUnternehmen2020.pdf;jsessionid=D257C719A4D09E2F16F10B2229BCF0C1.1_cid385?_blob=publicationFile, zuletzt geprüft am 30.01.2020.

Niedersächsisches Ministerium für Ernährung, Landwirtschaft und Verbraucherschutz (Hg.) (2019): Die niedersächsische Landwirtschaft in Zahlen. Hannover, zuletzt geprüft am 09.12.2020.

Niedersächsisches Ministerium für Landwirtschaft und Ernährung (2014): Waldbewertungsrichtlinien. WBR 2014, vom 18.12.2013.

Niedersächsisches Ministerium für Umwelt, Energie und Klimaschutz (2015): Unterschutzstellung von Natura 2000-Gebieten im Wald durch Naturschutzgebietsverordnung, zuletzt geprüft am 18.11.2020.

Nielsen, Anne Sofie Elberg; Jacobsen, Jette Bredahl; Strange, Niels (2018): Landowner participation in forest conservation programs: A revealed approach using register, spatial and contract data. In: JFE 30, S. 1-12. DOI: 10.1016/j.jfe.2017.10.003.

Niens, Christine; Marggraf, Rainer (2010): Handlungsempfehlungen zur Steigerung der Akzeptanz von Agrarumweltmaßnahmen - Ergebnisse einer Befragung von Landwirten und Landwirtinnen in Niedersachen. In: Berichte über Landwirtschaft (88), S. 5-36.

Oetting, Dirk W. (1993): Auftragstaktik. Geschichte und Gegenwart einer Führungskonzeption. Frankfurt am Main: Report-Verl.

Oppermann, Rainer (2013): Weiterentwicklung der Agrarumweltprogramme. Maßnahmen zur effektiven Förderung der Biodiversität in der Landwirtschaft bis 2020. Hg. v. Naturschutzbund Deutschland (NABU) e.V. Berlin, zuletzt geprüft am 18.11.2020.

Pabst, Holger; Achtermann, Bea; Langendorf, Ursula; Horlitz, Thomas; Schramek, Jörg (2018): Biodiversitätsförderung im ELER (ELERBiodiv). Endbericht des gleichnamigen Forschungs- und Entwicklungsvorhabens (FKZ 3515880 300). Institut für ländliche Strukturforschung. Frankfurt a.M., zuletzt geprüft am 08.12.2020.

Paillet, Yoan; Archaux, Frédéric; Du Puy, Solène; Bouget, Christophe; Boulanger, Vincent; Debaive, Nicolas et al. (2018): The indicator side of tree microhabitats: A multi-taxon approach based on bats, birds and saproxylic beetles. In: J Appl Ecol 55 (5), S. 2147-2159. DOI: 10.1111/1365-2664.13181.

Palahí, Marc; Pantsar, Mari; Costanza, Robert; Kubiszewski, Ida; Potočnik, Janez; Stuchtey, Martin et al. (2020): Investing in Nature as the true engine of our economy. A 10-point Action Plan for a Circular Bioeconomy of Wellbeing. Knowledge to Action 02. European Forest Institute.

Paschke, Marian (2018): Vertragsnaturschutzentgelt im Privatwald. In: AFZ Der Wald (21), S. 3435. 
Petz, Maximilian von (2005): Umsetzungsmöglichkeiten des Vertragsnaturschutzes in der Forstwirtschaft. Dissertation.

Polley, Heino; Hennig, Petra (2015): Waldeigentum im Spiegel der Bundeswaldinventur. In: AFZ Der Wald, zuletzt geprüft am 09.12.2020.

Putensen, Alina (2018): Fallstudie über alternative Konzepte von Habitatbaumausweisungen. Masterarbeit. Georg-August-Universität Göttingen, Göttingen. Abteilung Forstökonomie, zuletzt geprüft am 18.11.2020.

Rat der Europäischen Gemeinschaften (1992): Richtlinie 92/43/EWG des Rates vom 21. Mai 1992 zur Erhaltung der natürlichen Lebensräume sowie der wildlebenden Tiere und Pflanzen. FFH-Richtlinie, zuletzt geprüft am 01.12.2020.

Rat der Europäischen Union (2003): Verordnung 1782/2003 des Rates mit gemeinsamen Regeln für Direktzahlungen im Rahmen der Gemeinsamen Agrarpolitik und mit bestimmten Stützungsregelungen für Inhaber landwirtschaftlicher Betriebe. EG VO 1872/2003.

Ring, Irene (Hg.) (2013): Der Nutzen von Ökonomie und Ökosystemleistungen für die Naturschutzpraxis. Dritte Veranstaltung der Workshop-Reihe des Bundesamtes für Naturschutz. Unter Mitarbeit von Imma Schniewind. Deutschland; Workshopreihe. Bonn: Bundesamt für Naturschutz (BfN-Skripten, 334). Online verfügbar unter http://www.bfn.de/fileadmin/MDB/documents/service/skript334.pdf.

Rosenkranz, Lydia; Wippel, Bernd; Seintsch, Björn (2012): FFH-Impact: Teil 1: Umsetzung der FFH-Richtlinie im Wald in den Bundesländern. Arbeitsbericht. Institut für Ökonomie der Forst- und Holzwirtschaft. Hamburg.

Sachverständigenrat für Umweltfragen; Wissenschaftlicher Beirat für Waldpolitik (Hg.) (2017): Für eine bessere Finanzierung des Naturschutzes in Europa nach 2020. Stellungnahme. Berlin.

Sagl, Wolfgang (1995): Bewertung in Forstbetrieben. Berlin: Blackwell-Wiss.-Verl. (Pareys Studientexte, 80).

Schnell, Martin; Schulz, Christian; Kolbe, Harald; Dunger, Christine (Hg.) (2013): Der Patient am Lebensende. Wiesbaden: Springer Fachmedien Wiesbaden.

Seintsch, Björn; Franz, Kristin; Meyer, Peter; Möhring, Bernhard (2018): Das WaVerNa- Forschungsprojekt im Überblick. In: AFZ Der Wald (21), S. 10-12.

Seintsch, Björn; Rosenkranz, Lydia; Englert, Hermann; Dieter, Matthias; Wippel, Bernd; Becker, Gero et al. (2012): FFH-Impact: Teil 2: Auswirkungen von FFH-Maßnahmenplanungen auf Forstbetriebe. Hamburg, zuletzt geprüft am 25.11.2020.

Seintsch, Björn; Selzer, Anne M.; Dieter, Matthias; Möhring, Bernhard; von Blomberg, Georg Moritz Freiherr; Demant, Laura et al. (2019): Gemeinsamer Schlussbericht zum Verbundvorhaben Vertragsnaturschutz im Wald- Analyse der waldökologischen, ökonomischen und rechtlichen Optionen. WaVerNa-Projekt FK 22007115. Hg. v. Georg-August-Universität Göttingen, Nordwestdeutsche Forstliche Versuchsanstalt, Thünen-Institut und Universität Hamburg.

Selzer, Anne Mira (2018): Übersicht über die Instrumente der staatlichen Nachfrager. Status quo der Umsetzung von Naturschutz im Wald gegen Entgelt in Deutschland. Hg. v. ThünenInstitut. Braunschweig (Thünen Working Paper, 83).

Sotirov, Metodi (Hg.) (2017): Natura 2000 and forests. Assessing the state of implementation and effectiveness. European Forest Institute (What science can tell us, 7). 
Suda, Michael; Pukall, Klaus (2014): Multifunktionale Forstwirtschaft zwischen Inklusion und Extinktion (Essay). In: Schweizerische Zeitschrift fur Forstwesen 165 (11), S. 333-338. DOI: 10.3188/szf.2014.0333.

Thoroe, Carsten (2009): Wald im Wandel - gesamtgesellschaftliche Herausforderungen. In: Landbauforschung - Applied Agricultural and Forestry Research (327), S. 5-9, zuletzt geprüft am 09.12.2020.

Thüringer Ministerium für Infrastruktur und Landwirtschaft (2019): Thüringer Richtlinie zur Förderung forstwirtschaftlicher Maßnahmen. In: Thüringer Staatsanzeiger Nr. 39/2019, S. 1019-1042, zuletzt geprüft am 19.11.2020.

Volckens, Frederik (2014): GAP-Reform: Betriebswirtschaftliche Fragen \& Antworten. BB Göttingen $\mathrm{GmbH}$. Leinefelde-Worbis, 10.02.2014.

Warnecke, Rüdiger; Hertell, Felix-Michael von; Scheffler, Winfried; Amelung, Cord; Friedrichs, Jan-Christoph; Riedel, Joachim et al. (2019): Betriebsvergleich 2017/2018. Hg. v. BB Göttingen $\mathrm{GmbH}$. Göttingen.

Wätzold, Frank; Wissel, Silvia (2016): German Impact Mitigation Regulation in Hessen. Hg. v. OECD (Biodiversity Offsets: Effective Design and Implementation), zuletzt geprüft am 25.11.2020.

Weber, Anja (2015): Implementing EU co-financed agri-environmental schemes: Effects on administrative transaction costs in a regional grassland extensification scheme. In: Land Use Policy 42, S. 183-193. DOI: 10.1016/j.landusepol.2014.07.019.

Windstoßer, Carola (2008): Vertragsnaturschutz. Ein Verwaltungsinstrument mit ungewisser Zukunft? 1. Auflage. Berlin: Duncker \& Humblot GmbH (Tübinger Schriften zum Staats- und Verwaltungsrecht). Online verfügbar unter https://www.wisonet.de/document/DUHU_9783428528349258.

Wissenschaftlicher Beirat für Waldpolitik; Wissenschaftlicher Beirat für Biodiversität und Genetische Ressourcen (Hg.) (2020): Effizienter Waldnaturschutz in Deutschland. Stellungnahme. Berlin. 
9. Anhang

9.1.1. Fragebogen WaVerNa Fallbeispielsanalysen

\begin{tabular}{|c|c|c|}
\hline \multicolumn{3}{|c|}{ ÜBERWIEGEND VORERHEBUNG } \\
\hline \multirow[t]{7}{*}{ A. } & \multicolumn{2}{|l|}{ Kontaktdaten } \\
\hline & Name, Vorname & \\
\hline & Straße, Hausnr. & \\
\hline & Postleitzahl & \\
\hline & Email & \\
\hline & Telefon & \\
\hline & Funktion des Antwortenden & $\begin{array}{l}\square \text { Eigentümer } \\
\square \text { Betriebsleiter } \\
\square \text { Vorstand einer Forstbetriebsgemeinschaft, einer } \\
\text { Forstgenossenschaft oder einer Realgemeinde } \\
\square \text { Betreuungsförster } \\
\square \text { Externer Dienstleister } \\
\square \text { Sonstige }\end{array}$ \\
\hline & \multicolumn{2}{|l|}{ Weiterer Ansprechpartner im Betrieb } \\
\hline & Name, Vorname & \\
\hline & Email & \\
\hline & Telefon & \\
\hline & Funktion & \\
\hline B. & Betriebsdaten & \\
\hline & Eigentumsart & $\begin{array}{l}\square \text { Privatwald Einzelunternehmen }->\text { Einzeleigentum } \\
\square \quad \text { Privatwald Genossenschaftswald }->\text { Gemein- } \\
\text { schaftseigentum (einschließlich rechtlich gleichge- } \\
\text { stellter Gemeinschaftswälder) } \\
\square \text { Stiftungswald } \\
\square \text { Kommunalwald } \\
\square \text { Kirchenwald } \\
\square \text { Landeswald } \\
\square \text { Bundeswald }\end{array}$ \\
\hline & Betriebsteile bei Privatwald & $\begin{array}{l}\square \text { Forstwirtschaft } \\
\square \text { Landwirtschaft } \\
\square \\
\text { dere: }\end{array}$ \\
\hline & $\begin{array}{l}\text { Größe der gesamten Betriebsfläche bei } \\
\text { land- und forstwirtschaftlichem Betrieb } \\
\text { (in ha) }\end{array}$ & \\
\hline & $\begin{array}{l}\text { Größe der forstlichen Betriebsfläche (in } \\
\text { ha) }\end{array}$ & \\
\hline & Verteilung der Baumartengruppen & $\begin{array}{l}\text { — Eiche } \\
\text { — Buche (inkl. sonst. Laubholz) } \\
\text { — } \% \text { Fichte (inkl. Tanne, Douglasie) } \\
\text { \% Kiefer (inkl. Lärche \& sonst. Nadelholz) }\end{array}$ \\
\hline
\end{tabular}




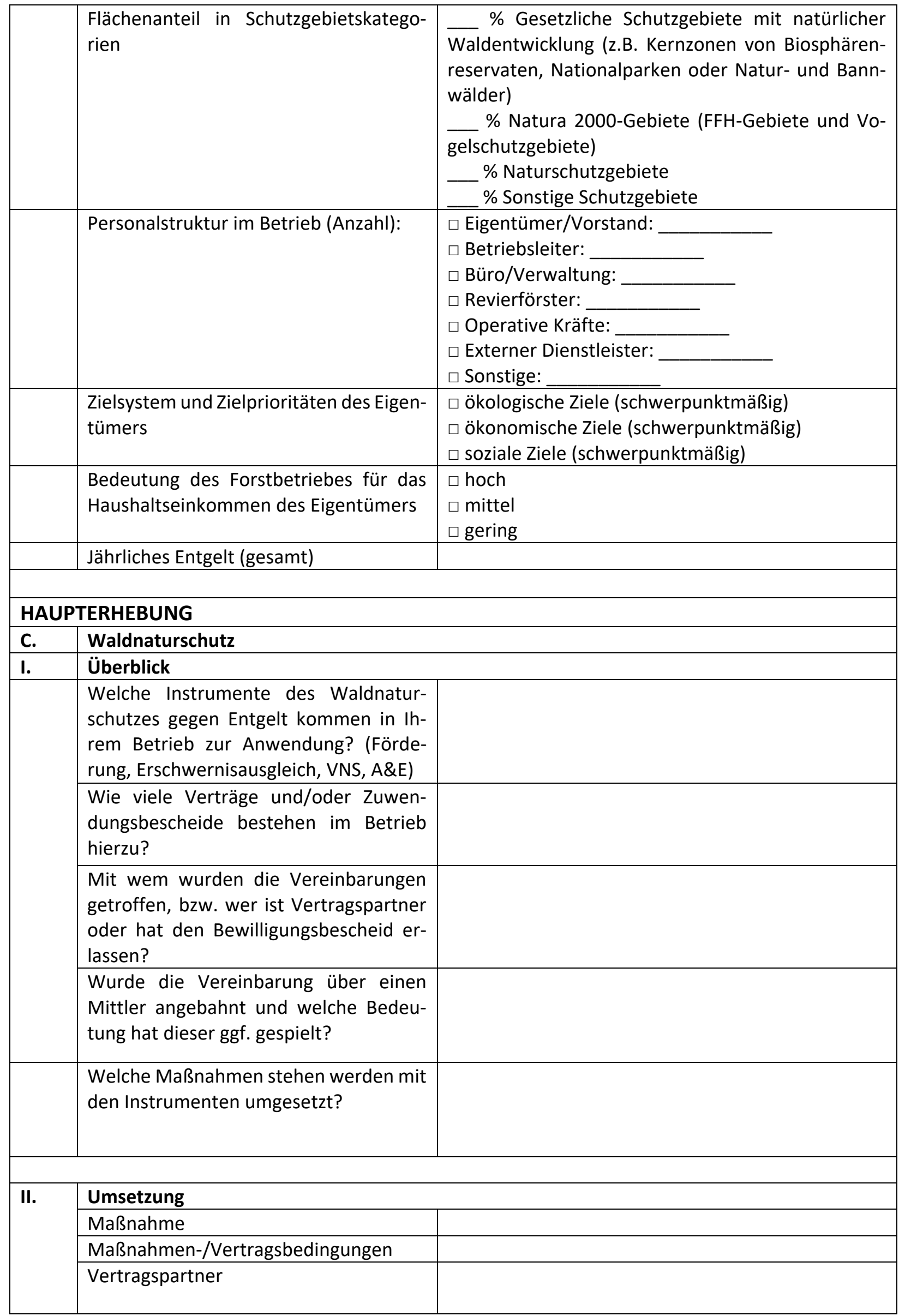




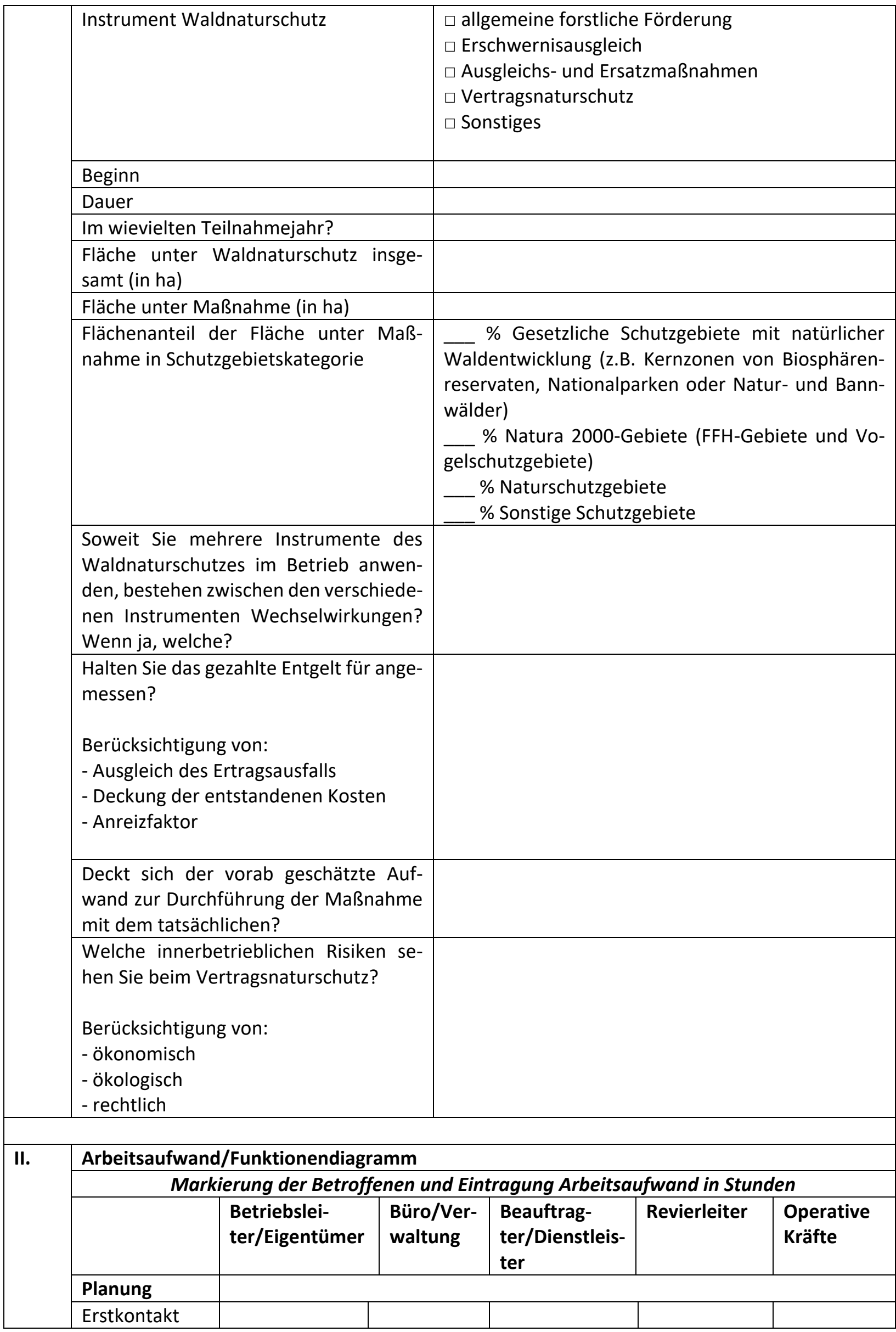




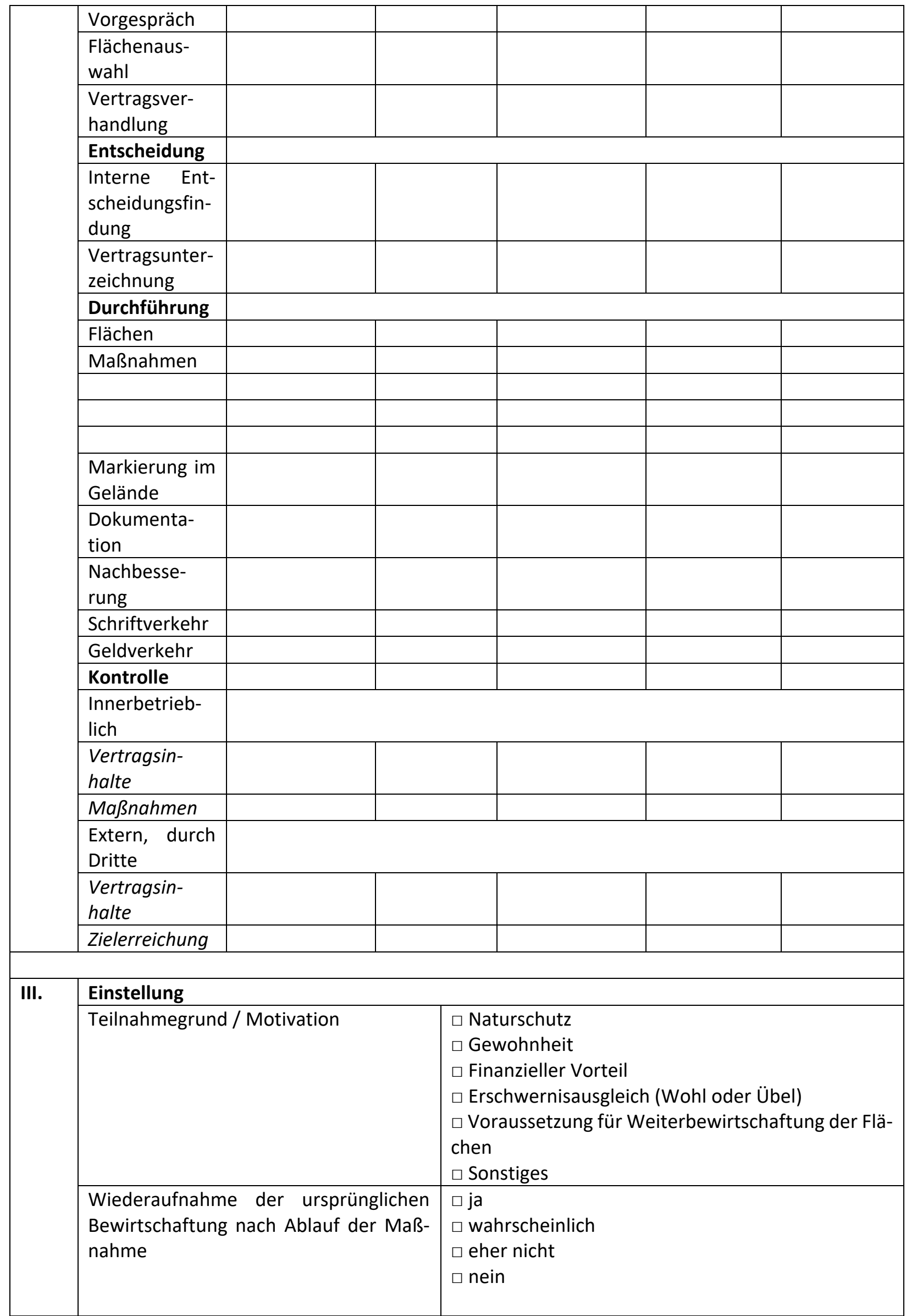




\begin{tabular}{|c|c|c|}
\hline & Zertifizierung & $\begin{array}{l}\square \text { Ja, PEFC } \\
\square \text { Ja, FSC } \\
\square \text { Ja, Naturland } \\
\square \text { Nein }\end{array}$ \\
\hline & Beginn der Zertifizierung & \\
\hline IV. & Erfahrung & \\
\hline & $\begin{array}{l}\text { Aktuell Durchführung von Naturschutz- } \\
\text { maßnahmen gegen Entgelt in anderem } \\
\text { Betriebszweig? (bei Mischbetrieb) }\end{array}$ & $\begin{array}{l}\square \text { ja, } \\
\text { und } \\
\text { zwar: }\end{array}$ \\
\hline & & $\begin{array}{l}\text { seit: } \\
\text { anein }\end{array}$ \\
\hline & $\begin{array}{l}\text { In der Vergangenheit Durchführung von } \\
\text { Naturschutzmaßnahmen gegen Entgelt } \\
\text { in anderem Betriebszweig? (bei Misch- } \\
\text { betrieb) }\end{array}$ & $\begin{array}{l}\square \text { ja, } \\
\text { und } \\
\text { zwar: }\end{array}$ \\
\hline & & $\begin{array}{l}\text { von } \_ \text {bis } \\
\text { nein }\end{array}$ \\
\hline & $\begin{array}{l}\text { In der Vergangenheit Durchführung von } \\
\text { Maßnahmen des Waldnaturschutzes ge- } \\
\text { gen Entgelt auf forstlicher Betriebsflä- }\end{array}$ & $\begin{array}{l}\square \text { ja, } \\
\text { und } \\
\text { zwar: }\end{array}$ \\
\hline & & $\begin{array}{l}\text { von__ bis } \_ \text {__ } \\
\text { nein }\end{array}$ \\
\hline V. & Fachlicher Hintergrund & \\
\hline & Alter & \\
\hline & Höchster Schulabschluss & $\begin{array}{l}\square \text { kein Abschluss } \\
\square \text { Volks- bzw. Hauptschule } \\
\square \text { Realschule } \\
\square \text { Fachoberschule/ Abitur }\end{array}$ \\
\hline & $\begin{array}{l}\text { Höchster fachspezifischer Bildungsab- } \\
\text { schluss }\end{array}$ & $\begin{array}{l}\square \text { Keine Ausbildung } \\
\square \text { Lehre } \\
\square \text { Meisterprüfung (Bspw.:Forstwirtschaftmeister) } \\
\square \text { Studium }\end{array}$ \\
\hline & $\begin{array}{l}\text { Höchster Bildungsabschluss in anderen } \\
\text { Berufsfeldern }\end{array}$ & $\begin{array}{l}\square \text { Keine Ausbildung } \\
\square \text { Lehre } \\
\square \text { Meisterprüfung/ Techniker } \\
\square \text { Studium }\end{array}$ \\
\hline & $\begin{array}{l}\text { Freiwillige Mitgliedschaft in berufsstän- } \\
\text { diger Vereinigung /Verband }\end{array}$ & $\begin{array}{l}\square \text { ja, } \\
\text { und } \\
\text { zwar: }\end{array}$ \\
\hline
\end{tabular}




\begin{tabular}{|c|c|c|}
\hline & & $\square$ nein \\
\hline & Regelmäßiger Bezug von Fachpresse & $\begin{array}{l}\square \text { ja, } \\
\text { und } \\
\text { zwar: }\end{array}$ \\
\hline & & $\square$ nein \\
\hline & $\begin{array}{l}\text { Regelmäßige Teilnahme an Weiterbil- } \\
\text { dungs-und Fortbildungsangeboten }\end{array}$ & $\begin{array}{l}\square \text { ja, } \\
\square \text { nein }\end{array}$ \\
\hline VI. & Probleme & \\
\hline & $\begin{array}{l}\text { Welche Probleme sind während, vor } \\
\text { oder nach der Vertragslaufzeit aufgetre- } \\
\text { ten } \\
\text { Berücksichtigung von: } \\
\text { - Maßnahme } \\
\text { - Vertragspartner } \\
\text { - Sonstige }\end{array}$ & \\
\hline & $\begin{array}{l}\text { Welche grundsätzlichen Probleme se- } \\
\text { hen Sie persönlich beim VNS? }\end{array}$ & \\
\hline & $\begin{array}{l}\text { Welche grundsätzlichen Chancen sehen } \\
\text { Sie persönlich beim VNS? }\end{array}$ & \\
\hline & $\begin{array}{l}\text { In welchem Bereich bestand die größten } \\
\text { Zielkonflikte zwischen dem Anbieter, } \\
\text { Nachfrager und Vermittler }\end{array}$ & \\
\hline VII. & Rechtliches & \\
\hline & $\begin{array}{l}\text { Sind Sie mit den in Ihrem Bundesland zur } \\
\text { Verfügung stehenden Instrumenten zu- } \\
\text { frieden? Welche Elemente bewerten sie } \\
\text { positiv und wo sehen Sie Nachbesse- } \\
\text { rungsbedarf? }\end{array}$ & \\
\hline & $\begin{array}{l}\text { Welche Erfahrungen bewegen Sie dazu } \\
\text { sich künftig mehr/weniger zu engagie- } \\
\text { ren? }\end{array}$ & \\
\hline & $\begin{array}{l}\text { Welche konkreten Inhalte von Natur- } \\
\text { schutzvereinbarungen gegen Entgelt } \\
\text { halten Sie für besonders relevant bzw. } \\
\text { würden Sie dazu animieren, einen Ver- } \\
\text { trag abzuschließen? }\end{array}$ & \\
\hline & $\begin{array}{l}\text { Welche Empfehlungen würden Sie auf } \\
\text { Grundlage Ihrer Erfahrungen für die }\end{array}$ & \\
\hline
\end{tabular}




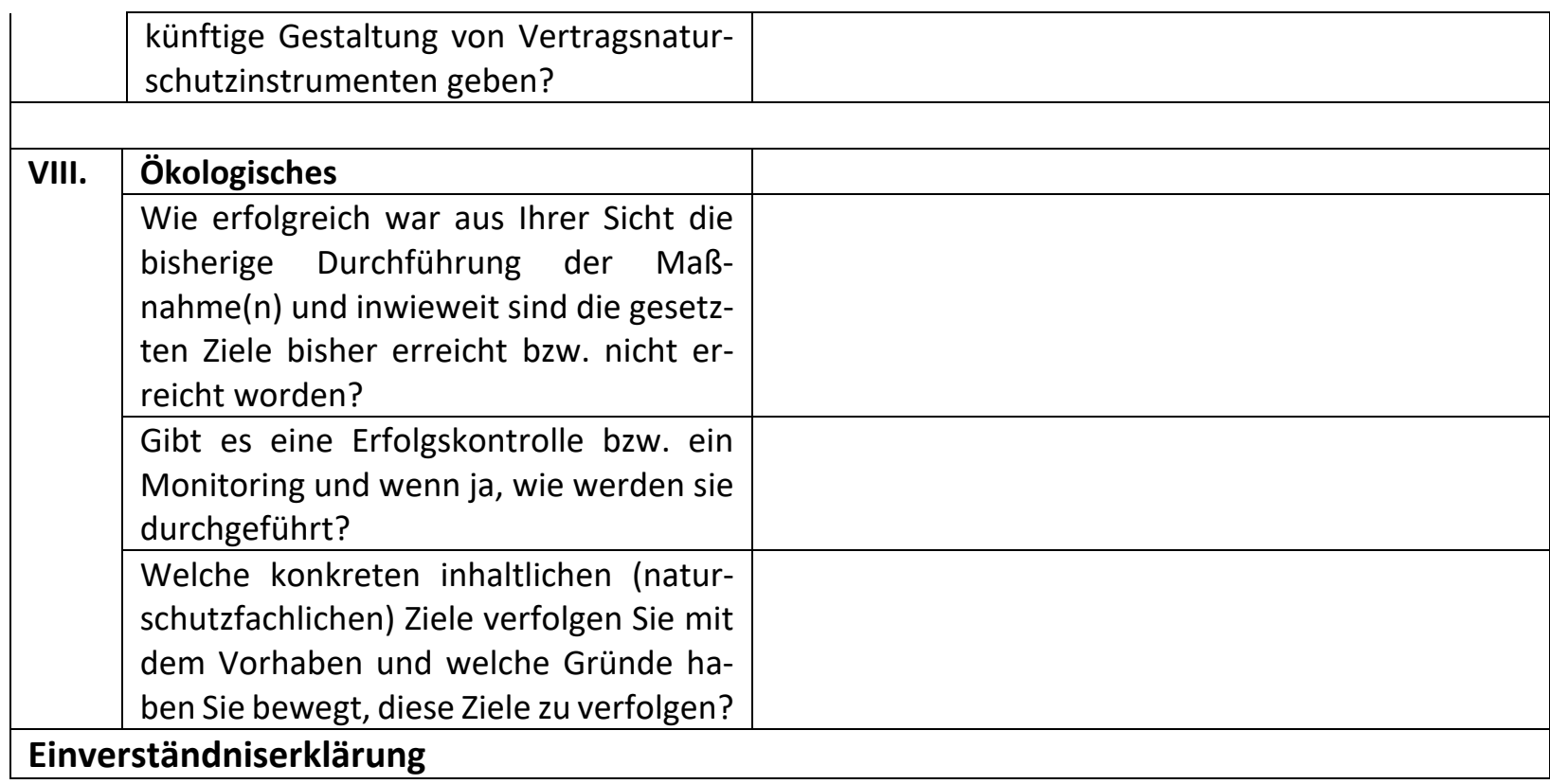




\subsection{2. Ökologischer Bewertungskatalog Habitatbäume}

Tabelle 20: Bewertungskatalog der Habitatmerkmale von Einzelbäumen, abgewandelt und ergänzt nach Meier (2009). Die Punktzahl eines Habitatbaums entspricht der Summe der Punkte seiner Merkmale, Quelle: Putensen 2018

\begin{tabular}{|c|c|c|}
\hline Merkmal & ggf. Beschreibung & Punkte \\
\hline \multirow{3}{*}{$\begin{array}{l}\text { Äste } \\
\text { (D: Durchmesser) } \\
\text { (L: Länge) }\end{array}$} & tot, $\mathrm{D}>10 \mathrm{~cm}, \mathrm{~L}>50 \mathrm{~cm}$ & 13,25 \\
\hline & lebend oder tot, $D>10, L<50$ (Astbruch) & 10 \\
\hline & lebend abgebrochen, $D>10, L>50$ & 10 \\
\hline \multirow[t]{2}{*}{ Höhlen } & In $<4$ m Höhe & 10 \\
\hline & In $>4$ m Höhe & 11,5 \\
\hline Rindenschürfung & (alle) & 9,25 \\
\hline Rindentasche & (alle) & 11,5 \\
\hline Risse und Spalten & (alle) & 11,5 \\
\hline Moos & $>25 \%$ & 9 \\
\hline Flechte & $>25 \%$ & 10 \\
\hline Efeu & $>25 \%$ & 6 \\
\hline \multicolumn{2}{|c|}{ Konsolenpilzfruchtkörper } & 10 \\
\hline \multicolumn{2}{|c|}{ Hohlraum Stammfuß oder Wurzelanlauf } & 9 \\
\hline \multicolumn{2}{|l|}{ Horst } & 6 \\
\hline \multicolumn{2}{|c|}{ Hohler Baum oben geschlossen } & 13 \\
\hline \multicolumn{2}{|c|}{ Hohler Baum oben offen } & 14 \\
\hline \multicolumn{2}{|l|}{ Zwiesel } & 6 \\
\hline \multicolumn{2}{|l|}{ Stockausschlag } & 4 \\
\hline \multicolumn{2}{|l|}{ Saftfluss, Schleimfluss } & 10 \\
\hline \multirow[t]{2}{*}{ Dendrotelm } & unterhalb von $3 \mathrm{~m}$ & 9 \\
\hline & oberhalb von $3 \mathrm{~m}$ & 12 \\
\hline \multicolumn{2}{|c|}{ Rindenschäden ohne frl. Holz } & 10 \\
\hline \multicolumn{2}{|c|}{ Mulm (in Höhlen, Spalten, Rindentaschen) } & 9 \\
\hline \multicolumn{2}{|l|}{ Krebs } & 7 \\
\hline \multicolumn{2}{|l|}{ Hexenbesen } & 6 \\
\hline \multicolumn{2}{|c|}{ Starkastigkeit / tiefe Äste } & 9 \\
\hline \multicolumn{2}{|l|}{ Kronenbruch } & 11 \\
\hline \multicolumn{2}{|c|}{ Pilzfruchtkörper (keine Konsolen, z.B. Goldfellschüppling) } & 10 \\
\hline \multicolumn{2}{|c|}{ Fäule (Pilzbefall an freiliegendem Holz ohne Fruchtkörper) } & 5 \\
\hline \multicolumn{2}{|l|}{ Schrägstellung } & 5 \\
\hline \multirow[t]{2}{*}{ Baumart } & Buche & 1 \\
\hline & Eiche & 4 \\
\hline \multirow[t]{5}{*}{ BHD } & $<40 \mathrm{~cm}$ & 0 \\
\hline & $40-<60$ & 1 \\
\hline & $60-<80$ & 2 \\
\hline & $80-<100$ & 3 \\
\hline & $\geq 100$ & 4 \\
\hline
\end{tabular}




\subsubsection{Fragebogen BB-Forstbetriebe}

\section{Befragung zum Naturschutz gegen Entgelt im Wald \& in der Landwirtschaft}

Sehr geehrte Damen und Herren, liebe Teilnehmer des BB-Forstbetriebsvergleichs

im vergangenen Jahr haben wir im Vorwort des Forstbetriebsvergleichs bereits angekündigt und dieses Jahr beim Forstseminartag vorgestellt, dass wir Sie bezüglich Ihrer Erfahrungen mit Naturschutz gegen Entgelt im Wald gerne befragen möchten.

Von der Gesellschaft werden steigende Naturschutzansprüche an den Wald gestellt, auch an den Privatwald. Bisher wurden im Wald Ansprüche überwiegend durch hoheitliche Instrumente auf ordnungsrechtlicher Basis durchgesetzt. Nach Bundesnaturschutzgesetz und im Einklang mit Forderungen aus Verbänden und der Politik soll jedoch bei Naturschutzvorhaben vorrangig geprüft werden, ob der Schutzzweck nicht auch mit vertraglichen Vereinbarungen erreicht werden kann. Dieser Vertragsnaturschutz ist in der Landwirtschaft als etabliertes Erfolgsmodell bekannt, im Wald bislang jedoch nur rudimentär umgesetzt.

In den vergangenen zwei Jahren hat unser gemeinsames Forschungsvorhaben (WaVerNa-Projekt) intensiv die vorhandenen Ansätze untersucht, mittels einer offenen Onlinebefragung den Status quo erhoben und in ausgewählten Fallstudien die Potentiale und Hemmnisse analysiert. Für meine eigene Arbeit, die Untersuchung der Auswirkungen von Naturschutz gegen Entgelt im Wald auf betriebswirtschaftliche und organisatorische Aspekte in Forstbetrieben, benötige ich jedoch weitere Informationen und Ihre Einschätzungen als Experten auf dem Gebiet der erwerbsorientierten Waldbewirtschaftung.

Daher möchte ich Sie bitten, den anliegenden Fragebogen vorzubereiten, um ihn mit mir zusammen in einem anschließenden Telefongespräch zu erarbeiten. Dazu würde ich Sie gerne innerhalb der nächsten vier Wochen kontaktieren. Wenn Sie nicht an der Befragung teilnehmen möchten, bitte ich Sie, mir dies kurz per Email oder Telefon mitzuteilen.

Mit freundlichem Gruß und herzlichem Dank vorab

\section{Betriebsumfrage Naturschutz gegen Entgelt}

Betrieb:

Funktion des Antwortenden:

Seit wann in Funktion:

Wenn Sie neben Ihrem Forstbetrieb keine Landwirtschaft betreiben, lassen Sie die Fragen zur Landwirtschaft bitte aus.

\section{Allgemein:}

1. Wie groß ist die forstliche Fläche Ihres Betriebs?

2. Wie groß ist die landwirtschaftliche Fläche Ihres Betriebs? 
3. Welche Bedeutung haben folgende Ziele in Ihrem Betrieb für Sie?

\begin{tabular}{|c|c|c|c|c|c|c|c|c|c|c|c|c|}
\hline \multirow[t]{2}{*}{ Ziele: } & \multicolumn{6}{|c|}{ Forst } & \multicolumn{6}{|c|}{ Landwirtschaft } \\
\hline & 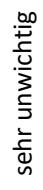 & 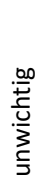 & 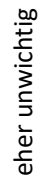 & 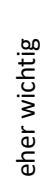 & 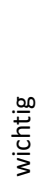 & 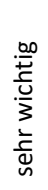 & 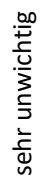 & 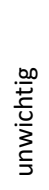 & 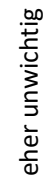 & 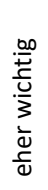 & 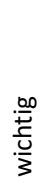 & 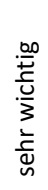 \\
\hline $\begin{array}{l}\text { Rentabili- } \\
\text { tät/Ertrag }\end{array}$ & & & & & & & & & & & & \\
\hline $\begin{array}{l}\text { Liquidi- } \\
\text { tätssiche- } \\
\text { rung }\end{array}$ & & & & & & & & & & & & \\
\hline $\begin{array}{l}\text { Vermö- } \\
\text { genser- } \\
\text { halt/Wert } \\
\text { nachhal- } \\
\text { tigkeit }\end{array}$ & & & & & & & & & & & & \\
\hline $\begin{array}{l}\text { Schön- } \\
\text { heit/Land } \\
\text { schaftsge- } \\
\text { staltung }\end{array}$ & & & & & & & & & & & & \\
\hline Jagd & & & & & & & & & & & & \\
\hline $\begin{array}{l}\text { Natur-, } \\
\text { Umwelt- } \\
\text { schutz }\end{array}$ & & & & & & & & & & & & \\
\hline Tradition & & & & & & & & & & & & \\
\hline Sonstiges: & & & & & & & & & & & & \\
\hline
\end{tabular}

4. Wie hoch ist der Anteil Natura 2000-Flächen in Ihrem Forstbetrieb? Mit welchem Schutzstatus sind diese versehen?

5. Haben Sie Naturschutzmaßnahmen im Wald gegen Entgelt durchgeführt? Wenn Nein, warum nicht?

6. Welche Naturschutzmaßnahme gegen Entgelt haben Sie im Wald in den letzten 20 Jahren durchgeführt? Waren diese an die Natura 2000-Kulisse gebunden? Welchen Anteil des Betriebsertrages haben sie ausgemacht?

7. Haben Sie in der Landwirtschaft an Agrarumweltmaßnahmen oder anderem Naturschutz gegen Entgelt teilgenommen? In welchem Zeitraum? Welchen Anteil des Betriebsertrages haben sie ausgemacht?

8. Wie wichtig sind folgende Zielsetzungen für die Teilnahme an Naturschutz gegen Entgelt? 


\begin{tabular}{|c|c|c|c|c|c|c|c|c|c|c|c|c|}
\hline & \multicolumn{6}{|c|}{ Forst } & \multicolumn{6}{|c|}{ Landwirtschaft } \\
\hline & 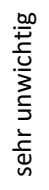 & 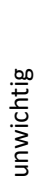 & 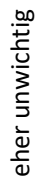 & 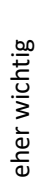 & & 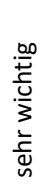 & 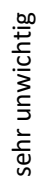 & 紫 & 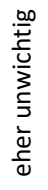 & 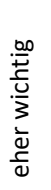 & 站 & 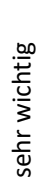 \\
\hline $\begin{array}{l}\text { Land- } \\
\text { schaftsge- } \\
\text { staltung }\end{array}$ & & & & & & & & & & & & \\
\hline $\begin{array}{l}\text { Natur-, } \\
\text { Umwelt- } \\
\text { schutz }\end{array}$ & & & & & & & & & & & & \\
\hline Tradition & & & & & & & & & & & & \\
\hline $\begin{array}{l}\text { Gesell- } \\
\text { schaftliche } \\
\text { Akzeptanz }\end{array}$ & & & & & & & & & & & & \\
\hline $\begin{array}{l}\text { Rentabili- } \\
\text { tät }\end{array}$ & & & & & & & & & & & & \\
\hline $\begin{array}{l}\text { Diversifi- } \\
\text { zierung }\end{array}$ & & & & & & & & & & & & \\
\hline $\begin{array}{l}\text { Vermei- } \\
\text { dung Ord- } \\
\text { nungsrecht }\end{array}$ & & & & & & & & & & & & \\
\hline
\end{tabular}

9. Wie beurteilen Sie folgende Merkmale Ihrer Naturschutzmaßnahme gegen Entgelt im Wald und Ihrer Agrarumweltmaßnahmen?

\begin{tabular}{|c|c|c|c|c|c|c|c|c|c|c|c|c|}
\hline & \multicolumn{6}{|c|}{ Natursch.-Maß. im Wald } & \multicolumn{6}{|c|}{ Agrarumweltmaßnahmen } \\
\hline & 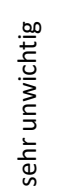 & 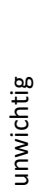 & 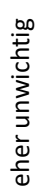 & 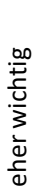 & & 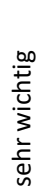 & 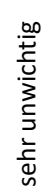 & .0.0. & 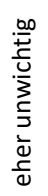 & 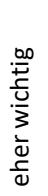 & & 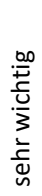 \\
\hline $\begin{array}{l}\text { Forstbe- } \\
\text { triebliche } \\
\text { Angepasst- } \\
\text { heit }\end{array}$ & & & & & & & & & & & & \\
\hline $\begin{array}{l}\text { Angemes- } \\
\text { senheit des } \\
\text { Entgelts }\end{array}$ & & & & & & & & & & & & \\
\hline $\begin{array}{l}\text { Kontinuität } \\
\text { der Finan- } \\
\text { zierung }\end{array}$ & & & & & & & & & & & & \\
\hline $\begin{array}{l}\text { Rechtsun- } \\
\text { sicherheit }\end{array}$ & & & & & & & & & & & & \\
\hline
\end{tabular}


10. Wo gab es in Ihrem Betrieb Probleme bei der Umsetzung von Naturschutz gegen Entgelt?
a) Im Wald:
b) In der Landwirtschaft:

11. Wo sehen Sie Stärken von Naturschutz gegen Entgelt?
a) Im Wald:
b) In der Landwirtschaft:

12. Welche Möglichkeit sehen Sie folgende Punkte des Naturschutzes im Wald zu verbessern?
a. Forstbetriebliche Angepasstheit
b. Finanzielle Angemessenheit
c. Kontinuität der Finanzierung
d. Rechtsunsicherheit

13. Ist aus Ihrer Sicht eine Vermarktung von Einzelobjekten wie Bäumen oder ein Flächenbezug vorzuziehen?

14. Was sind aus Ihrer Sicht besondere „Best practice“-Beispiele beim Naturschutz gegen Entgelt im Wald? Was wäre aus betrieblicher Sicht eine besonders attraktive Lösung für Naturschutz gegen Entgelt?

15. Was sind für Sie abschließende Erkenntnisse aus den Agrarumwelt- und Klimamaßnahmen aus der Landwirtschaft für Naturschutz gegen Entgelt im Wald? 
9.1.4. Ergebnisse Bewertungskonzept für Einzelbäume
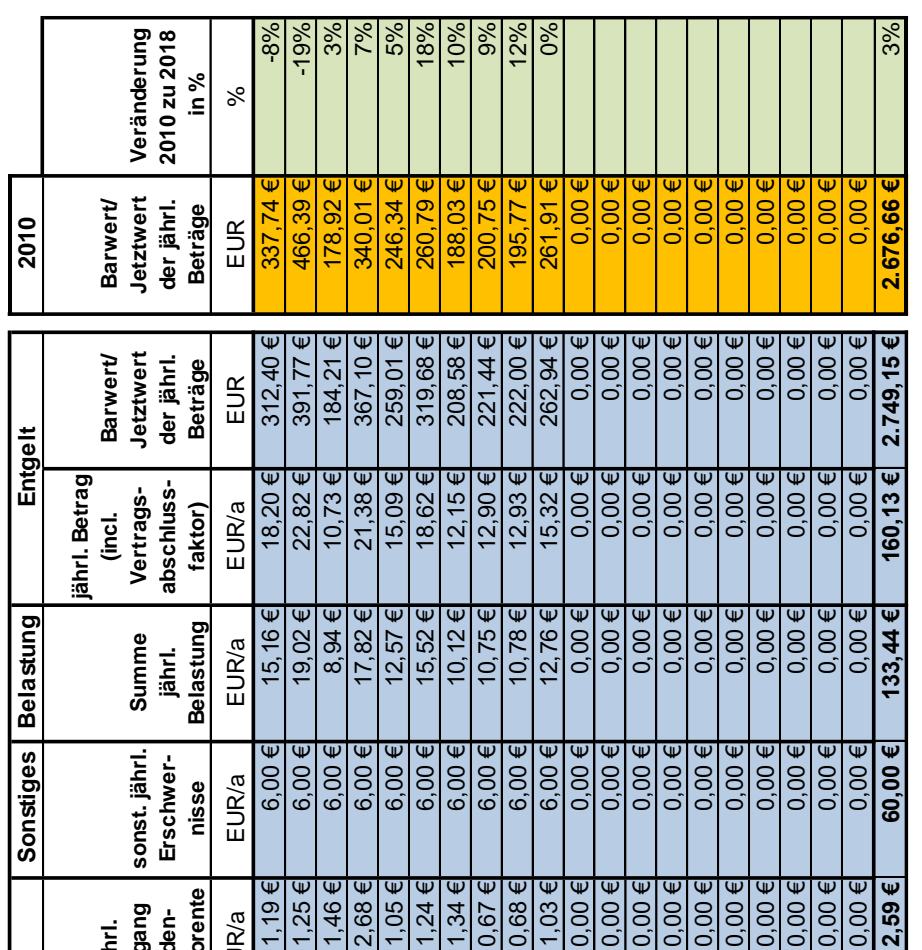

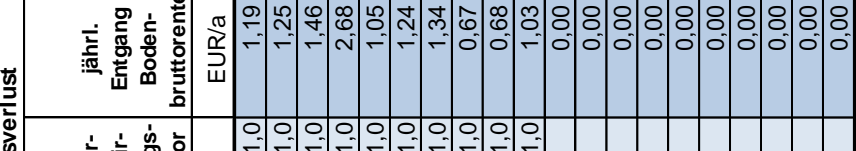

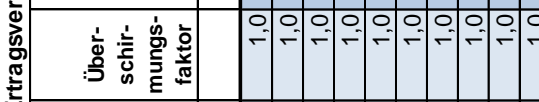

造它

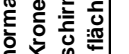
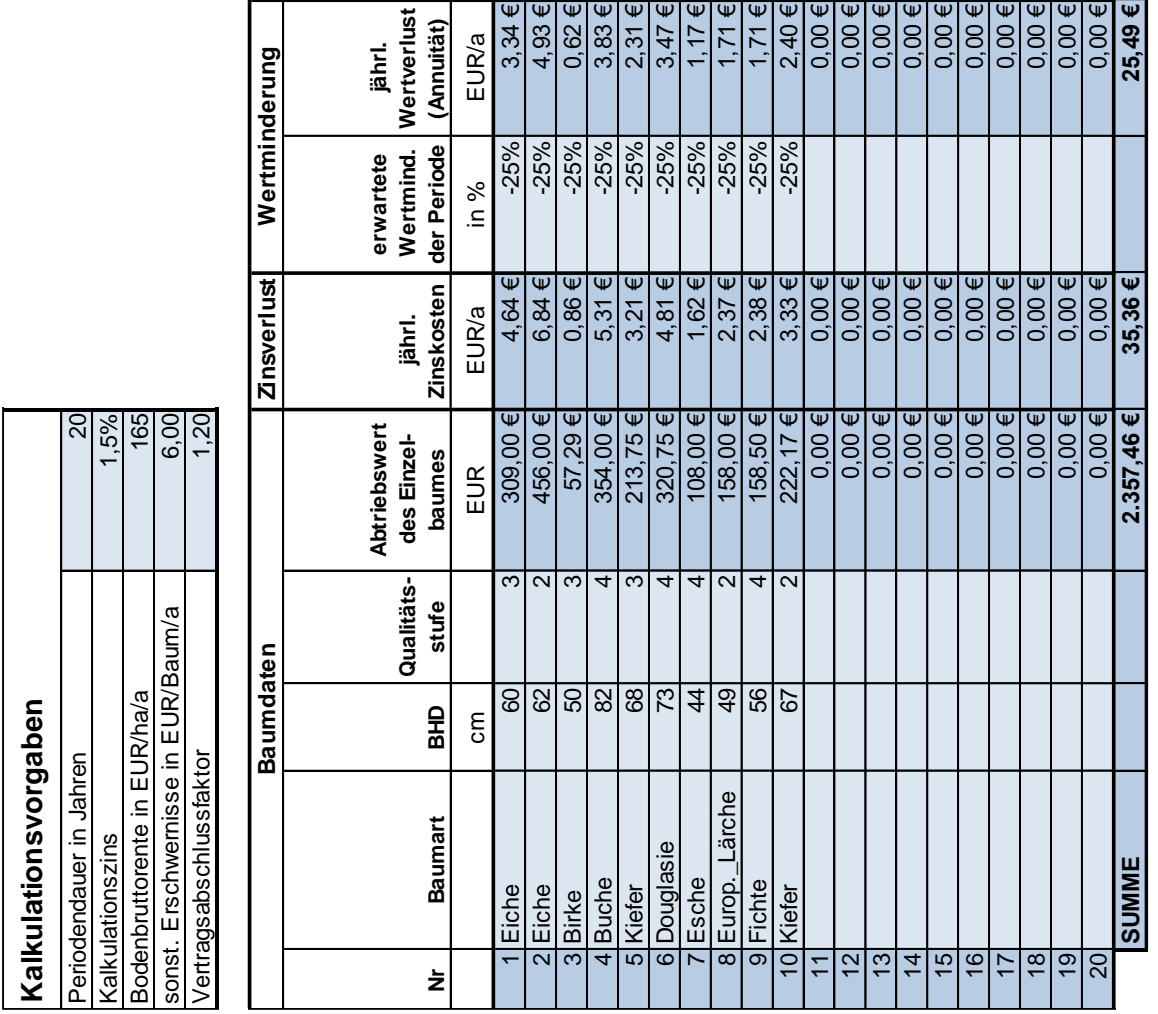
9.1.5. Eingangsdaten der verglichenen Kalkulationstools für forstliche Nutzungsbeschränkungen

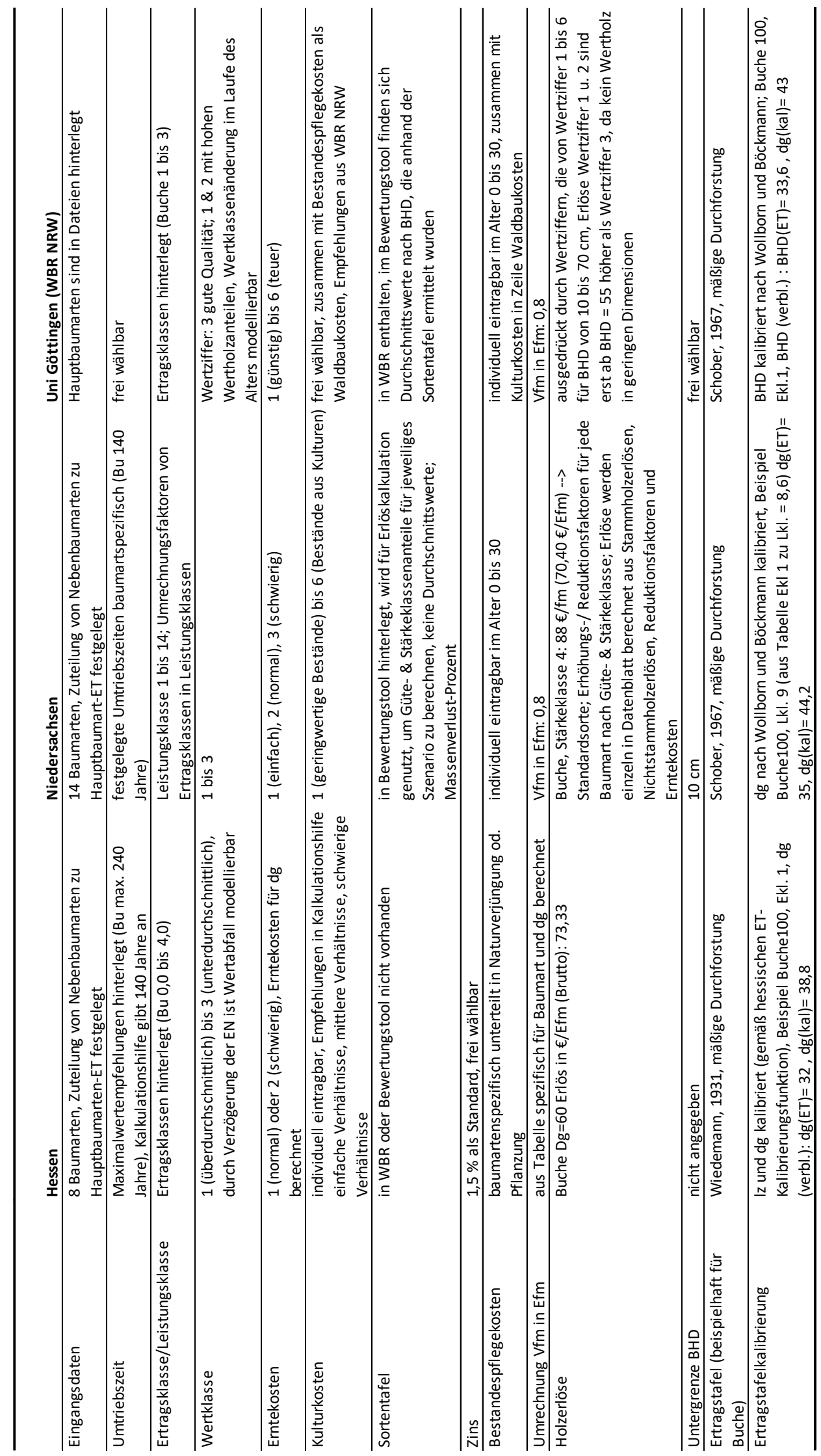

